Supporting Information (SI)

\title{
Switching Dual Catalysis without Molecular Switch: Using A Multi- Component Information System for Reversible Reconfiguration of Catalytic Machinery
}

\author{
Abir Goswami, ${ }^{\mathrm{a}}$ Thomas Paululat, ${ }^{\mathrm{b}}$ and Michael Schmittel ${ }^{\mathrm{a}, *}$ \\ ${ }^{a}$ Organische Chemie I, ${ }^{b}$ Organische Chemie II. Center of Micro- and Nanochemistry and \\ Engineering, University of Siegen, Adolf-Reichwein-Str. 2, D-57068 Siegen, Germany. \\ E-mail: schmittel@chemie.uni-siegen.de
}

Fax: (+49) 271-740-14357

\section{Table of Contents}

1. Synthesis.

2. Synthesis and characterization of complexes....

S7-S16

3. Model study.

S17

4. NMR spectra.

5. DOSY NMR spectra.

6. Variable temperature study and determination of kinetic parameters

7. Catalytic experiments

8. ESI-MS spectra

9. UV-vis data. 


\section{Synthesis}

\section{General Remarks}

All solvents were dried by distillation prior to use while commercial reagents $(\mathbf{1}, \mathbf{3}, \mathbf{6}, \mathbf{7}, \mathbf{A 2}, \mathbf{B 1}, \mathbf{1 4}$, hexacyclen) were used without any further purification. Bruker Avance (400 MHz) and Varian VNMR-S $600(600 \mathrm{MHz})$ spectrometers were used to measure ${ }^{1} \mathrm{H}$ and ${ }^{13} \mathrm{C}$ NMR spectra using a deuterated solvent as the lock and residual protiated solvent as internal reference $\left(\mathrm{CDCl}_{3}: \delta_{\mathrm{H}} 7.26\right.$ ppm, $\delta_{\mathrm{C}} 77.0$ ppm; $\mathrm{CD}_{2} \mathrm{Cl}_{2}: \delta_{\mathrm{H}} 5.32 \mathrm{ppm}, \delta_{\mathrm{C}} 53.8 \mathrm{ppm}, \mathrm{THF}-\mathrm{d}_{8}: \delta_{\mathrm{H}} 1.72 \mathrm{ppm}, 3.58 \mathrm{ppm}, \delta_{\mathrm{C}} 25.3$ ppm, $67.2 \mathrm{ppm})$. The following abbreviations were used to define NMR peak pattern: $\mathrm{s}=$ singlet, $\mathrm{d}$ $=$ doublet, $\mathrm{t}=$ triplet, $\mathrm{dd}=$ doublet of doublets, $\mathrm{ddd}=$ doublet of doublets of doublets, $\mathrm{td}=$ triplet of doublets, $\mathrm{br}=$ broad, $\mathrm{m}=$ multiplet. Coupling constant values are given in Hertz $(\mathrm{Hz})$ and, wherever possible, assignment of protons is provided. The numbering of different carbons in different molecular skeletons does not necessarily follow IUPAC nomenclature rules; it was exclusively implemented for assigning NMR signals. All electrospray ionization (ESI-MS) spectra were recorded on a Thermo-Quest LCQ deca and theoretical isotopic distributions of the mass signals were calculated using IsoPro 3.0 software. Melting points of compounds were measured on a BÜCHI 510 instrument and are not corrected. Infrared spectra were recorded on a Varian 1000 FTIR instrument. Elemental analysis was performed using the EA-3000 CHNS analyzer. UV-vis spectra were recorded on a Cary Win 50 (298 K) spectrometer. Binding constants were determined through UV-vis titrations in combination with a 1:1 binding formula of two ligands or with SPECFIT/32TM global analysis system by Spectrum Software Associates (Marlborough, MA). Column chromatography was performed either on silica gel (60-400 mesh) or neutral alumina (Fluka, 0.05-0.15 mm, Brockmann Activity 1). Merck silica gel (60 F254) or neutral alumina (150 F254) sheets were used for thin layer chromatography (TLC). All rotor preparations were performed directly in the NMR tube using $\mathrm{CD}_{2} \mathrm{Cl}_{2}$ as solvent. Compounds $\mathbf{2},{ }^{1} \mathbf{4},{ }^{1} \mathbf{A 1},{ }^{2} \mathbf{B 2},{ }^{3} \mathbf{8},{ }^{4} \mathbf{9},{ }^{1}$ $\mathbf{1 2},{ }^{1} \mathbf{1 3},{ }^{5} \mathbf{S}^{1}$ were synthesized according to literature known procedures. 

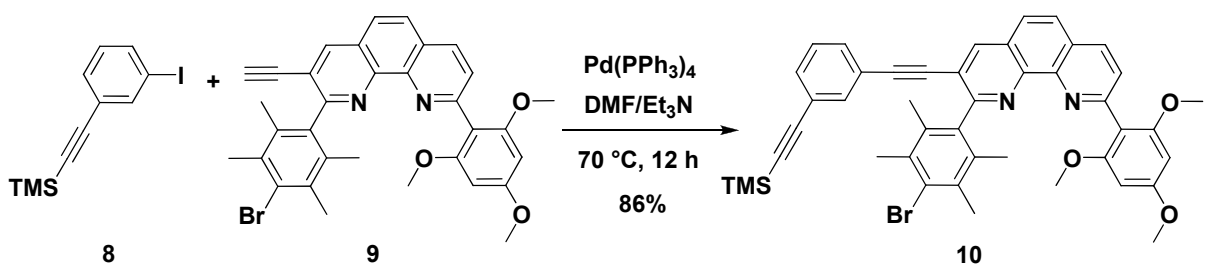

10

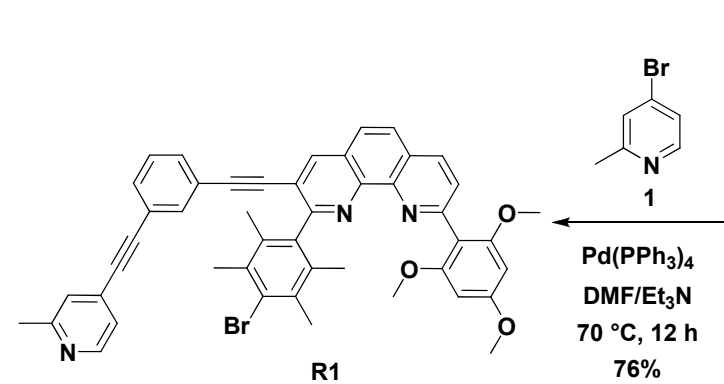

$\mathrm{K}_{2} \mathrm{CO}_{3}$ $\mathrm{THF} / \mathrm{MeOH}$
$\mathrm{rt}, 2 \mathrm{~h}, 93 \%$
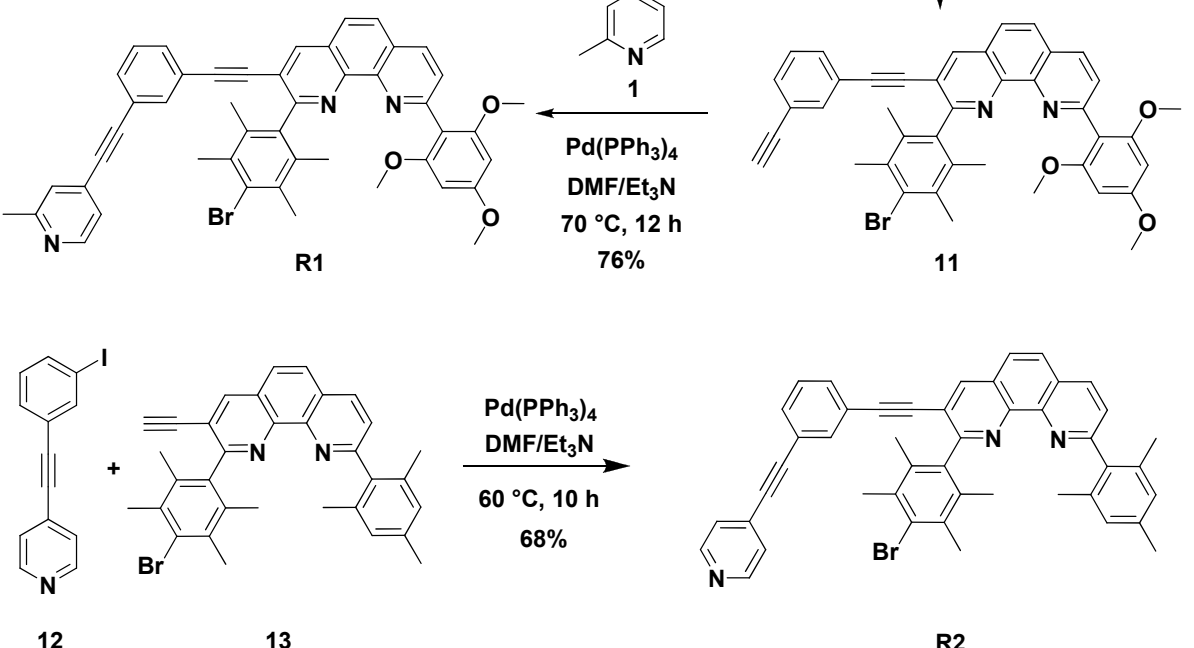

R2

Scheme 1. Synthesis of rotators $\mathbf{R} 1$ and $\mathbf{R 2}$.

\section{2-(4-Bromo-2,3,5,6-tetramethylphenyl)-9-(2,4,6-trimethoxyphenyl)-3-((3-((trimethylsilyl)-} ethynyl)phenyl)ethynyl)-1,10-phenanthroline (10)

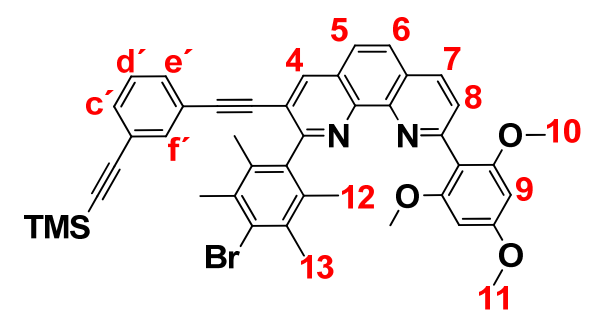

In an oven-dried $100 \mathrm{~mL}$ sealed tube, a mixture of ((3-iodophenyl)ethynyl)trimethylsilane (8) (207 $\mathrm{mg}, \quad 0.690 \quad \mathrm{mmol}) \quad$ and 2-(4-bromo-2,3,5,6-tetramethylphenyl)-3-ethynyl-9-(2,4,6trimethoxyphenyl)-1,10-phenanthroline (9) (200 mg, $0.344 \mathrm{mmol})$ were dissolved in dry DMF (20 $\mathrm{mL})$ and $\mathrm{Et}_{3} \mathrm{~N}(20 \mathrm{~mL})$ and degassed thoroughly. Then $\mathrm{Pd}\left(\mathrm{PPh}_{3}\right)_{4}(20.0 \mathrm{mg}, 17.3 \mu \mathrm{mol})$ was added and the mixture was refluxed at $70{ }^{\circ} \mathrm{C}$ for $12 \mathrm{~h}$ for completion of the coupling reaction. The reaction mixture was cooled down to room temperature and the solvents were removed. The residue was subjected to column chromatography ( $\mathrm{mg}$ of compound 10 as brown solid $(0.30 \mathrm{mmol}, 86 \%)$. Melting point $=186{ }^{\circ} \mathrm{C}$. IR $(\mathbf{K B r}): \tilde{v}=$ $530,612,645,665,684,722,758,794,810,845,895,924,948,989,993,1015,1038,1052,1073$, 
1130, 1155, 1204, 1224, 1249, 1335, 1384, 1414, 1457, 1491, 1535, 1586, 1608, 2157, 2203, 2225, 2836, $2955 \mathrm{~cm}^{-1} .{ }^{1} \mathbf{H}$ NMR (400 MHz, $\left.\mathbf{C D}_{2} \mathbf{C l}_{2}\right): \delta=0.27$ (s, 9H, TMS-H), 2.00 (s, 6H, 13-H), 2.49 (s, 6H, 12-H), 3.69 (s, 6H, 10-H), 3.87 (s, 3H, 11-H), 6.26 (s, 2H, 9-H), 7.09 (ddd, ${ }^{3} J=7.8 \mathrm{~Hz}$, $\left.{ }^{4} J=1.6 \mathrm{~Hz},{ }^{4} J=1.2 \mathrm{~Hz}, 1 \mathrm{H}, \mathrm{e}^{\prime} / \mathrm{c}^{\prime}-\mathrm{H}\right), 7.13\left(\mathrm{t},{ }^{3} J=1.2 \mathrm{~Hz}, 1 \mathrm{H}, \mathrm{f}^{\prime}-\mathrm{H}\right), 7.24\left(\mathrm{t},{ }^{3} J=7.8 \mathrm{~Hz}, 1 \mathrm{H}, \mathrm{d}^{\prime}-\right.$ H), 7.37 (ddd, $\left.{ }^{3} J=7.8 \mathrm{~Hz},{ }^{4} J=1.6 \mathrm{~Hz},{ }^{4} J=1.2 \mathrm{~Hz}, 1 \mathrm{H}, \mathrm{c}^{\prime} / \mathrm{e}^{\prime}-\mathrm{H}\right), 7.58\left(\mathrm{~d},{ }^{3} J=8.0 \mathrm{~Hz}, 1 \mathrm{H}, 8-\mathrm{H}\right)$, $7.86\left(\mathrm{~d},{ }^{3} J=8.8 \mathrm{~Hz}, 1 \mathrm{H}, 5 / 6-\mathrm{H}\right), 7.90\left(\mathrm{~d},{ }^{3} J=8.8 \mathrm{~Hz}, 1 \mathrm{H}, 6 / 5-\mathrm{H}\right), 8.27\left(\mathrm{~d},{ }^{3} J=8.0 \mathrm{~Hz}, 1 \mathrm{H}, 7-\mathrm{H}\right)$, 8.49 (s, 1H, 4-H) ppm. ${ }^{13} \mathbf{C}$ NMR (100 MHz, $\left.\mathbf{C D C l}_{3}\right): \delta=0.4,19.0,21.5,56.2,56.7,88.0,91.7$, $95.1,96.0,104.4,113,4,120.2,123.7,123.4,126.3,127.5,127.7,128.1,128.8,129.3,129.8$, $131.9,132.6,134.2,134.6,135.6,136.2,139.3,140.1,145.9,146.7,156.6,159.7,162.4,163.1$ ppm. ESI-MS: $m / z$ (\%) 753.9 (100) $[\mathbf{1 0}+\mathrm{H}]^{+}$. Elemental analysis: Calculated for $\mathrm{C}_{44} \mathrm{H}_{41} \mathrm{BrN}_{2} \mathrm{O}_{3} \mathrm{Si}: \mathrm{C}, 70.11 ; \mathrm{H}, 5.48 ; \mathrm{N}, 3.72$. Found: C, 69.88; H, 5.39; N, 3.46.

2-(4-Bromo-2,3,5,6-tetramethylphenyl)-3-((3-ethynylphenyl)ethynyl)-9-(2,4,6trimethoxyphenyl)-1,10-phenanthroline (11)

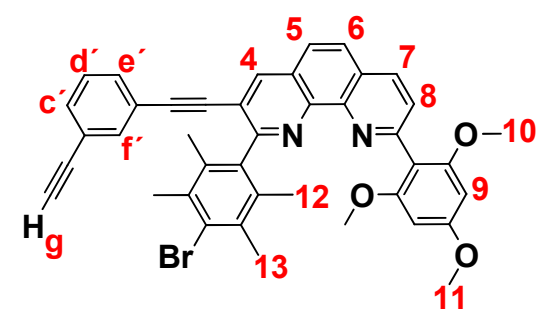

Compound 10 (200 mg, $0.265 \mathrm{mmol})$ was dissolved in THF (20 mL) and $\mathrm{MeOH}(20 \mathrm{~mL})$. Thereafter, aqueous $\mathrm{K}_{2} \mathrm{CO}_{3}\left(149 \mathrm{mg}, 1.08 \mathrm{mmol}\right.$ in $10 \mathrm{~mL}$ of deionized $\mathrm{H}_{2} \mathrm{O}$ ) was added and the mixture was allowed to stir at $\mathrm{rt}$ for $2 \mathrm{~h}$. The solvent was evaporated and the product was extracted in DCM (25 mL). After washing the organic layer with deionized water $(50 \mathrm{~mL})$, the organic layer was removed and dried over anhydrous $\mathrm{MgSO}_{4}$. The evaporation of solvent afforded compound 11 as colorless solid (168 mg, $0.246 \mathrm{mmol}, 93 \%)$. Melting point $=211^{\circ} \mathrm{C}$. IR (KBr): $\tilde{v}=533,568$, 614, 641, 665, 689, 722, 769, 734, 805, 818, 843, 892, 906, 947, 988, 1015, 1039, 1052, 1072, 1129, 1158, 1184, 1204, 1225, 1277, 1334, 1384, 1413, 1435, 1464, 1492, 1537, 1586, 1608, 2099, 2213, 2838, 2932, 2991, $3162 \mathrm{~cm}^{-1} .{ }^{\mathbf{1}} \mathbf{H}$ NMR (400 MHz, $\left.\mathbf{C D}_{\mathbf{2}} \mathbf{C l}_{2}\right): \delta=2.01(\mathrm{~s}, 6 \mathrm{H}, 13-\mathrm{H}), 2.49$ (s, $6 \mathrm{H}, 12-\mathrm{H}), 3.18$ (s, 1H, g-H), 3.69 (s, 6H, 10-H), 3.87 (s, 3H, 11-H), 6.26 (s, 2H, 9-H), 7.10 (ddd, ${ }^{3} J$ $\left.=7.8 \mathrm{~Hz},{ }^{4} J=1.6 \mathrm{~Hz},{ }^{4} J=1.2 \mathrm{~Hz}, 1 \mathrm{H}, \mathrm{e}^{\prime} / \mathrm{c}^{\prime}-\mathrm{H}\right), 7.21\left(\mathrm{t},{ }^{4} J=1.2 \mathrm{~Hz}, 1 \mathrm{H}, \mathrm{f}^{\prime}-\mathrm{H}\right), 7.26\left(\mathrm{t},{ }^{3} \mathrm{~J}=7.8 \mathrm{~Hz}\right.$, $\left.1 \mathrm{H}, \mathrm{d}^{\prime}-\mathrm{H}\right), 7.42\left(\mathrm{ddd},{ }^{3} J=7.8 \mathrm{~Hz},{ }^{4} J=1.6 \mathrm{~Hz},{ }^{4} J=1.2 \mathrm{~Hz}, 1 \mathrm{H}, \mathrm{c}^{\prime} / \mathrm{e}^{\prime}-\mathrm{H}\right), 7.59\left(\mathrm{~d},{ }^{3} J=8.0 \mathrm{~Hz}, 1 \mathrm{H}\right.$, 8-H), $7.86\left(\mathrm{~d},{ }^{3} J=8.8 \mathrm{~Hz}, 1 \mathrm{H}, 5 / 6-\mathrm{H}\right), 7.91\left(\mathrm{~d},{ }^{3} J=8.8 \mathrm{~Hz}, 1 \mathrm{H}, 6 / 5-\mathrm{H}\right), 8.27\left(\mathrm{~d},{ }^{3} J=8.0 \mathrm{~Hz}, 1 \mathrm{H}, 7-\right.$ H), 8.50 (s, 1H, 4-H) ppm. ${ }^{13} \mathbf{C}$ NMR (100 MHz, $\left.\mathbf{C D C l}_{3}\right): \delta=18.6,21.1,55.9,56.3,78.3,82.8$, $87.7,91.3,94.5,119.7,122.9,123.5,126.0,127.2,127.3,127.7,128.4,129.0,129.4,132.0,132.5$, 
133.8, 134.3, 135.4, 135.8, 139.0, 139.7, 145.2, 145.6, 146.4, 156.2, 159.3, 162.1, 162.7 ppm. ESIMS: $m / z(\%) 681.7(100)[11+\mathrm{H}]^{+}$. Elemental analysis: Calcd. for $\mathrm{C}_{41} \mathrm{H}_{33} \mathrm{BrN}_{2} \mathrm{O}_{3} \cdot \mathrm{H}_{2} \mathrm{O}: \mathrm{C}, 70.39$; H, 5.04; N, 4.0. Found: C, 70.39; H, 4.69; N, 3.93.

2-(4-Bromo-2,3,5,6-tetramethylphenyl)-3-((3-((2-methylpyridin-4-yl)ethynyl)phenyl)ethynyl)9-(2,4,6-trimethoxyphenyl)-1,10-phenanthroline (R1)

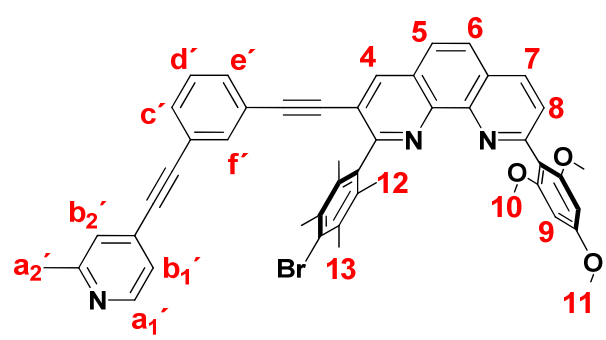

In an oven-dried $100 \mathrm{~mL}$ tube, compounds 11 (70.0 mg, $103 \mu \mathrm{mol})$ and 1 (145 mg, $843 \mu \mathrm{mol})$ were dissolved in dry DMF $(20 \mathrm{~mL})$ and $\mathrm{Et}_{3} \mathrm{~N}(20 \mathrm{~mL})$ and degassed thoroughly. Then $\mathrm{Pd}_{(}\left(\mathrm{PPh}_{3}\right)_{4}(20.0$ $\mathrm{mg}, 17.3 \mu \mathrm{mol}$ ) was added and the mixture was refluxed at $70{ }^{\circ} \mathrm{C}$ for $12 \mathrm{~h}$. The reaction mixture was cooled down to $\mathrm{rt}$ and the solvents were removed. The residue was subjected to column chromatography (silica gel, ethyl acetate $/ \mathrm{CH}_{2} \mathrm{Cl}_{2}=3: 7, R_{\mathrm{f}}=0.2$ ) to afford $60 \mathrm{mg}$ of compound $\mathbf{R} \mathbf{1}$ as colorless solid $(77.6 \mu \mathrm{mol}, 76 \%)$. Melting point $=184{ }^{\circ} \mathrm{C}$. IR (KBr): $\tilde{v}=528,566,579,684$, 753, 791, 857, 893, 1087, 1128, 1157, 1205, 1226, 1335, 1384, 1414, 1458, 1466, 1602, 2025, 2219, 2841, 2932, $3007 \mathrm{~cm}^{-1} .{ }^{\mathbf{1}} \mathbf{H}$ NMR (400 MHz, $\left.\mathbf{C D}_{2} \mathbf{C l}_{2}\right): \delta=2.02$ (s, 6H, 13-H), 2.50 (s, 6H, 12-H), 2.57 (s, 3H, a $\left.{ }^{\prime}-\mathrm{H}\right), 3.71(\mathrm{~s}, 6 \mathrm{H}, 10-\mathrm{H}), 3.87$ (s, 3H, 11-H), 6.26 (s, 2H, 9-H), 7.17 (ddd, ${ }^{3} J=$ $\left.7.8 \mathrm{~Hz},{ }^{4} J=1.6 \mathrm{~Hz},{ }^{4} J=1.2 \mathrm{~Hz}, 1 \mathrm{H}, \mathrm{e}^{\prime} / \mathrm{c}^{\prime}-\mathrm{H}\right), 7.21\left(\mathrm{t},{ }^{4} J=1.2 \mathrm{~Hz}, 1 \mathrm{H}, \mathrm{f}^{\prime}-\mathrm{H}\right), 7.27\left(\mathrm{~d},{ }^{3} J=5.6 \mathrm{~Hz}\right.$, $\left.1 \mathrm{H}, \mathrm{b}_{1}{ }^{\prime}-\mathrm{H}\right), 7.32\left(\mathrm{t},{ }^{3} J=7.8 \mathrm{~Hz}, 1 \mathrm{H}, \mathrm{d}^{\prime}-\mathrm{H}\right), 7.34$ (s, $\left.1 \mathrm{H}, \mathrm{b}_{2}{ }^{\prime}-\mathrm{H}\right), 7.49$ (ddd, ${ }^{3} J=7.8 \mathrm{~Hz},{ }^{4} J=1.6 \mathrm{~Hz}$, $\left.{ }^{4} J=1.2 \mathrm{~Hz}, 1 \mathrm{H}, \mathrm{c}^{\prime} / \mathrm{e}^{\prime}-\mathrm{H}\right), 7.67\left(\mathrm{~d},{ }^{3} J=8.0 \mathrm{~Hz}, 1 \mathrm{H}, 8-\mathrm{H}\right), 7.89\left(\mathrm{~d},{ }^{3} J=8.8 \mathrm{~Hz}, 1 \mathrm{H}, 5 / 6-\mathrm{H}\right), 7.94$ (d, $\left.{ }^{3} J=8.8 \mathrm{~Hz}, 1 \mathrm{H}, 6 / 5-\mathrm{H}\right), 8.34\left(\mathrm{~d},{ }^{3} J=8.0 \mathrm{~Hz}, 1 \mathrm{H}, 7-\mathrm{H}\right), 8.49\left(\mathrm{~d},{ }^{3} J=5.6 \mathrm{~Hz}, 1 \mathrm{H}, \mathrm{a}_{1}{ }^{\prime}-\mathrm{H}\right), 8.52(\mathrm{~s}$, 1H, 4-H) ppm. ${ }^{13} \mathbf{C}$ NMR (100 MHz, $\left.\mathbf{C D C l}_{3}\right): \delta=18.6,21.1,24.2,55.9,56.4,87.8,87.8,88.0$, 91.4, 92.7, 94.7, 120.0, 123.1, 123.6, 125.6, 126.1, 127.2, 127.4, 127.7, 128.4, 129.2, 129.4, 131.9, $132.1,132.3,133.8,134.3,135.2,136.4,139.0,139.6,139.7,144.9,145.6,149.0,155.8,158.9$, 159.5, 162.3, 162.8 ppm. ESI-MS: $m / z(\%) 774.5(100)[\text { R1 }+\mathrm{H}]^{+}$. Elemental analysis: Calcd. for $\mathrm{C}_{47} \mathrm{H}_{38} \mathrm{BrN}_{3} \mathrm{O}_{3} \bullet \mathrm{H}_{2} \mathrm{O}: \mathrm{C}, 71.39 ; \mathrm{H}, 5.10 ; \mathrm{N}$, 5.31. Found: C, 71.05; H, 4.86; N, 5.31. 


\section{1,10-phenanthroline (R2)}

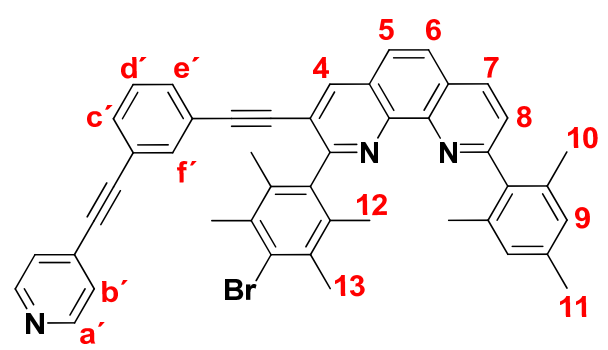

A mixture of 4-((3-iodophenyl)ethynyl)pyridine (12) (120 mg, $0.393 \mathrm{mmol})$, 2-(4-bromo-2,3,5,6tetramethylphenyl)-3-ethynyl-9-mesityl-1,10-phenanthroline (13) (70.0 mg, $0.131 \mathrm{mmol})$ were taken in oven-dried $100 \mathrm{~mL}$ tube and dissolved in dry DMF $(20 \mathrm{~mL})$ and $\mathrm{Et}_{3} \mathrm{~N}(20 \mathrm{~mL})$. The solution was degassed thoroughly and refluxed for $10 \mathrm{~h}$ at $60{ }^{\circ} \mathrm{C}$ and then evaporated to dryness. The crude product was purified by column chromatography ( silica gel, $\mathrm{CH}_{2} \mathrm{Cl}_{2}, R_{\mathrm{f}}=0.3$ ) providing $63.0 \mathrm{mg}$ of $\mathbf{R 2}$ as colorless solid $(88.6 \mu \mathrm{mol}, 68 \%)$. Melting point $=197{ }^{\circ} \mathrm{C}$; IR (KBr): $\tilde{v}=533$, 639, 685, 795, 818, 850, 895, 921, 988, 1144, 1166, 1213, 1384, 1458, 1475, 1536, 1595, 1938, 2212, 2918, $3052 \mathrm{~cm}^{-1}$. ${ }^{1} \mathbf{H}$ NMR (400 MHz, $\left.\mathbf{C D}_{2} \mathbf{C l}_{2}\right): \delta=2.01$ (s, 6H, 13-H), 2.04 (s, 6H, 10-H), $2.34(\mathrm{~s}, 3 \mathrm{H}, 11-\mathrm{H}), 2.50(\mathrm{~s}, 6 \mathrm{H}, 12-\mathrm{H}), 6.96(\mathrm{~s}, 2 \mathrm{H}, 9-\mathrm{H}), 7.17\left(\mathrm{ddd},{ }^{3} J=7.8 \mathrm{~Hz},{ }^{4} J=1.6 \mathrm{~Hz},{ }^{4} J=\right.$ $\left.1.2 \mathrm{~Hz}, 1 \mathrm{H}, \mathrm{e}^{\prime} / \mathrm{c}^{\prime}-\mathrm{H}\right), 7.22\left(\mathrm{t},{ }^{4} J=1.2 \mathrm{~Hz}, 1 \mathrm{H}, \mathrm{f}^{\prime}-\mathrm{H}\right), 7.33\left(\mathrm{t},{ }^{3} J=7.8 \mathrm{~Hz}, 1 \mathrm{H}, \mathrm{d}^{\prime}-\mathrm{H}\right), 7.44\left(\mathrm{~d},{ }^{3} J=6.0\right.$ $\left.\mathrm{Hz}, 2 \mathrm{H}, \mathrm{b}^{\prime}-\mathrm{H}\right), 7.50\left(\mathrm{ddd},{ }^{3} J=7.8 \mathrm{~Hz},{ }^{4} J=1.6 \mathrm{~Hz},{ }^{4} J=1.2 \mathrm{~Hz}, 1 \mathrm{H}, \mathrm{c}^{\prime} / \mathrm{e}^{\prime}-\mathrm{H}\right), 7.58\left(\mathrm{~d},{ }^{3} J=8.0 \mathrm{~Hz}\right.$, $1 \mathrm{H}, 8-\mathrm{H}), 7.89\left(\mathrm{~d},{ }^{3} J=8.8 \mathrm{~Hz}, 1 \mathrm{H}, 5 / 6-\mathrm{H}\right), 7.94\left(\mathrm{~d},{ }^{3} J=8.8 \mathrm{~Hz}, 1 \mathrm{H}, 6 / 5-\mathrm{H}\right), 8.34\left(\mathrm{~d},{ }^{3} J=8.0 \mathrm{~Hz}\right.$, 1H, 7-H), 8.52 (s, 1H, 4-H), $8.62\left(\mathrm{~d},{ }^{3} J=6.0 \mathrm{~Hz}, 2 \mathrm{H}, \mathrm{a}^{\prime}-\mathrm{H}\right) \mathrm{ppm} .{ }^{13} \mathbf{C}$ NMR (100 MHz, CDCl $): \delta$ $=18.6,21.5,21.2,21.3,87.7,88.0,92.8,94.6,119.8,123.0,123.6,125.3,125.9,126.0,127.3$, $127.6,128.1,128.7,129.2,129.4,131.3,132.1,132.2,133.9,134.2,135.2,136.1,136.5,138.0$, 138.4, 138.8, 139.7, 145.5, 146.4, 150.3, 161.0, 162.8 ppm. ESI-MS: $m / z(\%) 712.5$ (100) [R2 + $\mathrm{H}]^{+}$. Elemental analysis: Calcd. for $\mathrm{C}_{46} \mathrm{H}_{36} \mathrm{BrN}_{3}$ : C, 77.74; H, 5.11; N, 5.91. Found: C, 77.52; H, $4.74 ; \mathrm{N}, 5.83$. 


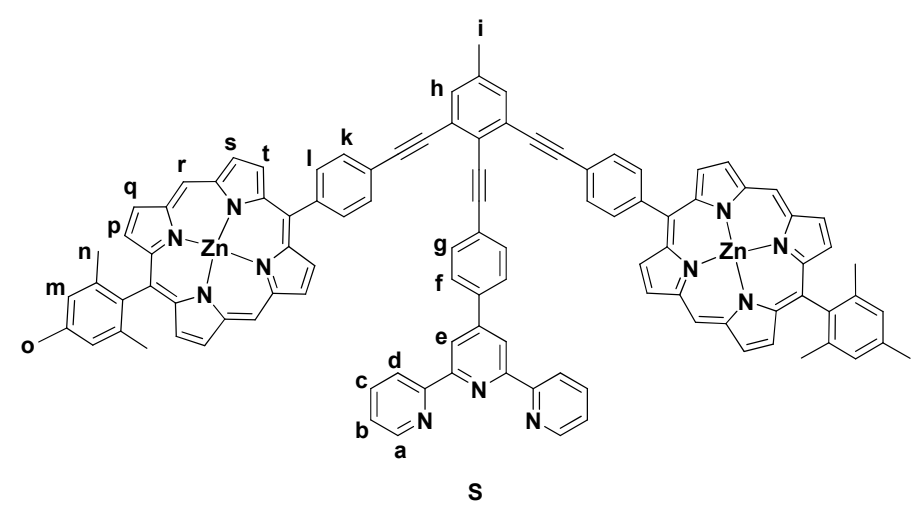

Melting point: $>250{ }^{\circ} \mathrm{C}$. IR (KBr): $\tilde{v}=564,608,634,702,720,734,785,813,833,858,906$, 995, 1059, 1142, 1212, 1287, 1315, 1391, 1439, 1496, 1521, 1608, 2227, $2915 \mathrm{~cm}^{-1}$. ${ }^{\mathbf{1}} \mathbf{H}$ NMR (400 MHz, $\left.\mathbf{C D}_{2} \mathbf{C l}_{2}\right): \delta=1.82(\mathrm{~s}, 12 \mathrm{H}, \mathrm{n}-\mathrm{H}), 2.62(\mathrm{~s}, 3 \mathrm{H}, \mathrm{i}-\mathrm{H}), 2.70$ (s, 6H, o-H), 7.11 (ddd, ${ }^{3} J=8.0$ $\left.\mathrm{Hz},{ }^{3} J=5.0 \mathrm{~Hz},{ }^{4} J=1.2 \mathrm{~Hz}, 2 \mathrm{H}, \mathrm{b}-\mathrm{H}\right), 7.38(\mathrm{~s}, 4 \mathrm{H}, \mathrm{m}-\mathrm{H}), 7.70\left(\mathrm{td},{ }^{3} J=8.0 \mathrm{~Hz},{ }^{4} J=1.2 \mathrm{~Hz}, 2 \mathrm{H}, \mathrm{c}-\right.$ H), 7.73 (s, 2H, h-H), $8.11\left(\mathrm{~d},{ }^{3} J=8.4 \mathrm{~Hz}, 2 \mathrm{H}, \mathrm{g} / \mathrm{f}-\mathrm{H}\right), 8.15\left(\mathrm{~d},{ }^{3} J=8.4 \mathrm{~Hz}, 2 \mathrm{H}, \mathrm{f} / \mathrm{g}-\mathrm{H}\right), 8.19\left(\mathrm{~d},{ }^{3} J=\right.$ $8.0 \mathrm{~Hz}, 4 \mathrm{H}, \mathrm{k}-\mathrm{H}), 8.33\left(\mathrm{ddd},{ }^{3} J=5.0 \mathrm{~Hz},{ }^{4} \mathrm{~J}=1.2 \mathrm{~Hz},{ }^{5} \mathrm{~J}=0.8 \mathrm{~Hz}, 2 \mathrm{H}, \mathrm{a}-\mathrm{H}\right), 8.38\left(\mathrm{~d},{ }^{3} J=8.0 \mathrm{~Hz}\right.$, $4 \mathrm{H}, \mathrm{l}-\mathrm{H}), 8.47$ (ddd, $\left.{ }^{3} J=8.0 \mathrm{~Hz},{ }^{4} J=1.2 \mathrm{~Hz},{ }^{5} J=0.8 \mathrm{~Hz}, 2 \mathrm{H}, \mathrm{d}-\mathrm{H}\right), 8.75$ (s, 2H, e-H), $8.95\left(\mathrm{~d},{ }^{3} J=\right.$ $4.4 \mathrm{~Hz}, 4 \mathrm{H}, \mathrm{p}-\mathrm{H}), 9.23$ (d, $\left.{ }^{3} J=4.4 \mathrm{~Hz}, 4 \mathrm{H}, \mathrm{t}-\mathrm{H}\right), 9.43$ (d, $\left.{ }^{3} J=4.4 \mathrm{~Hz}, 4 \mathrm{H}, \mathrm{q}-\mathrm{H}\right), 9.51$ (d, ${ }^{3} J=4.4 \mathrm{~Hz}$, 4H, s-H), 10.28 (s, 4H, r-H). ${ }^{13}$ C NMR (100 MHz, d8-THF:CD $\mathbf{d}_{\mathbf{2}} \mathbf{C l}_{\mathbf{2}}=\mathbf{8 : 2}$ ): $\delta=20.6,21.0,21.5$, 89.2, 89.5, 94.3, 97.3, 105.6, 117.7, 118.4, 118.6, 120.9, 122.5, 124.0, 125.0, 125.6, 126.8, 127.7, $127.9,130.1,130.7,131.7,131.9,132.1,132.7,132.7,135.3,136.7,137.5,138.9,139.0,139.3$, 139.8, 144.5, 149.2, 149.3, 149.8, 149.8, 149.9, 149.9, 156.1, 156.4 ppm. ESI-MS: $m / z(\%) 1604.7$ (100) $[\mathrm{S}+\mathrm{H}]^{+}$. Elemental analysis: Calcd. for $\mathrm{C}_{104} \mathrm{H}_{69} \mathrm{~N}_{11} \mathrm{Zn}_{2} \cdot \mathrm{H}_{2} \mathrm{O}: \mathrm{C}, 77.03 ; \mathrm{H}, 4.41 ; \mathrm{N}$, 9.50.Found: C, 77.04; H, 4.15; N, 9.40.

\section{Synthesis and characterization of complexes}

a) Model Complex $\mathbf{C 1}=[\mathbf{3} \rightarrow \mathbf{4}]$

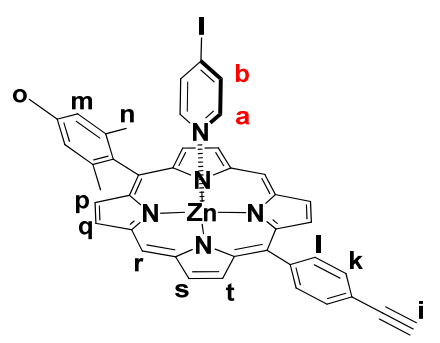

In an NMR tube, zinc porphyrin $4(0.456 \mathrm{mg}, 0.770 \mu \mathrm{mol})$ and 4-iodopyridine (3) (0.158 $\mathrm{mg}, 0.771$ $\mu \mathrm{mol})$ were dissolved in $500 \mu \mathrm{L}$ of $\mathrm{CD}_{2} \mathrm{Cl}_{2}$ and NMR spectra were recorded. Yield: quantitative. 
Melting point: $>200{ }^{\circ} \mathrm{C}$; IR (KBr): $\tilde{v}=558,614,697,721,787,809,838,886,909,995,1057$, 1121, 1143,1158, 1182, 1209, 1228, 1288, 1313, 1374, 1395, 1437, 1522, 1559, 1612, 2137, 2915, 3051, $3079 \mathrm{~cm}^{-1}$. ${ }^{1} \mathbf{H}$ NMR (400 MHz, $\left.\mathbf{C D}_{\mathbf{2}} \mathbf{C l}_{2}\right): \delta=1.77$ (s, 6H, n-H), 2.66 (s, 3H, o-H), 3.02 (brs, 2H, a-H), 3.40 (s, 1H, i-H), $6.12\left(\mathrm{~d},{ }^{3} J=5.6 \mathrm{~Hz}, 2 \mathrm{H}, \mathrm{b}-\mathrm{H}\right), 7.33$ (s, 2H, m-H), 7.93 (d, ${ }^{3} J=8.0 \mathrm{~Hz}$, $2 \mathrm{H}, \mathrm{k}-\mathrm{H}), 8.25\left(\mathrm{~d},{ }^{3} \mathrm{~J}=8.0 \mathrm{~Hz}, 2 \mathrm{H}, \mathrm{l}-\mathrm{H}\right), 8.88\left(\mathrm{~d},{ }^{3} \mathrm{~J}=4.5 \mathrm{~Hz}, 2 \mathrm{H}, \mathrm{p}-\mathrm{H}\right), 9.06\left(\mathrm{~d},{ }^{3} J=4.5 \mathrm{~Hz}, 2 \mathrm{H}, \mathrm{t}-\right.$ H), $9.36\left(\mathrm{~d},{ }^{3} J=4.5 \mathrm{~Hz}, 2 \mathrm{H}, \mathrm{q}-\mathrm{H}\right), 9.41\left(\mathrm{~d},{ }^{3} J=4.5 \mathrm{~Hz}, 2 \mathrm{H}, \mathrm{s}-\mathrm{H}\right), 10.21$ (s, 2H, r-H) ppm.

b) Model complex $\mathbf{C 2}=[\mathrm{Cu}(\mathbf{1})(\mathbf{2})]^{+}$

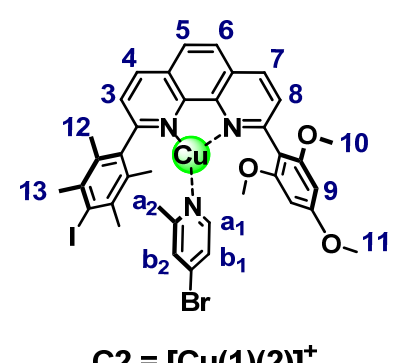

In an NMR tube, $\left[\mathrm{Cu}\left(\mathrm{CH}_{3} \mathrm{CN}\right)_{4}\right] \mathrm{PF}_{6}(0.868 \mathrm{mg}, 2.33 \mu \mathrm{mol})$, ligands $2(1.41 \mathrm{mg}, 2.33 \mu \mathrm{mol})$ and 4bromo-2-methylpyridine (1) $(0.401 \mathrm{mg}, 2.33 \mu \mathrm{mol})$ were dissolved in $500 \mu \mathrm{L}$ of $\mathrm{CD}_{2} \mathrm{Cl}_{2}$. Yield by NMR: quantitative. ${ }^{1} \mathbf{H}$ NMR ( $\mathbf{C D}_{2} \mathbf{C l}_{2}, 400$ MHz): $\delta 1.95$ (s, 6H, 13-H), 2.07 (s, 3H, a $\left.2-H\right), 2.37$ (s, $6 \mathrm{H}, 12-\mathrm{H}), 3.61(\mathrm{~s}, 6 \mathrm{H}, 10-\mathrm{H}), 3.75(\mathrm{~s}, 3 \mathrm{H}, 11-\mathrm{H}), 6.01(\mathrm{~s}, 2 \mathrm{H}, 9-\mathrm{H}), 7.22\left(\mathrm{dd},{ }^{3} J=6.0 \mathrm{~Hz},{ }^{4} \mathrm{~J}=2.0\right.$ $\left.\mathrm{Hz}, 1 \mathrm{H}, \mathrm{b}_{1}-\mathrm{H}\right), 7.35\left(\mathrm{~d},{ }^{4} J=2.0 \mathrm{~Hz}, 1 \mathrm{H}, \mathrm{b}_{2}-\mathrm{H}\right), 7.55\left(\mathrm{~d},{ }^{3} J=6.0 \mathrm{~Hz}, 1 \mathrm{H}, \mathrm{a}_{1}-\mathrm{H}\right), 7.87\left(\mathrm{~d},{ }^{3} J=8.4 \mathrm{~Hz}\right.$, $1 \mathrm{H}, 8-\mathrm{H}), 7.96\left(\mathrm{~d},{ }^{3} J=8.4 \mathrm{~Hz}, 1 \mathrm{H}, 3-\mathrm{H}\right), 8.13(\mathrm{~s}, 2 \mathrm{H}, 5+6-\mathrm{H}), 8.59\left(\mathrm{~d},{ }^{3} J=8.4 \mathrm{~Hz}, 1 \mathrm{H}, 7-\mathrm{H}\right), 8.68$ $\left(\mathrm{d},{ }^{3} \mathrm{~J}=8.4 \mathrm{~Hz}, 1 \mathrm{H}, 4-\mathrm{H}\right)$ ppm. ESI-MS: $\left.m / z(\%)=839.7(100)[\mathrm{Cu}(\mathbf{1})(\mathbf{2})]^{+}\right]$.

c) Model complex $\mathbf{C 3}=[\mathbf{1} \rightarrow \mathbf{4}]$

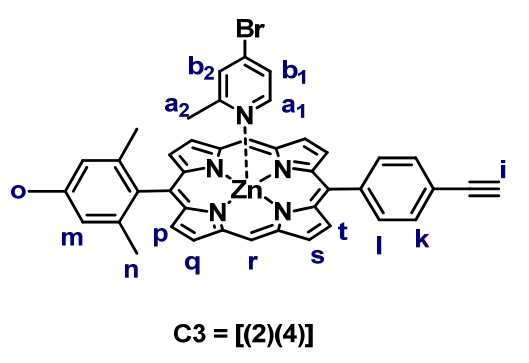

In an NMR tube, zinc porphyrin $4(0.456 \mathrm{mg}, 0.770 \mu \mathrm{mol})$ and $1(0.133 \mathrm{mg}, 0.771 \mu \mathrm{mol})$ were dissolved in $500 \mu \mathrm{L}$ of $\mathrm{CD}_{2} \mathrm{Cl}_{2}$ and NMR spectra were recorded. Yield: quantitative. ${ }^{1} \mathbf{H}$ NMR (400 MHz, CD $\left.\mathbf{C l}_{2}\right): \delta=1.81(\mathrm{~s}, 6 \mathrm{H}, \mathrm{n}-\mathrm{H}), 1.93$ (s, 3H, a $\left.2-\mathrm{H}\right)^{*}, 2.66$ (s, 3H, m2-H), 3.40 (s, 1H, iH), $7.04\left(\mathrm{dd},{ }^{3} J=4.0 \mathrm{~Hz},{ }^{4} J=1.2 \mathrm{~Hz}, 1 \mathrm{H}, \mathrm{b}_{1}-\mathrm{H}\right), 7.04\left(\mathrm{~d},{ }^{4} \mathrm{~J}=1.2 \mathrm{~Hz}, 1 \mathrm{H}, \mathrm{b}_{2}-\mathrm{H}\right), 7.34$ (s, 2H, mH), $7.39\left(\mathrm{~d},{ }^{3} J=4.0 \mathrm{~Hz}, 1 \mathrm{H}, \mathrm{a}_{1}-\mathrm{H}\right), 7.95\left(\mathrm{~d},{ }^{3} J=8.0 \mathrm{~Hz}, 2 \mathrm{H}, \mathrm{k}-\mathrm{H}\right), 8.25\left(\mathrm{~d},{ }^{3} J=8.0 \mathrm{~Hz}, 2 \mathrm{H}, 1-\mathrm{H}\right)$, 
$8.94\left(\mathrm{~d},{ }^{3} J=4.5 \mathrm{~Hz}, 2 \mathrm{H}, \mathrm{p}-\mathrm{H}\right), 9.11\left(\mathrm{~d},{ }^{3} J=4.5 \mathrm{~Hz}, 2 \mathrm{H}, \mathrm{t}-\mathrm{H}\right), 9.42\left(\mathrm{~d},{ }^{3} J=4.5 \mathrm{~Hz}, 2 \mathrm{H}, \mathrm{q}-\mathrm{H}\right), 9.47$ $\left(\mathrm{d},{ }^{3} \mathrm{~J}=4.5 \mathrm{~Hz}, 2 \mathrm{H}, \mathrm{s}-\mathrm{H}\right), 10.30(\mathrm{~s}, 2 \mathrm{H}, \mathrm{r}-\mathrm{H}) \mathrm{ppm}$. * Shift depends on concentration.

d) Model complex $\mathbf{C 4}=[\mathrm{Cu}(\mathbf{2})(\mathbf{3})]^{+}$

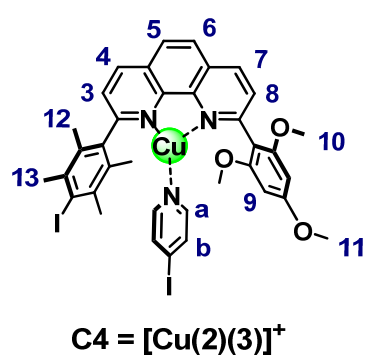

In an NMR tube, ligands $2(1.41 \mathrm{mg}, 2.33 \mu \mathrm{mol})$ and $\mathbf{3}(0.478 \mathrm{mg}, 2.33 \mu \mathrm{mol})$ as well as $\left[\mathrm{Cu}\left(\mathrm{CH}_{3} \mathrm{CN}\right)_{4}\right] \mathrm{PF}_{6}(0.422 \mathrm{mg}, 2.33 \mu \mathrm{mol})$ were dissolved in $500 \mu \mathrm{L}$ of $\mathrm{CD}_{2} \mathrm{Cl}_{2}$. Yield by NMR: quantitative. ${ }^{1} \mathbf{H}$ NMR (CD $\mathbf{C l}_{2}, 400$ MHz): $\delta 1.97(\mathrm{~s}, 6 \mathrm{H}, 13-\mathrm{H}), 2.47$ (s, 6H, 12-H), $3.63(\mathrm{~s}, 6 \mathrm{H}$, $10-\mathrm{H}), 3.83$ (s, 3H, 11-H), 6.13 (s, 2H, 9-H), 7.11 (brs, 2H, b-H), 7.63 (brs, 2H, a-H), 7.86 (d, ${ }^{3} J=$ $8.4 \mathrm{~Hz}, 1 \mathrm{H}, 8-\mathrm{H}), 7.97\left(\mathrm{~d},{ }^{3} J=8.4 \mathrm{~Hz}, 1 \mathrm{H}, 3-\mathrm{H}\right), 8.12(\mathrm{~s}, 2 \mathrm{H}, 5+6-\mathrm{H}), 8.58\left(\mathrm{~d},{ }^{3} J=8.4 \mathrm{~Hz}, 1 \mathrm{H}, 7-\right.$ H), $8.68\left(\mathrm{~d},{ }^{3} J=8.4 \mathrm{~Hz}, 1 \mathrm{H}, 4-\mathrm{H}\right)$ ppm. ESI-MS: $m / z(\%)=871.7(100)\left[[\mathrm{Cu}(\mathbf{2})(\mathbf{3})]^{+}\right]$.

e) Model complex $\mathbf{C 5}=[\operatorname{Zn}(\mathbf{2})(\mathbf{6})]^{2+}$

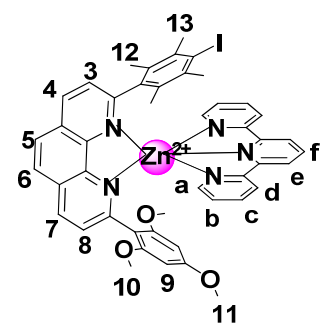

In an NMR tube, phenanthroline $2(0.469 \mathrm{mg}, 0.776 \mu \mathrm{mol})$, terpyridine (6) $(0.181 \mathrm{mg}, 0.776 \mu \mathrm{mol})$, and $\mathrm{Zn}(\mathrm{OTf})_{2}(0.282 \mathrm{mg}, 0.776 \mu \mathrm{mol})$ were dissolved in $500 \mu \mathrm{L}$ of $\mathrm{CD}_{2} \mathrm{Cl}_{2}: \mathrm{CD}_{3} \mathrm{CN}=10: 1$. After heating the sample at $50{ }^{\circ} \mathrm{C}$ for $2 \mathrm{~h}$, NMR spectra were recorded showing exclusive formation of the zinc HETTAP complex (>95\%). IR (KBr): $\widetilde{v}=580,613,637,779,1031,1129,1159,1227$, 1268, 1382, 1430, 1460, 1560, 1603, 2221, 2853, $2924 \mathrm{~cm}^{-1} .{ }^{\mathbf{1}} \mathbf{H}$ NMR (400 MHz, $\mathbf{C D}_{\mathbf{2}} \mathbf{C l}_{2}: \mathbf{C D}_{\mathbf{3}} \mathbf{C N}$ = 10:1): $\delta=0.86(\mathrm{~s}, 6 \mathrm{H}, 13-\mathrm{H}), 1.94(\mathrm{~s}, 6 \mathrm{H}, 12-\mathrm{H}), 2.91(\mathrm{~s}, 6 \mathrm{H}, 10-\mathrm{H}), 3.48(\mathrm{~s}, 3 \mathrm{H}, 11-\mathrm{H}), 5.58(\mathrm{~s}$, 2H, 9-H), 7.49 (ddd, $\left.{ }^{3} J=7.6 \mathrm{~Hz},{ }^{3} J=5.6 \mathrm{~Hz},{ }^{4} J=1.6 \mathrm{~Hz}, 2 \mathrm{H}, \mathrm{b}-\mathrm{H}\right), 7.59$ (ddd, ${ }^{3} J=5.6 \mathrm{~Hz},{ }^{4} J=1.6$ $\left.\mathrm{Hz},{ }^{5} J=0.8 \mathrm{~Hz}, 2 \mathrm{H}, \mathrm{a}-\mathrm{H}\right), 7.87\left(\mathrm{~d},{ }^{3} J=8.4 \mathrm{~Hz}, 1 \mathrm{H}, 8-\mathrm{H}\right), 8.08\left(\mathrm{~d},{ }^{3} J=8.4 \mathrm{~Hz}, 1 \mathrm{H}, 3-\mathrm{H}\right), 8.25\left(\mathrm{td},{ }^{3} J\right.$ $\left.=7.6 \mathrm{~Hz},{ }^{4} \mathrm{~J}=1.6 \mathrm{~Hz}, 2 \mathrm{H}, \mathrm{c}-\mathrm{H}\right), 8.41-8.49$ (m, 6H, d-, e-, 5- \& 6-H), 8.59 (t, $\left.{ }^{3} J=8.2 \mathrm{~Hz}, 1 \mathrm{H}, \mathrm{f}-\mathrm{H}\right)$, $8.97\left(\mathrm{~d},{ }^{3} J=8.4 \mathrm{~Hz}, 1 \mathrm{H}, 7-\mathrm{H}\right), 9.00\left(\mathrm{~d},{ }^{3} J=8.4 \mathrm{~Hz}, 1 \mathrm{H}, 4-\mathrm{H}\right) \mathrm{ppm}$. ESI-MS: $m / z(\%) 450.8(20)$ $[$ C5 $], \quad 1049.8$ (100) $\quad[$ C5 + OTf $]$. Elemental analysis: Calculated for 
$\mathrm{C}_{48} \mathrm{H}_{40} \mathrm{~F}_{6} \mathrm{IN}_{5} \mathrm{O}_{9} \mathrm{~S}_{2} \mathrm{Zn} \bullet 0.7 \mathrm{CH}_{2} \mathrm{Cl}_{2}: \mathrm{C}, 46.39 ; \mathrm{H}, 3.31 ; \mathrm{N}, 5.55 ; \mathrm{S}, 5.09$. Found: $\mathrm{C}, 46.74 ; \mathrm{H}, 2.92 ; \mathrm{N}$, $5.64 ; \mathrm{S}, 5.27$.

f) Model complex $\mathbf{C 6}=[\mathrm{Cu}(\mathbf{2})(\mathbf{6})]^{+}$

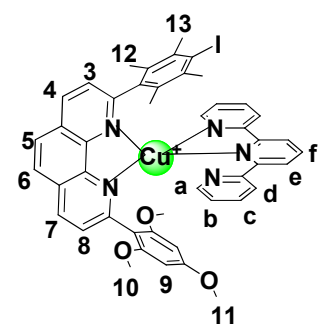

In an NMR tube, phenanthroline $2(0.469 \mathrm{mg}, 0.776 \mu \mathrm{mol})$, terpyridine $6(0.181 \mathrm{mg}, 0.776 \mu \mathrm{mol})$, and $\left.\left[\mathrm{Cu}\left(\mathrm{CH}_{3} \mathrm{CN}\right)_{4}\right)\right] \mathrm{PF}_{6}(0.289 \mathrm{mg}, 0.776 \mu \mathrm{mol})$ were dissolved in 500 of $\mu \mathrm{L} \mathrm{CD}_{2} \mathrm{Cl}_{2}$. NMR spectra were recorded showing quantitative formation of the copper(I) HETTAP complex. Melting point: $150{ }^{\circ} \mathrm{C}$. IR (KBr): $\tilde{v}=558,610,651,768,843,949,992,1031,1067,1128,1158,1184,1206$, 1228, 1338, 1384, 1429, 1456, 1486, 1585, 1608, $2931 \mathrm{~cm}^{-1}$. ${ }^{\mathbf{1}} \mathbf{H}$ NMR (400 MHz, $\left.\mathbf{C D}_{2} \mathbf{C l}_{2}\right): \delta=$ 1.39 (s, 6H, 13-H), 1.93 (s, 6H, 12-H), 3.21 (s, 6H, 10-H), 3.56 (s, 3H, 11-H), 5.65 (s, 2H, 9-H), $7.08\left(\mathrm{ddd},{ }^{3} J=7.4 \mathrm{~Hz},{ }^{3} J=5.6 \mathrm{~Hz},{ }^{4} J=1.6 \mathrm{~Hz}, 2 \mathrm{H}, \mathrm{b}-\mathrm{H}\right), 7.32\left(\mathrm{td},{ }^{3} J=7.4 \mathrm{~Hz},{ }^{4} J=1.6 \mathrm{~Hz}, 2 \mathrm{H}, \mathrm{c}-\right.$ H), $7.62\left(\mathrm{~d},{ }^{3} J=8.4 \mathrm{~Hz}, 1 \mathrm{H}, 8-\mathrm{H}\right), 7.90\left(\mathrm{~d},{ }^{3} J=8.4 \mathrm{~Hz}, 1 \mathrm{H}, 3-\mathrm{H}\right), 8.01$ (ddd, ${ }^{3} J=5.6 \mathrm{~Hz},{ }^{4} J=1.6$ $\left.\mathrm{Hz},{ }^{5} J=0.8 \mathrm{~Hz}, 2 \mathrm{H}, \mathrm{a}-\mathrm{H}\right), 8.04-8.18$ (m, 7H, d-, e-, f-, 5- \& 6-H), 8.49 (d, $\left.{ }^{3} J=8.4 \mathrm{~Hz}, 1 \mathrm{H}, 7-\mathrm{H}\right)$, $8.62\left(\mathrm{~d},{ }^{3} J=8.4 \mathrm{~Hz}, 1 \mathrm{H}, 4-\mathrm{H}\right) \mathrm{ppm} .{ }^{13} \mathbf{C}$ NMR (400 MHz, $\left.\mathbf{C D}_{2} \mathbf{C l}_{2}\right): \delta=19.5,27.2,55.4,55.7$, $90.0,110.0,112.4,121.5,122.9,124.8,126.7,126.7,127.2,127.8,128.5,129.5,131.9,136.5$, 136.7, 137.0, 137.3, 137.9, 140.6, 143.8, 143.9, 148.6, 152.7, 152.8, 155.2, 158.6, 158.9, 162.4. ESI-MS: $\quad m / z \quad(\%) \quad 900.1 \quad(100) \quad[$ [C6]. Elemental analysis: Calculated for $\mathrm{C}_{46} \mathrm{H}_{40} \mathrm{CuF}_{6} \mathrm{IN}_{5} \mathrm{O}_{3} \mathrm{P} \bullet 0.5 \mathrm{CH}_{2} \mathrm{Cl}_{2}:$ C, 51.30; H, 3.80; N, 6.43. Found: C, 51.29; H, 3.63; N, 6.35. 


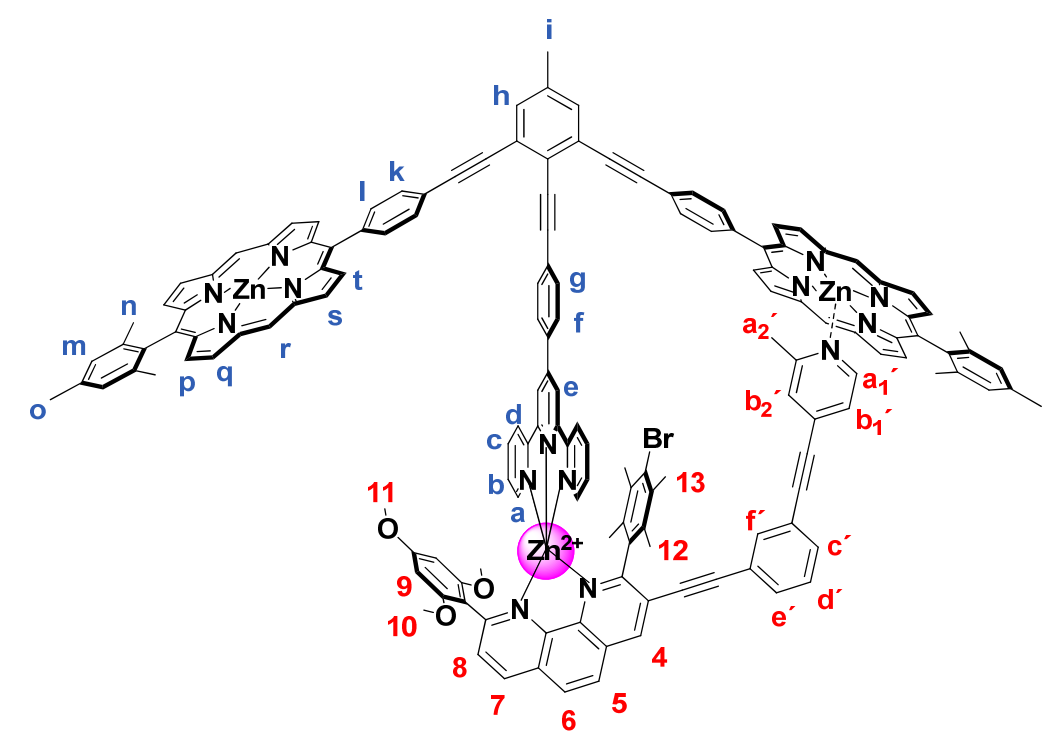

In an NMR tube, stator $\mathbf{S}(0.662 \mathrm{mg}, 0.413 \mu \mathrm{mol})$ and rotator R1 $(0.319 \mathrm{mg}, 0.413 \mu \mathrm{mol})$ were dissolved in $500 \mu \mathrm{L}$ of $\mathrm{CD}_{2} \mathrm{Cl}_{2}$. After addition of $\mathrm{Zn}(\mathrm{OTf})_{2}(0.150 \mathrm{mg}, 0.413 \mu \mathrm{mol})$ as a standard solution in $\mathrm{CD}_{3} \mathrm{CN}$, NMR spectra were measured immediately. Yield: quantitative. Melting point: $>250{ }^{\circ} \mathrm{C}$. IR (KBr): $\tilde{v}=535,571,638,712,724,737,781,817,1026,1031,1056,1227,1262$, 1340, 1397, 1465, 1451, 1510, 1517, 1613, 1653, 2013, 2208, 2854, $2937 \mathrm{~cm}^{-1}$. ${ }^{1} \mathbf{H}$ NMR (600 MHz, $\left.\mathbf{C D}_{2} \mathbf{C l}_{2}\right): \delta=-1.80\left(\mathrm{~s}, 3 \mathrm{H}, \mathrm{a}_{2}{ }^{\prime}-\mathrm{H}\right), 0.70(\mathrm{~s}, 6 \mathrm{H}, 13-\mathrm{H}), 1.59(\mathrm{~s}, 6 \mathrm{H}, 12-\mathrm{H}), 1.68$ (merged with $\left.\mathrm{H}_{2} \mathrm{O}, 1 \mathrm{H}, \mathrm{a}_{1}{ }^{\prime}-\mathrm{H}\right), 1.79$ (s, 12H, n-H), 2.55 (s, 3H, i-H), 2.59 (s, 6H, o-H), 2.61 (s, 3H, 11-H), $2.64(\mathrm{~s}, 6 \mathrm{H}, 10-\mathrm{H}), 5.03(\mathrm{~s}, 2 \mathrm{H}, 9-\mathrm{H}), 5.22\left(\mathrm{dd},{ }^{3} \mathrm{~J}=5.6 \mathrm{~Hz},{ }^{4} J=1.2 \mathrm{~Hz}, 1 \mathrm{H}, \mathrm{b}_{1}{ }^{\prime}-\mathrm{H}\right), 5.31$ (merged with $\left.\mathrm{CDHCl}_{2}, 1 \mathrm{H}, \mathrm{b}_{2}{ }^{\prime}-\mathrm{H}\right), 6.00\left(\mathrm{~s}, 1 \mathrm{H}, \mathrm{f}^{\prime}-\mathrm{H}\right), 6.99$ (ddd, ${ }^{3} \mathrm{~J}=7.8 \mathrm{~Hz},{ }^{4} \mathrm{~J}=1.4 \mathrm{~Hz},{ }^{4} \mathrm{~J}=1.2 \mathrm{~Hz}, 1 \mathrm{H}$, $\left.\mathrm{e}^{\prime} / \mathrm{c}^{\prime}-\mathrm{H}\right), 7.07\left(\mathrm{t},{ }^{3} J=7.8 \mathrm{~Hz}, 1 \mathrm{H}, \mathrm{d}^{\prime}-\mathrm{H}\right), 7.22\left(\mathrm{ddd},{ }^{3} J=7.8 \mathrm{~Hz},{ }^{4} J=1.4 \mathrm{~Hz},{ }^{4} J=1.2 \mathrm{~Hz}, 1 \mathrm{H}, \mathrm{c}^{\prime} / \mathrm{e}^{\prime}-\right.$ H), 7.32 (s, 4H, m-H), 7.38 (ddd, $\left.{ }^{3} J=8.0 \mathrm{~Hz},{ }^{3} J=5.2 \mathrm{~Hz},{ }^{4} J=1.2 \mathrm{~Hz}, 2 \mathrm{H}, \mathrm{b}-\mathrm{H}\right), 7.50$ (dd, ${ }^{3} J=5.2$ $\left.\mathrm{Hz},{ }^{4} J=1.2 \mathrm{~Hz}, 2 \mathrm{H}, \mathrm{a}-\mathrm{H}\right), 7.73(\mathrm{~s}, 2 \mathrm{H}, \mathrm{h}-\mathrm{H}), 7.85$ (d, $\left.{ }^{3} J=8.4 \mathrm{~Hz}, 1 \mathrm{H}, 8-\mathrm{H}\right), 8.11\left(\mathrm{td},{ }^{3} J=8.0 \mathrm{~Hz}\right.$, $\left.{ }^{4} J=1.2 \mathrm{~Hz}, 2 \mathrm{H}, \mathrm{c}-\mathrm{H}\right), 8.13\left(\mathrm{~d},{ }^{3} J=8.0 \mathrm{~Hz}, 4 \mathrm{H}, \mathrm{k}-\mathrm{H}\right), 8.13$ (d, merged with k-H, 2H, f/g-H), 8.22 (br, $2 \mathrm{H}, \mathrm{g} / \mathrm{f}-\mathrm{H}), 8.29$ (d, $\left.{ }^{3} J=8.8 \mathrm{~Hz}, 1 \mathrm{H}, 6 / 5-\mathrm{H}\right), 8.34$ (d, $\left.{ }^{3} J=8.0 \mathrm{~Hz}, 4 \mathrm{H}, 1-\mathrm{H}\right), 8.37$ (d, ${ }^{3} J=8.8 \mathrm{~Hz}$, $1 \mathrm{H}, 5 / 6-\mathrm{H}), 8.49\left(\mathrm{dd},{ }^{3} J=8.0 \mathrm{~Hz},{ }^{4} \mathrm{~J}=1.2 \mathrm{~Hz}, 2 \mathrm{H}, \mathrm{d}-\mathrm{H}\right), 8.50(\mathrm{~s}, 2 \mathrm{H}, \mathrm{e}-\mathrm{H}), 8.84\left(\mathrm{~d},{ }^{3} J=8.4 \mathrm{~Hz}, 1 \mathrm{H}\right.$, 7-H),8.86 (s, 1H, 4-H), 8.88 (d, $\left.{ }^{3} J=4.4 \mathrm{~Hz}, 4 \mathrm{H}, \mathrm{p}-\mathrm{H}\right), 9.15\left(\mathrm{~d},{ }^{3} J=4.4 \mathrm{~Hz}, 4 \mathrm{H}, \mathrm{t}-\mathrm{H}\right), 9.35\left(\mathrm{~d},{ }^{3} J=\right.$ $4.4 \mathrm{~Hz}, 4 \mathrm{H}, \mathrm{q}-\mathrm{H}), 9.45$ (d, $\left.{ }^{3} J=4.4 \mathrm{~Hz}, 4 \mathrm{H}, \mathrm{s}-\mathrm{H}\right), 10.22$ (s, 4H, r-H) ppm. ESI-MS: $m / z(\%) 1221.3$ (100) $\left[[\mathrm{Zn}(\mathbf{S})(\mathbf{R 1})]^{2+}\right]$. Elemental analysis: Calculated for $\mathrm{C}_{153} \mathrm{H}_{107} \mathrm{BrF}_{6} \mathrm{~N}_{14} \mathrm{O}_{9} \mathrm{~S}_{2} \mathrm{Zn}_{3} \cdot \mathrm{CH}_{2} \mathrm{Cl}_{2} \cdot \mathrm{H}_{2} \mathrm{O}$ : C, 65.06; H, 3.94; N, 6.90; S, 2.26. Found: C, 65.01; H, 3.69; N, 6.57; S, 1.98. 


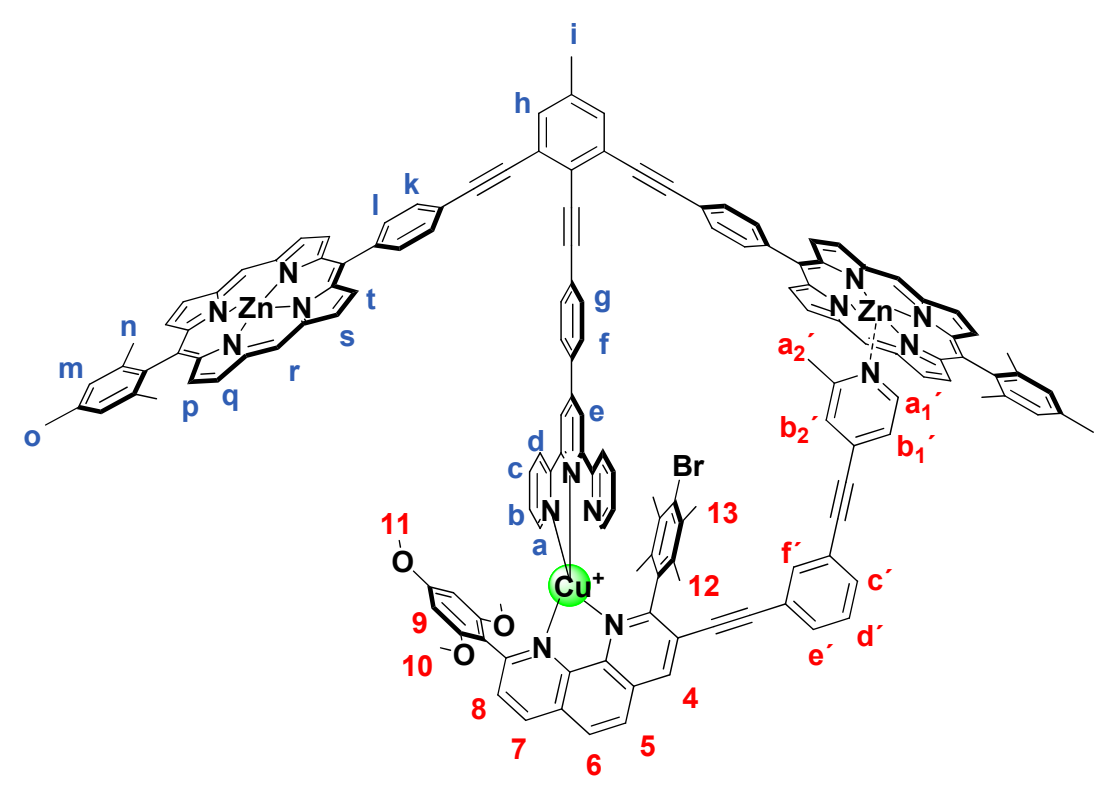

In an NMR tube, stator $\mathbf{S}(0.680 \mathrm{mg}, 0.424 \mu \mathrm{mol})$, rotator $\mathbf{R} 1(0.327 \mathrm{mg}, 0.424 \mu \mathrm{mol})$ and $\left.\left[\mathrm{Cu}\left(\mathrm{CH}_{3} \mathrm{CN}\right)_{4}\right)\right] \mathrm{PF}_{6}(0.159 \mathrm{mg}, 0.424 \mu \mathrm{mol})$ were dissolved in $500 \mu \mathrm{L}$ of $\mathrm{CD}_{2} \mathrm{Cl}_{2}$ and ${ }^{1} \mathrm{H} \mathrm{NMR}$ spectra were measured immediately. NMR and ESI-MS spectra confirm the quantitative formation of nanorotor $[\mathrm{Cu}(\mathbf{S})(\mathbf{R 1})]^{+}$. Melting point $>250{ }^{\circ} \mathrm{C}$. IR $(\mathbf{K B r}): \tilde{v}=558,683,702,720,734,786$, 812, 845, 994, 1057, 1129, 1158, 1206, 1227, 1283, 1339, 1393, 1455, 1519, 1548, 1605, 2212, 2852, $2922 \mathrm{~cm}^{-1} .{ }^{1} \mathbf{H}$ NMR (600 MHz, $\left.\mathbf{C D}_{2} \mathbf{C l}_{2}\right): \delta=-1.79\left(\mathrm{~s}, 3 \mathrm{H}, \mathrm{a}_{2}{ }^{\prime}-\mathrm{H}\right), 1.08(\mathrm{~s}, 6 \mathrm{H}, 13-\mathrm{H}), 1.54$ (s, 6H, 12-H), 1.69 (d, $\left.{ }^{3} J=6.4 \mathrm{~Hz}, 1 \mathrm{H}, \mathrm{a}_{1}{ }^{\prime}-\mathrm{H}\right), 1.80$ (s, 12H, n-H), 2.61 (s, 3H, i-H), 2.66 (s, 6H, o$\mathrm{H}), 2.84(\mathrm{~s}, 6 \mathrm{H}, 10-\mathrm{H}), 2.90(\mathrm{~s}, 3 \mathrm{H}, 11-\mathrm{H}), 5.16(\mathrm{~s}, 2 \mathrm{H}, 9-\mathrm{H}), 5.27$ (merged with $\mathrm{CDHCl}_{2}, 1 \mathrm{H}, \mathrm{b}_{1}{ }^{\prime}-$ $\mathrm{H}), 5.41$ (brs, $\left.1 \mathrm{H}, \mathrm{b}_{2}{ }^{\prime}-\mathrm{H}\right), 6.04\left(\mathrm{t},{ }^{4} \mathrm{~J}=1.2 \mathrm{~Hz}, 1 \mathrm{H}, \mathrm{f}^{\prime}-\mathrm{H}\right), 6.97\left(\mathrm{ddd},{ }^{3} \mathrm{~J}=7.8 \mathrm{~Hz},{ }^{4} \mathrm{~J}=1.4 \mathrm{~Hz},{ }^{4} \mathrm{~J}=\right.$ $\left.1.2 \mathrm{~Hz}, 1 \mathrm{H}, \mathrm{e}^{\prime} / \mathrm{c}^{\prime}-\mathrm{H}\right), 6.99$ (ddd, $\left.{ }^{3} J=7.8 \mathrm{~Hz},{ }^{3} J=4.8 \mathrm{~Hz},{ }^{4} J=1.2 \mathrm{~Hz}, 2 \mathrm{H}, \mathrm{b}-\mathrm{H}\right), 7.05\left(\mathrm{t},{ }^{3} J=7.8 \mathrm{~Hz}\right.$, $\left.1 \mathrm{H}, \mathrm{d}^{\prime}-\mathrm{H}\right), 7.17\left(\mathrm{ddd},{ }^{3} J=7.8 \mathrm{~Hz},{ }^{4} J=1.4 \mathrm{~Hz},{ }^{4} J=1.2 \mathrm{~Hz}, 1 \mathrm{H}, \mathrm{c}^{\prime} / \mathrm{e}^{\prime}-\mathrm{H}\right), 7.26\left(\mathrm{td},{ }^{3} J=7.8 \mathrm{~Hz},{ }^{4} J=\right.$ $1.2 \mathrm{~Hz}, 2 \mathrm{H}, \mathrm{c}-\mathrm{H}), 7.34$ (s, 4H, m-H), $7.71\left(\mathrm{~d},{ }^{3} J=8.8 \mathrm{~Hz}, 1 \mathrm{H}, 8-\mathrm{H}\right), 7.73$ (s, 2H, h-H), $7.86\left(\mathrm{dd},{ }^{3} J=\right.$ $\left.4.8 \mathrm{~Hz},{ }^{4} J=1.2 \mathrm{~Hz}, 2 \mathrm{H}, \mathrm{a}-\mathrm{H}\right), 7.96\left(\mathrm{~d},{ }^{3} J=8.8 \mathrm{~Hz}, 1 \mathrm{H}, 5 / 6-\mathrm{H}\right), 7.97$ (d, $\left.{ }^{3} J=8.0 \mathrm{~Hz}, 2 \mathrm{H}, \mathrm{g} / \mathrm{f}-\mathrm{H}\right)$, $8.01\left(\mathrm{dd},{ }^{3} J=7.8 \mathrm{~Hz},{ }^{4} \mathrm{~J}=1.2 \mathrm{~Hz}, 2 \mathrm{H}, \mathrm{d}-\mathrm{H}\right), 8.10\left(\mathrm{~d},{ }^{3} J=8.8 \mathrm{~Hz}, 1 \mathrm{H}, 6 / 5-\mathrm{H}\right), 8.15\left(\mathrm{~d},{ }^{3} J=8.0 \mathrm{~Hz}\right.$, $4 \mathrm{H}, \mathrm{k}-\mathrm{H}), 8.18$ (d, $\left.{ }^{3} J=8.0 \mathrm{~Hz}, 2 \mathrm{H}, \mathrm{f} / \mathrm{g}-\mathrm{H}\right), 8.26$ (s, 2H, e-H), 8.35 (d, $\left.{ }^{3} J=8.0 \mathrm{~Hz}, 2 \mathrm{H}, 1-\mathrm{H}\right), 8.48$ (s, $1 \mathrm{H}, 4-\mathrm{H}), 8.50\left(\mathrm{~d},{ }^{3} J=8.8 \mathrm{~Hz}, 1 \mathrm{H}, 7-\mathrm{H}\right), 8.92\left(\mathrm{~d},{ }^{3} J=4.4 \mathrm{~Hz}, 4 \mathrm{H}, \mathrm{p}-\mathrm{H}\right), 9.17\left(\mathrm{~d},{ }^{3} J=4.4 \mathrm{~Hz}, 4 \mathrm{H}, \mathrm{t}-\right.$ H), 9.38 (d, $\left.{ }^{3} J=4.4 \mathrm{~Hz}, 4 \mathrm{H}, \mathrm{q}-\mathrm{H}\right), 9.46$ (d, $\left.{ }^{3} J=4.4 \mathrm{~Hz}, 4 \mathrm{H}, \mathrm{s}-\mathrm{H}\right), 10.24$ (s, 4H, r-H) ppm. ESI-MS: $m / z \quad(\%) \quad 2470.7 \quad(100) \quad\left[\mathrm{Cu}(\mathbf{S})(\mathbf{R 1})\left(\mathrm{CH}_{3} \mathrm{OH}\right)\right]^{+}$. Elemental analysis: Calculated for $\mathrm{C}_{151} \mathrm{H}_{107} \mathrm{BrCuF}_{6} \mathrm{~N}_{14} \mathrm{O}_{3} \mathrm{PZn}_{2} \cdot 3 \mathrm{H}_{2} \mathrm{O}: \mathrm{C}, 68.73 ; \mathrm{H}, 4.32 ; \mathrm{N}, 7.43$. Found: C, 68.80; H, 4.32; N, 7.11. 


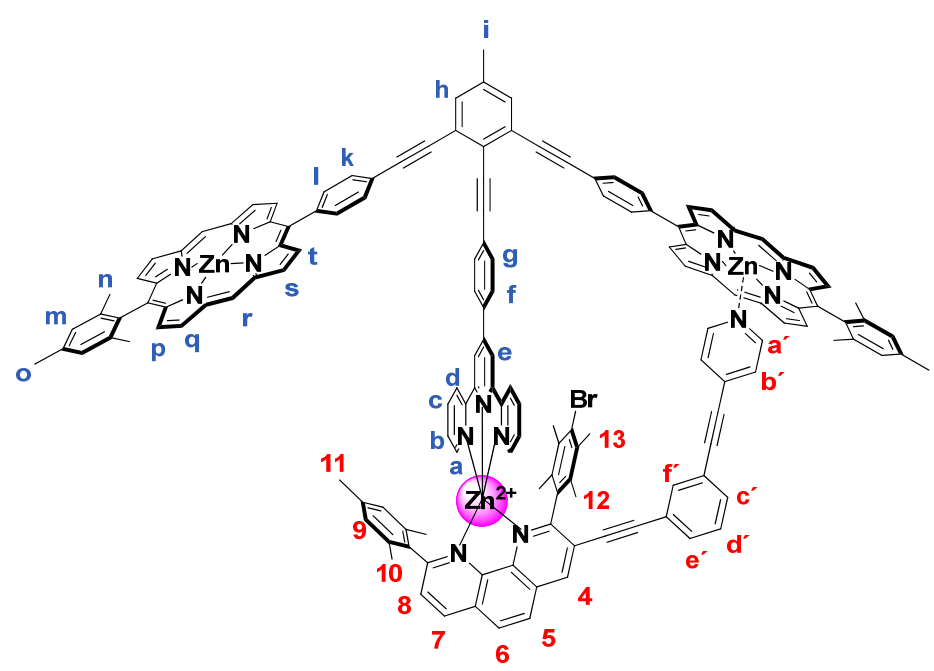

In an NMR tube, stator $\mathbf{S}(0.656 \mathrm{mg}, 0.409 \mu \mathrm{mol})$ and rotator R1 $(0.291 \mathrm{mg}, 0.409 \mu \mathrm{mol})$ were dissolved in $500 \mu \mathrm{L}$ of $\mathrm{CD}_{2} \mathrm{Cl}_{2}$. After addition of $\mathrm{Zn}(\mathrm{OTf})_{2}(0.149 \mathrm{mg}, 0.409 \mu \mathrm{mol})$ as a standard solution in $\mathrm{CD}_{3} \mathrm{CN}$, NMR spectra were measured immediately. Yield: quantitative. Melting point: $>250{ }^{\circ} \mathrm{C}$. IR (KBr): $\tilde{v}=518,573,638,703,722,734,785,812,832,852,994,1015,1030,1057$, 1161, 1224, 1259, 1393, 1429, 1477, 1519, 1546, 1603, 2213, $2920 \mathrm{~cm}^{-1}$. ${ }^{\mathbf{1}} \mathbf{H}$ NMR (600 MHz, $\left.\mathbf{C D}_{2} \mathbf{C l}_{2}\right): \delta=0.80(\mathrm{~s}, 6 \mathrm{H}, 13-\mathrm{H}), 0.89(\mathrm{~s}, 6 \mathrm{H}, 10-\mathrm{H}), 1.07$ (s, 3H, 11-H), $1.48(\mathrm{~s}, 6 \mathrm{H}, 12-\mathrm{H}), 1.78(\mathrm{~s}$, $12 \mathrm{H}, \mathrm{n}-\mathrm{H}), 2.19\left(\mathrm{~d},{ }^{3} J=6.4 \mathrm{~Hz}, 2 \mathrm{H}, \mathrm{a}^{\prime}-\mathrm{H}\right), 2.60(\mathrm{~s}, 3 \mathrm{H}, \mathrm{i}-\mathrm{H}), 2.63(\mathrm{~s}, 6 \mathrm{H}, \mathrm{o}-\mathrm{H}), 5.33\left(\mathrm{~d},{ }^{3} J=6.4 \mathrm{~Hz}\right.$, $\left.2 \mathrm{H}, \mathrm{b}^{\prime}-\mathrm{H}\right), 5.63(\mathrm{~s}, 2 \mathrm{H}, 9-\mathrm{H}), 5.85\left(\mathrm{~s}, 1 \mathrm{H}, \mathrm{f}^{\prime}-\mathrm{H}\right), 6.96\left(\mathrm{ddd},{ }^{3} J=7.8 \mathrm{~Hz},{ }^{4} J=1.4 \mathrm{~Hz},{ }^{4} J=1.2 \mathrm{~Hz}\right.$, $\left.1 \mathrm{H}, \mathrm{e}^{\prime} / \mathrm{c}^{\prime}-\mathrm{H}\right), 7.06\left(\mathrm{t},{ }^{3} J=7.8 \mathrm{~Hz}, 1 \mathrm{H}, \mathrm{d}^{\prime}-\mathrm{H}\right), 7.23\left(\mathrm{ddd},{ }^{3} J=7.8 \mathrm{~Hz},{ }^{4} J=1.4 \mathrm{~Hz},{ }^{4} \mathrm{~J}=1.2 \mathrm{~Hz}, 1 \mathrm{H}\right.$, $\left.\mathrm{c}^{\prime} / \mathrm{e}^{\prime}-\mathrm{H}\right), 7.32$ (s, 4H, m-H), 7.45 (ddd, $\left.{ }^{3} J=7.8 \mathrm{~Hz},{ }^{3} J=5.2 \mathrm{~Hz},{ }^{4} J=1.2 \mathrm{~Hz}, 2 \mathrm{H}, \mathrm{b}-\mathrm{H}\right), 7.69$ (dd, ${ }^{3} J$ $\left.=5.2 \mathrm{~Hz},{ }^{4} J=1.2 \mathrm{~Hz}, 2 \mathrm{H}, \mathrm{a}-\mathrm{H}\right), 7.73(\mathrm{~s}, 2 \mathrm{H}, \mathrm{h}-\mathrm{H}), 7.86\left(\mathrm{~d},{ }^{3} J=8.4 \mathrm{~Hz}, 1 \mathrm{H}, 8-\mathrm{H}\right), 8.09\left(\mathrm{~d},{ }^{3} J=8.0\right.$ $\mathrm{Hz}, 2 \mathrm{H}, \mathrm{g} / \mathrm{f}-\mathrm{H}), 8.14$ (d, $\left.{ }^{3} J=8.0 \mathrm{~Hz}, 4 \mathrm{H}, \mathrm{k}-\mathrm{H}\right), 8.17$ (td, $\left.{ }^{3} J=7.8 \mathrm{~Hz},{ }^{4} J=1.2 \mathrm{~Hz}, 2 \mathrm{H}, \mathrm{c}-\mathrm{H}\right), 8.25$ (d, $\left.{ }^{3} J=8.0 \mathrm{~Hz}, 2 \mathrm{H}, \mathrm{f} / \mathrm{g}-\mathrm{H}\right), 8.35\left(\mathrm{~d},{ }^{3} J=8.8 \mathrm{~Hz}, 1 \mathrm{H}, 5 / 6-\mathrm{H}\right), 8.37\left(\mathrm{~d},{ }^{3} J=8.0 \mathrm{~Hz}, 4 \mathrm{H}, 1-\mathrm{H}\right), 8.39\left(\mathrm{~d},{ }^{3} J=\right.$ $8.8 \mathrm{~Hz}, 1 \mathrm{H}, 6 / 5-\mathrm{H}), 8.45$ (s, 2H, e-H), $8.47\left(\mathrm{~d},{ }^{3} J=7.8 \mathrm{~Hz},{ }^{4} J=1.2 \mathrm{~Hz},{ }^{5} J=0.8 \mathrm{~Hz}, 2 \mathrm{H}, \mathrm{d}-\mathrm{H}\right), 8.87$ $\left(\mathrm{d},{ }^{3} J=4.4 \mathrm{~Hz}, 4 \mathrm{H}, \mathrm{p}-\mathrm{H}\right), 8.90$ (s, 1H, 4-H), 8.94 (d, $\left.{ }^{3} J=8.4 \mathrm{~Hz}, 1 \mathrm{H}, 7-\mathrm{H}\right), 9.16\left(\mathrm{~d},{ }^{3} J=4.4 \mathrm{~Hz}, 4 \mathrm{H}\right.$, t-H), $9.34\left(\mathrm{~d},{ }^{3} J=4.4 \mathrm{~Hz}, 4 \mathrm{H}, \mathrm{q}-\mathrm{H}\right), 9.46\left(\mathrm{~d},{ }^{3} J=4.4 \mathrm{~Hz}, 4 \mathrm{H}, \mathrm{s}-\mathrm{H}\right), 10.21$ (s, 4H, r-H) ppm. ESIMS: $m / z \quad(\%) \quad 1189.8 \quad(100) \quad\left[[\mathrm{Zn}(\mathbf{S})(\mathbf{R 2})]^{2+}\right]$. Elemental analysis: Calculated for $\mathrm{C}_{152} \mathrm{H}_{105} \mathrm{BrF}_{6} \mathrm{~N}_{14} \mathrm{O}_{6} \mathrm{~S}_{2} \mathrm{Zn}_{3} \cdot \mathrm{CH}_{2} \mathrm{Cl}_{2} \cdot \mathrm{H}_{2} \mathrm{O}: \mathrm{C}, 66.08 ; \mathrm{H}, 3.95 ; \mathrm{N}, 7.05 ; \mathrm{S}, 2.31$. Found: C, 65.83; H, $3.86 ; \mathrm{N}, 6.74 ; \mathrm{S}, 2.24$. 


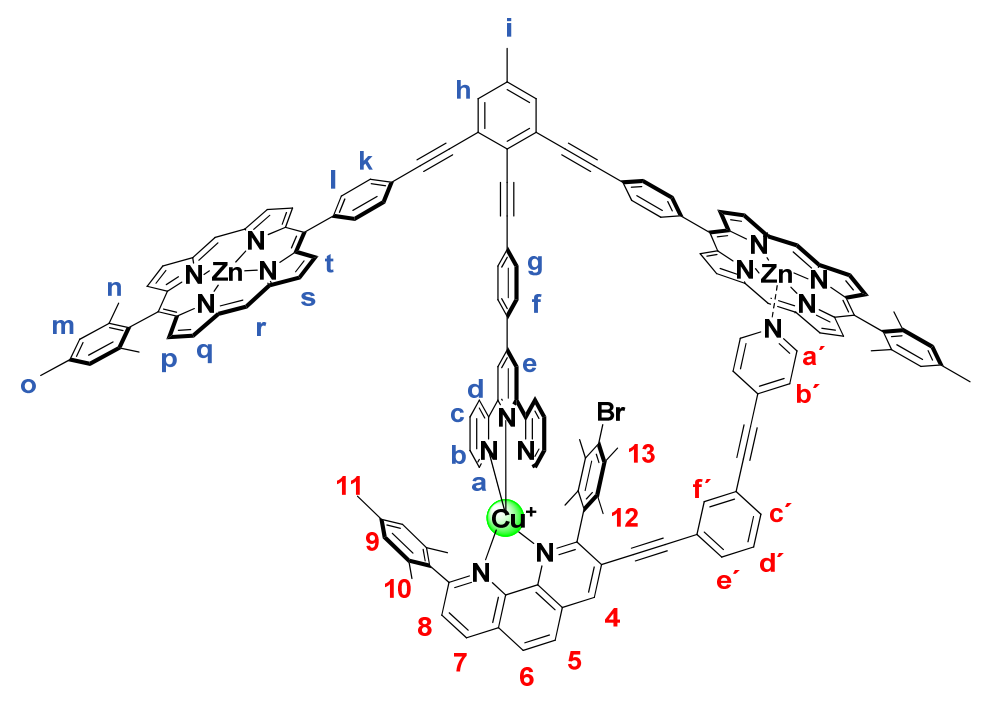

In an NMR tube, stator $\mathbf{S}(0.664 \mathrm{mg}, 0.414 \mu \mathrm{mol})$, rotator $\mathbf{R 2}(0.294 \mathrm{mg}, 0.414 \mu \mathrm{mol})$ and $\left.\left[\mathrm{Cu}\left(\mathrm{CH}_{3} \mathrm{CN}\right)_{4}\right)\right] \mathrm{PF}_{6}(0.154 \mathrm{mg}, 0.414 \mu \mathrm{mol})$ were dissolved in $500 \mu \mathrm{L}$ of $\mathrm{CD}_{2} \mathrm{Cl}_{2}$. NMR and ESIMS spectra confirmed the quantitative formation of nanorotor $[\mathrm{Cu}(\mathbf{S})(\mathbf{R 2})] \mathrm{PF}_{6}$. Melting point > $250{ }^{\circ} \mathrm{C}$. IR (KBr): $\tilde{v}=474,523,753,662,714,726,746,787,819,834,1022,1039,1159,1236$, 1346, 1384, 1436, 1538, 1605, 1687, 2216, 2931, $3012 \mathrm{~cm}^{-1} .{ }^{\mathbf{1}} \mathbf{H}$ NMR (600 MHz, $\left.\mathbf{C D}_{2} \mathbf{C l}_{2}\right): \delta=$ $1.21(\mathrm{~s}, 6 \mathrm{H}, 13-\mathrm{H}), 1.29(\mathrm{~s}, 6 \mathrm{H}, 10-\mathrm{H}), 1.53(\mathrm{~s}, 9 \mathrm{H}, 11+12-\mathrm{H}), 1.81(\mathrm{~s}, 12 \mathrm{H}, \mathrm{n}-\mathrm{H}), 2.19\left(\mathrm{~d},{ }^{3} J=6.6\right.$ $\left.\mathrm{Hz}, 2 \mathrm{H}, \mathrm{a}^{\prime}-\mathrm{H}\right), 2.61$ (s, 3H, i-H), $2.66(\mathrm{~s}, 6 \mathrm{H}, \mathrm{o}-\mathrm{H}), 5.40\left(\mathrm{~d},{ }^{3} \mathrm{~J}=6.6 \mathrm{~Hz}, 2 \mathrm{H}, \mathrm{b}^{\prime}-\mathrm{H}\right), 5.97\left(\mathrm{t},{ }^{4} J=1.2\right.$ $\left.\mathrm{Hz}, 1 \mathrm{H}, \mathrm{f}^{\prime}-\mathrm{H}\right), 6.03$ (s, 2H, 9-H), $6.94\left(\mathrm{ddd},{ }^{3} J=7.8 \mathrm{~Hz},{ }^{4} J=1.4 \mathrm{~Hz},{ }^{4} J=1.2 \mathrm{~Hz}, 1 \mathrm{H}, \mathrm{e}^{\prime} / \mathrm{c}^{\prime}-\mathrm{H}\right), 6.96$ (ddd, $\left.{ }^{3} J=7.8 \mathrm{~Hz},{ }^{3} J=4.8 \mathrm{~Hz},{ }^{4} J=1.2 \mathrm{~Hz}, 2 \mathrm{H}, \mathrm{b}-\mathrm{H}\right), 7.03\left(\mathrm{t},{ }^{3} J=7.8 \mathrm{~Hz}, 1 \mathrm{H}, \mathrm{d}-\mathrm{H}\right), 7.19$ (ddd, ${ }^{3} J=$ $\left.7.8 \mathrm{~Hz},{ }^{4} \mathrm{~J}=1.4 \mathrm{~Hz},{ }^{4} \mathrm{~J}=1.2 \mathrm{~Hz}, 1 \mathrm{H}, \mathrm{c}^{\prime} / \mathrm{e}^{\prime}-\mathrm{H}\right), 7.34(\mathrm{~s}, 4 \mathrm{H}, \mathrm{m}-\mathrm{H}), 7.42\left(\mathrm{td},{ }^{3} J=7.8 \mathrm{~Hz},{ }^{4} \mathrm{~J}=1.2 \mathrm{~Hz}\right.$, $4 \mathrm{H}, \mathrm{c}-\mathrm{H}), 7.44$ (ddd, $\left.{ }^{3} J=4.8 \mathrm{~Hz},{ }^{4} J=1.2 \mathrm{~Hz},{ }^{5} J=0.8 \mathrm{~Hz}, 2 \mathrm{H}, \mathrm{a}-\mathrm{H}\right), 7.64\left(\mathrm{~d},{ }^{3} J=8.8 \mathrm{~Hz}, 1 \mathrm{H}, 8-\mathrm{H}\right)$, 7.74 (s, 2H, h-H), 7.78 (ddd, $\left.{ }^{3} J=7.8 \mathrm{~Hz},{ }^{4} J=1.2 \mathrm{~Hz},{ }^{5} J=0.8 \mathrm{~Hz}, 2 \mathrm{H}, \mathrm{d}-\mathrm{H}\right), 7.97\left(\mathrm{~d},{ }^{3} J=8.0 \mathrm{~Hz}\right.$, $2 \mathrm{H}, \mathrm{g} / \mathrm{f}-\mathrm{H}), 8.07$ (d, $\left.{ }^{3} J=8.8 \mathrm{~Hz}, 1 \mathrm{H}, 5 / 6-\mathrm{H}\right), 8.14$ (s, 2H, e-H), 8.16 (d, $\left.{ }^{3} J=8.0 \mathrm{~Hz}, 4 \mathrm{H}, \mathrm{k}-\mathrm{H}\right), 8.16$ $\left(\mathrm{d},{ }^{3} J=8.0 \mathrm{~Hz}, 2 \mathrm{H}, \mathrm{f} / \mathrm{g}-\mathrm{H}\right), 8.19$ (d, $\left.{ }^{3} J=8.8 \mathrm{~Hz}, 1 \mathrm{H}, 6 / 5-\mathrm{H}\right), 8.39\left(\mathrm{~d},{ }^{3} J=8.0 \mathrm{~Hz}, 4 \mathrm{H}, 1-\mathrm{H}\right), 8.54$ (d, $\left.{ }^{3} J=8.8 \mathrm{~Hz}, 1 \mathrm{H}, 7-\mathrm{H}\right), 8.56(\mathrm{~s}, 1 \mathrm{H}, 4-\mathrm{H}), 8.92\left(\mathrm{~d},{ }^{3} J=4.4 \mathrm{~Hz}, 4 \mathrm{H}, \mathrm{p}-\mathrm{H}\right), 9.19\left(\mathrm{~d},{ }^{3} J=4.4 \mathrm{~Hz}, 4 \mathrm{H}, \mathrm{t}-\right.$ H), 9.37 (d, $\left.{ }^{3} J=4.4 \mathrm{~Hz}, 4 \mathrm{H}, \mathrm{q}-\mathrm{H}\right), 9.47$ (d, $\left.{ }^{3} J=4.4 \mathrm{~Hz}, 4 \mathrm{H}, \mathrm{s}-\mathrm{H}\right), 10.24$ (s, 4H, r-H) ppm. ESI-MS: $m / z \quad(\%) \quad 2409.8 \quad(100) \quad\left[\mathrm{Cu}(\mathbf{S})(\mathbf{R 2})\left(\mathrm{CH}_{3} \mathrm{OH}\right)\left(\mathrm{H}_{2} \mathrm{O}\right)\right]^{+}$. Elemental analysis: Calculated for $\mathrm{C}_{150} \mathrm{H}_{105} \mathrm{BrCuF}_{6} \mathrm{~N}_{14} \mathrm{PZn}_{2} \cdot \mathrm{CH}_{2} \mathrm{Cl}_{2} \cdot \mathrm{H}_{2} \mathrm{O}: \mathrm{C}, 69.07 ; \mathrm{H}, 4.18 ; \mathrm{N}, 7.47$. Found: C, 69.03; H, 3.81; N, 7.46 . 
$\mathbf{C 1 1}=\left[\mathrm{Cu}_{2}(\mathbf{R 2})_{2}\right]^{2+}$

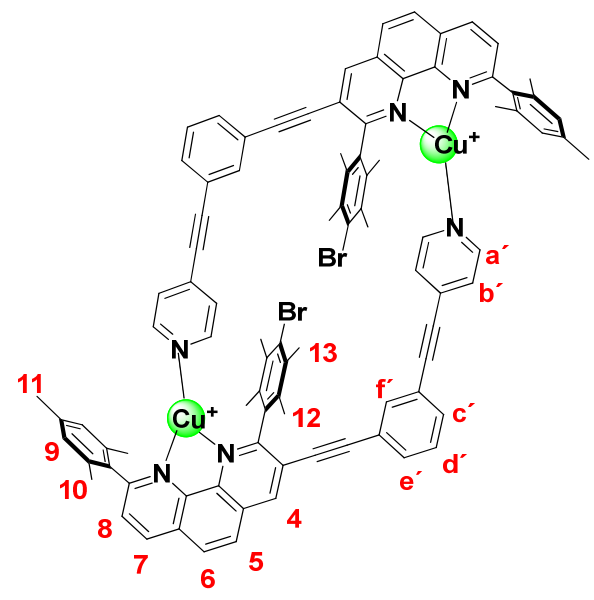

In an NMR tube, rotator $\mathbf{R 2}(0.612 \mathrm{mg}, 0.861 \mu \mathrm{mol})$ and $\left[\mathrm{Cu}\left(\mathrm{CH}_{3} \mathrm{CN}\right)_{4}\right] \mathrm{PF}_{6}(0.320 \mathrm{mg}, 0.861 \mu \mathrm{mol})$ were dissolved in $500 \mu \mathrm{L}$ of $\mathrm{CD}_{2} \mathrm{Cl}_{2}$. ${ }^{1} \mathrm{H} \mathrm{NMR}$ and ESI-MS spectra confirm the quantitative formation of dimer $\left[\mathrm{Cu}_{2}(\mathbf{R 2})_{2}\right]^{2+}$. Melting point: $183{ }^{\circ} \mathrm{C}$. IR $(\mathbf{K B r}): \tilde{v}=558,684,843,948,1016$, 1084, 1128, 1158, 1206, 1227, 1336, 1384, 1417, 1463, 1491, 1610, 2215, $2925 \mathrm{~cm}^{-1}$. ${ }^{\mathbf{1}} \mathbf{H}$ NMR (400 MHz, $\left.\mathbf{C D}_{2} \mathbf{C l}_{2}: \mathbf{C H}_{3} \mathbf{C N}=4: 1\right): \delta=2.01$ (s, 12H, 13-H), 2.07 (s, 12H, 12-H), 2.35 (s, 6H, 11H), 2.51 (s, 12H, 10-H), 6.87 (brs, 4H, a'-H), 6.99 (s, 4H, 9-H), 7.05 (brs, 2H, f'-H), 7.21 (d, ${ }^{3} J=$ $\left.5.6 \mathrm{~Hz}, 4 \mathrm{H}, \mathrm{b}^{\prime}-\mathrm{H}\right), 7.40\left(\mathrm{t},{ }^{3} J=7.8 \mathrm{~Hz}, 2 \mathrm{H}, \mathrm{d}^{\prime}-\mathrm{H}\right), 7.45\left(\mathrm{~d},{ }^{3} J=7.8 \mathrm{~Hz}, 2 \mathrm{H}, \mathrm{c}^{\prime} / \mathrm{e}^{\prime}-\mathrm{H}\right), 7.56\left(\mathrm{~d},{ }^{3} J=\right.$ $\left.7.8 \mathrm{~Hz}, 2 \mathrm{H}, \mathrm{e}^{\prime} / \mathrm{c}^{\prime}-\mathrm{H}\right), 7.98\left(\mathrm{~d},{ }^{3} J=8.6 \mathrm{~Hz}, 2 \mathrm{H}, 8-\mathrm{H}\right), 8.18\left(\mathrm{~d},{ }^{3} J=8.8 \mathrm{~Hz}, 2 \mathrm{H}, 5 / 6-\mathrm{H}\right), 8.22\left(\mathrm{~d},{ }^{3} J=\right.$ $8.8 \mathrm{~Hz}, 2 \mathrm{H}, 6 / 5-\mathrm{H}), 8.74\left(\mathrm{~d},{ }^{3} J=8.6 \mathrm{~Hz}, 2 \mathrm{H}, 7-\mathrm{H}\right), 8.84$ (s, 2H, 4-H) ppm. ESI-MS: $m / z(\%) 774.5$ (100) $\left[\mathrm{Cu}_{2}(\mathbf{R 2})_{2}\right]^{2+}$. Elemental analysis: Calculated for $\mathrm{C}_{92} \mathrm{H}_{72} \mathrm{Br}_{2} \mathrm{Cu}_{2} \mathrm{~F}_{12} \mathrm{~N}_{6} \mathrm{P}_{2}: \mathrm{C}, 55.24 ; \mathrm{H}, 3.90$; N, 4.07. Found: C, 55.23; H, 3.59; N, 3.96.

$\mathbf{C 1 2}=\left[\mathrm{Cu}_{2}(\mathbf{R 1})_{2}\right]^{2+}$

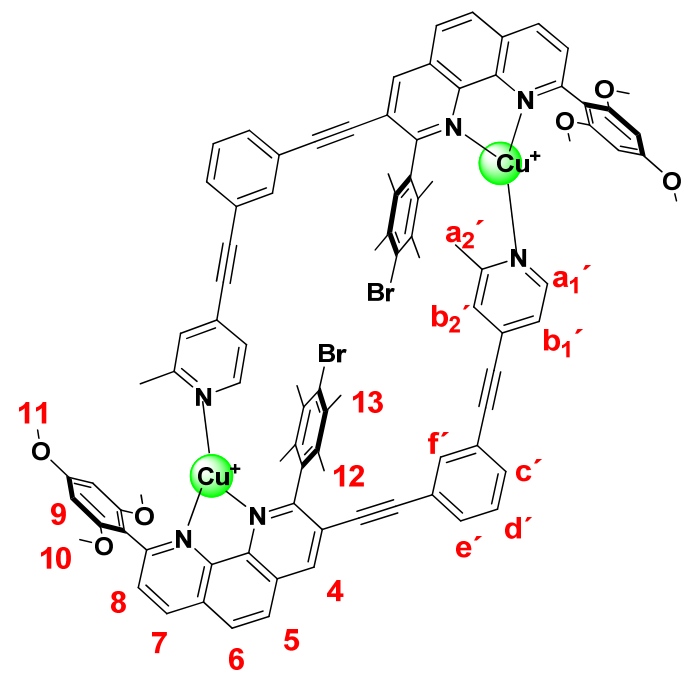


In an NMR tube, rotator $\mathbf{R} 1(0.552 \mathrm{mg}, 0.715 \mu \mathrm{mol})$ and $\left[\mathrm{Cu}\left(\mathrm{CH}_{3} \mathrm{CN}\right)_{4}\right] \mathrm{PF}_{6}(0.267 \mathrm{mg}, 0.715 \mu \mathrm{mol})$ were dissolved in $500 \mu \mathrm{L}$ of $\mathrm{CD}_{2} \mathrm{Cl}_{2}$. ${ }^{1} \mathrm{H}$ NMR and ESI-MS spectra confirm the quantitative formation of dimer $\left[\mathrm{Cu}_{2}(\mathbf{R 1})_{2}\right]^{2+}$. Melting point: $191{ }^{\circ} \mathrm{C}$. IR $(\mathbf{K B r}): \tilde{v}=552,617,725,817,992$, $1031,1113,1189,1215,1263,1347,1473,1509,1592,1613,2220,2531,2857,2923 \mathrm{~cm}^{-1} .{ }^{1} \mathbf{H}$ NMR (400 MHz, $\left.\mathbf{C D}_{2} \mathbf{C l}_{2}\right): \delta=1.97$ (s, 12H, 13-H), 2.04 (s, 6H, a $\left.{ }_{2}^{\prime}-\mathrm{H}\right), 2.33$ (s, 12H, 12-H), 2.67 (s, 12H, 10-H), 2.77 (s, 6H, 11-H), 6.11 (s, 4H, 9-H), 7.11 (brs, 2H, a $\left.{ }^{\prime}-\mathrm{H}\right), 7.14$ (brs, 2H, f'-H), 7.20 (brs, 2H, b $\left.{ }^{\prime}-\mathrm{H}\right), 7.45$ (brs, $\left.2 \mathrm{H}, \mathrm{b}_{1}{ }^{\prime}-\mathrm{H}\right), 7.46$ (t, $\left.{ }^{3} J=8.0 \mathrm{~Hz}, 2 \mathrm{H}, \mathrm{d}^{\prime}-\mathrm{H}\right), 7.52$ (brs, $\left.2 \mathrm{H}, \mathrm{c}^{\prime} / \mathrm{e}^{\prime}-\mathrm{H}\right)$, 7.65 (brs, 2H, e' $\left./ \mathrm{c}^{\prime}-\mathrm{H}\right), 7.99$ (d, $\left.{ }^{3} J=8.6 \mathrm{~Hz}, 2 \mathrm{H}, 8-\mathrm{H}\right), 8.14\left(\mathrm{~d},{ }^{3} J=8.8 \mathrm{~Hz}, 2 \mathrm{H}, 5 / 6-\mathrm{H}\right), 8.18\left(\mathrm{~d},{ }^{3} J=\right.$ $8.8 \mathrm{~Hz}, 2 \mathrm{H}, 6 / 5-\mathrm{H}), 8.63\left(\mathrm{~d},{ }^{3} J=8.6 \mathrm{~Hz}, 2 \mathrm{H}, 7-\mathrm{H}\right), 8.88$ (s, 2H, 4-H) ppm. ESI-MS: $m / z(\%) 836.2$ (100) $\left[\mathrm{Cu}_{2}(\mathbf{R 1})_{2}\right]^{2+}$. Elemental analysis: Calculated for $\mathrm{C}_{94} \mathrm{H}_{76} \mathrm{Br}_{2} \mathrm{Cu}_{2} \mathrm{~F}_{12} \mathrm{~N}_{6} \mathrm{O}_{6} \mathrm{P}_{2} \cdot \mathrm{CH}_{2} \mathrm{Cl}_{2} \cdot \mathrm{H}_{2} \mathrm{O}: \mathrm{C}$, 55.24; H, 3.90; N, 4.07. Found: C, 55.23; H, 3.59; N, 3.96. 


\section{Model Study}

In an NMR tube, 4-bromo-2-methylpyridine (1), 4-iodopyridine (2), phenanthroline 3, zinc porphyrin 4 , and $\left[\mathrm{Cu}\left(\mathrm{CH}_{3} \mathrm{CN}\right)_{4}\right] \mathrm{PF}_{6}(1.13 \mu \mathrm{mol})$ were mixed in a ratio of $1: 1: 1: 1: 1$. The mixture was dissolved in $\mathrm{CD}_{2} \mathrm{Cl}_{2}$. The subsequently measured ${ }^{1} \mathrm{H}$ NMR spectrum was compared with those of the individual complexes. Accordingly, the copper HETPYP complex $[\mathrm{Cu}(\mathbf{1})(\mathbf{2})]^{+}$and $(\mathbf{3} \cdot \mathbf{4})$ were afforded selectively, as indicated by NMR.
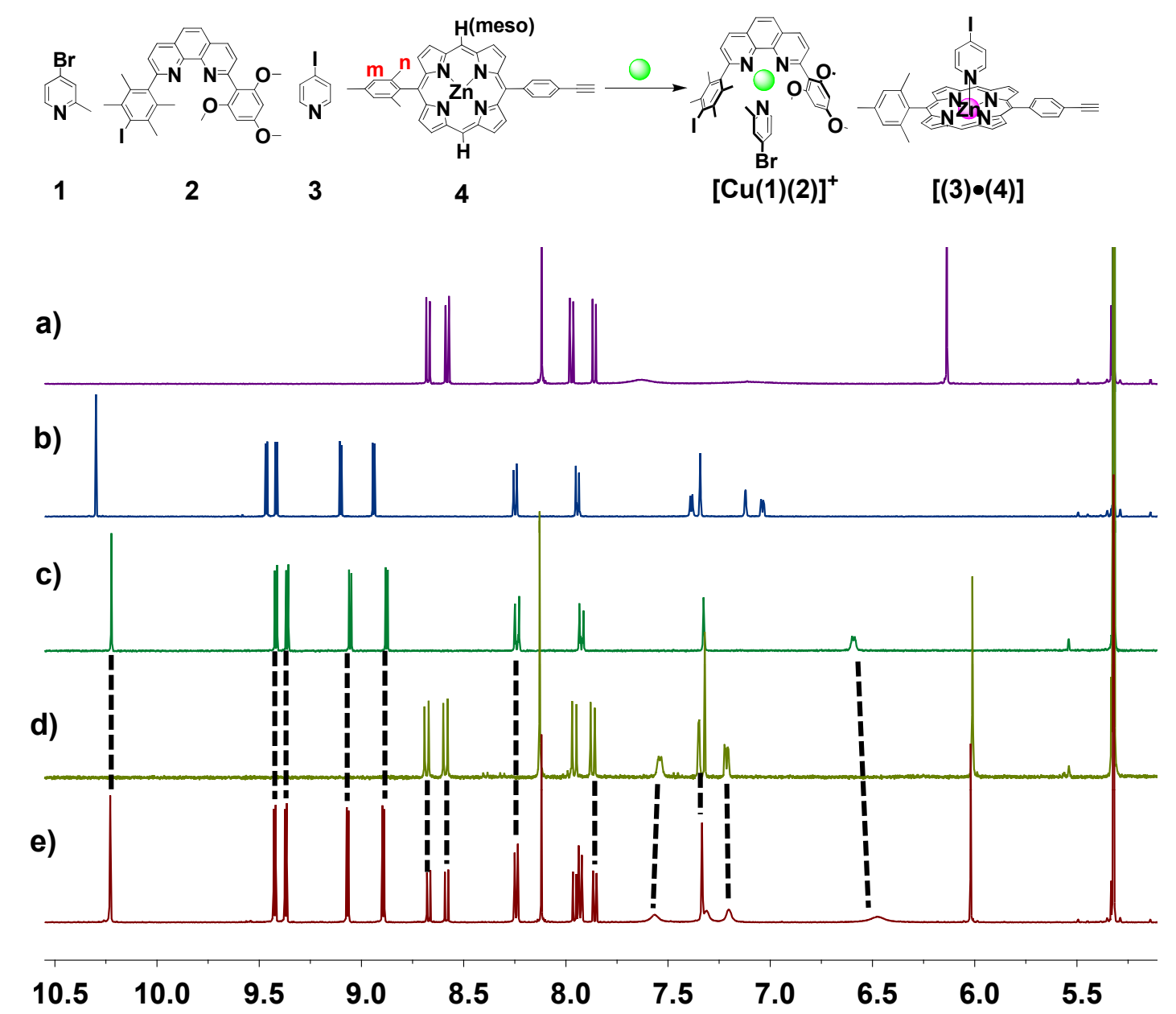

Figure S1. ${ }^{1} \mathrm{H}$ NMR $(400 \mathrm{MHz}, 298 \mathrm{~K})$ of (a) $[\mathrm{Cu}(\mathbf{2})(\mathbf{3})]^{+}$; (b) $[(\mathbf{1}) \cdot(\mathbf{4})]$; (c) [(3) $\left.\bullet(\mathbf{4})\right]$; (d) $[\mathrm{Cu}(\mathbf{1})(\mathbf{2})]^{+}$; (e) after mixing of $\mathbf{1}, \mathbf{2}, \mathbf{3}, \mathbf{4}$ and $\left[\mathrm{Cu}\left(\mathrm{CH}_{3} \mathrm{CN}\right)_{4}\right] \mathrm{PF}_{6}$ in a ratio of 1:1:1:1:1 in $\mathrm{CD}_{2} \mathrm{Cl}_{2}$ furnishing a mixture of $[\mathrm{Cu}(\mathbf{1})(\mathbf{2})]^{+}$and $[(\mathbf{3}) \cdot(\mathbf{4})]$. 


\section{NMR Spectra}
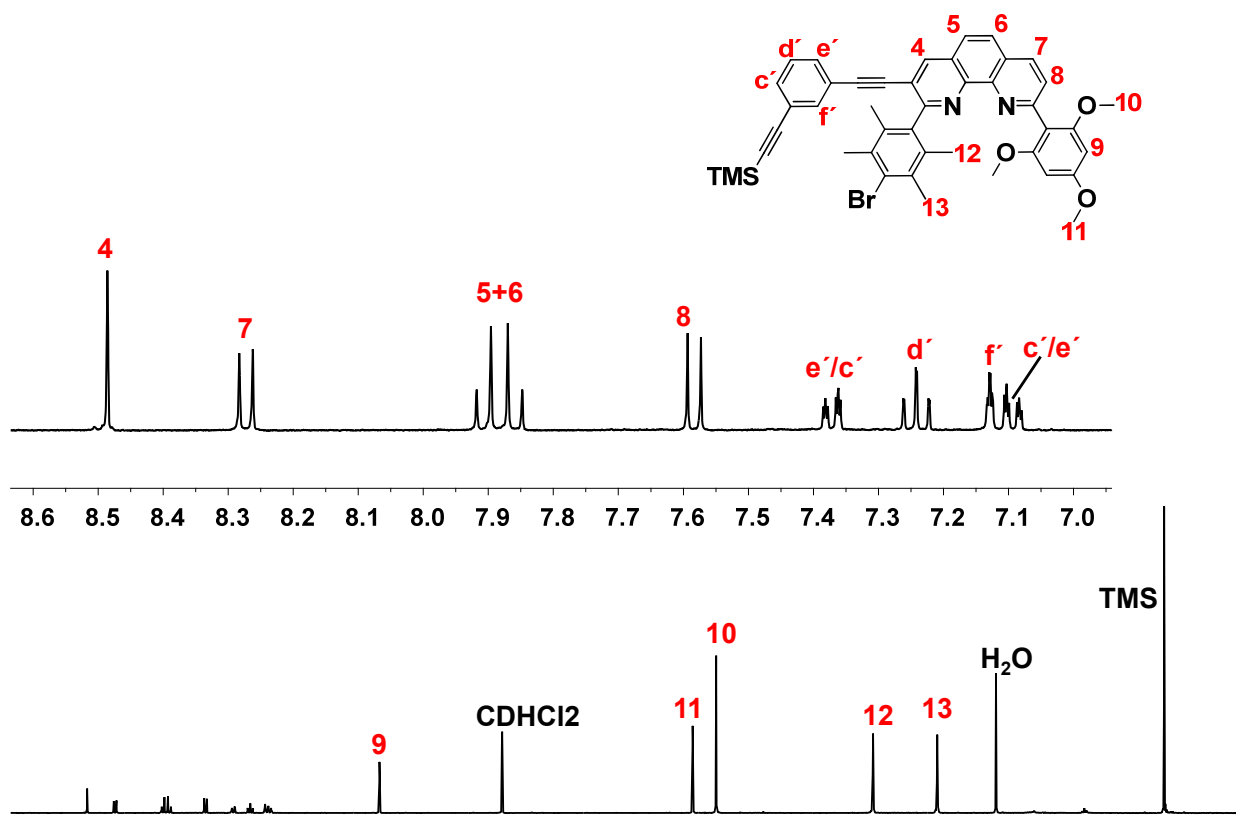

$\begin{array}{lllllllllllllllllll}9.0 & 8.5 & 8.0 & 7.5 & 7.0 & 6.5 & 6.0 & 5.5 & 5.0 & 4.5 & 4.0 & 3.5 & 3.0 & 2.5 & 2.0 & 1.5 & 1.0 & 0.5 & 0.0\end{array}$

Figure S2. ${ }^{1} \mathrm{H}$ NMR spectrum of $\mathbf{1 0}$ in $\mathrm{CD}_{2} \mathrm{Cl}_{2}(400 \mathrm{MHz}, 298 \mathrm{~K})$.

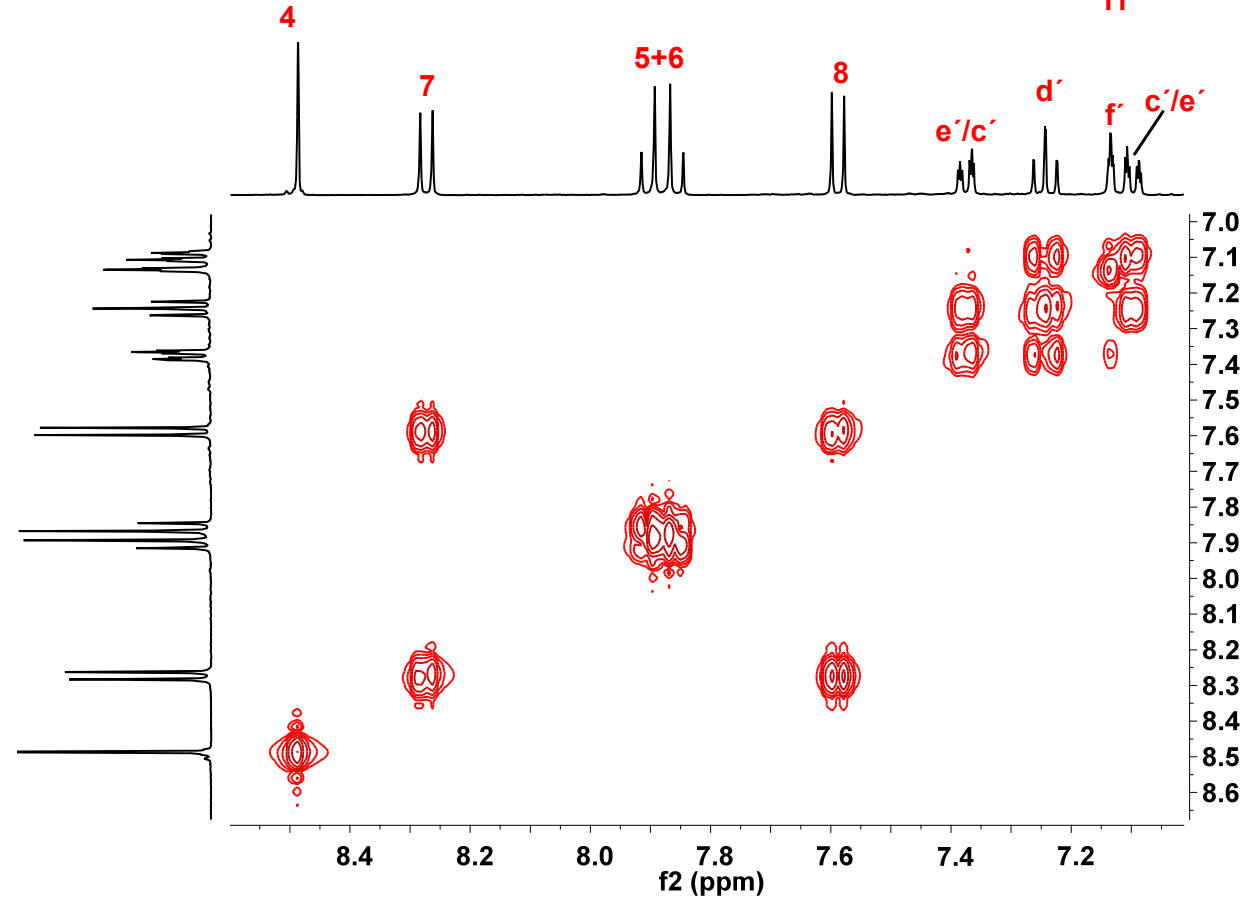

Figure S3. ${ }^{1} \mathrm{H}-{ }^{1} \mathrm{H}$ COSY spectrum of 10 in $\mathrm{CD}_{2} \mathrm{Cl}_{2}(400 \mathrm{MHz}, 298 \mathrm{~K})$. 

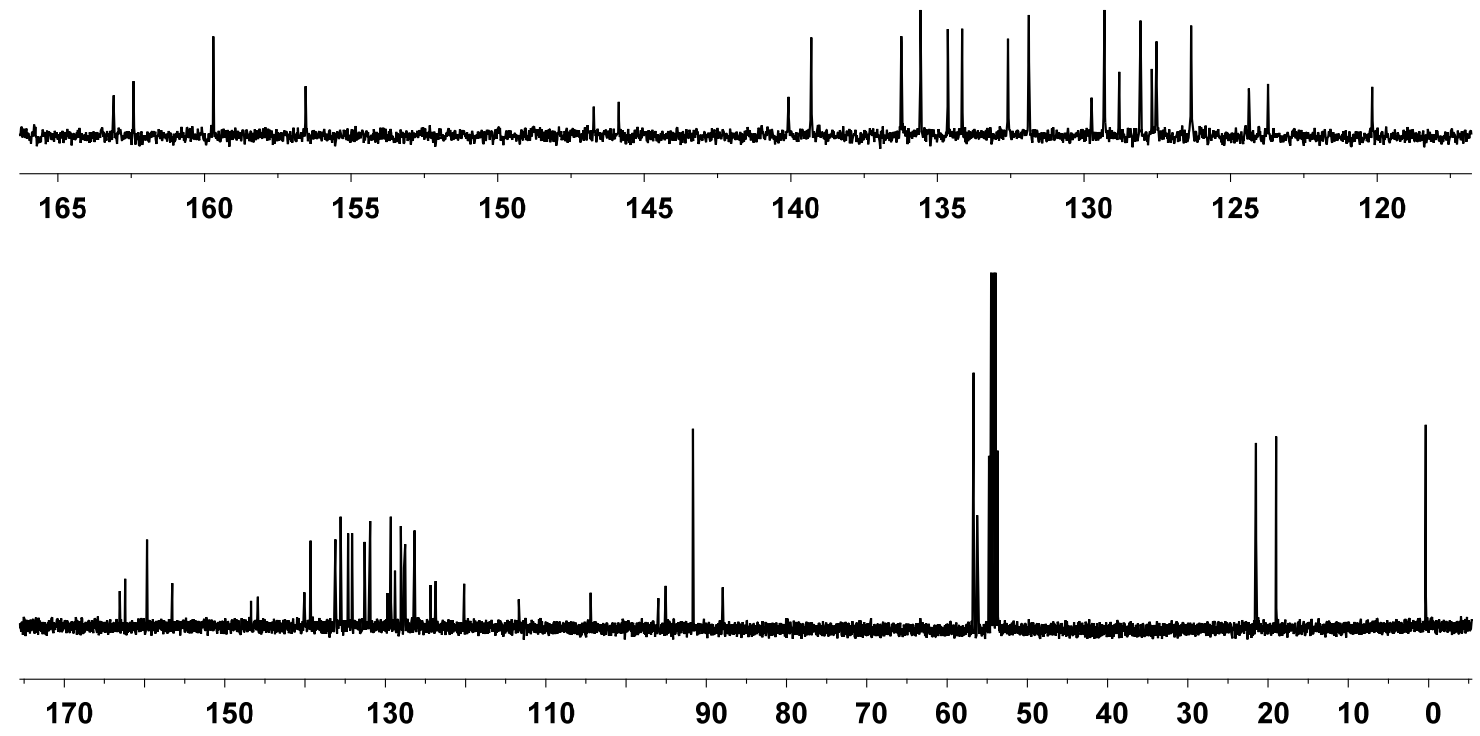

Figure S4. ${ }^{13} \mathrm{C}$ NMR spectrum of $\mathbf{1 0}$ in $\mathrm{CD}_{2} \mathrm{Cl}_{2}(100 \mathrm{MHz}, 298 \mathrm{~K})$.
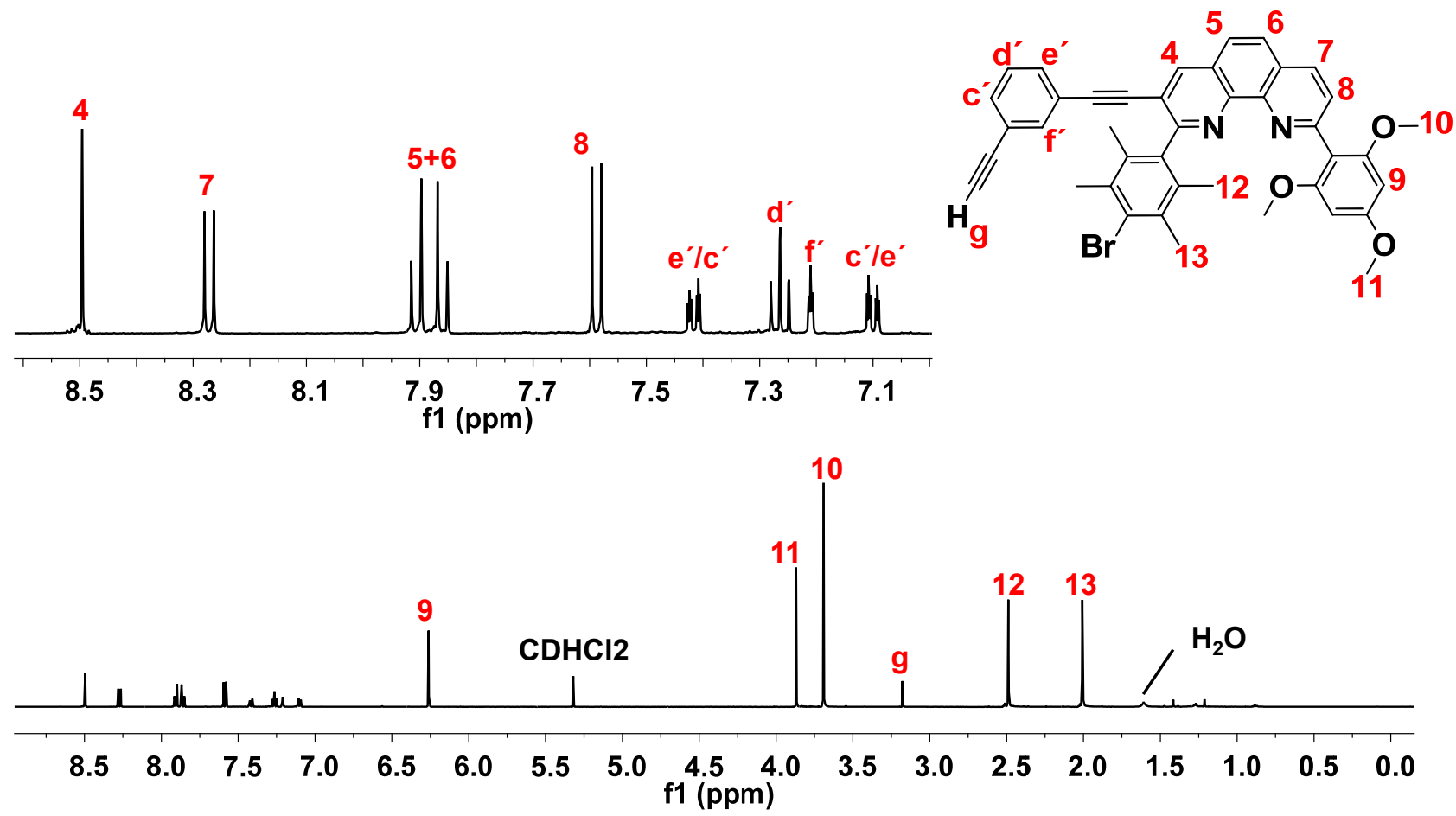

Figure S5. ${ }^{1} \mathrm{H}$ NMR spectrum of $\mathbf{1 1}$ in $\mathrm{CD}_{2} \mathrm{Cl}_{2}(400 \mathrm{MHz}, 298 \mathrm{~K})$. 


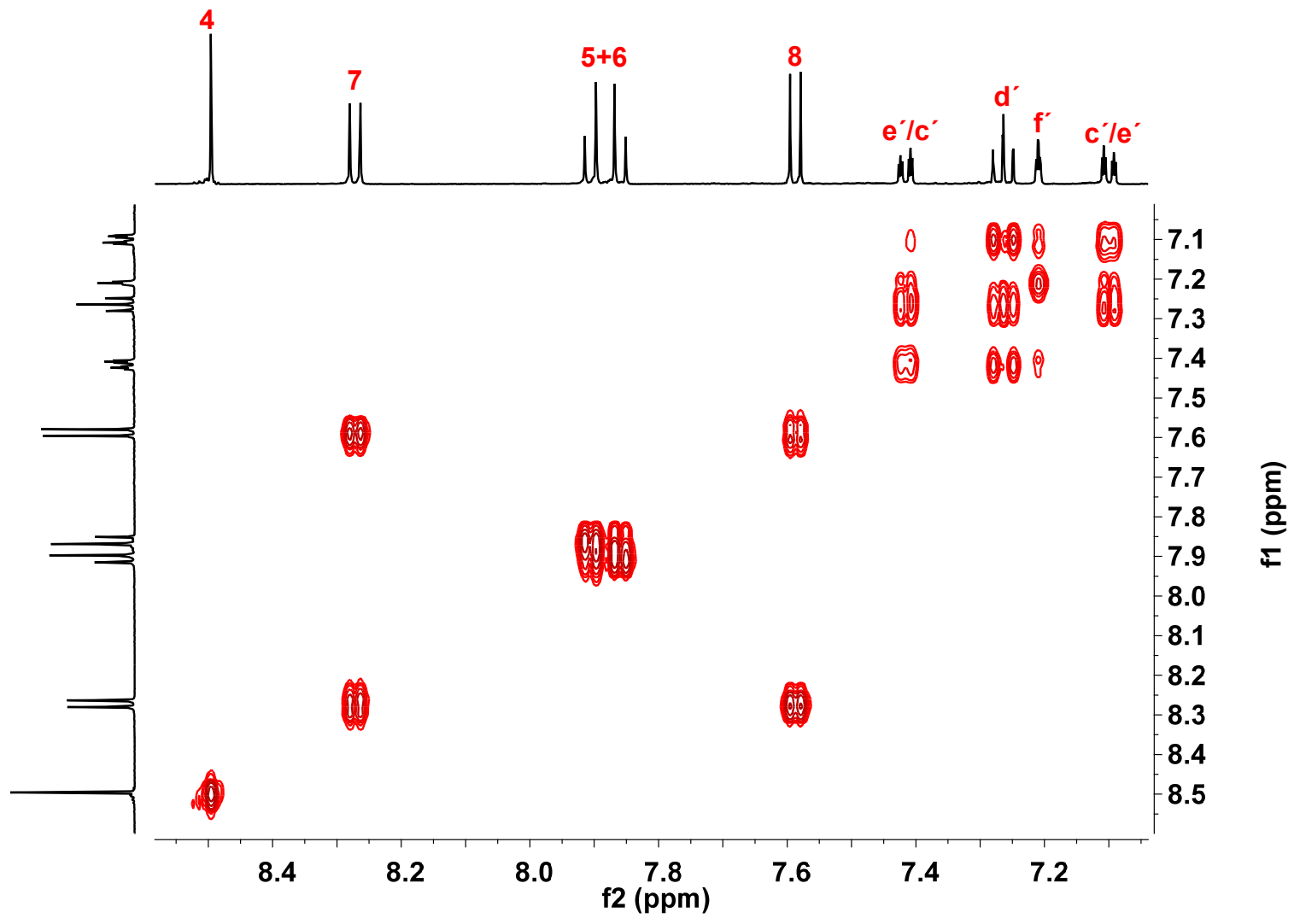

Figure S6. ${ }^{1} \mathrm{H}-{ }^{1} \mathrm{H}$ COSY spectrum of 11 in $\mathrm{CD}_{2} \mathrm{Cl}_{2}(400 \mathrm{MHz}, 298 \mathrm{~K})$.

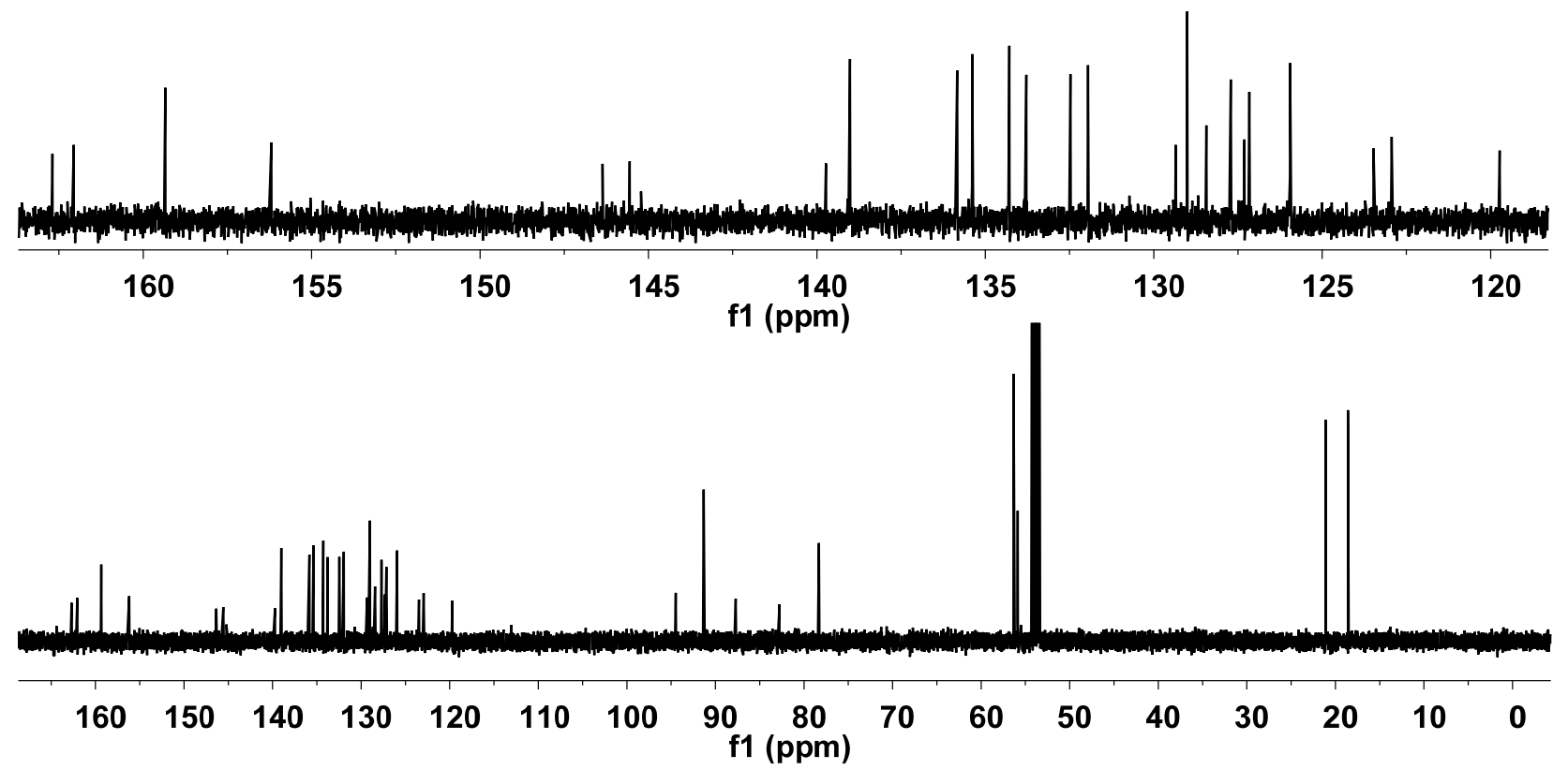

Figure S7. ${ }^{13} \mathrm{C}$ NMR spectrum of $\mathbf{1 1}$ in $\mathrm{CD}_{2} \mathrm{Cl}_{2}(100 \mathrm{MHz}, 298 \mathrm{~K})$. 


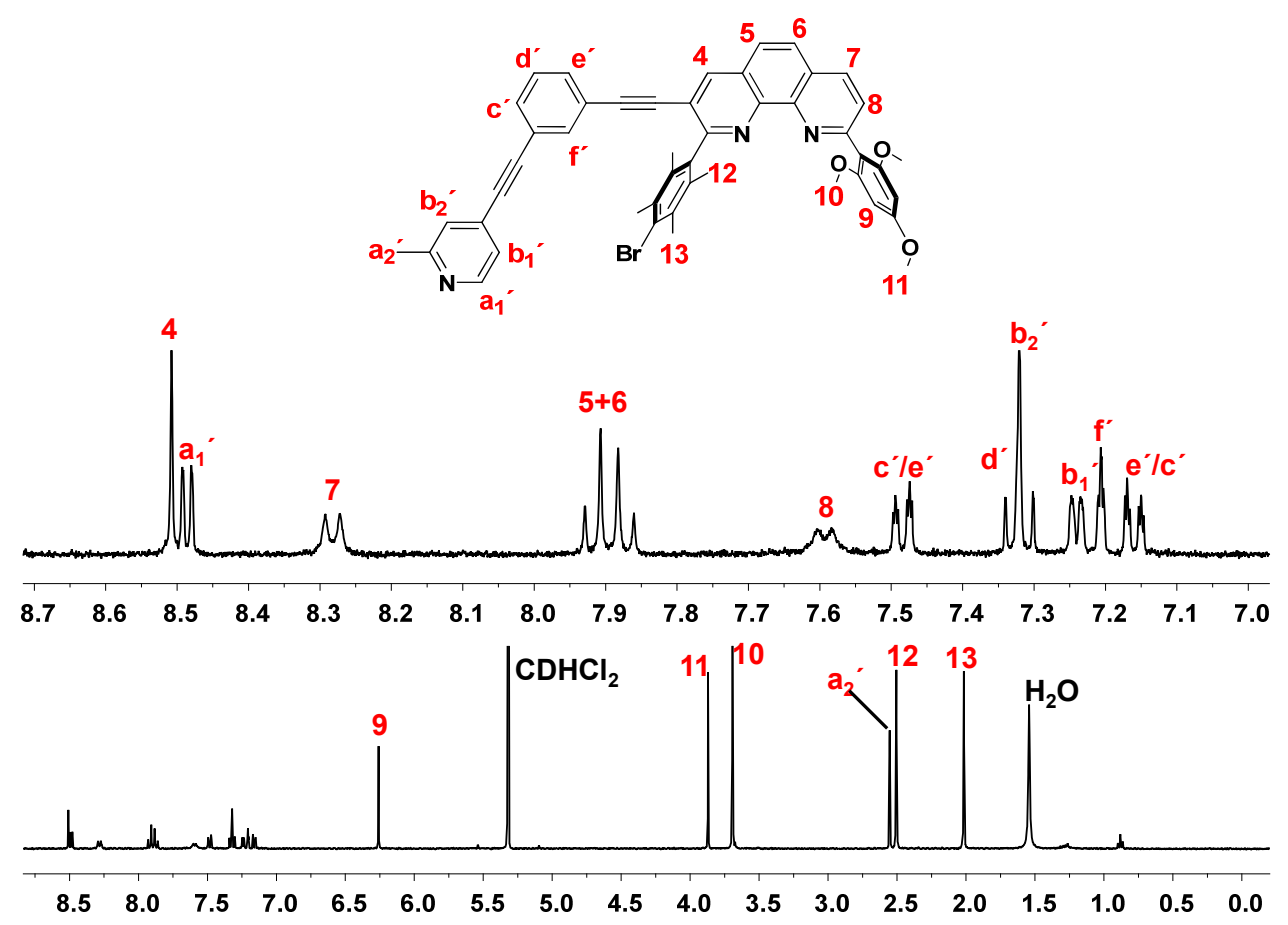

Figure S8. ${ }^{1} \mathrm{H}$ NMR spectrum of $\mathbf{R} 1$ in $\mathrm{CD}_{2} \mathrm{Cl}_{2}(400 \mathrm{MHz}, 298 \mathrm{~K})$.

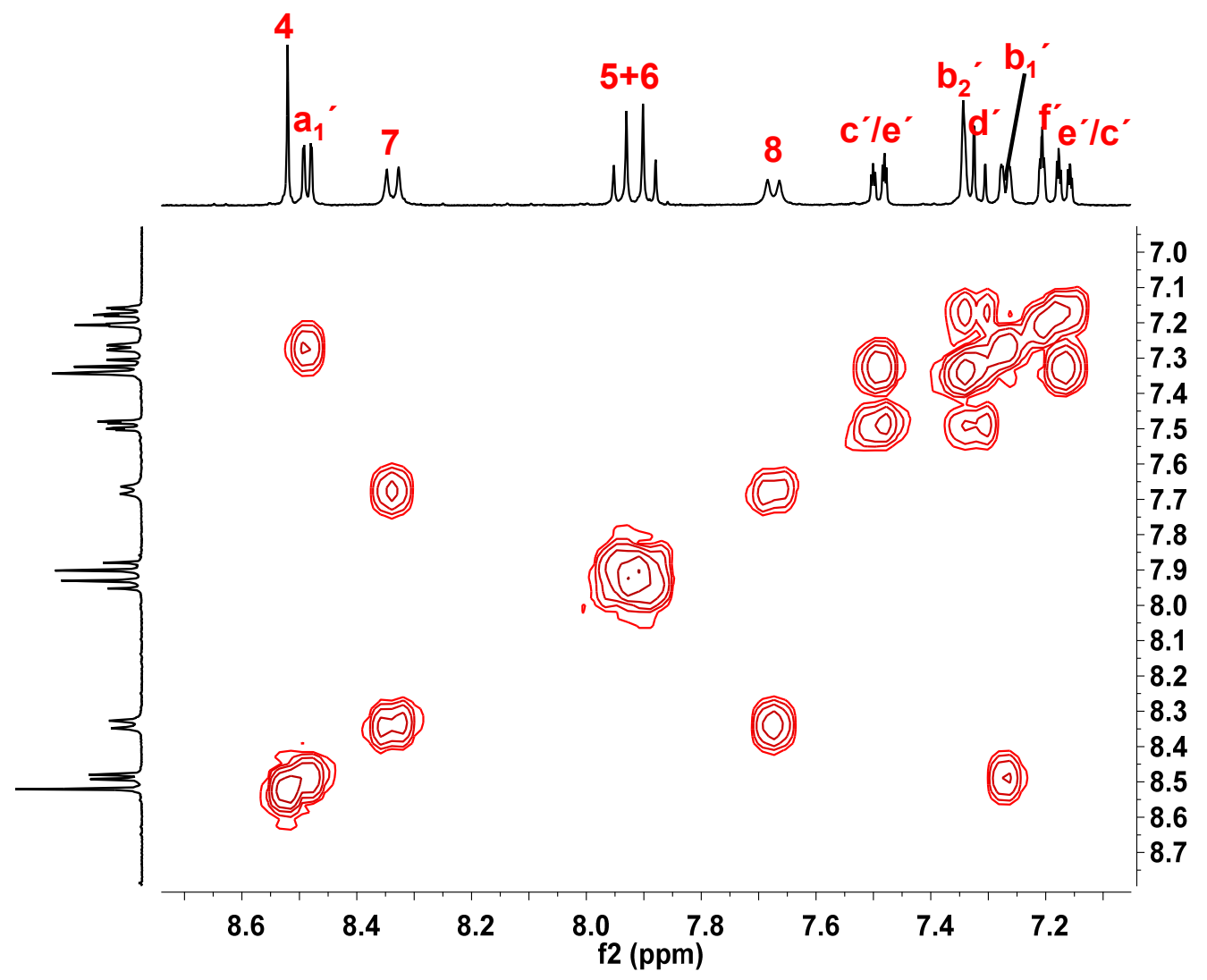

Figure S9. ${ }^{1} \mathrm{H}-{ }^{1} \mathrm{H}$ COSY spectrum of $\mathbf{R} 1$ in $\mathrm{CD}_{2} \mathrm{Cl}_{2}(400 \mathrm{MHz}, 298 \mathrm{~K})$. 

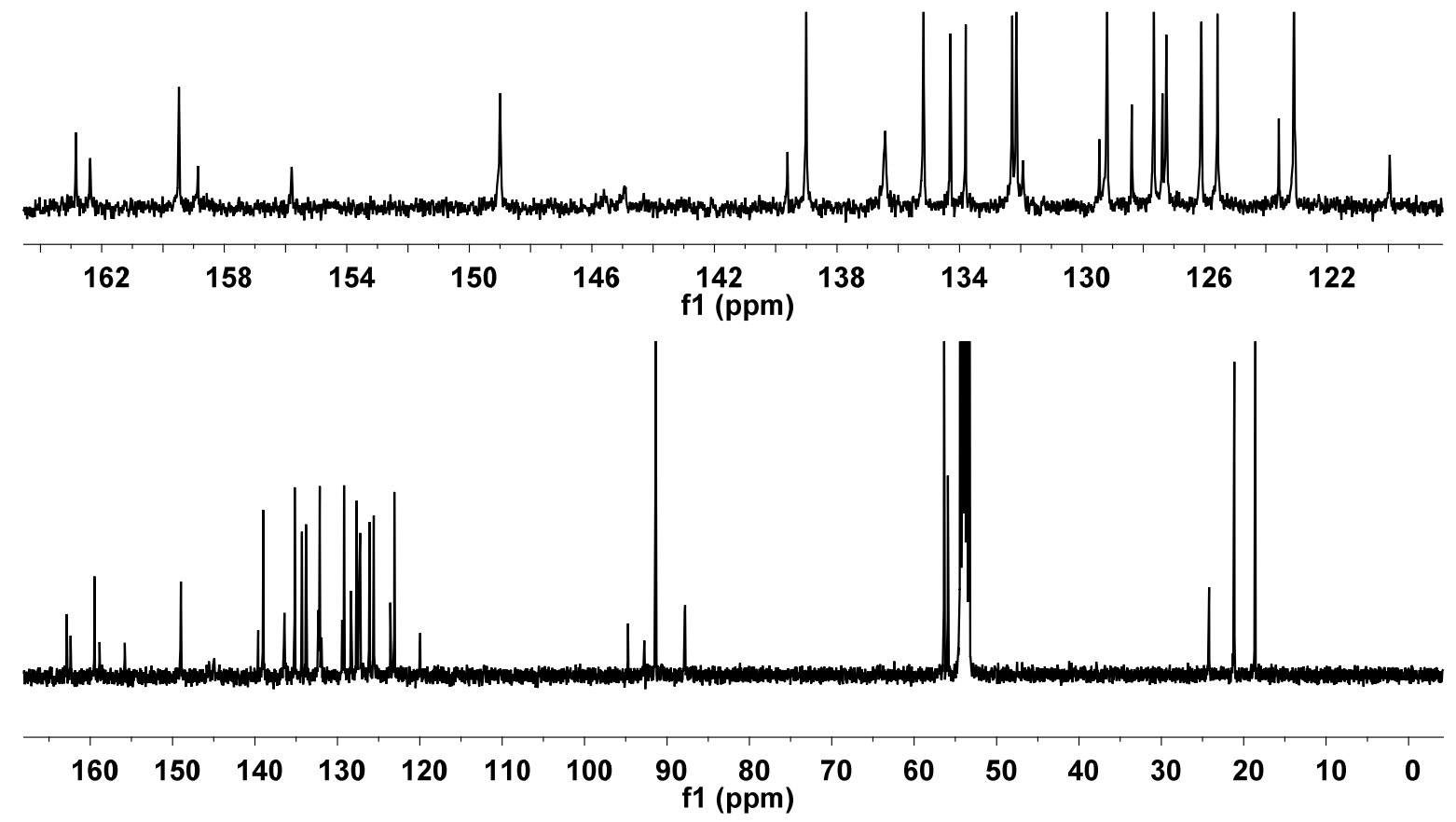

Figure S10. ${ }^{13} \mathrm{C}$ NMR spectrum of $\mathbf{R} 1$ in $\mathrm{CD}_{2} \mathrm{Cl}_{2}(100 \mathrm{MHz}, 298 \mathrm{~K})$.
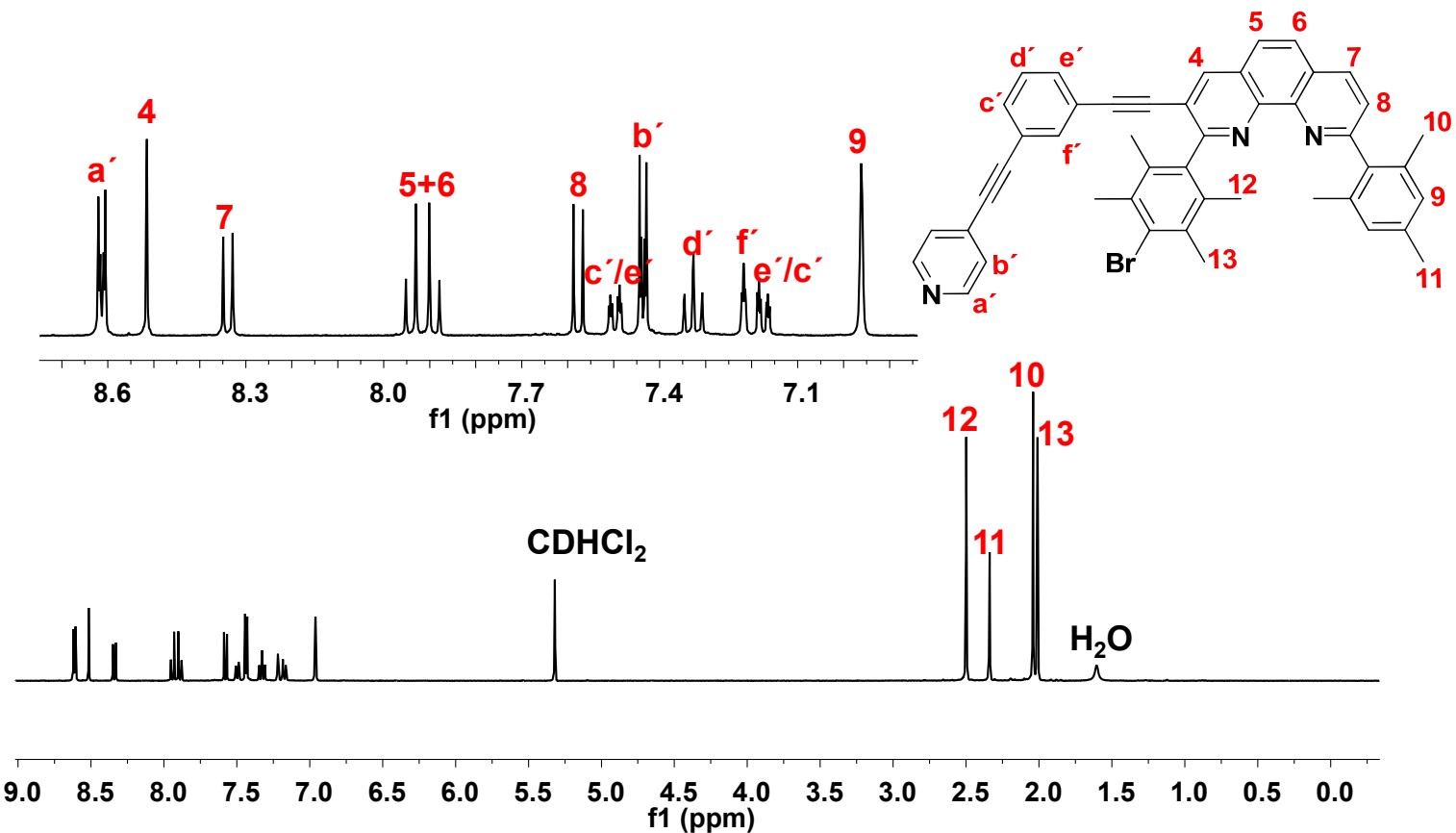

Figure S11. ${ }^{1} \mathrm{H}$ NMR spectrum of $\mathbf{R} 2$ in $\mathrm{CD}_{2} \mathrm{Cl}_{2}(400 \mathrm{MHz}, 298 \mathrm{~K})$. 


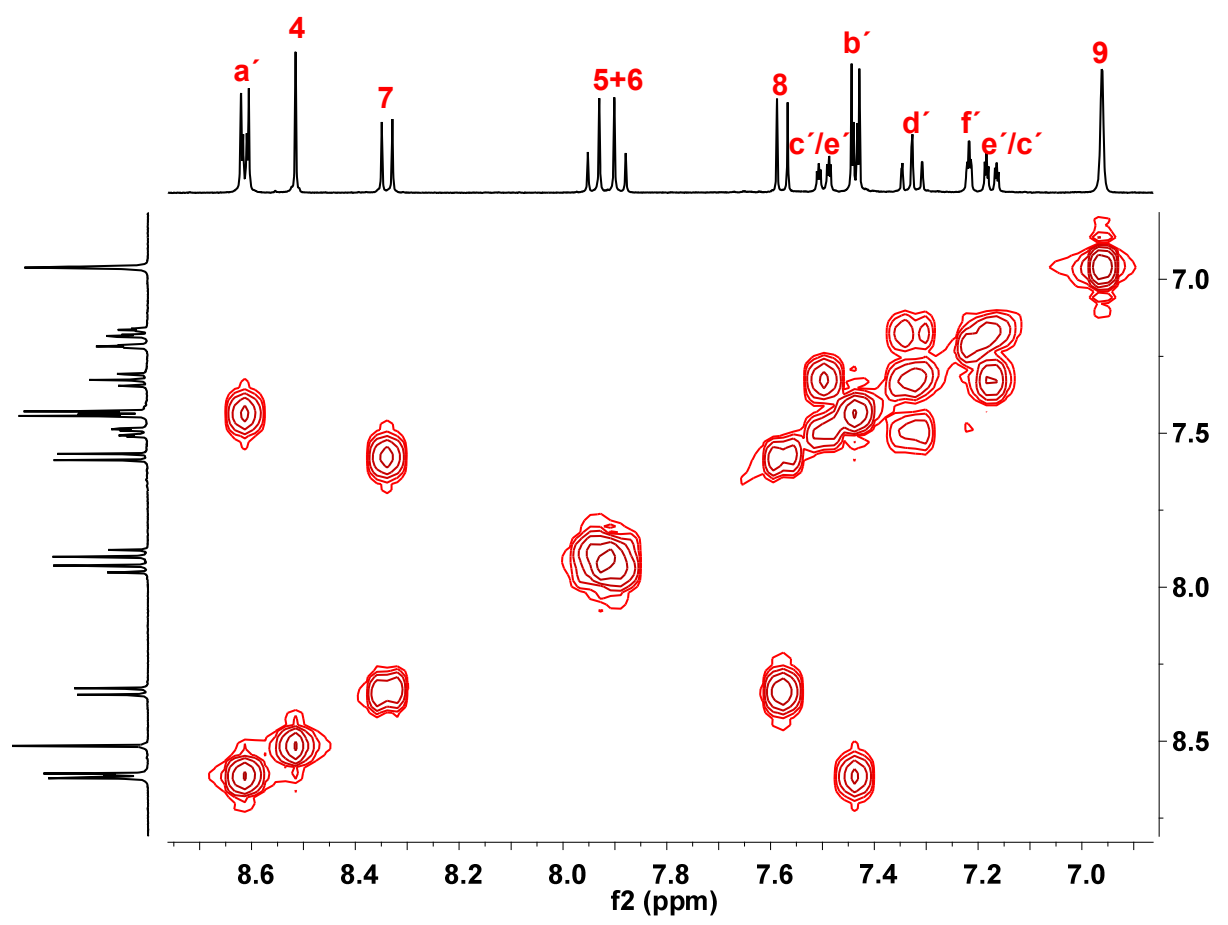

Figure S12. ${ }^{1} \mathrm{H}-{ }^{1} \mathrm{H}$ COSY spectrum of $\mathbf{R} 2$ in $\mathrm{CD}_{2} \mathrm{Cl}_{2}(400 \mathrm{MHz}, 298 \mathrm{~K})$.

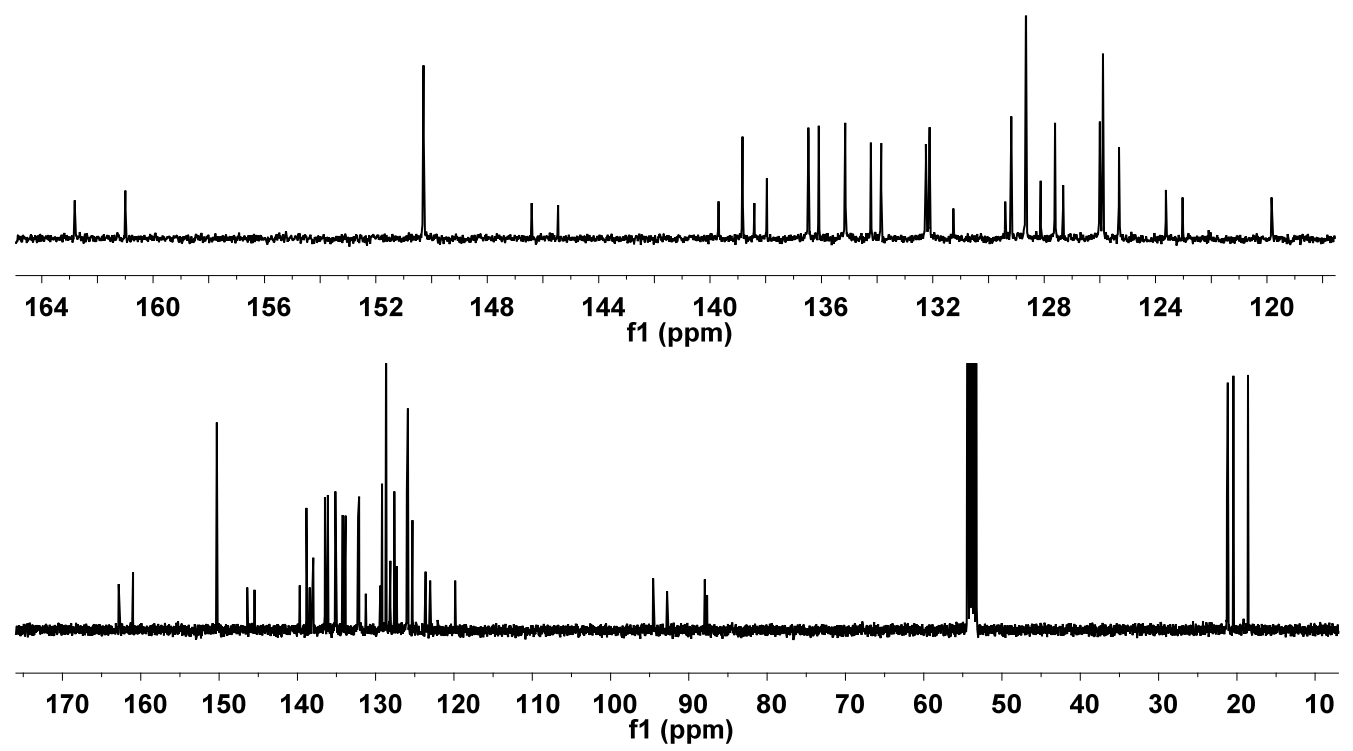

Figure S13. ${ }^{13} \mathrm{C}$ NMR spectrum of $\mathbf{R 2}$ in $\mathrm{CD}_{2} \mathrm{Cl}_{2}(100 \mathrm{MHz}, 298 \mathrm{~K})$. 

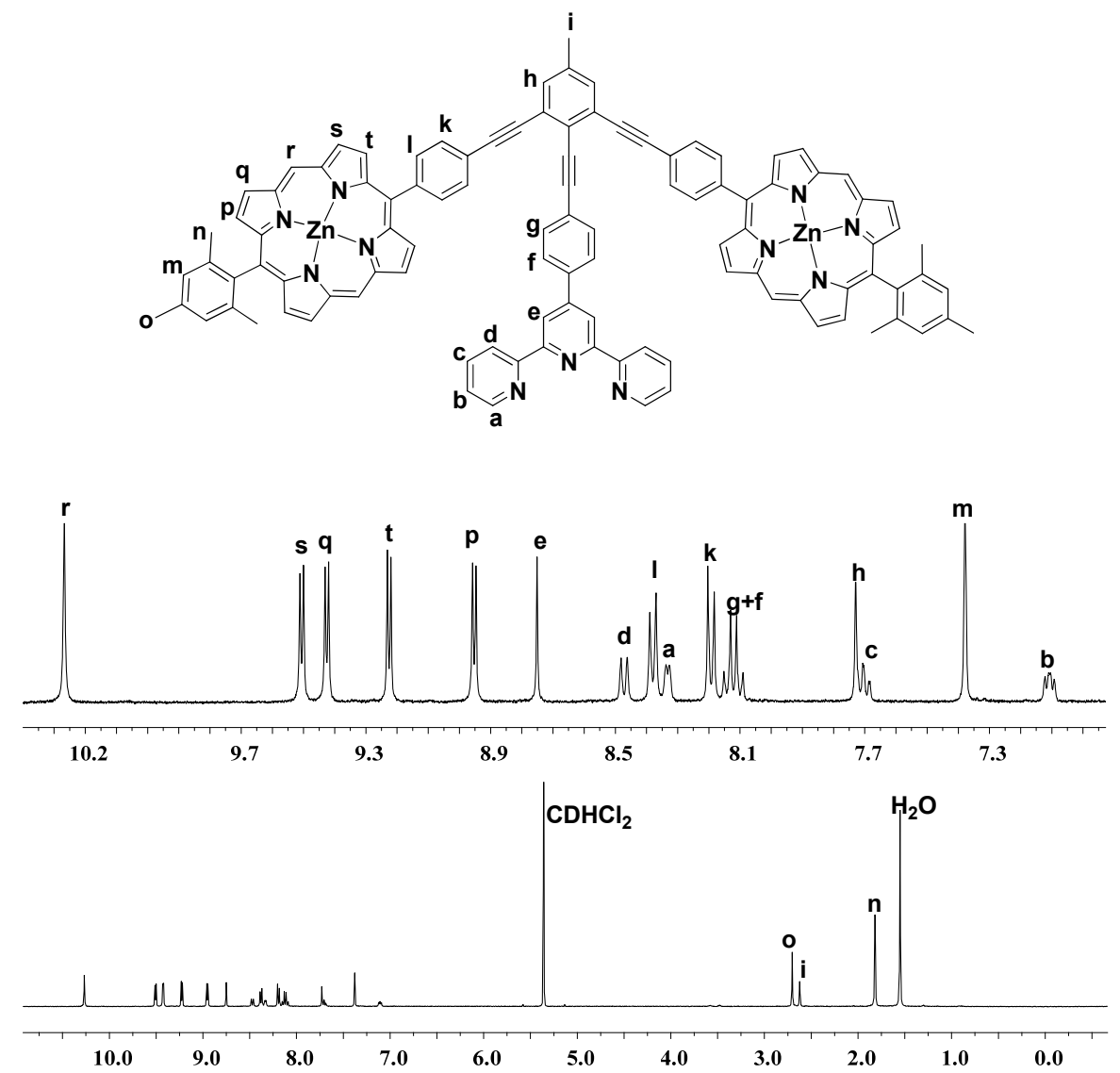

Figure S14. ${ }^{1} \mathrm{H}$ NMR spectrum of stator $\mathbf{S}$ in $\mathrm{CD}_{2} \mathrm{Cl}_{2}(400 \mathrm{MHz}, 298 \mathrm{~K})$.

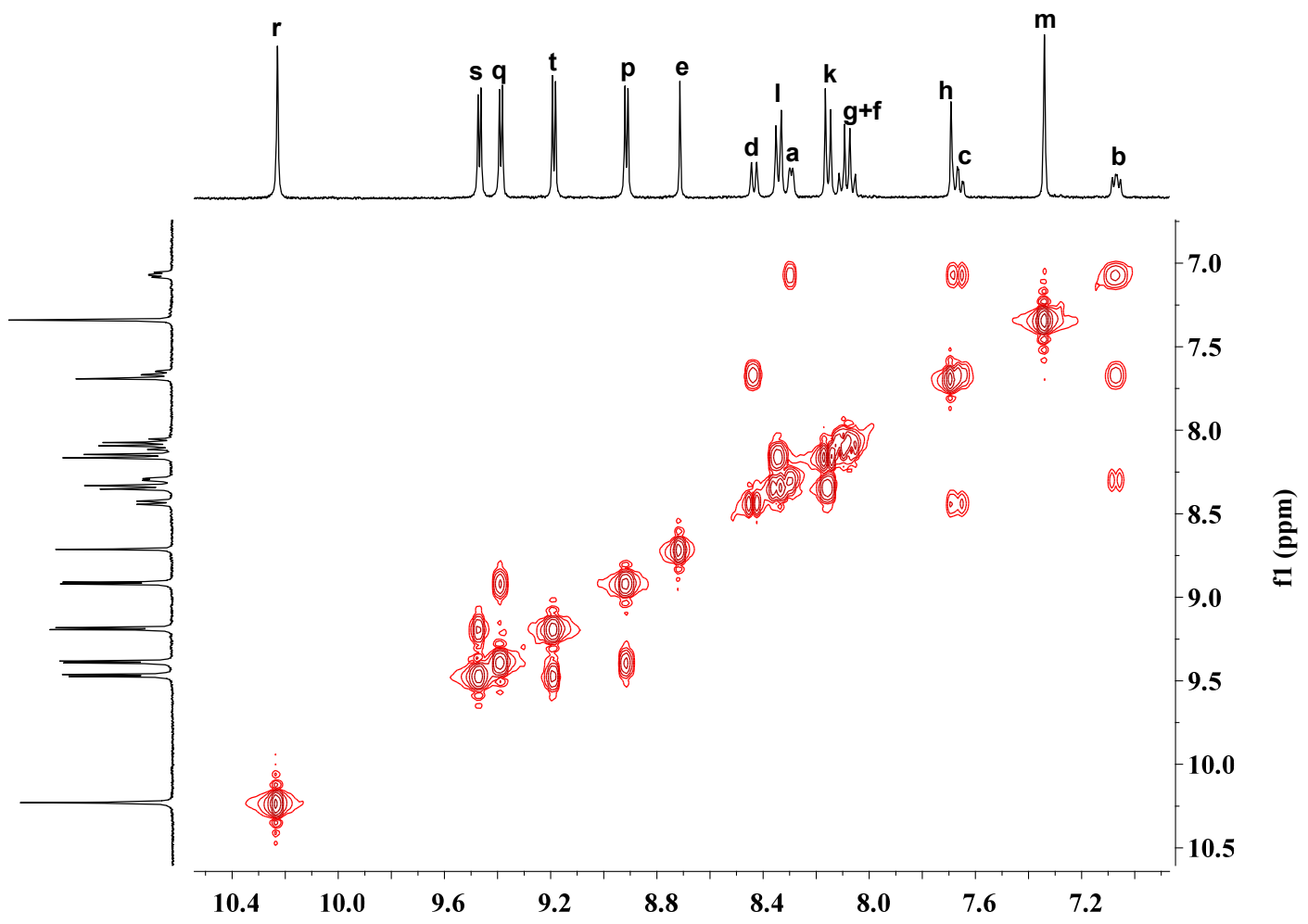

Figure S15. ${ }^{1} \mathrm{H}-{ }^{1} \mathrm{H}$ COSY spectrum of stator $\mathbf{S}$ in $\mathrm{CD}_{2} \mathrm{Cl}_{2}(400 \mathrm{MHz}, 298 \mathrm{~K})$. 

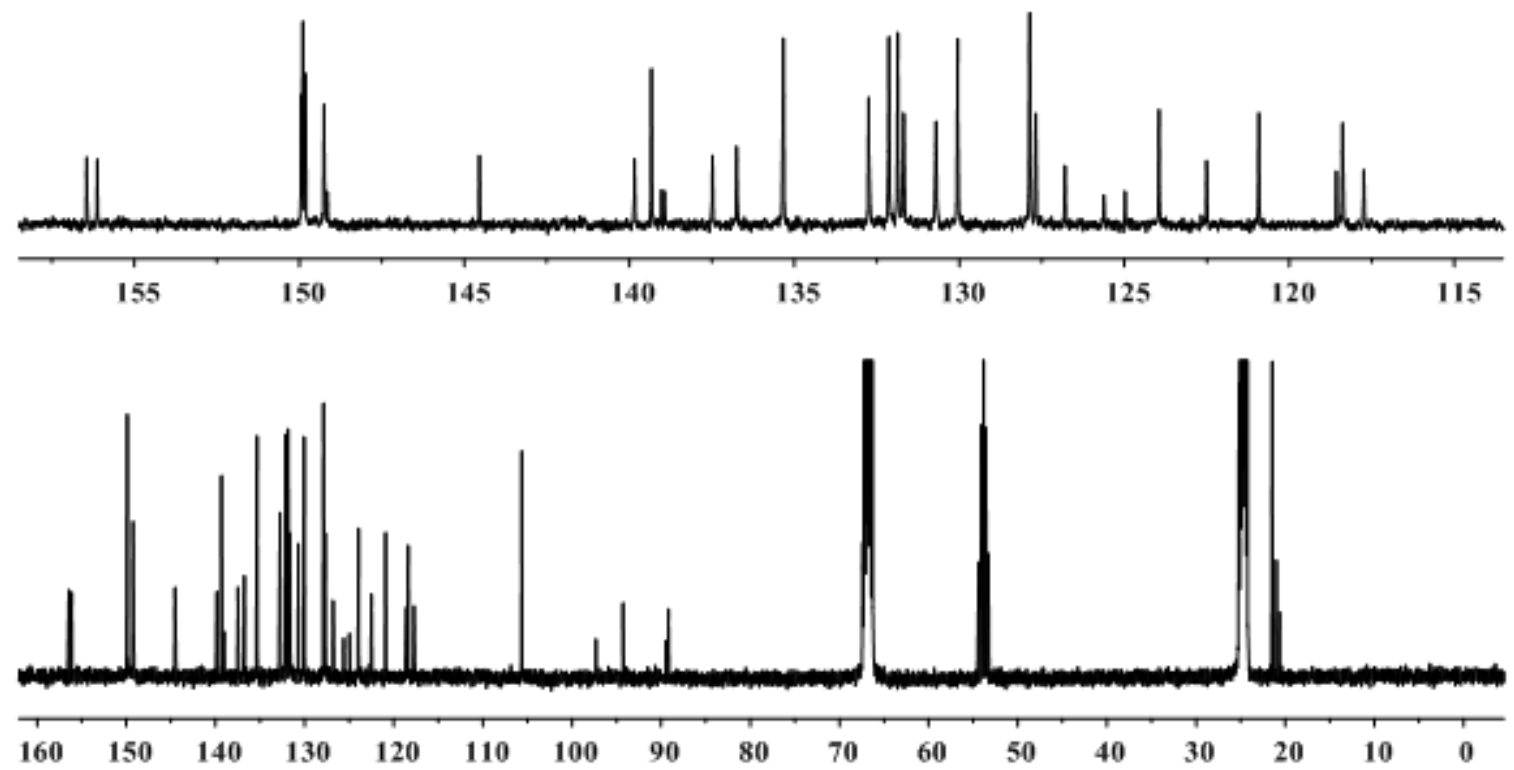

Figure S16. ${ }^{13} \mathrm{C}$ NMR spectrum of stator $\mathbf{S}$ in THF-d $\mathrm{d}_{8}: \mathrm{CD}_{2} \mathrm{Cl}_{2}(9: 1)(100 \mathrm{MHz}, 298 \mathrm{~K})$.
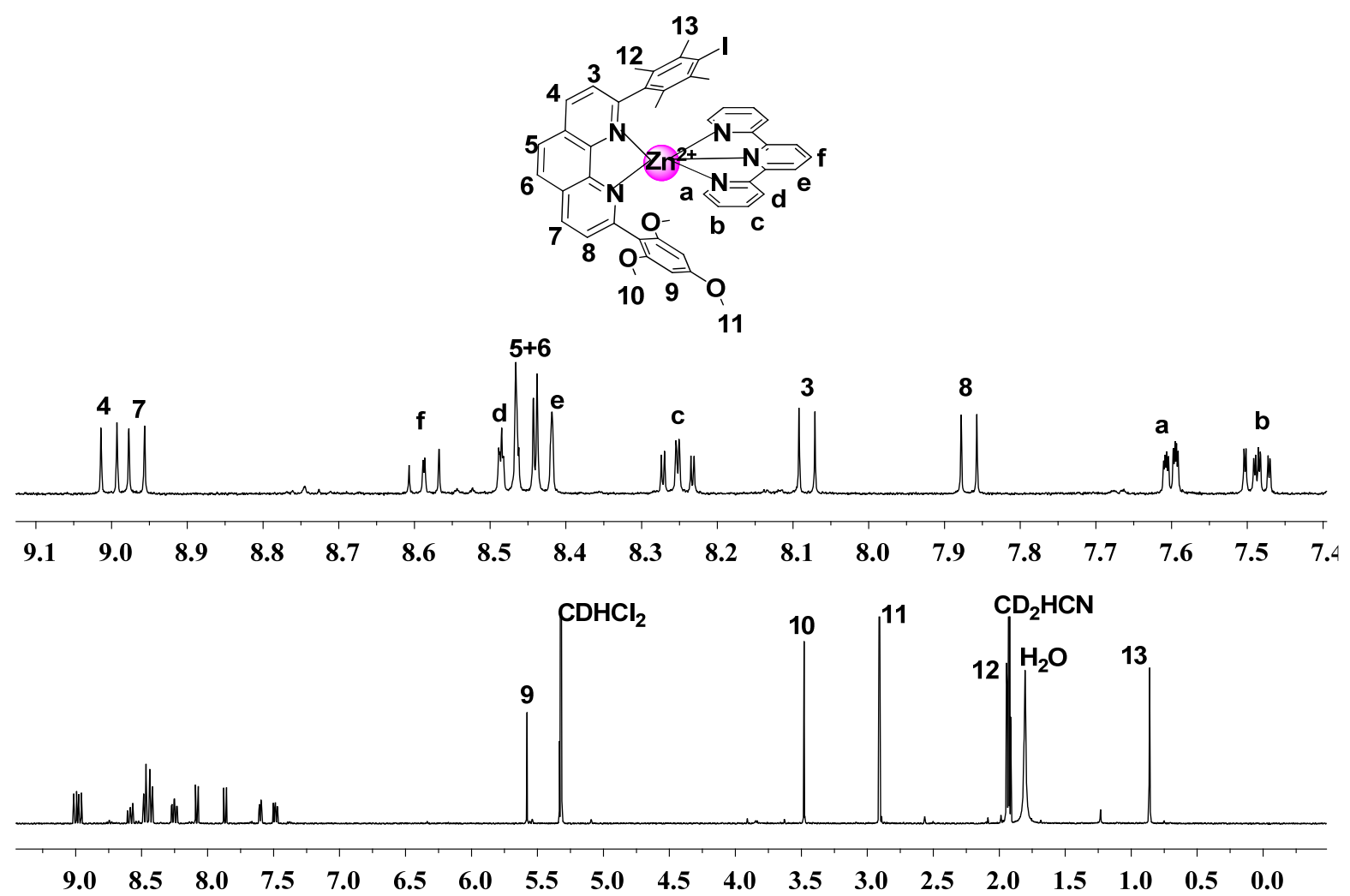

Figure S17. ${ }^{1} \mathrm{H}$ NMR spectrum of $[\mathrm{Zn}(\mathbf{2})(\mathbf{6})]^{2+}$ in $\mathrm{CD}_{2} \mathrm{Cl}_{2}: \mathrm{CD}_{3} \mathrm{CN}(10: 1)(400 \mathrm{MHz}, 298 \mathrm{~K})$. 


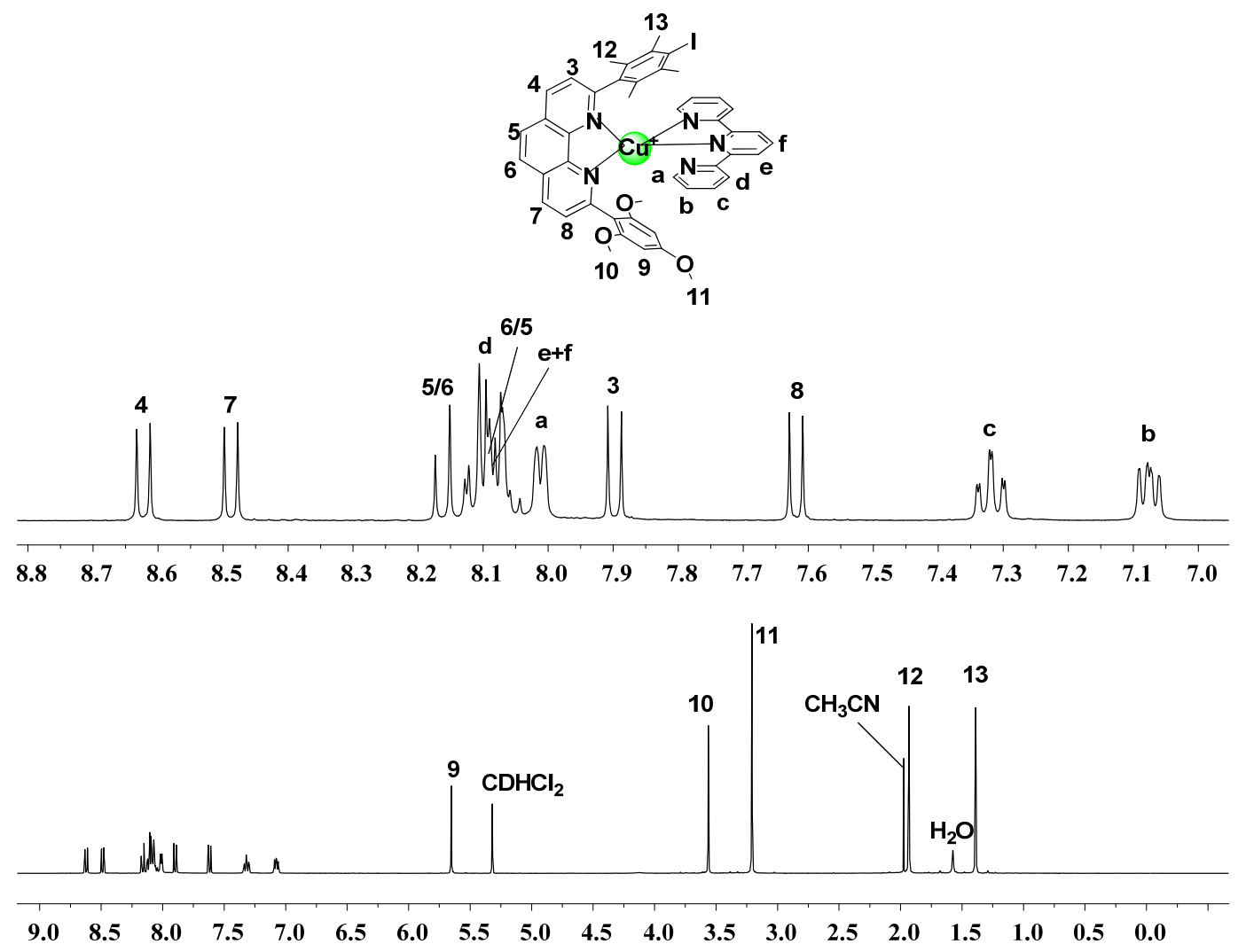

Figure S18. ${ }^{1} \mathrm{H}$ NMR spectrum of $[\mathrm{Cu}(\mathbf{2})(\mathbf{6})]^{+}$in $\mathrm{CD}_{2} \mathrm{Cl}_{2}(400 \mathrm{MHz}, 298 \mathrm{~K})$.

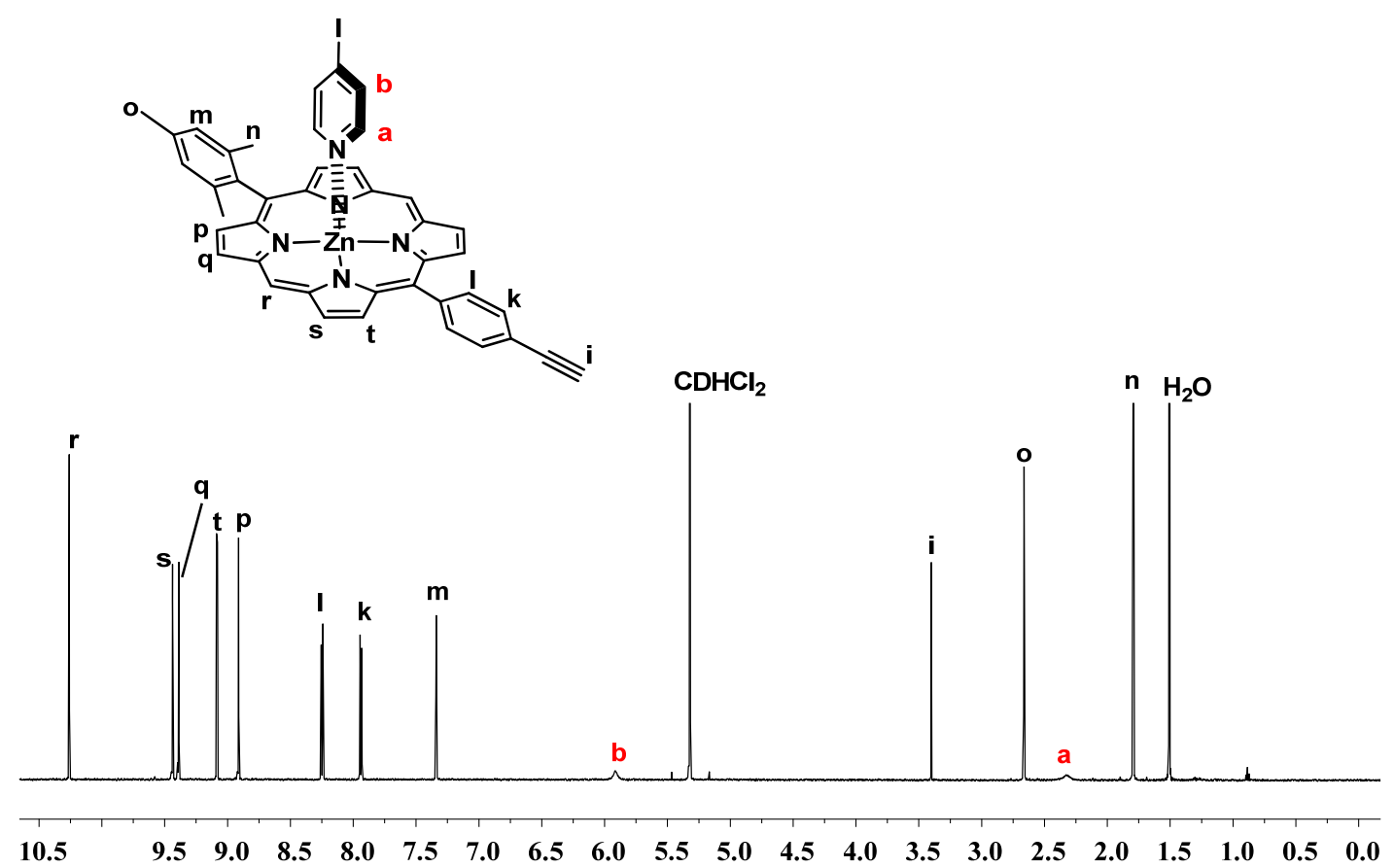

Figure S19. ${ }^{1} \mathrm{H}$ NMR spectrum of $[(\mathbf{3}) \bullet(4)]$ in $\mathrm{CD}_{2} \mathrm{Cl}_{2}(400 \mathrm{MHz}, 298 \mathrm{~K})$. 

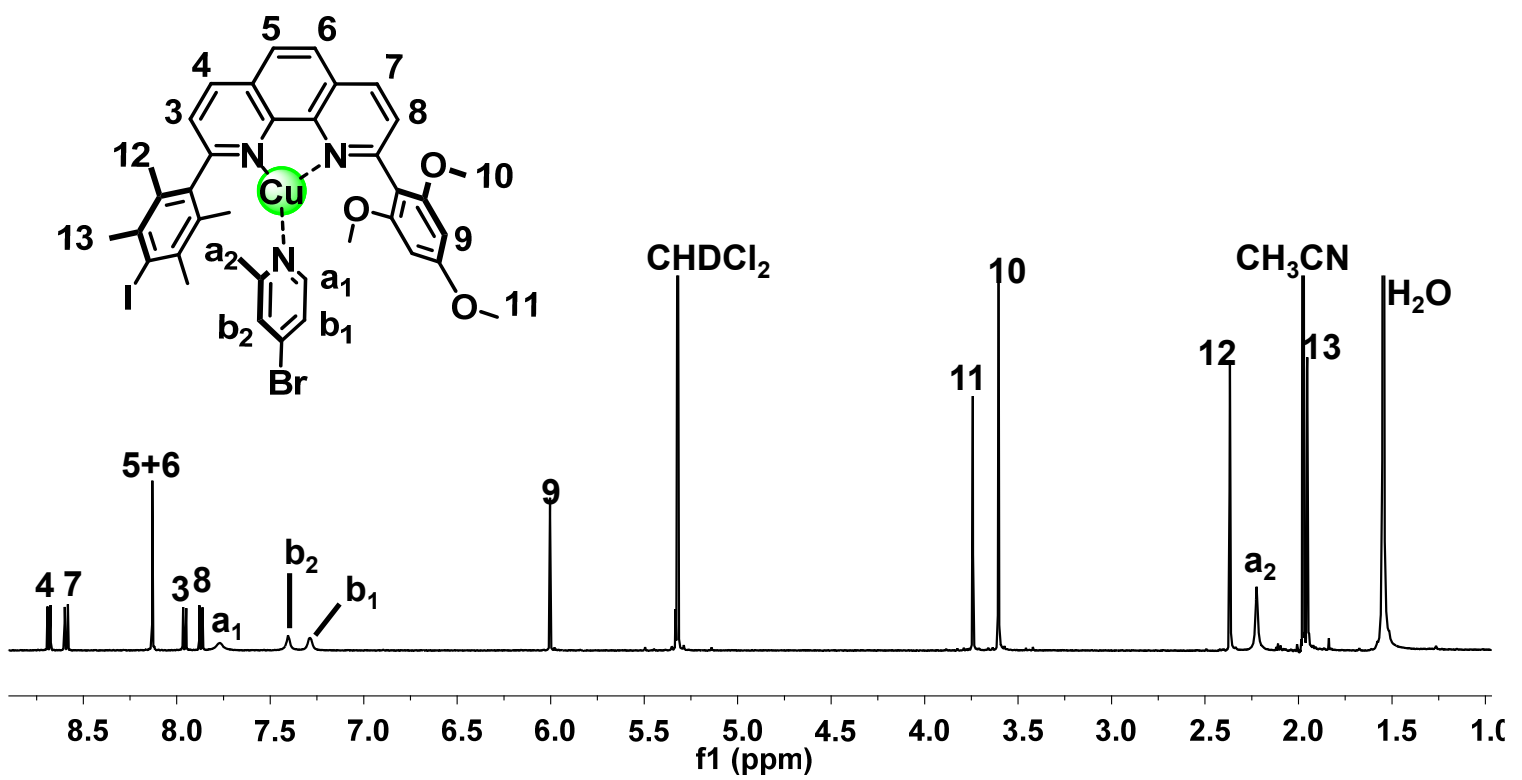

Figure S20. ${ }^{1} \mathrm{H}$ NMR spectrum of $[\mathrm{Cu}(\mathbf{1})(\mathbf{2})]^{+}$in $\mathrm{CD}_{2} \mathrm{Cl}_{2}(400 \mathrm{MHz}, 298 \mathrm{~K})$.

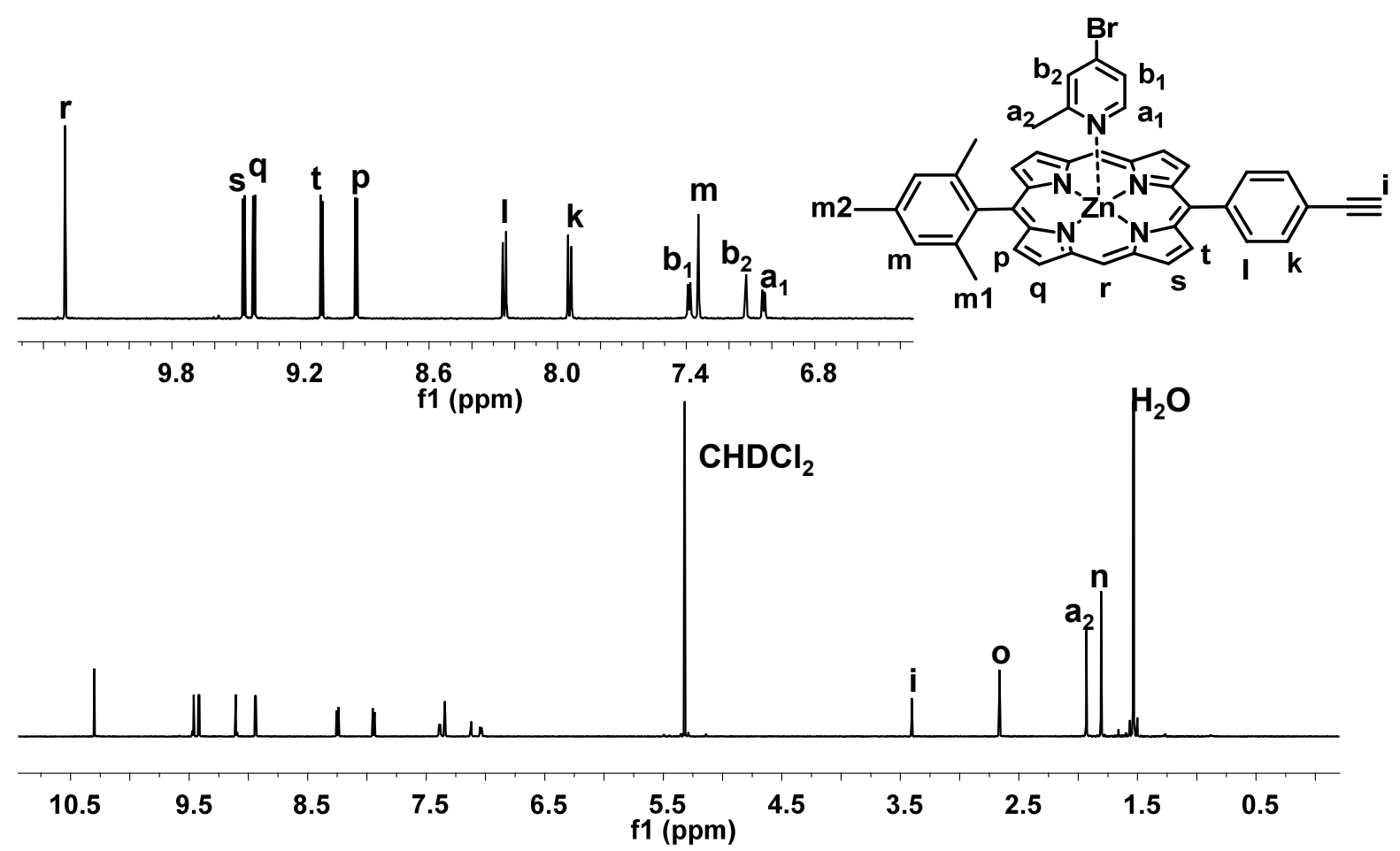

Figure S21. ${ }^{1} \mathrm{H}$ NMR spectrum of $[(\mathbf{1}) \bullet(4)]$ in $\mathrm{CD}_{2} \mathrm{Cl}_{2}(400 \mathrm{MHz}, 298 \mathrm{~K})$. 

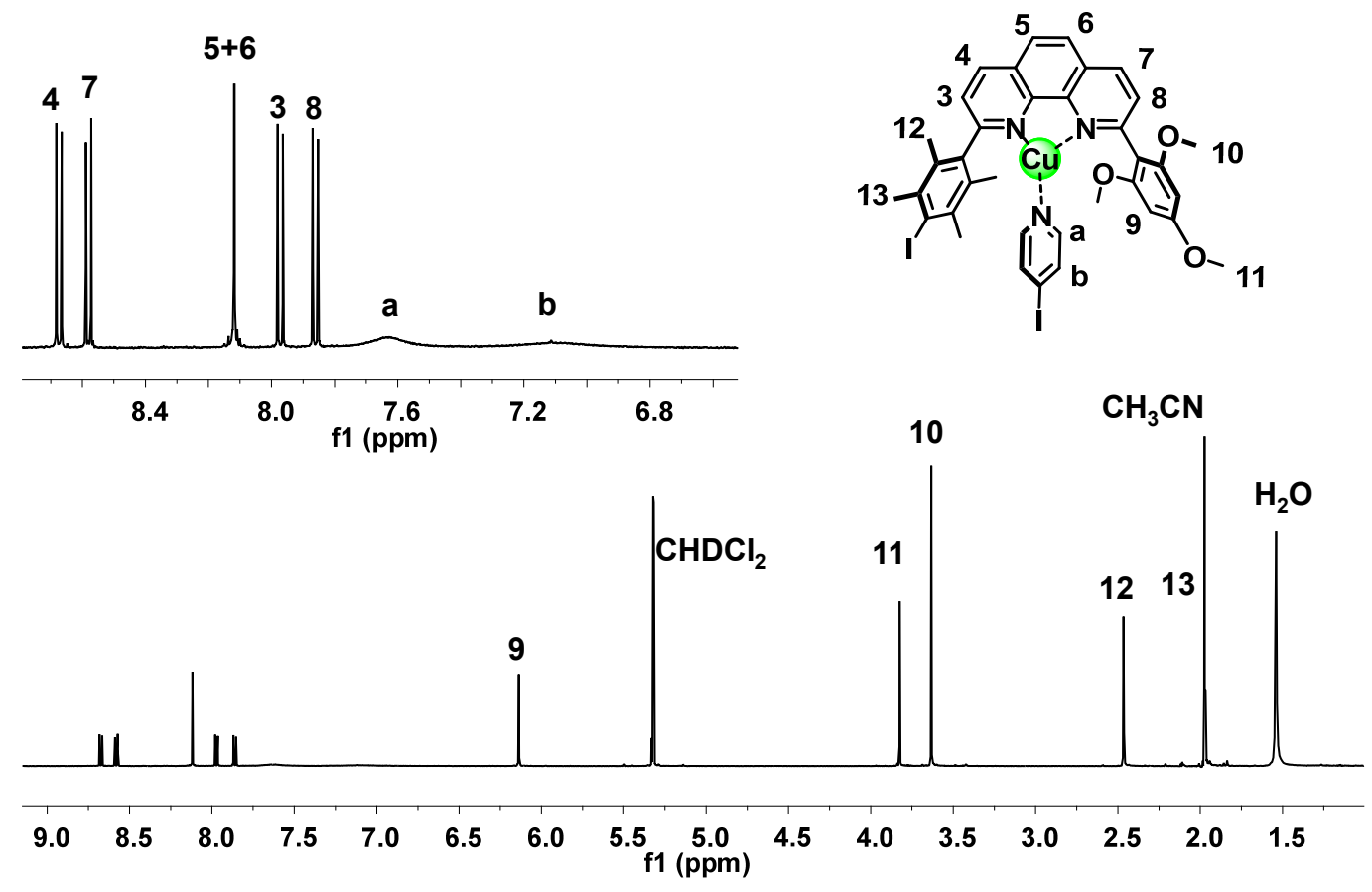

Figure S22. ${ }^{1} \mathrm{H}$ NMR spectrum of $[\mathrm{Cu}(\mathbf{2})(\mathbf{3})]^{+}$in $\mathrm{CD}_{2} \mathrm{Cl}_{2}(400 \mathrm{MHz}, 298 \mathrm{~K})$.

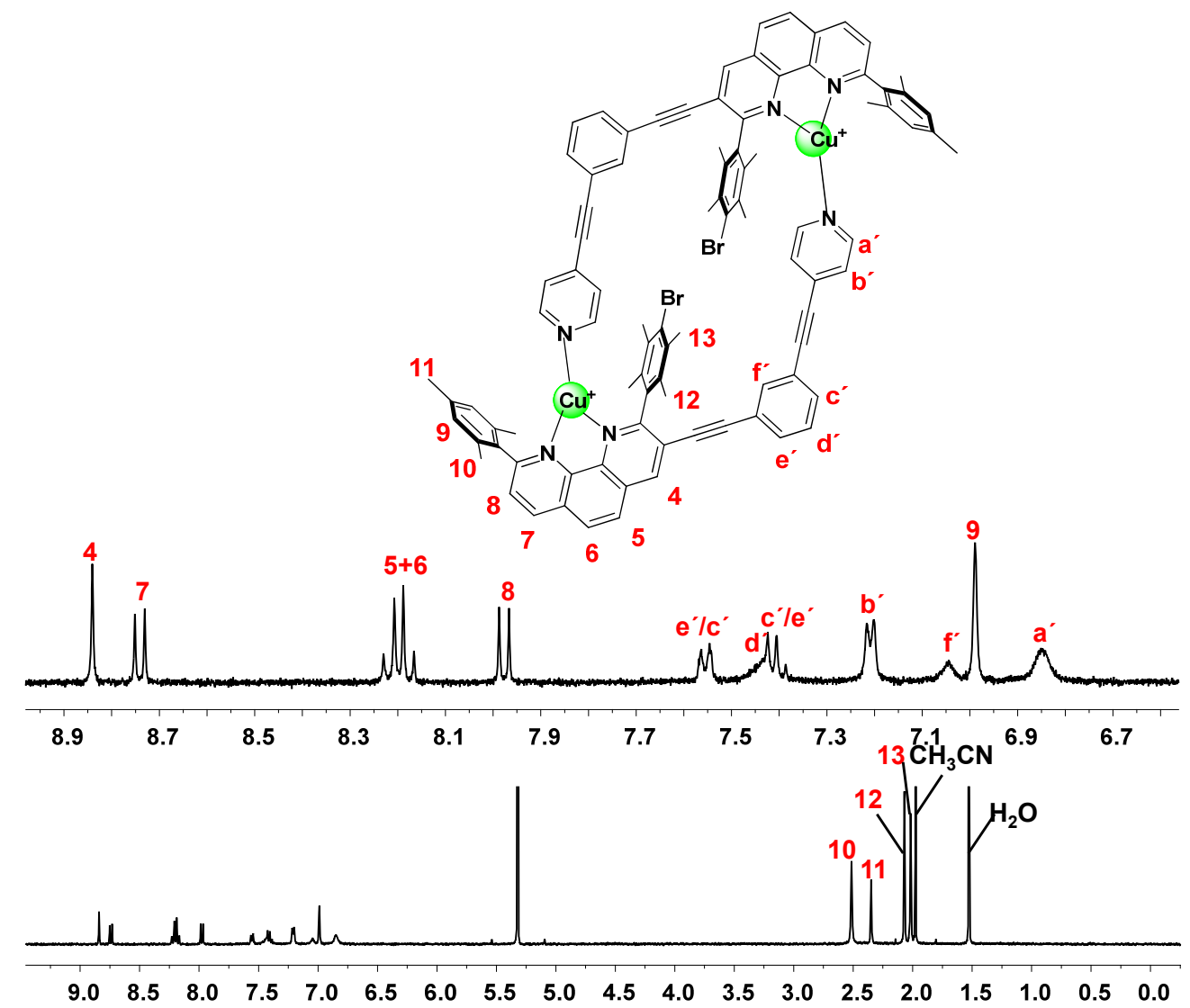

Figure S23. ${ }^{1} \mathrm{H}$ NMR spectrum of $\left[\mathrm{Cu}_{2}(\mathbf{R} 2)_{2}\right]^{2+}$ in $\mathrm{CD}_{2} \mathrm{Cl}_{2}(400 \mathrm{MHz}, 298 \mathrm{~K})$. 


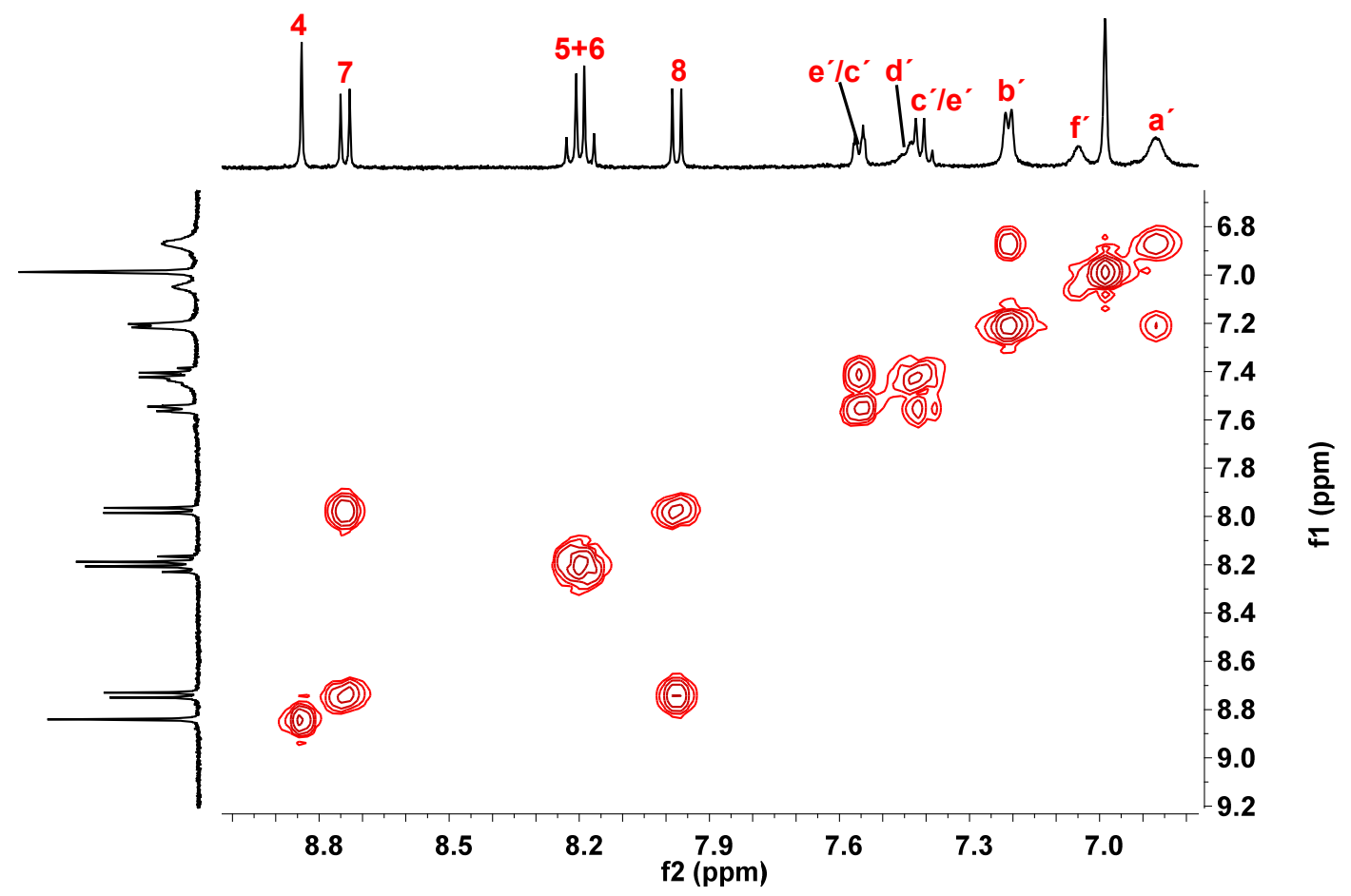

Figure S24. ${ }^{1} \mathrm{H}-{ }^{1} \mathrm{H}$ COSY spectrum of $\left[\mathrm{Cu}_{2}(\mathbf{R 2})_{2}\right]^{2+}$ in $\mathrm{CD}_{2} \mathrm{Cl}_{2}(400 \mathrm{MHz}, 298 \mathrm{~K})$.
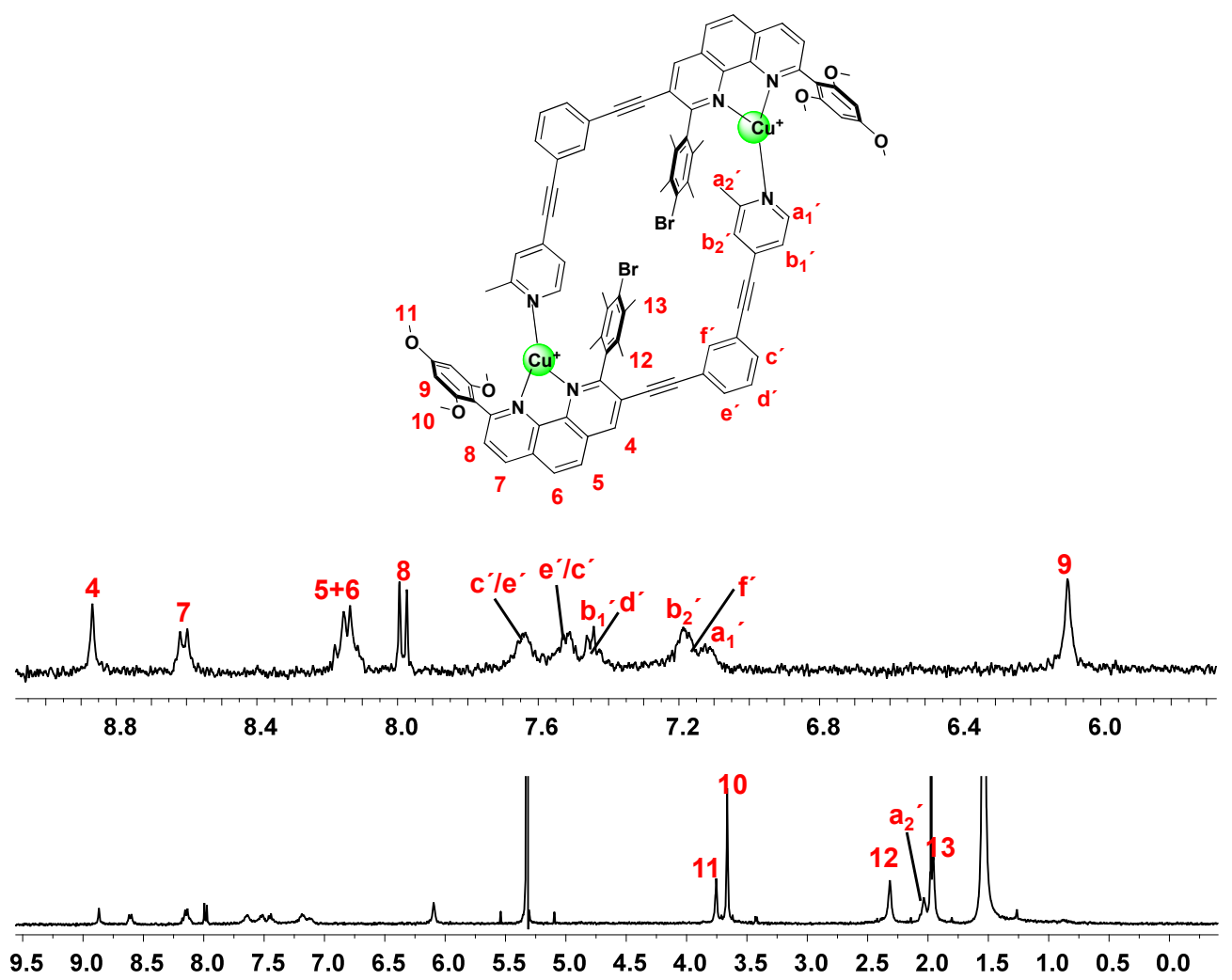

Figure S25. ${ }^{1} \mathrm{H}$ NMR spectrum of $\left[\mathrm{Cu}_{2}(\mathbf{R} 1)_{2}\right]^{2+}$ in $\mathrm{CD}_{2} \mathrm{Cl}_{2}(400 \mathrm{MHz}, 298 \mathrm{~K})$. 


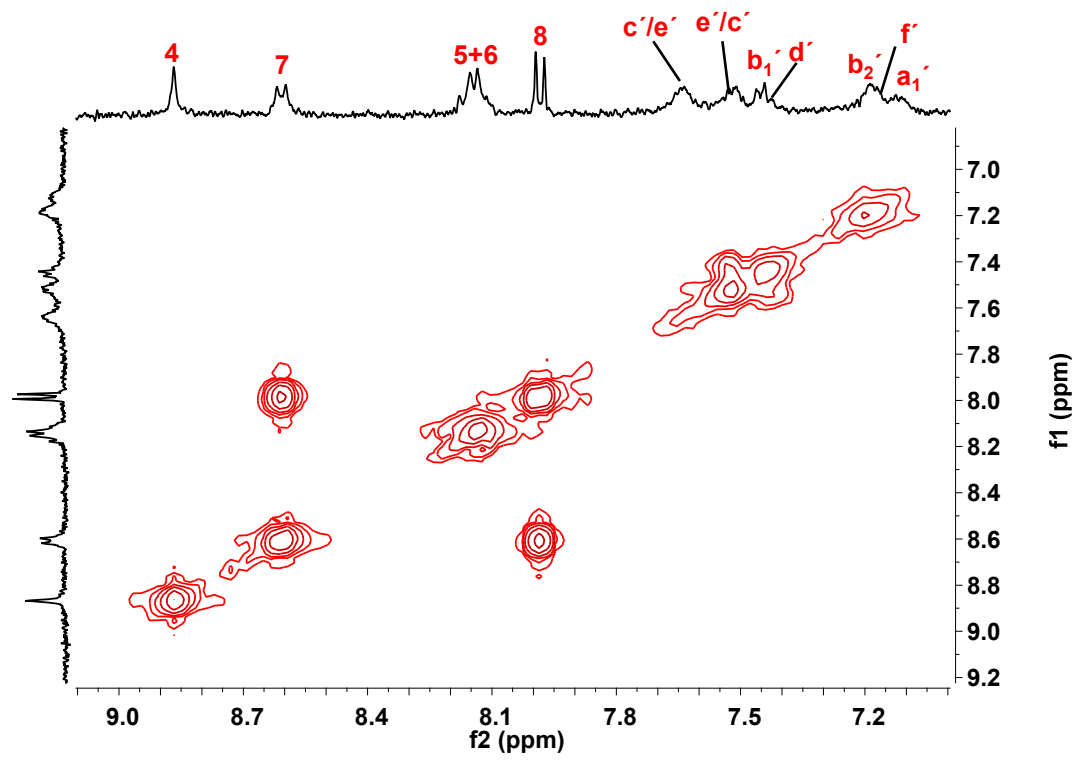

Figure S26. ${ }^{1} \mathrm{H}-{ }^{1} \mathrm{H}$ COSY spectrum of $\left[\mathrm{Cu}_{2}(\mathbf{R} 1)_{2}\right]^{2+}$ in $\mathrm{CD}_{2} \mathrm{Cl}_{2}(400 \mathrm{MHz}, 298 \mathrm{~K})$.

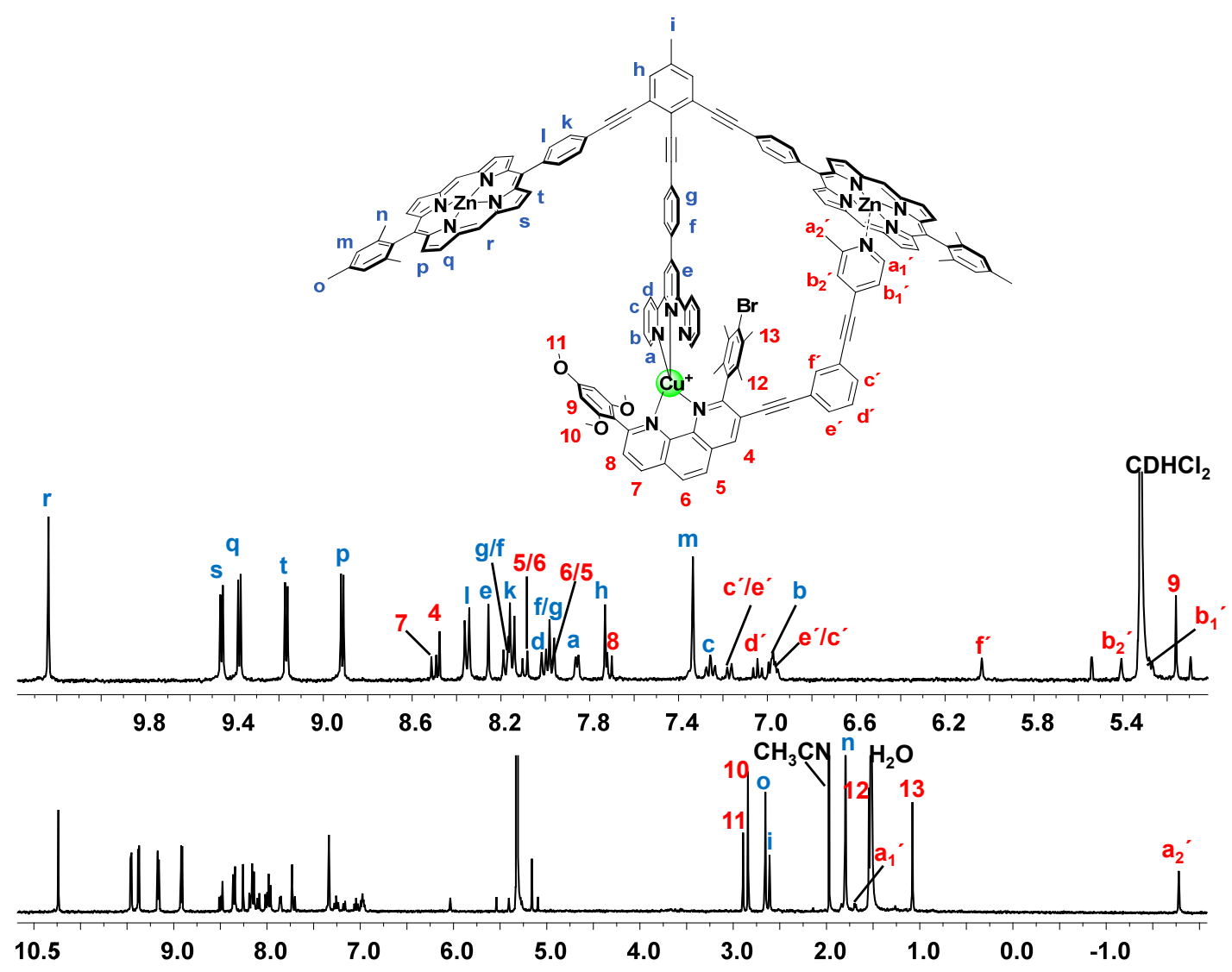

Figure S27. ${ }^{1} \mathrm{H}$ NMR spectrum of $[\mathrm{Cu}(\mathbf{S})(\mathbf{R} 1)]^{+}$in $\mathrm{CD}_{2} \mathrm{Cl}_{2}(400 \mathrm{MHz}, 298 \mathrm{~K})$. 


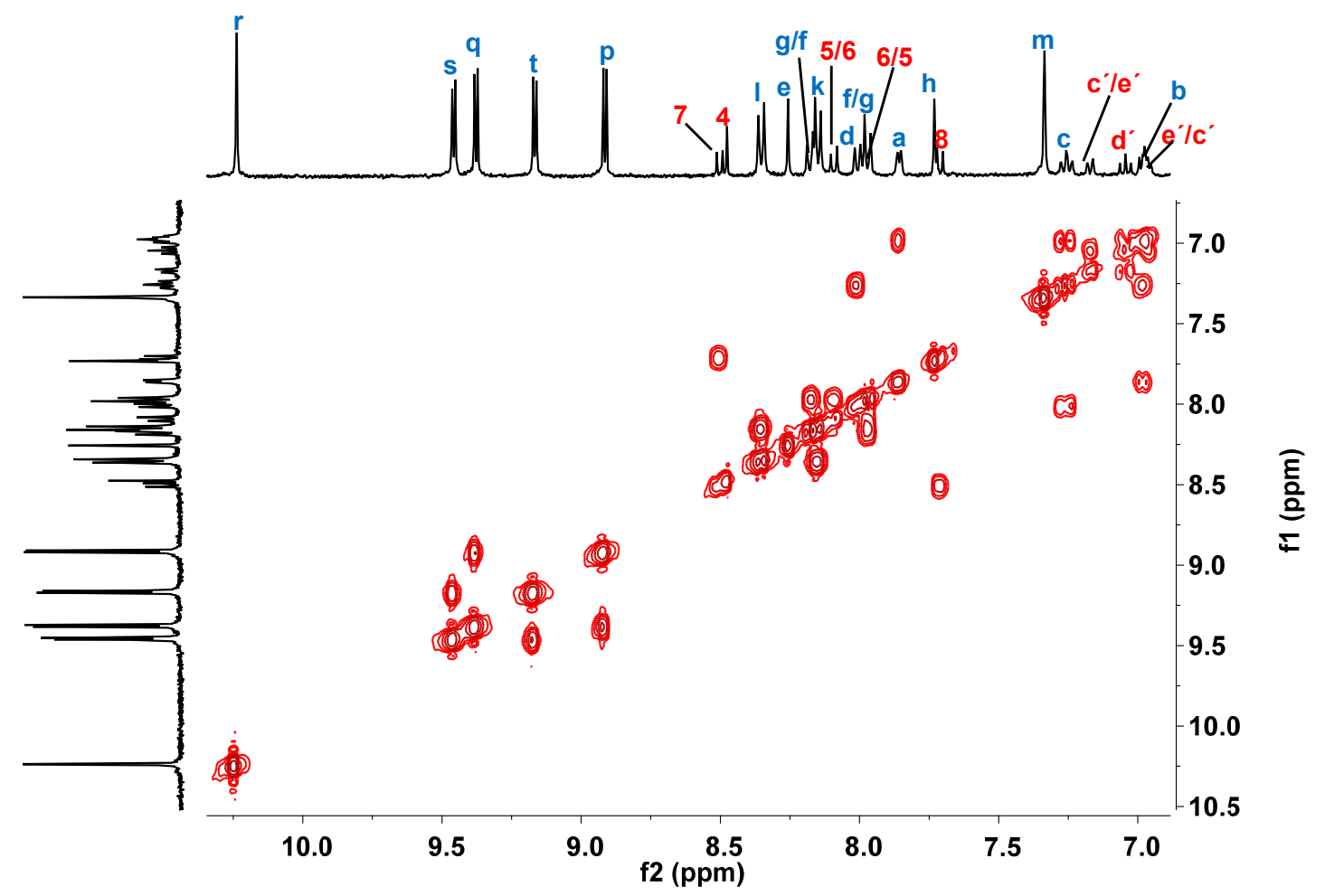

Figure S28. ${ }^{1} \mathrm{H}-{ }^{1} \mathrm{H}$ COSY spectrum of $[\mathrm{Cu}(\mathbf{S})(\mathbf{R} \mathbf{1})]^{+}$in $\mathrm{CD}_{2} \mathrm{Cl}_{2}(400 \mathrm{MHz}, 298 \mathrm{~K})$

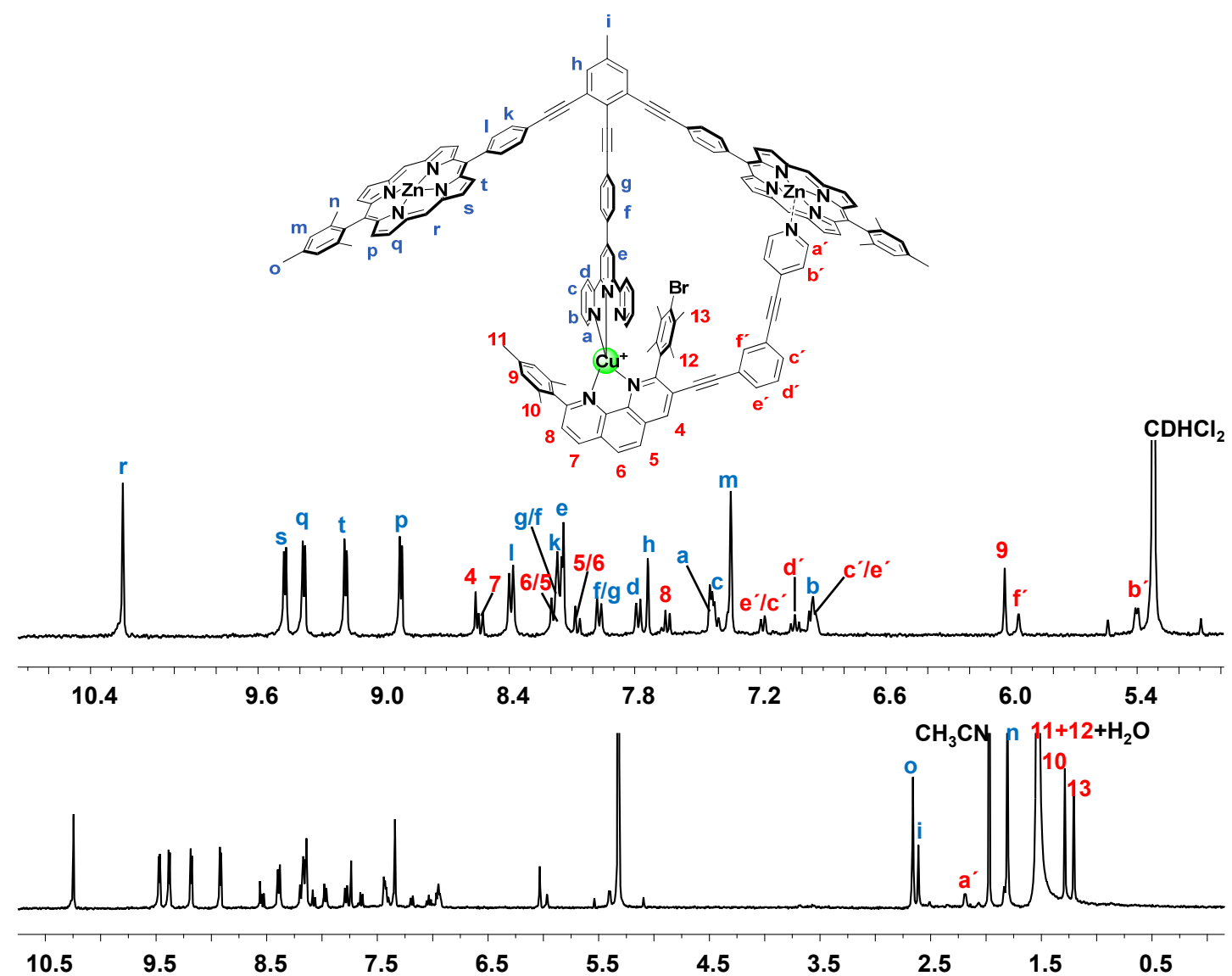

Figure S29. ${ }^{1} \mathrm{H}$ NMR spectrum of $[\mathrm{Cu}(\mathbf{S})(\mathbf{R 2})]^{+}$in $\mathrm{CD}_{2} \mathrm{Cl}_{2}(400 \mathrm{MHz}, 298 \mathrm{~K})$. 


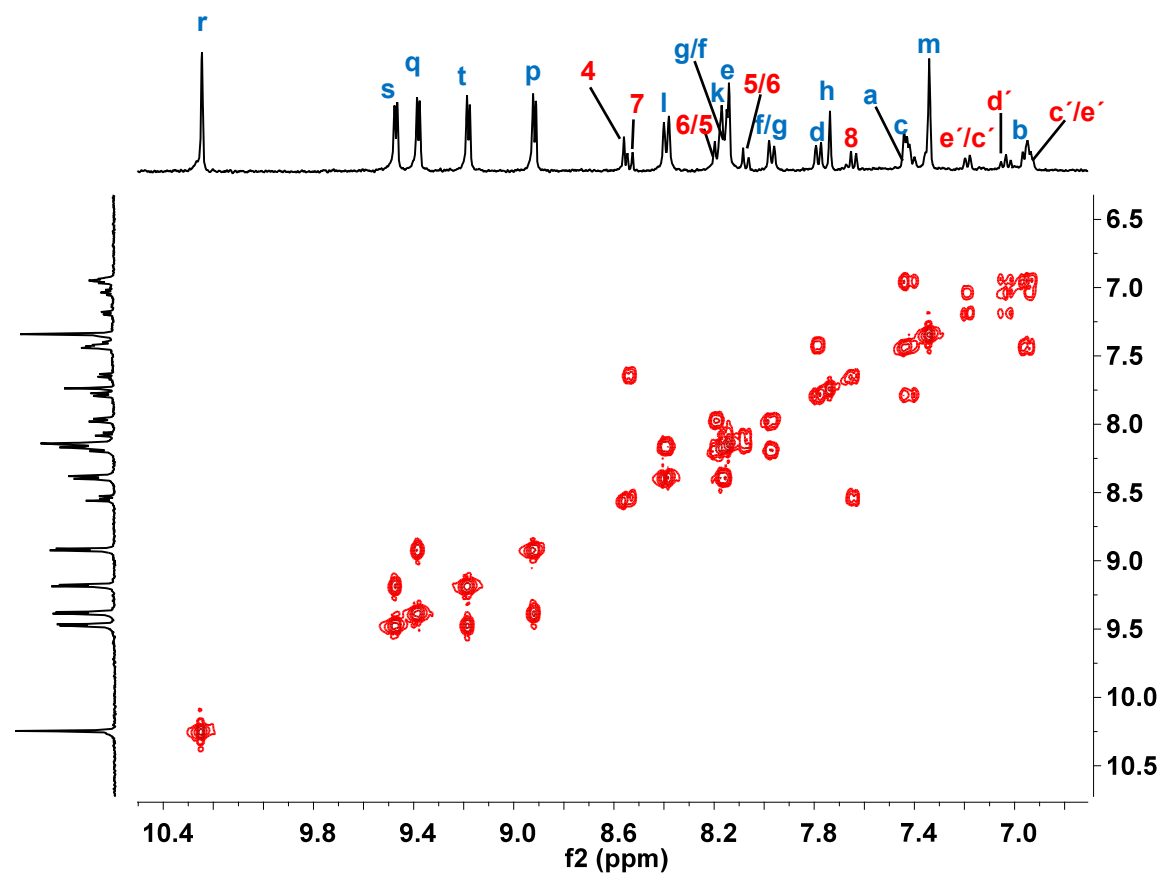

Figure S30. ${ }^{1} \mathrm{H}-{ }^{1} \mathrm{H}$ COSY spectrum of $[\mathrm{Cu}(\mathbf{S})(\mathbf{R 2})]^{+}$in $\mathrm{CD}_{2} \mathrm{Cl}_{2}(400 \mathrm{MHz}, 298 \mathrm{~K})$.

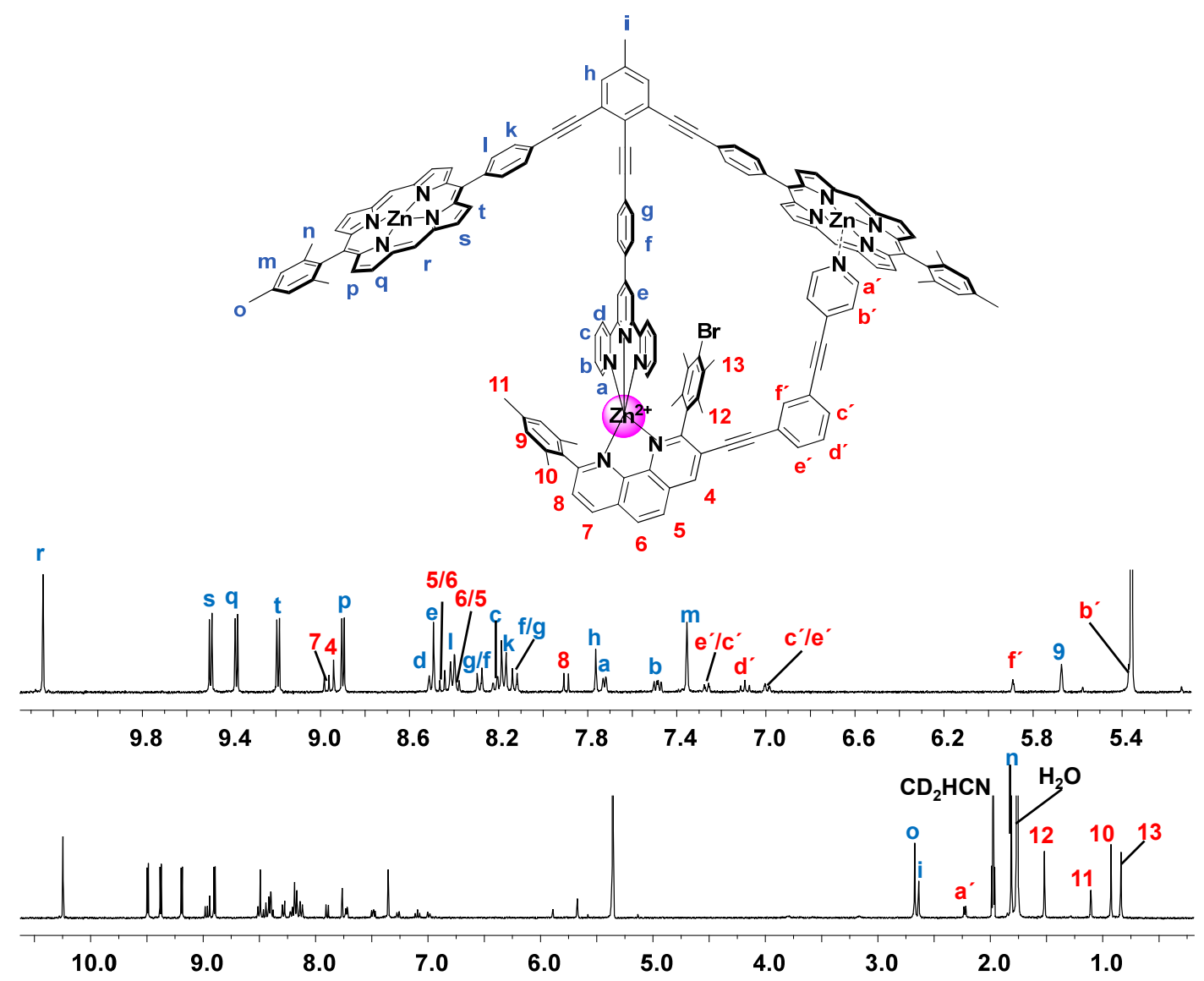

Figure S31. ${ }^{1} \mathrm{H}$ NMR spectrum of $[\mathrm{Zn}(\mathbf{S})(\mathbf{R 2})]^{2+}$ in $\mathrm{CD}_{2} \mathrm{Cl}_{2}: \mathrm{CD}_{3} \mathrm{CN}(5: 1)(400 \mathrm{MHz}, 298 \mathrm{~K})$. 


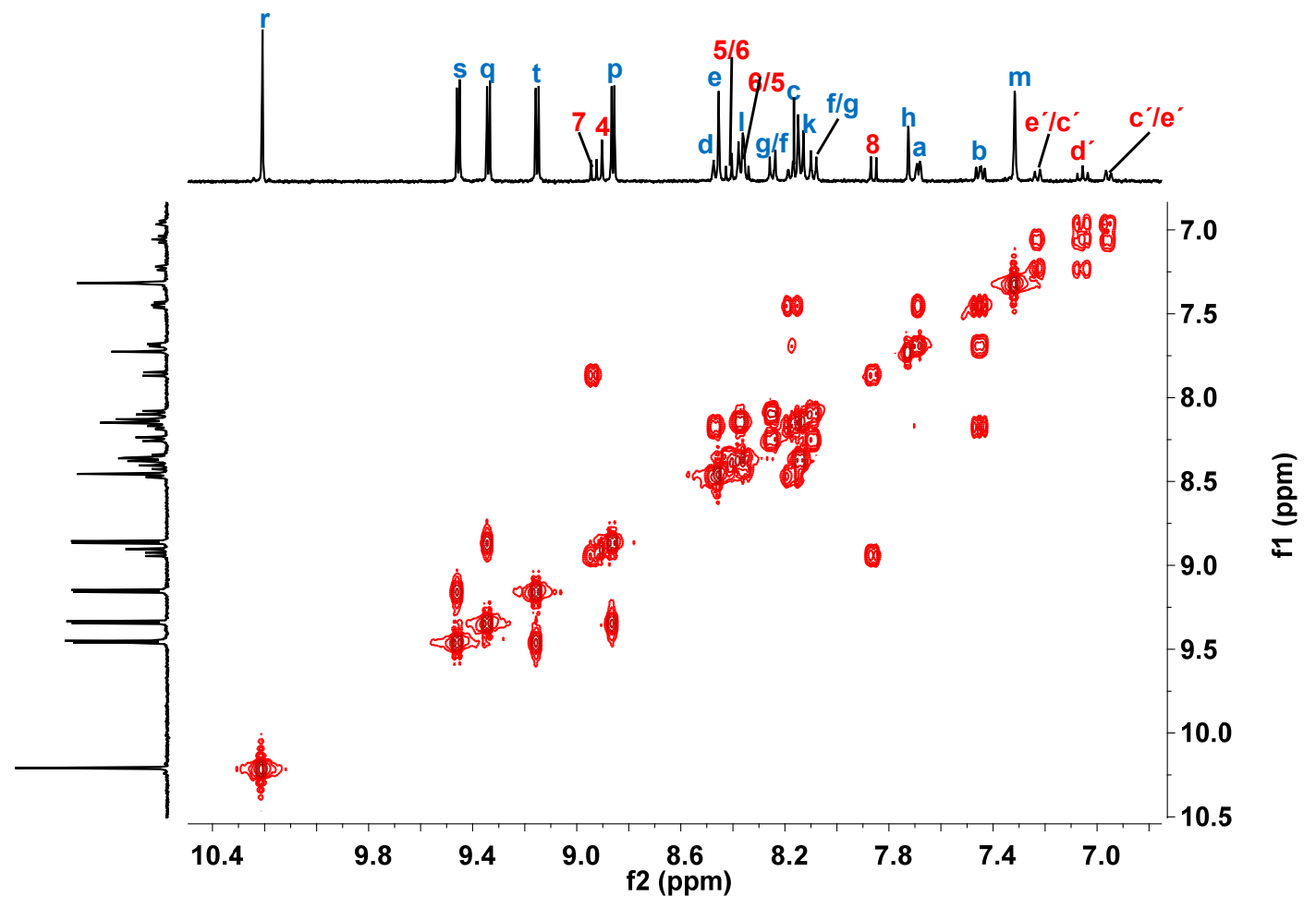

Figure S32. ${ }^{1} \mathrm{H}-{ }^{1} \mathrm{H}$ COSY NMR spectrum of $[\mathrm{Zn}(\mathbf{S})(\mathbf{R} 2)]^{2+}$ in $\mathrm{CD}_{2} \mathrm{Cl}_{2}: \mathrm{CD}_{3} \mathrm{CN}(5: 1)(400 \mathrm{MHz}, 298 \mathrm{~K})$.

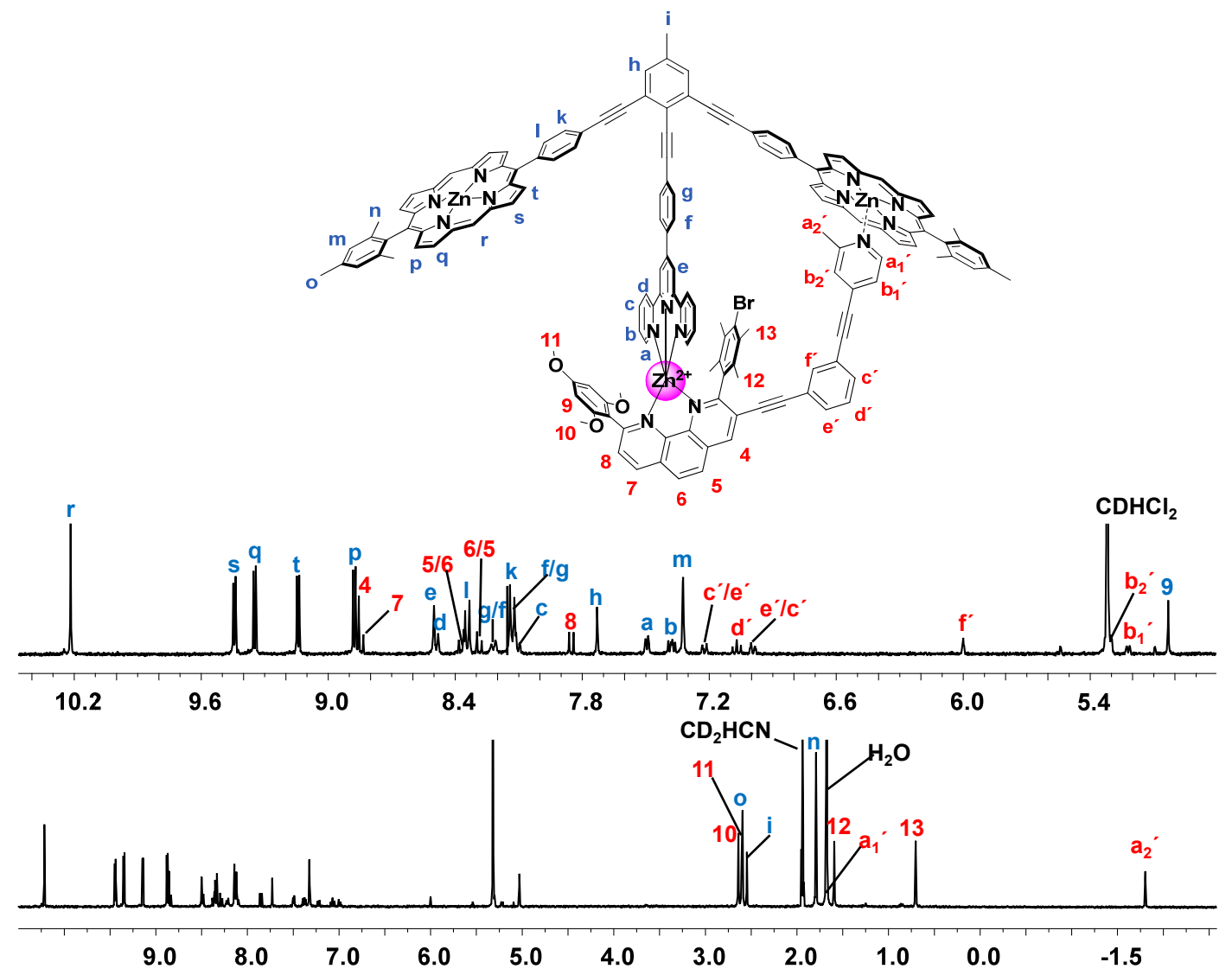

Figure S33. ${ }^{1} \mathrm{H}$ NMR spectrum of $[\mathrm{Zn}(\mathbf{S})(\mathbf{R} 1)]^{2+}$ in $\mathrm{CD}_{2} \mathrm{Cl}_{2}: \mathrm{CD}_{3} \mathrm{CN}(5: 1)(400 \mathrm{MHz}, 298 \mathrm{~K})$. 


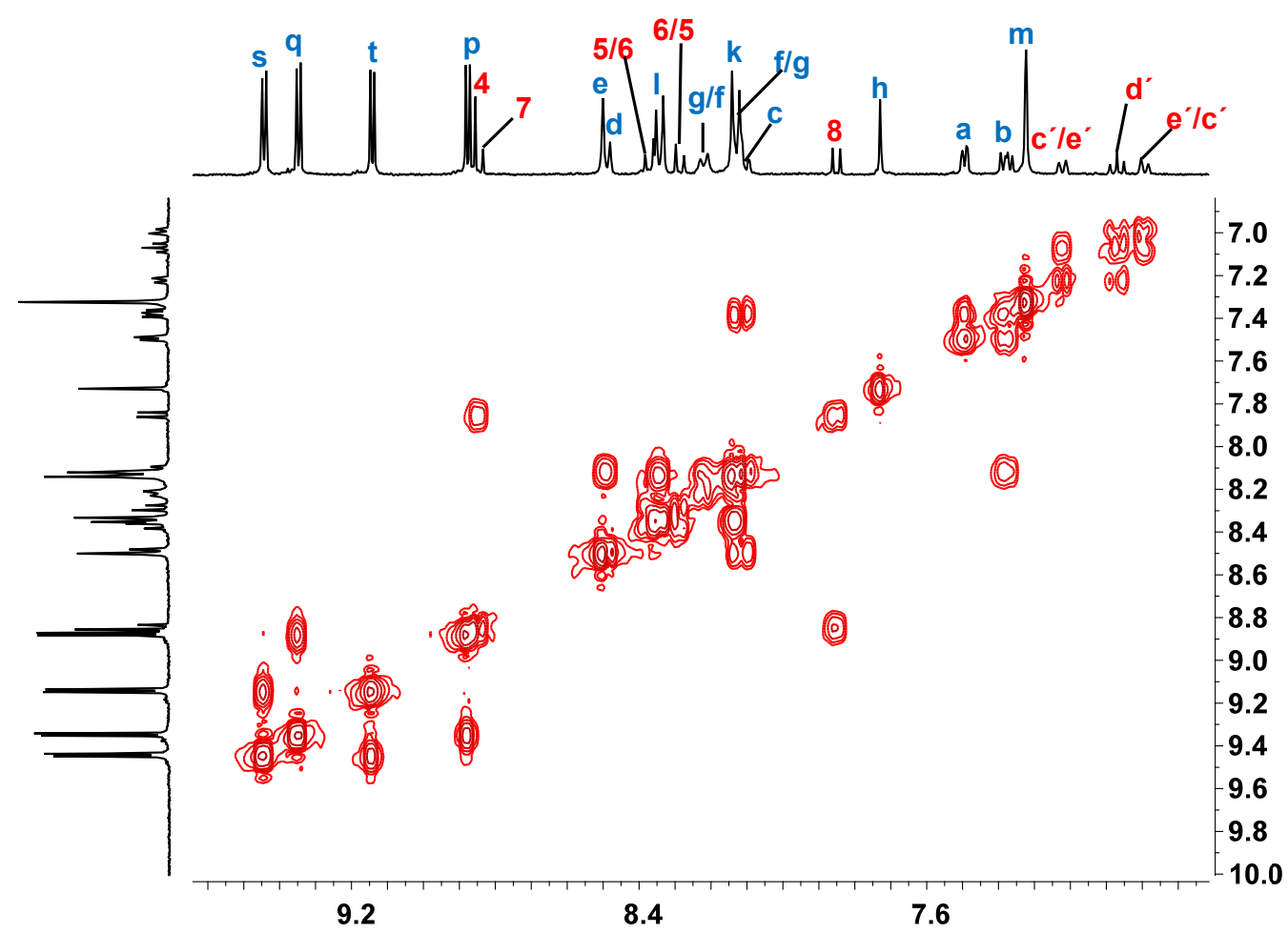

Figure S34. ${ }^{1} \mathrm{H}-{ }^{1} \mathrm{H}$ COSY NMR spectrum of $[\mathrm{Zn}(\mathbf{S})(\mathbf{R 1})]^{2+}$ in $\mathrm{CD}_{2} \mathrm{Cl}_{2}: \mathrm{CD}_{3} \mathrm{CN}(5: 1)(400 \mathrm{MHz}, 298 \mathrm{~K})$.

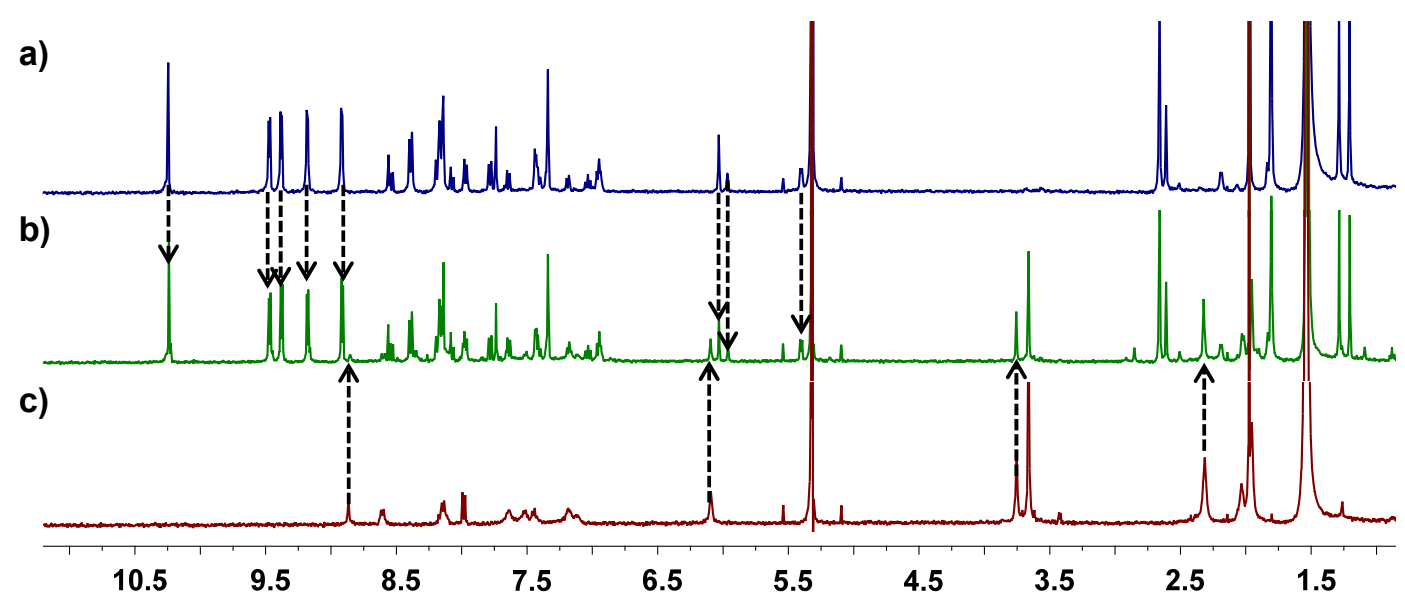

Figure S35. Partial ${ }^{1} \mathrm{H}$ NMR $\left(400 \mathrm{MHz}\right.$, in $\left.\mathrm{CD}_{2} \mathrm{Cl}_{2}\right)$ of (a) $[\mathrm{Cu}(\mathbf{S})(\mathbf{R 2})]^{+}$; (b) NetState $\mathbf{I}$ : mixture of $\left[\mathrm{Cu}_{2}(\mathbf{R} 1)_{2}\right]^{2+}$ and $[\mathrm{Cu}(\mathbf{S})(\mathbf{R 2})]^{+}$; (c) $\left[\mathrm{Cu}_{2}(\mathbf{R} 1)_{2}\right]^{2+}$. 


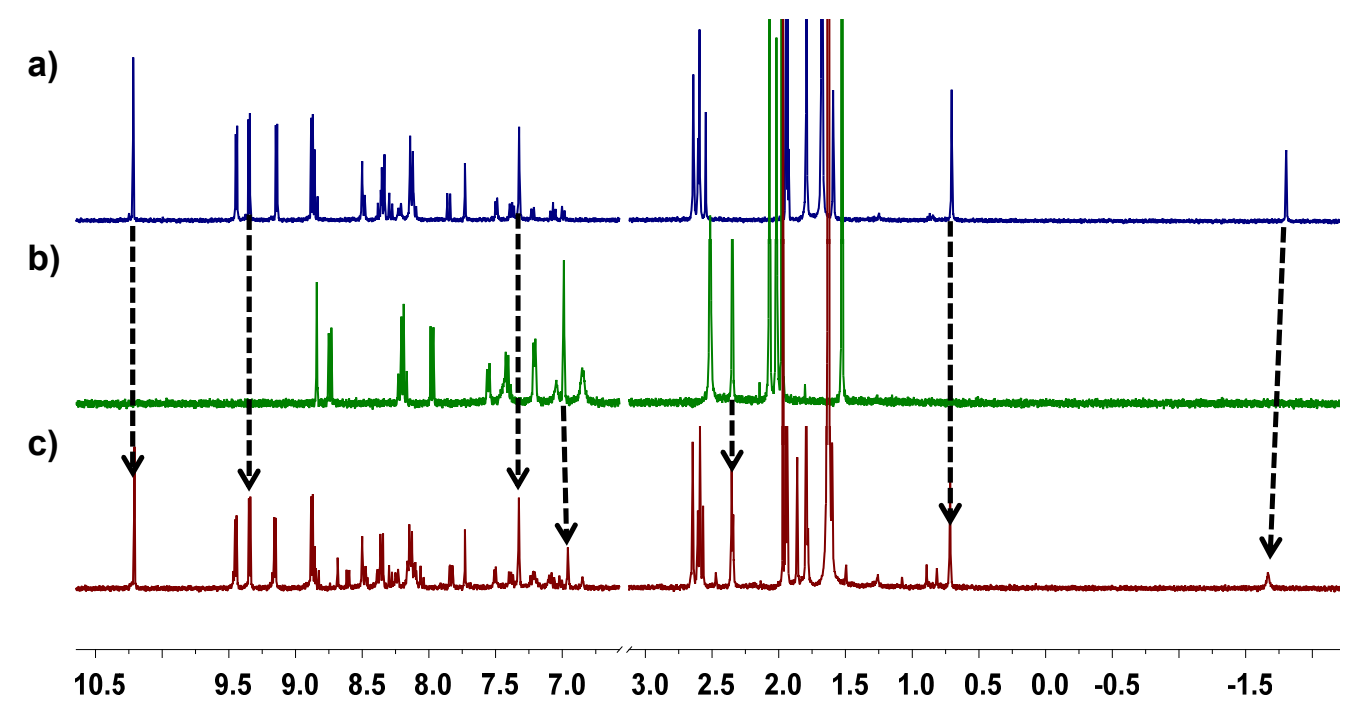

Figure S36. Partial ${ }^{1} \mathrm{H}$ NMR (400 MHz, $\left.\mathrm{CD}_{2} \mathrm{Cl}_{2}: \mathrm{CD}_{3} \mathrm{CN}(5: 1)\right)$ of (a) $[\mathrm{Zn}(\mathbf{S})(\mathbf{R 1})]^{2+}$; (b) $\left[\mathrm{Cu}_{2}(\mathbf{R 2})_{2}\right]^{2+}$; (c) NetState II: mixture of $\left[\mathrm{Cu}_{2}(\mathbf{R 2})_{2}\right]^{2+}$ and $[\mathrm{Zn}(\mathbf{S})(\mathbf{R} \mathbf{1})]^{2+}$.

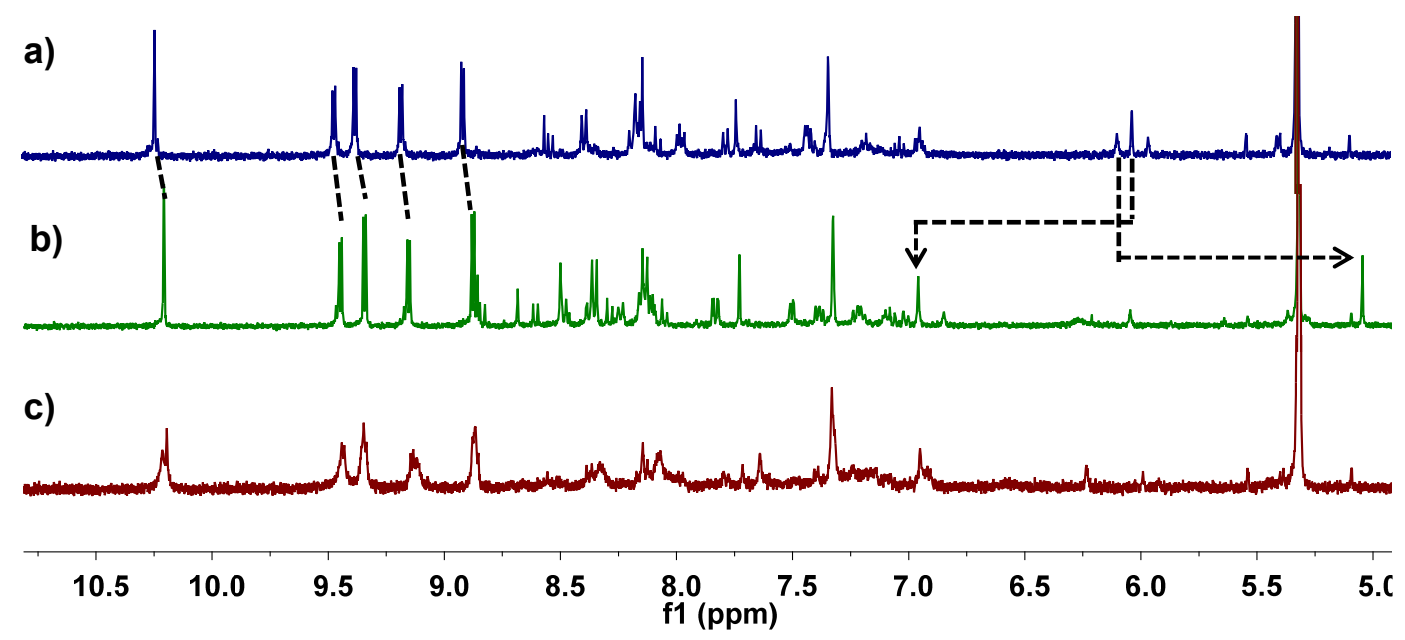

Figure S37. ${ }^{1} \mathrm{H}$ NMR spectra $\left(400 \mathrm{MHz}, \mathrm{CD}_{2} \mathrm{Cl}_{2}: \mathrm{CD}_{3} \mathrm{CN}=5: 1,298 \mathrm{~K}\right)$ showing the reversible switching between NetState I and II. The different NMR traces represent: (a) NetState I formed (= mixture of 0.5 equiv. of $\left[\mathrm{Cu}_{2}(\mathbf{R} 1)_{2}\right]^{2+}$ and 1.0 equiv. of $\left.[\mathrm{Cu}(\mathbf{S})(\mathbf{R 2})]^{+}\right)$after mixing of $\mathbf{S}, \mathbf{R} 1, \mathbf{R} 2$ and $\left[\mathrm{Cu}\left(\mathrm{CH}_{3} \mathrm{CN}\right)_{4}\right] \mathrm{PF}_{6}\left(1.69 \times 10^{-3} \mathrm{M}\right)$ in 1:1:1:1:2 ratio; (b) NetState II ( $=$ mixture of 0.5 equiv. of $\left[\mathrm{Cu}_{2}(\mathbf{R 2})_{2}\right]^{2+}$ and 1.0 equiv. of $[\mathrm{Zn}(\mathrm{S})(\mathbf{R} 1)]^{2+}$ ) is furnished after adding 1.0 equiv. of $\mathrm{Zn}(\mathrm{OTf})_{2}$ as a standard solution in $\mathrm{CD}_{3} \mathrm{CN}(36 \mu \mathrm{L})$ to (a); (c) NMR after addition of 1.0 equiv. of hexacyclen to NetState II. Reversibility (NetState II to NetState I) failed since the liberated copper(I) ions are unstable in solution. 

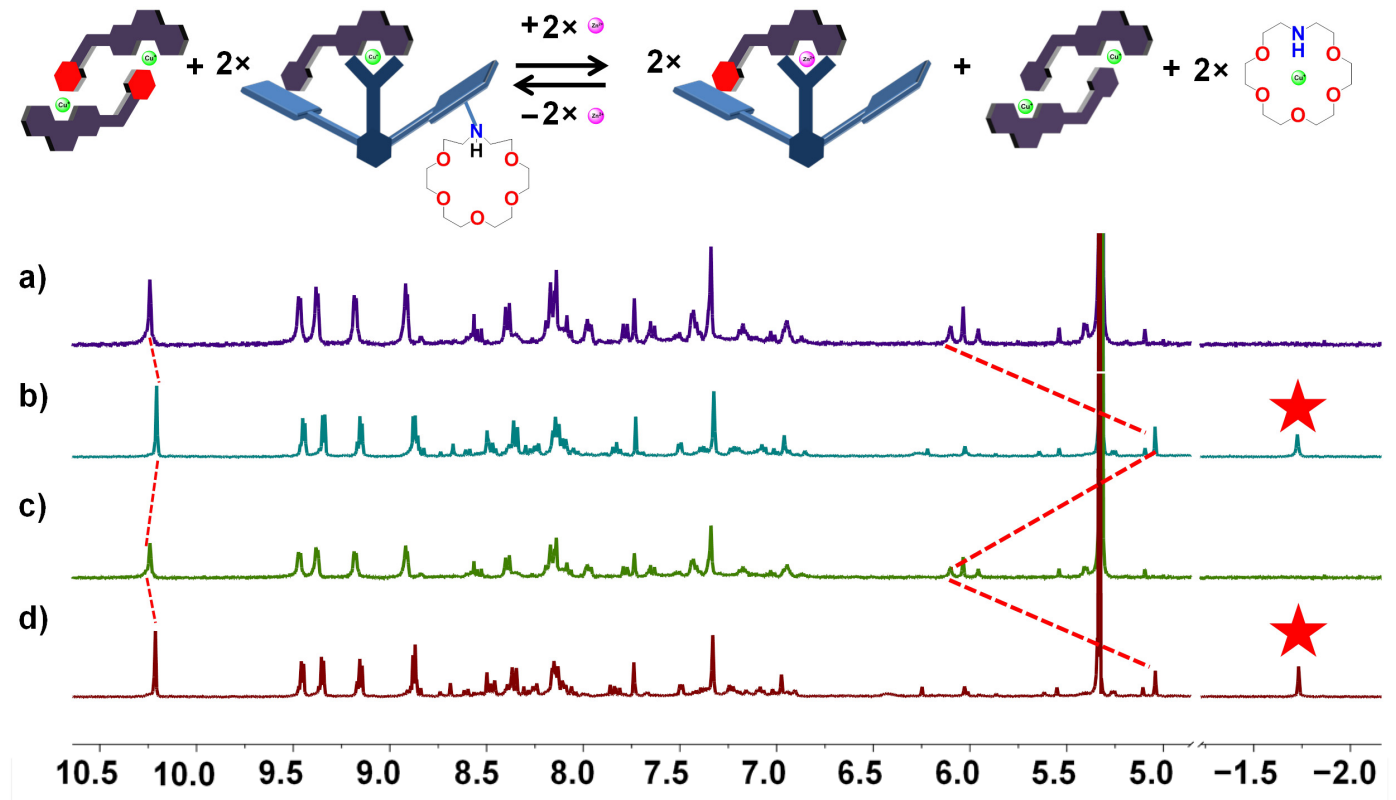

Figure S38. ${ }^{1} \mathrm{H}$ NMR spectra $\left(400 \mathrm{MHz}, \mathrm{CD}_{2} \mathrm{Cl}_{2}: \mathrm{CD}_{3} \mathrm{CN}(5: 1), 298 \mathrm{~K}\right)$ showing the reversible switching between NetState I and II in presence of a receptor 7. The different NMR traces represent: (a) NetState I (= mixture of 0.5 equiv. of $\left[\mathrm{Cu}_{2}(\mathbf{R} 1)_{2}\right]^{2+}$ and 1.0 equiv. of $\left.[\mathrm{Cu}(\mathbf{S})(\mathbf{R 2})]^{+}\right)$obtained after mixing of $\mathbf{S}, \mathbf{R 1}, \mathbf{R 2}, 7$ and $\left[\mathrm{Cu}\left(\mathrm{CH}_{3} \mathrm{CN}\right)_{4}\right] \mathrm{PF}_{6}(1.67 \times$ $\left.10^{-3} \mathrm{M}\right)$ in 1:1:1:1:2 ratio; (b) NetState II (= mixture of 0.5 equiv. of $\left[\mathrm{Cu}_{2}(\mathbf{R 2})_{2}\right]^{2+}$ and 1.0 equiv. of $\left.[\mathrm{Zn}(\mathbf{S})(\mathbf{R} 1)]^{2+}\right)$ furnished after adding 1.0 equiv. of $\mathrm{Zn}(\mathrm{OTf})_{2}$ as a standard solution in $\mathrm{CD}_{3} \mathrm{CN}(47 \mu \mathrm{L})$ to (a); (c) NMR of NetState I received after addition of 1.0 equiv. of hexacyclen; (d) NetState II after adding another 1.0 equiv. of $\mathrm{Zn}(\mathrm{OTf})_{2}$. Red asterisk marked signals arise from the $\alpha$-methyl group of $\mathbf{R} 1$ in nanorotor $[\operatorname{Zn}(\mathbf{S})(\mathbf{R} 1)]^{2+}$, indicating the formation of NetState II. 


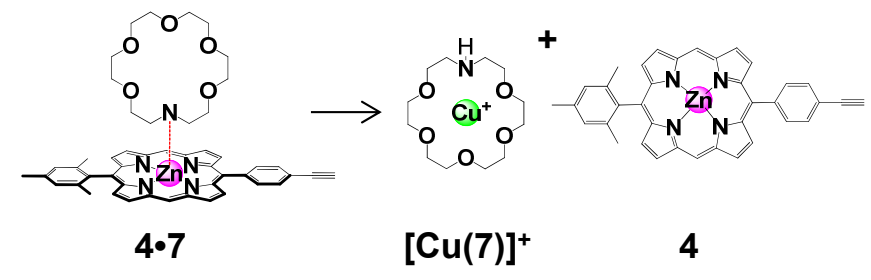

a)

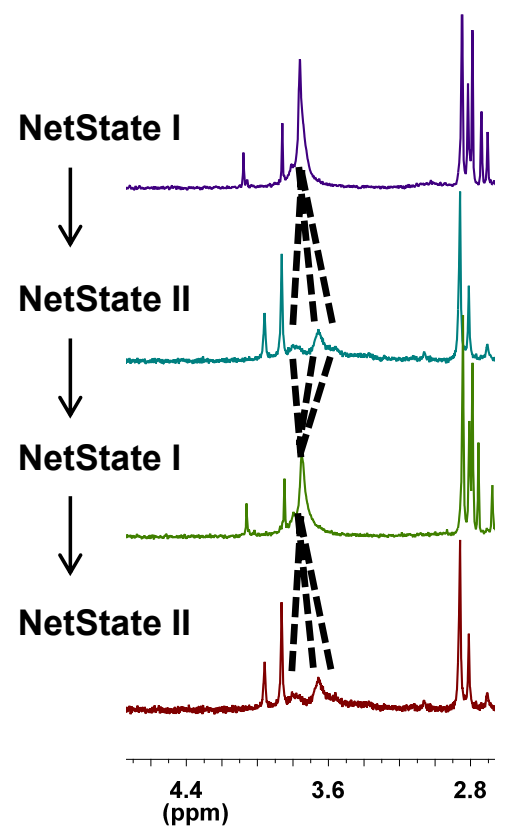

b)

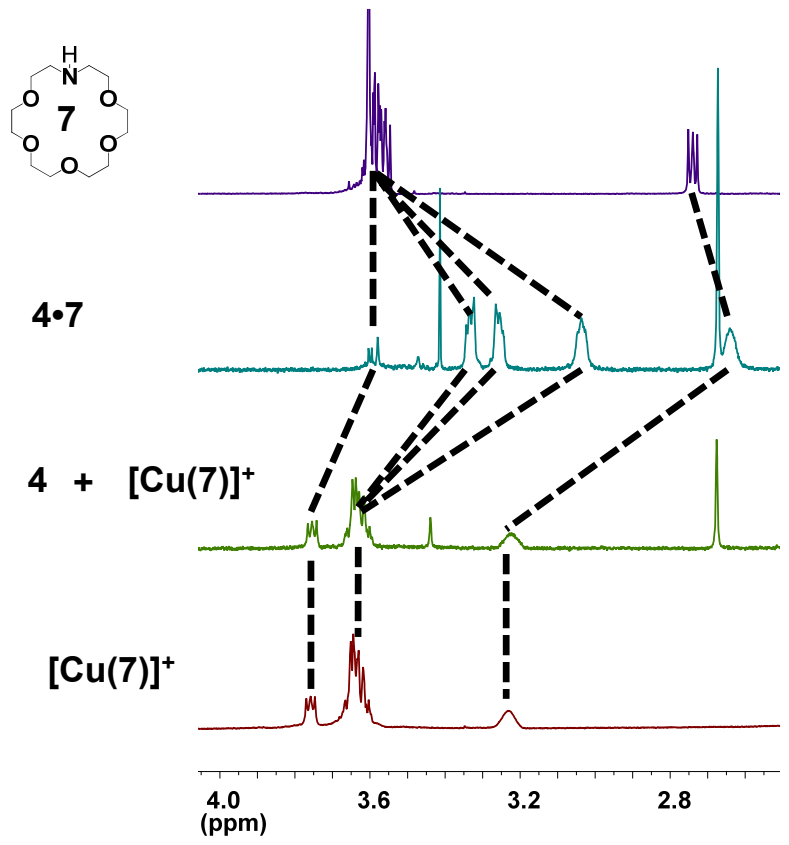

Figure S39. Changes in the ${ }^{1} \mathrm{H}$ NMR (400 MHz, $298 \mathrm{~K}$ ) (a) during switching between NetState I and NetState II (see Figure S38) showing the capture and release of $\mathrm{Cu}^{+}$by receptor 7. (b) ${ }^{1} \mathrm{H}$ NMR signatures (from top to bottom) of 7; of $[(4) \cdot(7)]$, of mixture of 4 and $[\mathrm{Cu}(7)]^{+}$(obtained upon addition of 1.0 equiv of $\left[\mathrm{Cu}\left(\mathrm{CH}_{3} \mathrm{CN}\right)_{4} \mathrm{PF}_{6}\right.$ to $\left.[(4) \cdot(7)]\right)$, and of $[\mathrm{Cu}(7)]^{+}$in $\mathrm{CD}_{2} \mathrm{Cl}_{2}$. 


\section{DOSY NMR spectra}

\section{Calculation of hydrodynamic radius from:}

a) DOSY: The diffusion coefficient $D$ for $[\mathrm{Zn}(\mathbf{S})(\mathbf{R})]^{2+}$ and $[\mathrm{Cu}(\mathbf{S})(\mathbf{R})]^{+}$was obtained from their DOSY spectrum. The corresponding hydrodynamic radius was calculated by using the Stokes-Einstein equation

$$
r=k_{B} T / 6 \pi \eta D
$$

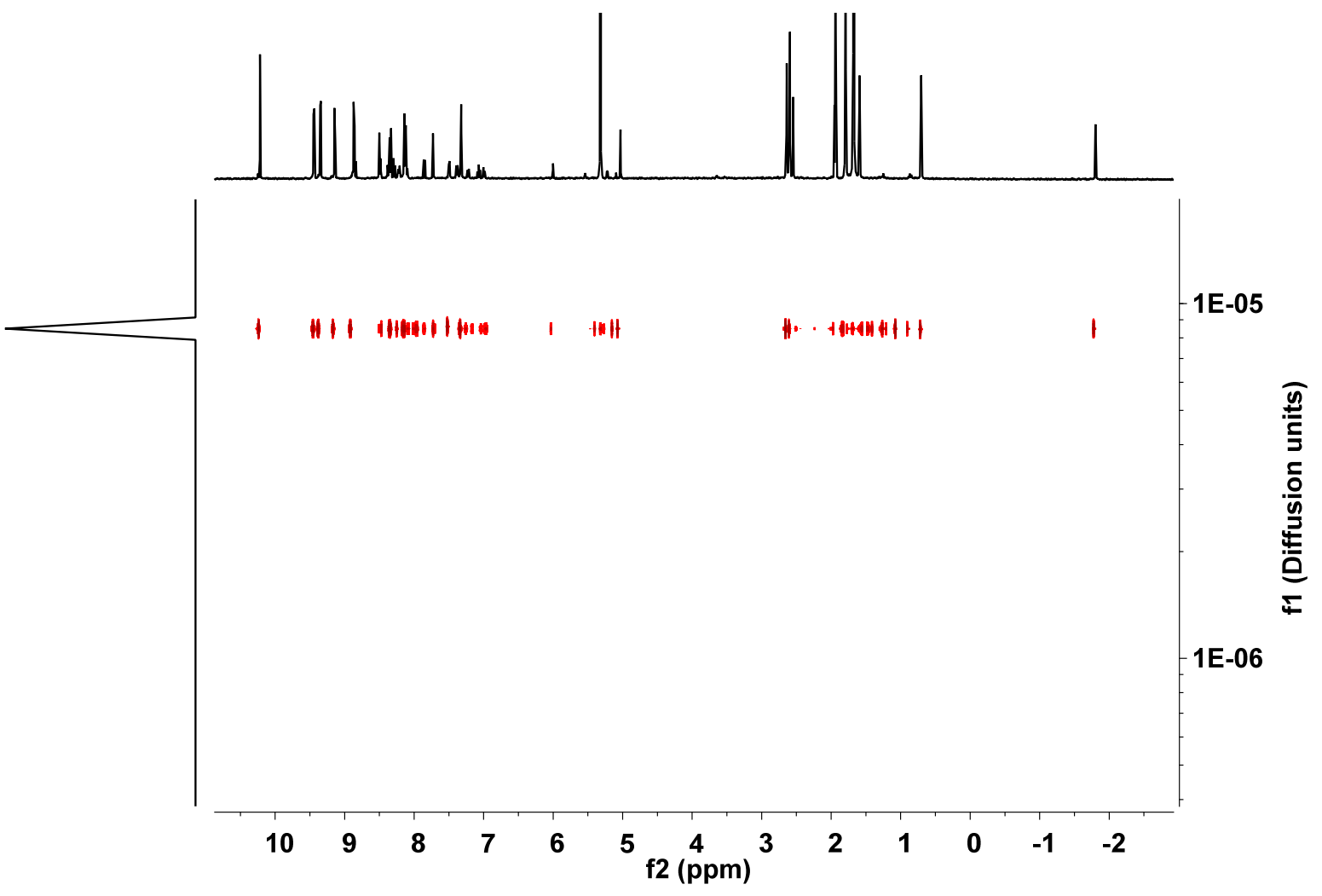

Figure S40. ${ }^{1} \mathrm{H}-\mathrm{DOSY}$ NMR of $[\mathrm{Zn}(\mathbf{S})(\mathbf{R 1})]^{2+}$ in $\mathrm{CD}_{2} \mathrm{Cl}_{2}: \mathrm{CD} 3 \mathrm{CN}(5: 1)(600 \mathrm{MHz}, 298 \mathrm{~K})$. Diffusion coefficient $D=$ $5.32 \times 10^{-10} \mathrm{~m}^{2} \mathrm{~s}^{-1}$, hydrodynamic radius $r=9.9 \AA$. 


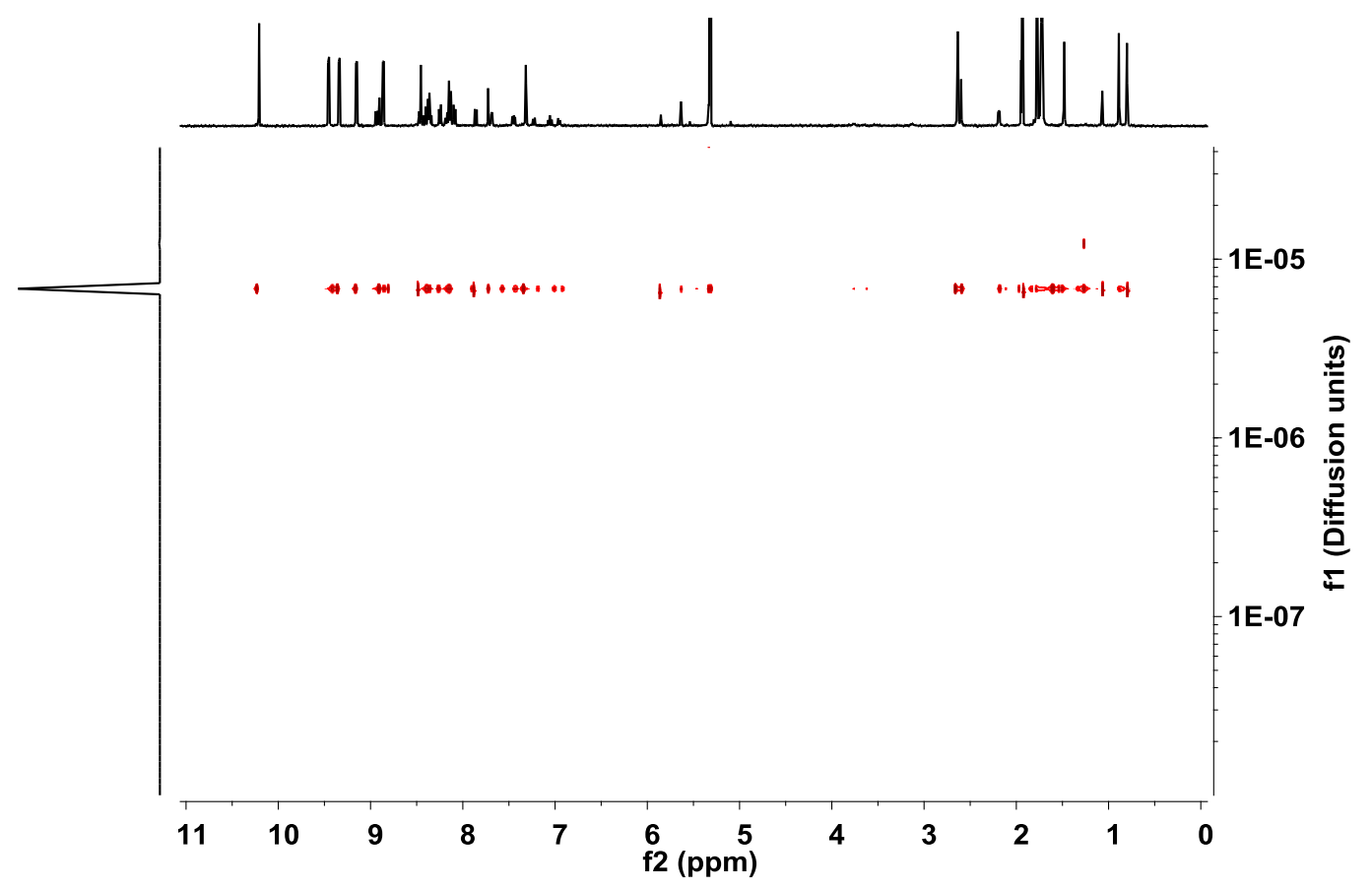

Figure S41. ${ }^{1} \mathrm{H}-\mathrm{DOSY}$ NMR of $[\mathrm{Zn}(\mathbf{S})(\mathbf{R 2})]^{2+}$ in $\mathrm{CD}_{2} \mathrm{Cl}_{2}: \mathrm{CD} 3 \mathrm{CN}(5: 1)(600 \mathrm{MHz}, 298 \mathrm{~K})$. Diffusion coefficient $D=$ $4.76 \times 10^{-10} \mathrm{~m}^{2} \mathrm{~s}^{-1}$, hydrodynamic radius $r=11.1 \AA$.

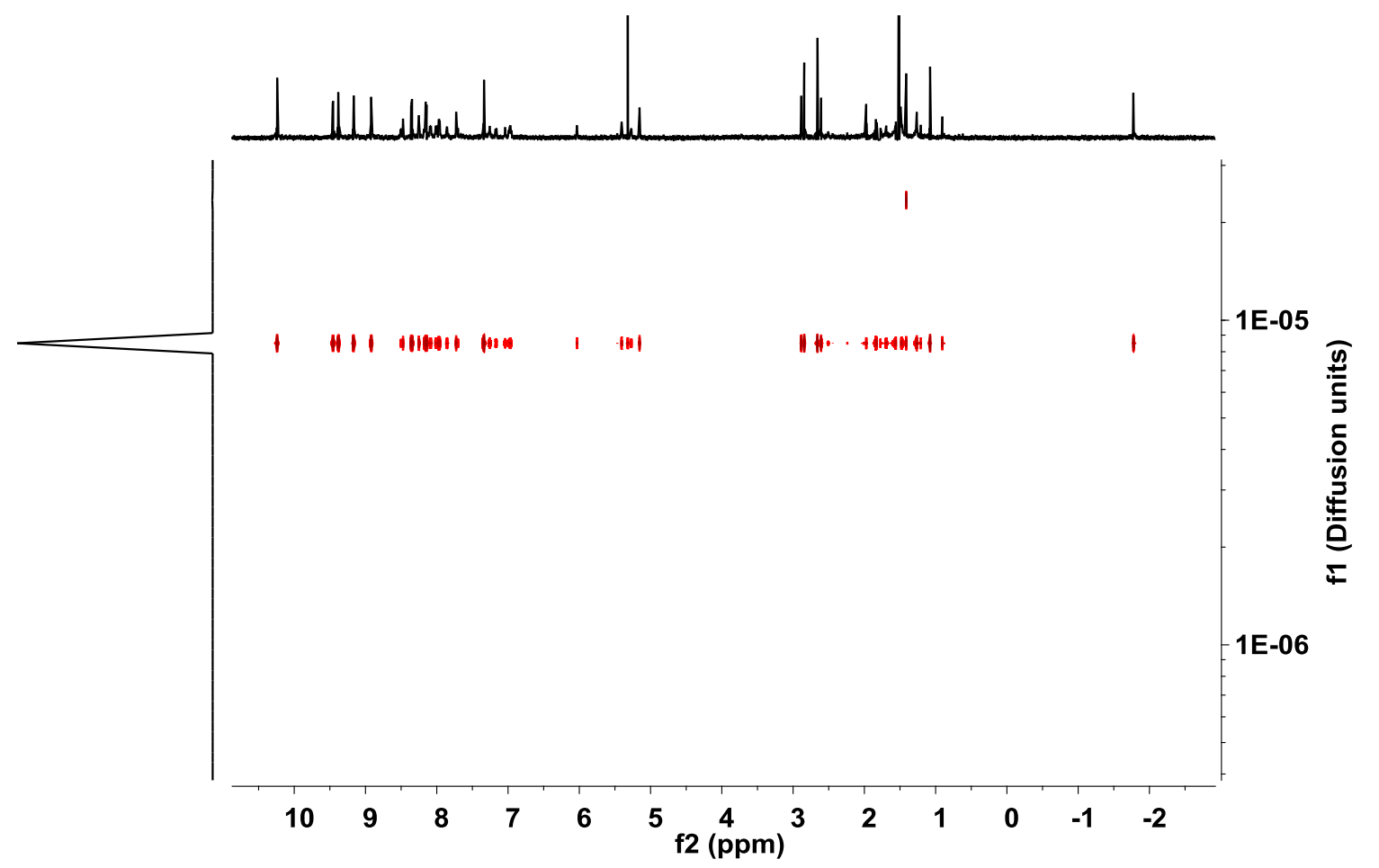

Figure S42. ${ }^{1} \mathrm{H}-\mathrm{DOSY}$ NMR of $[\mathrm{Cu}(\mathbf{S})(\mathbf{R} \mathbf{1})]^{+}$in $\mathrm{CD}_{2} \mathrm{Cl}_{2}(600 \mathrm{MHz}, 298 \mathrm{~K})$. Diffusion coefficient $D=5.92 \times 10^{-10} \mathrm{~m}^{2}$ $\mathrm{s}^{-1}$, hydrodynamic radius $r=8.9 \AA$. 


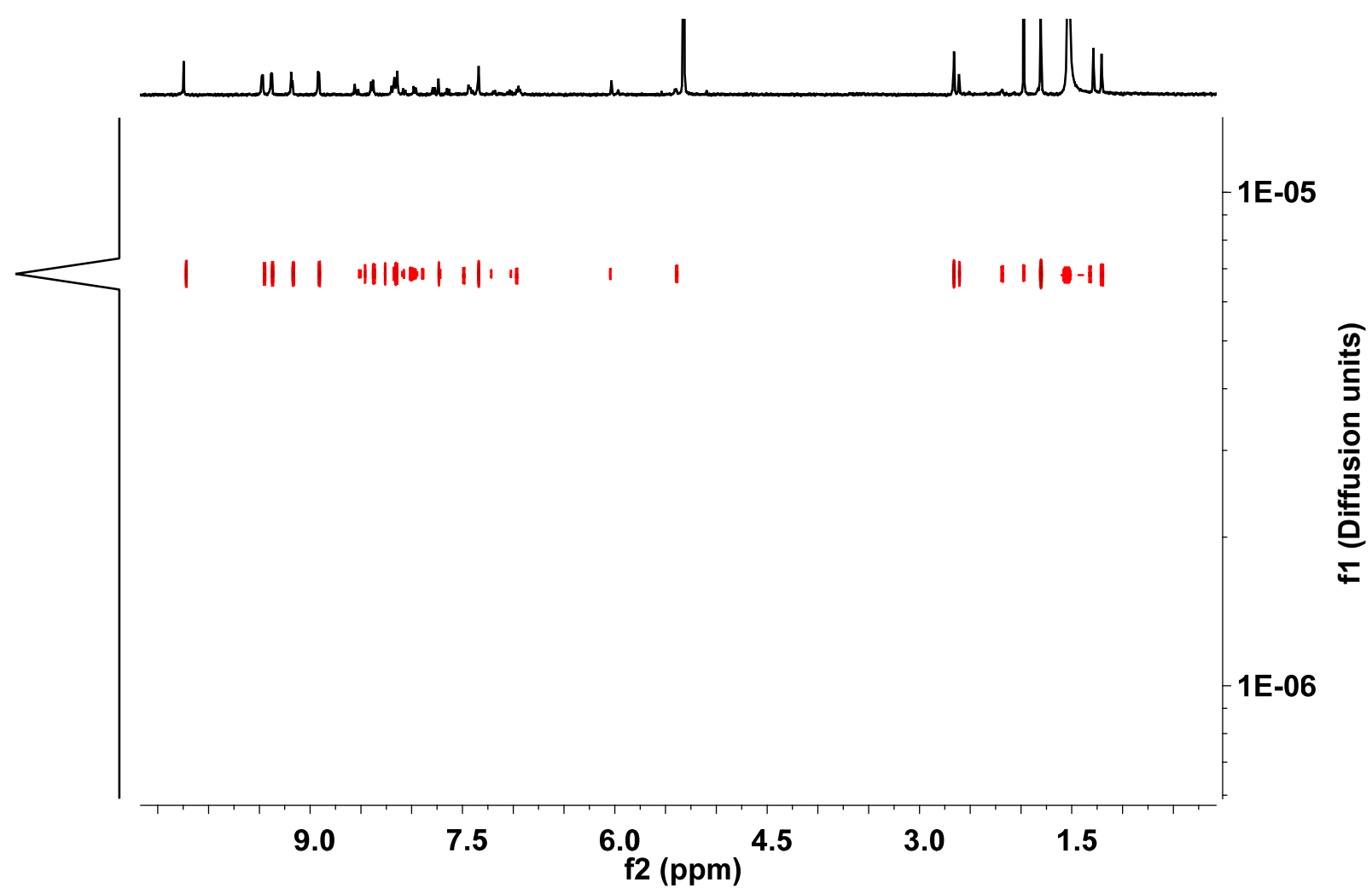

Figure S43. ${ }^{1} \mathrm{H}-\mathrm{DOSY}$ NMR of $[\mathrm{Cu}(\mathbf{S})(\mathbf{R} 2)]^{+}$in $\mathrm{CD}_{2} \mathrm{Cl}_{2}(600 \mathrm{MHz}, 298 \mathrm{~K})$. Diffusion coefficient $D=4.87 \times 10^{-10} \mathrm{~m}^{2}$ $\mathrm{s}^{-1}$, hydrodynamic radius $r=10.9 \AA$.

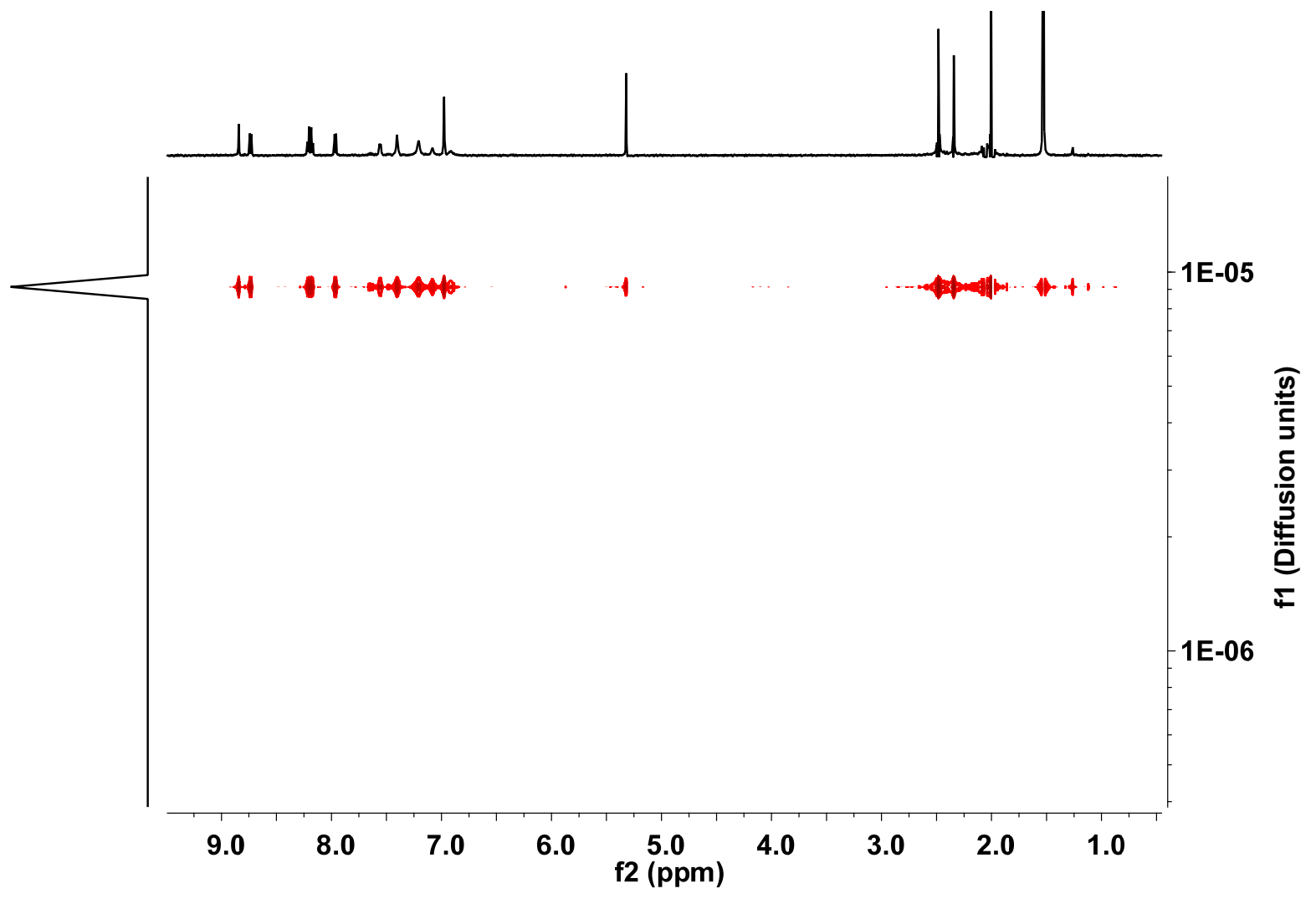

Figure S44. ${ }^{1} \mathrm{H}-\mathrm{DOSY} \mathrm{NMR}$ of $\left[\mathrm{Cu}_{2}(\mathbf{R 2})_{2}\right]^{2+}$ in $\mathrm{CD}_{2} \mathrm{Cl}_{2}(600 \mathrm{MHz}, 298 \mathrm{~K})$. Diffusion coefficient $D=6.78 \times 10^{-10} \mathrm{~m}^{2} \mathrm{~s}^{-}$ ${ }^{1}$, hydrodynamic radius $r=7.8 \AA$. 


\section{Variable temperature studies and determination of kinetic parameters}

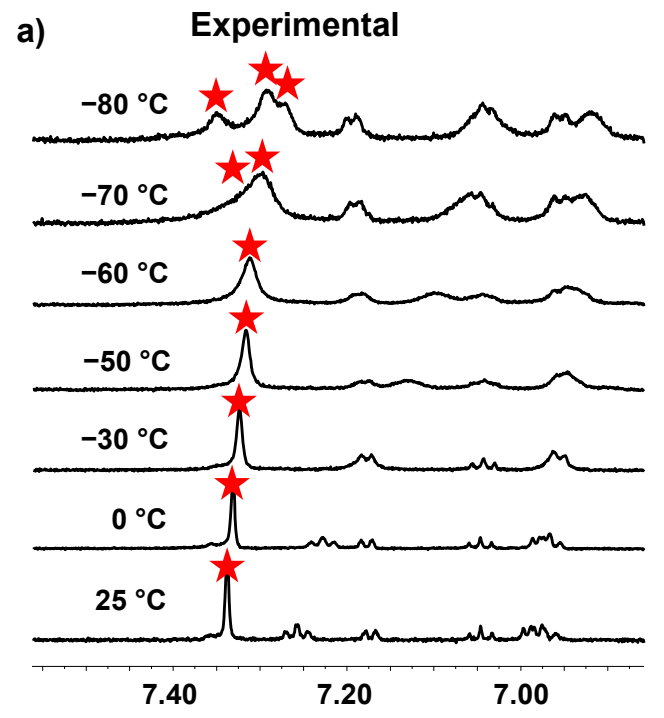

b) Simulated
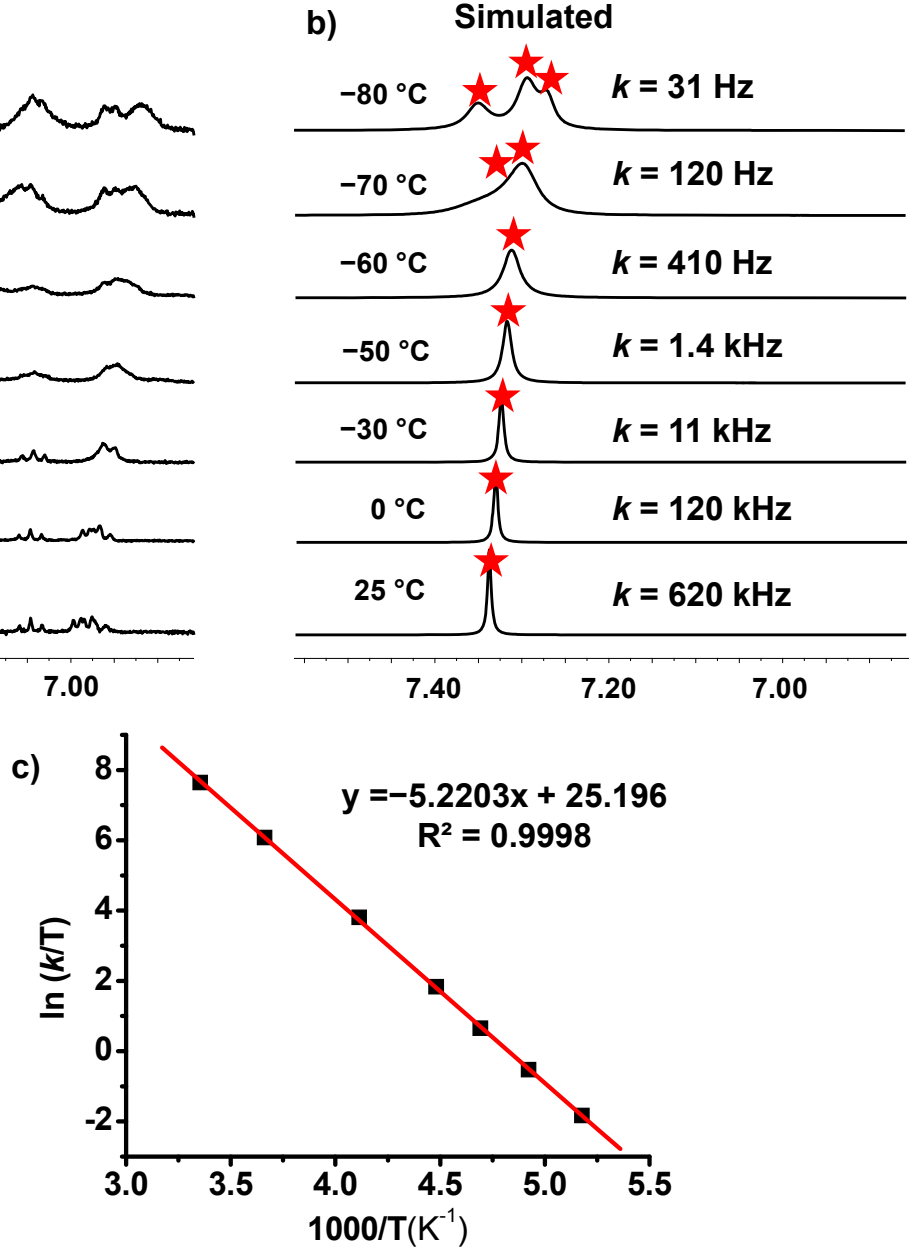

Figure S45. Partial ${ }^{1} \mathrm{H}$ VT-NMR spectra $\left(\mathrm{CD}_{2} \mathrm{Cl}_{2}, 600 \mathrm{MHz}\right)$ of $[\mathrm{Cu}(\mathbf{S})(\mathbf{R 1})]^{+}$at different temperatures showing (a) experimental, (b) theoretical splitting of $\mathrm{m}-\mathrm{H}$ with corresponding rate constants. (c) Eyring plot for the rotational dynamics.

a)

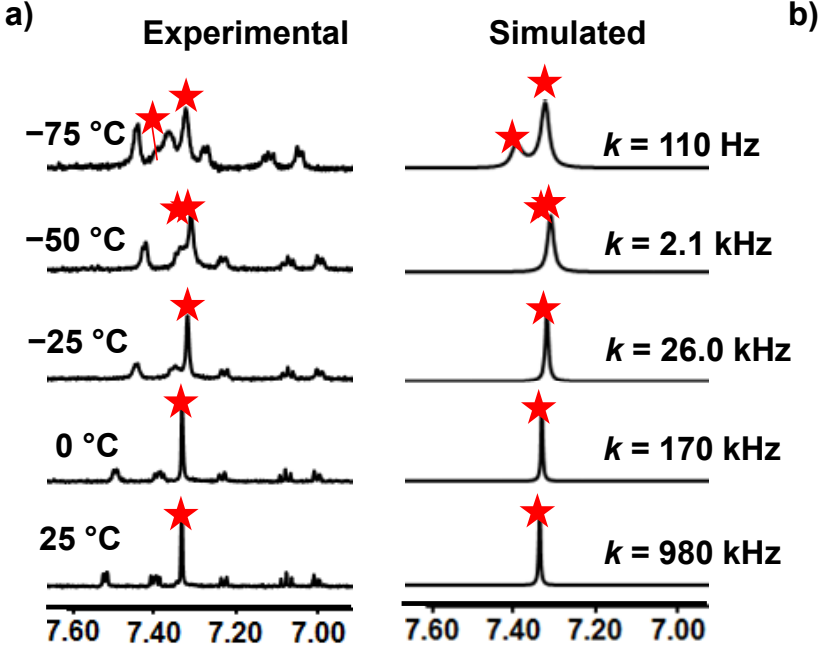

b)

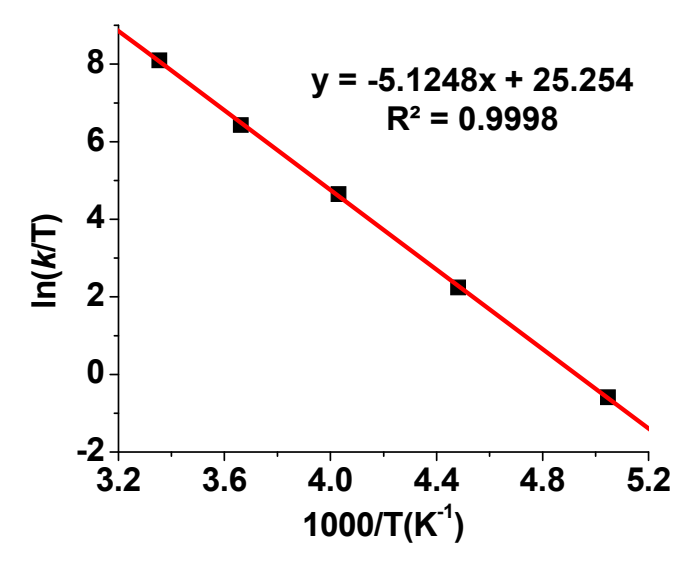

Figure S46. Partial ${ }^{1} \mathrm{H}$ VT-NMR $\left(\mathrm{CD}_{3} \mathrm{CN}: \mathrm{CD}_{2} \mathrm{Cl}_{2}(1: 5), 600 \mathrm{MHz}\right)$ of $[\mathrm{Zn}(\mathbf{S})(\mathbf{R 1})]^{2+}$ showing (a) experimental and theoretical splitting of $\mathrm{m}-\mathrm{H}$ with corresponding rate constants. (b) Eyring plot for rotational dynamics in $[\mathrm{Zn}(\mathbf{S})(\mathbf{R} 1)]^{2+}$. 
a)
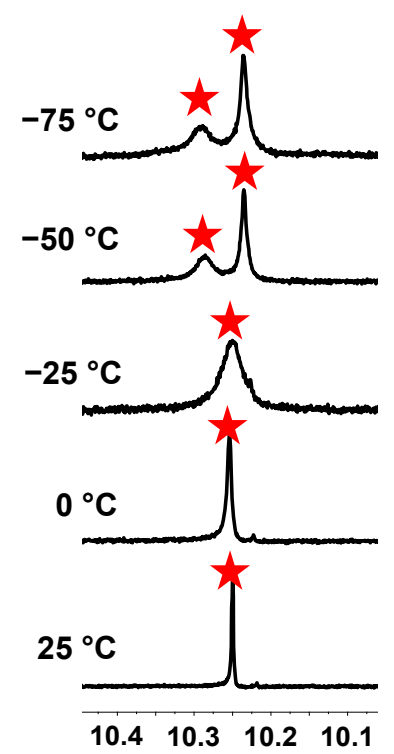

Simulated

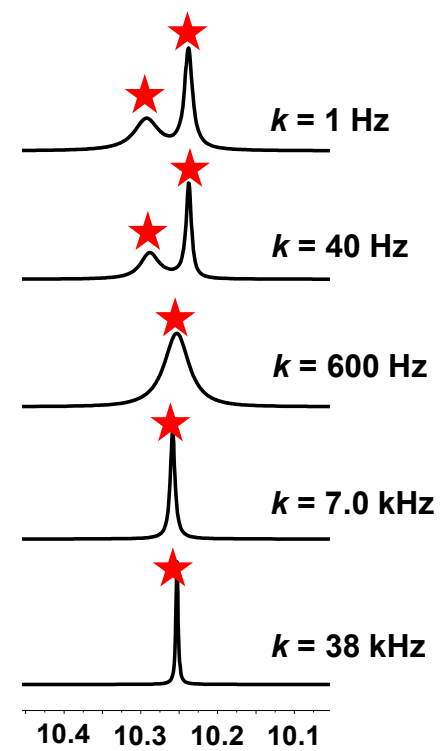

b)

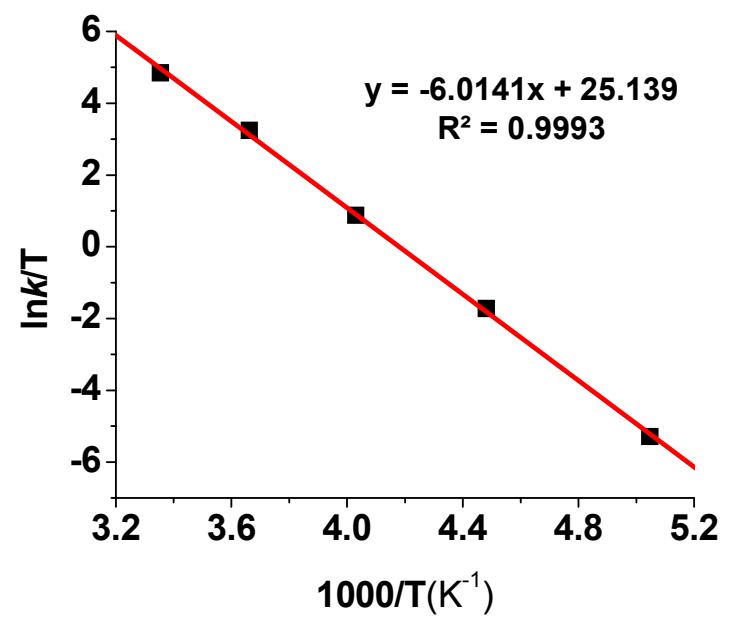

Figure S47. Partial ${ }^{1} \mathrm{H}$ VT-NMR $\left(\mathrm{CD}_{2} \mathrm{Cl}_{2}, 600 \mathrm{MHz}\right)$ of $[\mathrm{Cu}(\mathbf{S})(\mathbf{R} 2)]^{+}$showing (a) experimental and theoretical splitting of r-H with corresponding rate constants. (b) Eyring plot for rotational dynamics in $[\mathrm{Cu}(\mathbf{S})(\mathbf{R 2})]^{+}$.

a)

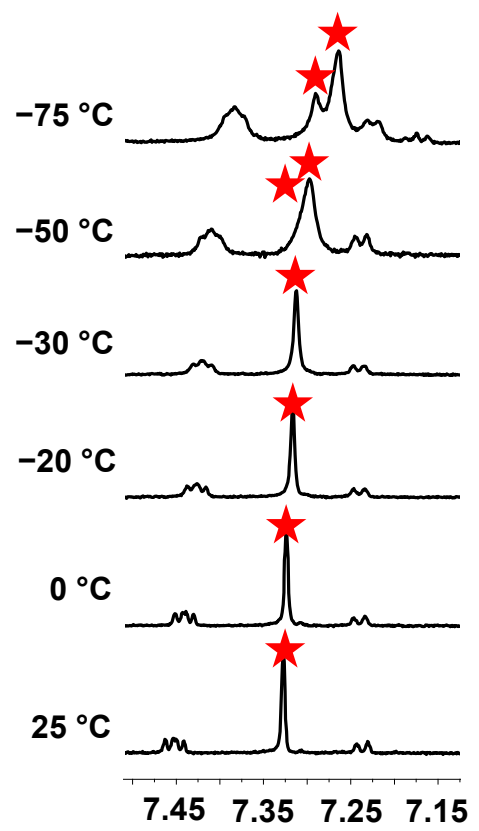

Simulated

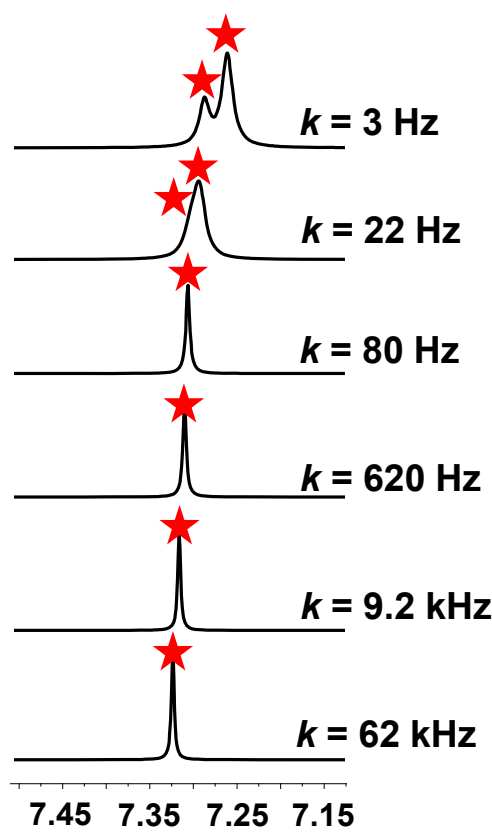

b)

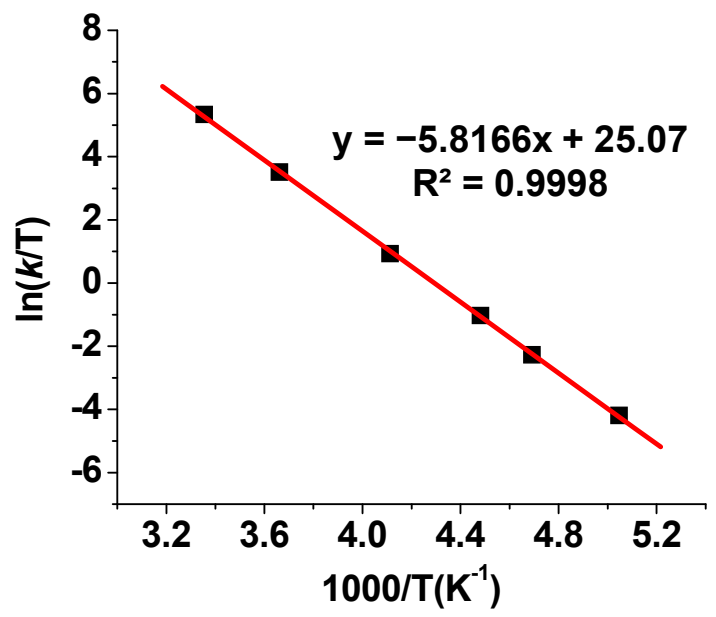

Figure S48. Partial ${ }^{1} \mathrm{H}$ VT-NMR $\left(\mathrm{CD}_{3} \mathrm{CN}: \mathrm{CD}_{2} \mathrm{Cl}_{2}(1: 5), 600 \mathrm{MHz}\right)$ of $[\mathrm{Zn}(\mathbf{S})(\mathbf{R} 2)]^{2+}$ showing (a) experimental and theoretical splitting of $\mathrm{m}-\mathrm{H}$ with corresponding rate constants. (b) Eyring plot for rotational dynamics in $[\mathrm{Zn}(\mathbf{S})(\mathbf{R} 2)]^{2+}$. 


\section{Catalytic experiments}

\section{General procedure}

Solid reactants were transferred to the NMR tube and dissolved in $\mathrm{CD}_{2} \mathrm{Cl}_{2}: \mathrm{CD}_{3} \mathrm{CN}(5: 1)$. The mixture was heated at $50{ }^{\circ} \mathrm{C}$ for $2 \mathrm{~h}$ and the yield of the click product (singlet at $\delta 5.58 \mathrm{ppm}$ ) and 1,4-addition product (multiplet at $\delta 4.86 \mathrm{ppm}$ ) was determined using 1,3,5-trimethoxybenzene (14) as an internal standard (singlet at $\delta 6.04 \mathrm{ppm})$.

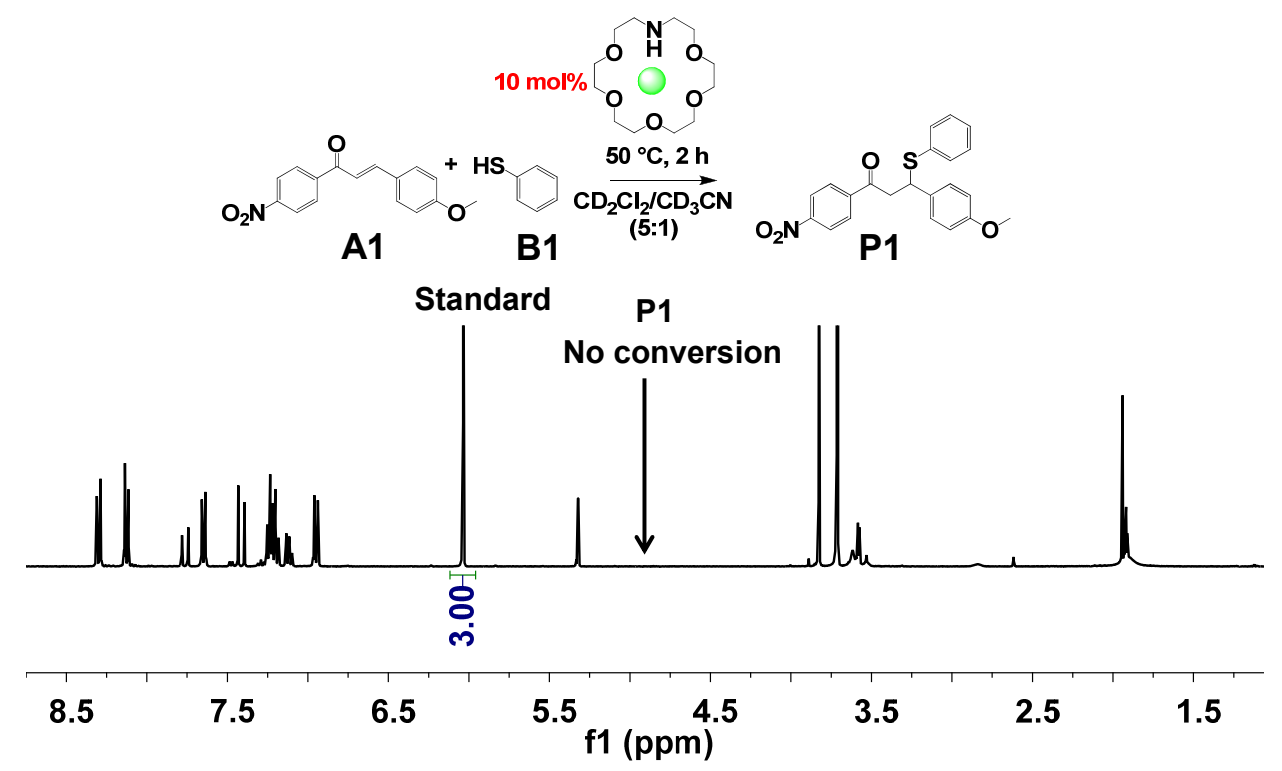

Figure S49. ${ }^{1} \mathrm{H}$ NMR (400 MHz, $\left.\mathrm{CD}_{2} \mathrm{Cl}_{2}: \mathrm{CD}_{3} \mathrm{CN}=5: 1,298 \mathrm{~K}\right)$ spectrum obtained after heating the reaction mixture of A1, B1, $7(\approx 1.92 \mathrm{mM}),\left[\mathrm{Cu}\left(\mathrm{CH}_{3} \mathrm{CN}\right)_{4}\right] \mathrm{PF}_{6}$ and standard $\mathbf{1 4}$ in 10:10:1:1:10 ratio at $50{ }^{\circ} \mathrm{C}$ for $2 \mathrm{~h}$. No product $\mathbf{P 1}$ was observed in ${ }^{1} \mathrm{H}$ NMR.

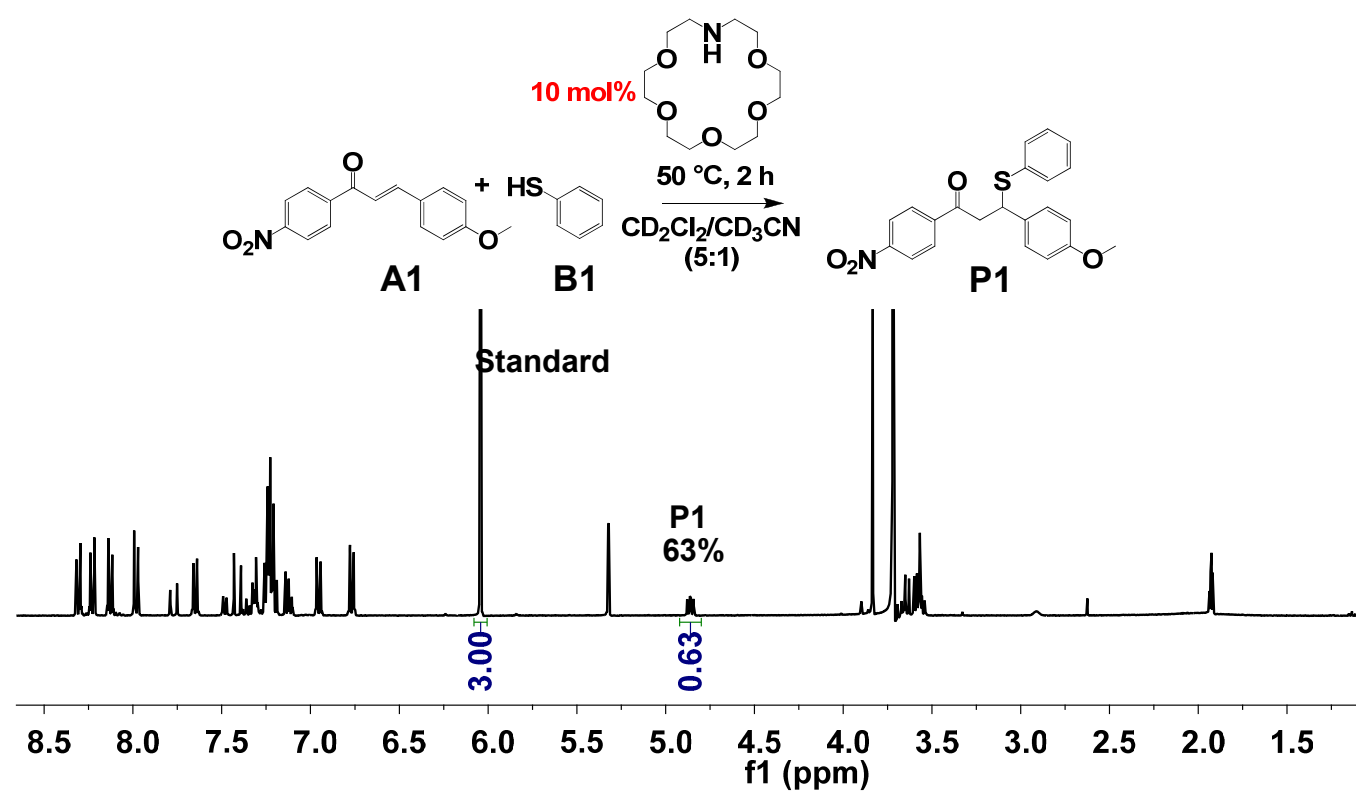

Figure S50. ${ }^{1} \mathrm{H}$ NMR (400 MHz, $\mathrm{CD}_{2} \mathrm{Cl}_{2}: \mathrm{CD}_{3} \mathrm{CN}=5: 1,298 \mathrm{~K}$ ) spectrum obtained after heating the reaction mixture of A1, B1, $7(\approx 1.92 \mathrm{mM})$, and standard $\mathbf{1 4}$ in 10:10:1:10 ratio at $50{ }^{\circ} \mathrm{C}$ for $2 \mathrm{~h}$. The integration demonstrated that $\mathbf{P 1}$ was formed in $63 \%$ yield. 
<smiles>O=C(CCC1CCC([O-])CC1)C1CCC([N+](=O)[O-])CC1</smiles>

A1

\section{B1}

$10 \mathrm{~mol} \%[(4)(7)]$

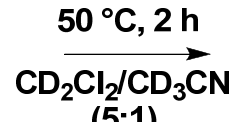

(5:1)

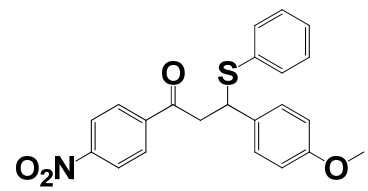

P1

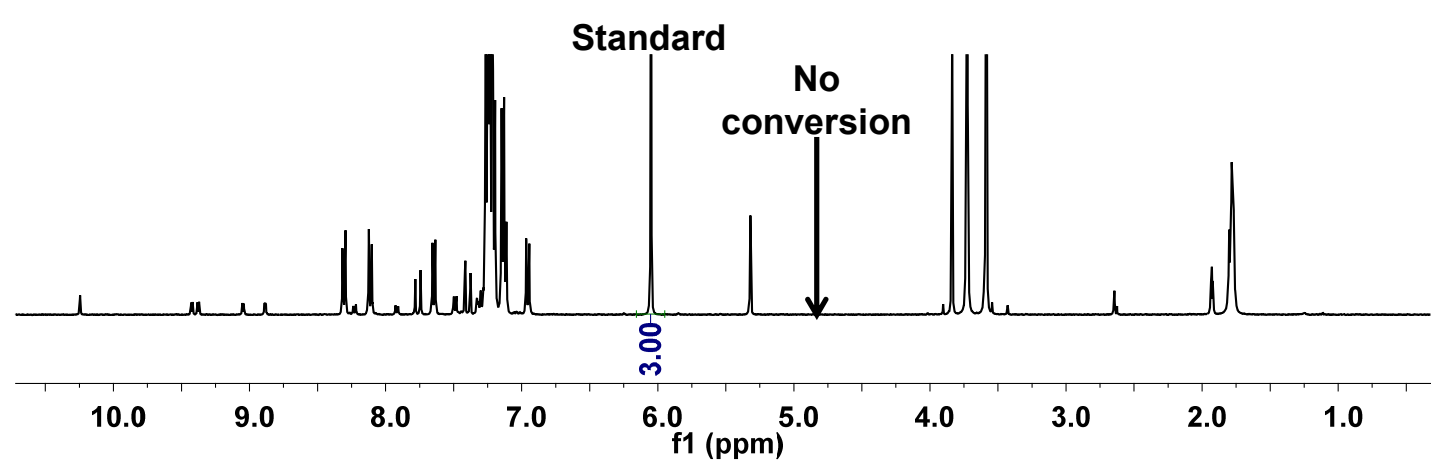

Figure S51. ${ }^{1} \mathrm{H}$ NMR (400 MHz, $\mathrm{CD}_{2} \mathrm{Cl}_{2}: \mathrm{CD}_{3} \mathrm{CN}=5: 1,298 \mathrm{~K}$ ) spectrum obtained after heating the reaction mixture of A1, B1, 4, $7(\approx 1.92 \mathrm{mM})$, and standard $\mathbf{1 4}$ in 10:10:1:1:10 ratio at $50{ }^{\circ} \mathrm{C}$ for $2 \mathrm{~h}$. No product was observed in ${ }^{1} \mathrm{H}$ NMR.

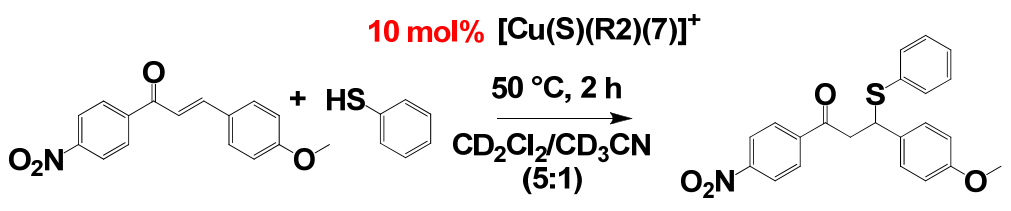

A1

B1

P1

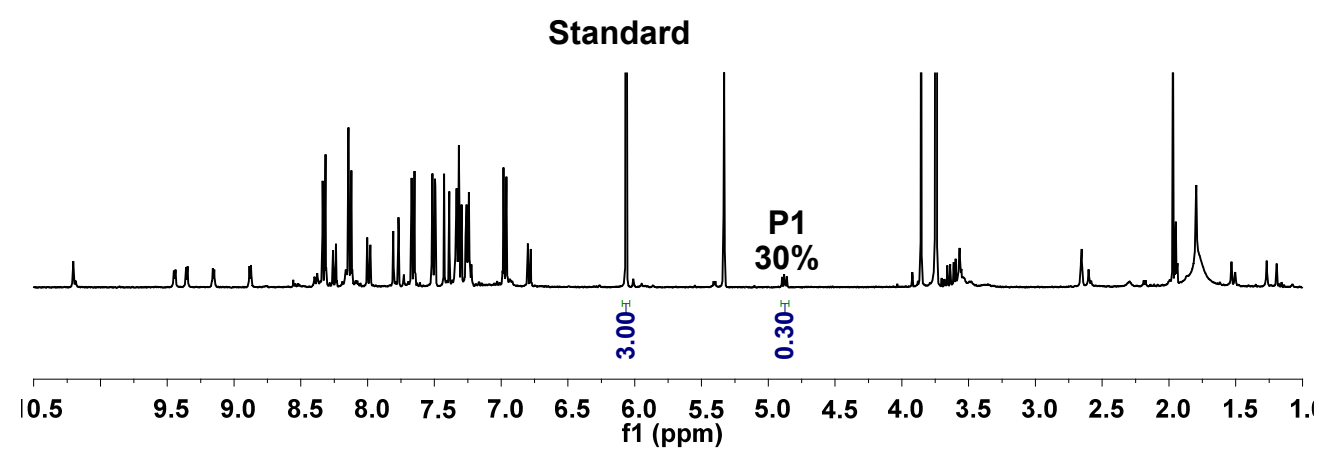

Figure S52. ${ }^{1} \mathrm{H}$ NMR (400 MHz, $\mathrm{CD}_{2} \mathrm{Cl}_{2}: \mathrm{CD}_{3} \mathrm{CN}=5: 1,298 \mathrm{~K}$ ) spectrum obtained after heating the reaction mixture of A1, B1, $7 \approx 1.92 \mathrm{mM}),[\mathrm{Cu}(\mathbf{S})(\mathbf{R 2})]^{+}$and standard 14 in $10: 10: 1: 1: 10$ ratio at $50{ }^{\circ} \mathrm{C}$ for $2 \mathrm{~h}$. The integration demonstrated that $\mathbf{P 1}$ was formed in $30 \%$ yield. 


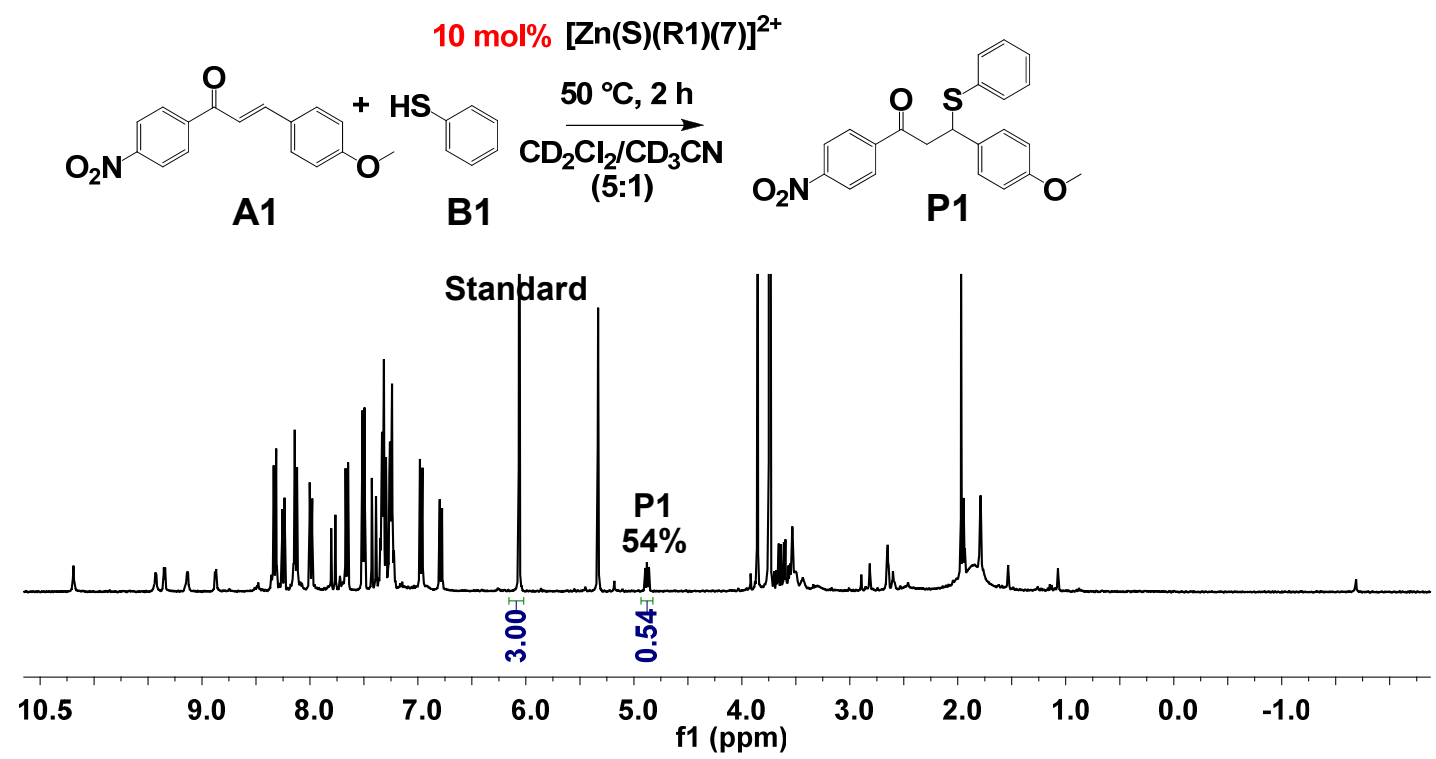

Figure S53. ${ }^{1} \mathrm{H}$ NMR ( $\left.400 \mathrm{MHz}, \mathrm{CD}_{2} \mathrm{Cl}_{2}: \mathrm{CD}_{3} \mathrm{CN}=5: 1,298 \mathrm{~K}\right)$ spectrum obtained after heating the reaction mixture of A1, B1, $7 \approx 1.92 \mathrm{mM}),[\operatorname{Zn}(\mathbf{S})(\mathbf{R} 1)]^{2+}$ and standard 14 in $10: 10: 1: 1: 10$ ratio at $50{ }^{\circ} \mathrm{C}$ for $2 \mathrm{~h}$. The integration demonstrated that $\mathbf{P 1}$ was formed in 54\% yield.
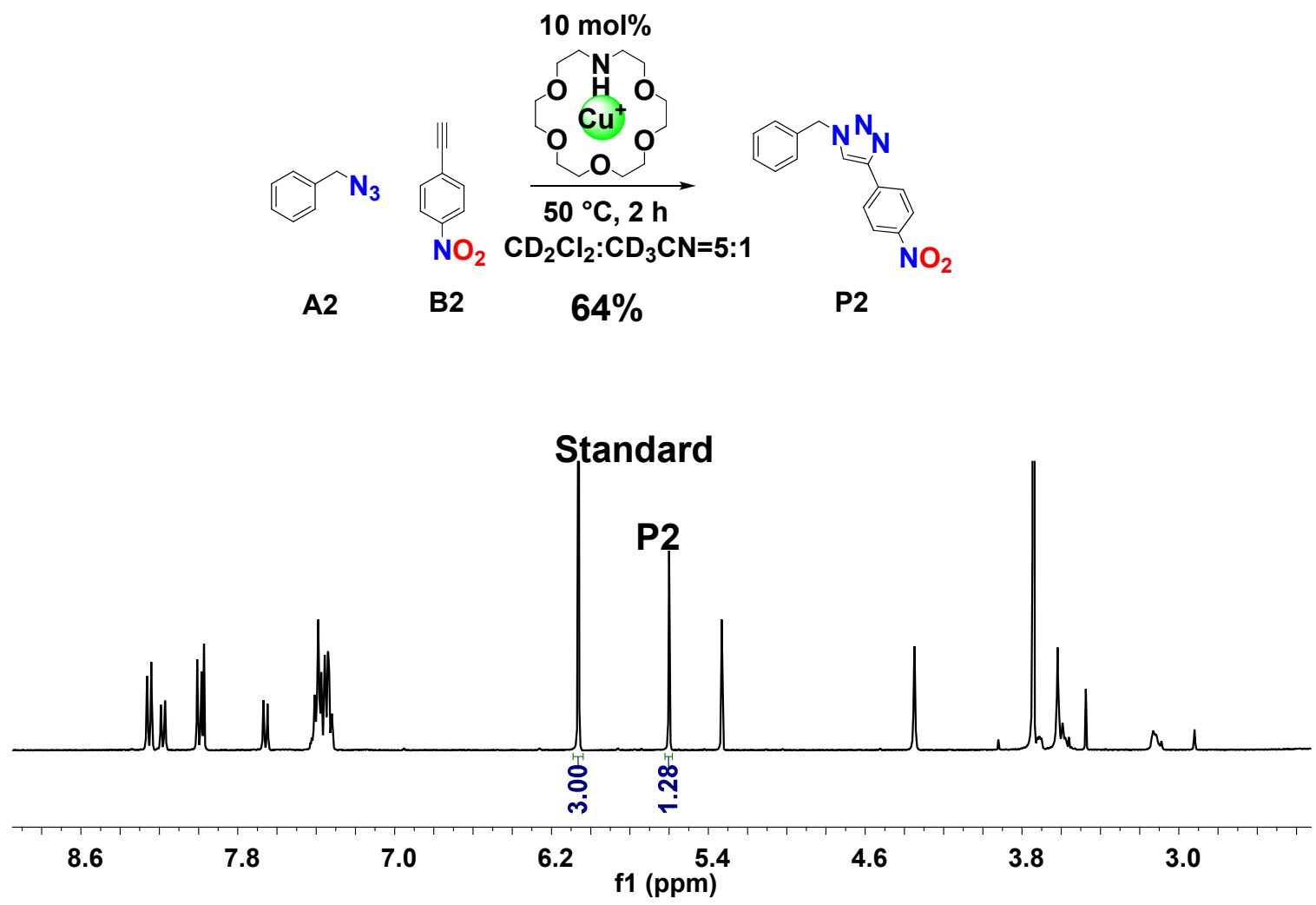

Figure S54. ${ }^{1} \mathrm{H}$ NMR $\left(400 \mathrm{MHz}, \mathrm{CD}_{2} \mathrm{Cl}_{2}: \mathrm{CD}_{3} \mathrm{CN}=5: 1,298 \mathrm{~K}\right.$ ) spectrum obtained after heating the reaction mixture of A2, B2, $7(\approx 1.92 \mathrm{mM}),\left[\mathrm{Cu}\left(\mathrm{CH}_{3} \mathrm{CN}\right)_{4}\right] \mathrm{PF}_{6}$ and standard $\mathbf{1 4}$ in 10:10:1:1:10 ratio at $50{ }^{\circ} \mathrm{C}$ for $2 \mathrm{~h}$. The integration demonstrated that $\mathbf{P 2}$ was formed in $64 \%$ yield. 


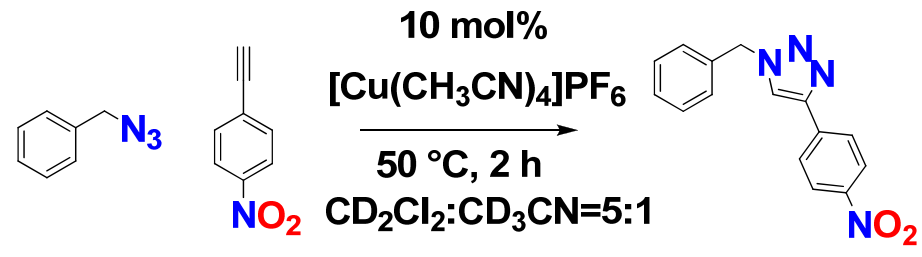

A2

B2

$3 \%$

P2

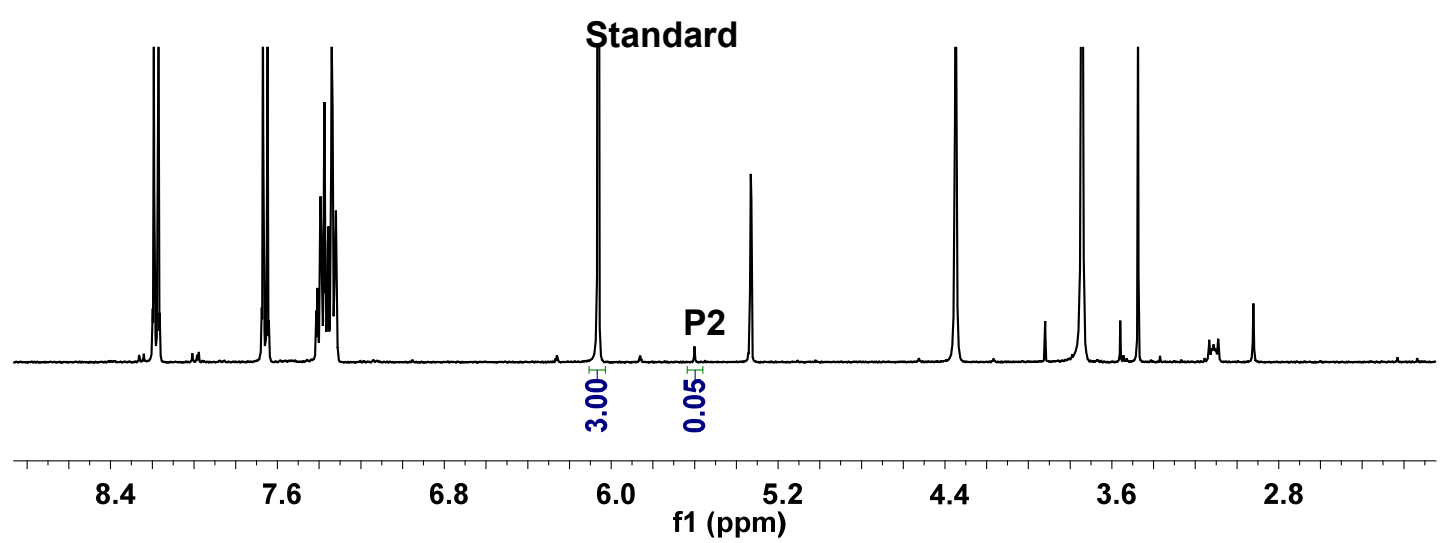

Figure S55. ${ }^{1} \mathrm{H} \mathrm{NMR}\left(400 \mathrm{MHz}, \mathrm{CD}_{2} \mathrm{Cl}_{2}: \mathrm{CD}_{3} \mathrm{CN}=5: 1,298 \mathrm{~K}\right)$ spectrum obtained after heating the reaction mixture of A2, B2, $\left[\mathrm{Cu}\left(\mathrm{CH}_{3} \mathrm{CN}\right)_{4}\right] \mathrm{PF}_{6}(\approx 1.92 \mathrm{mM})$ and standard 14 in 10:10:1:10 ratio at $50{ }^{\circ} \mathrm{C}$ for $2 \mathrm{~h}$. The integration demonstrated that only $\mathbf{P 2}$ was formed in $3 \%$ yield.
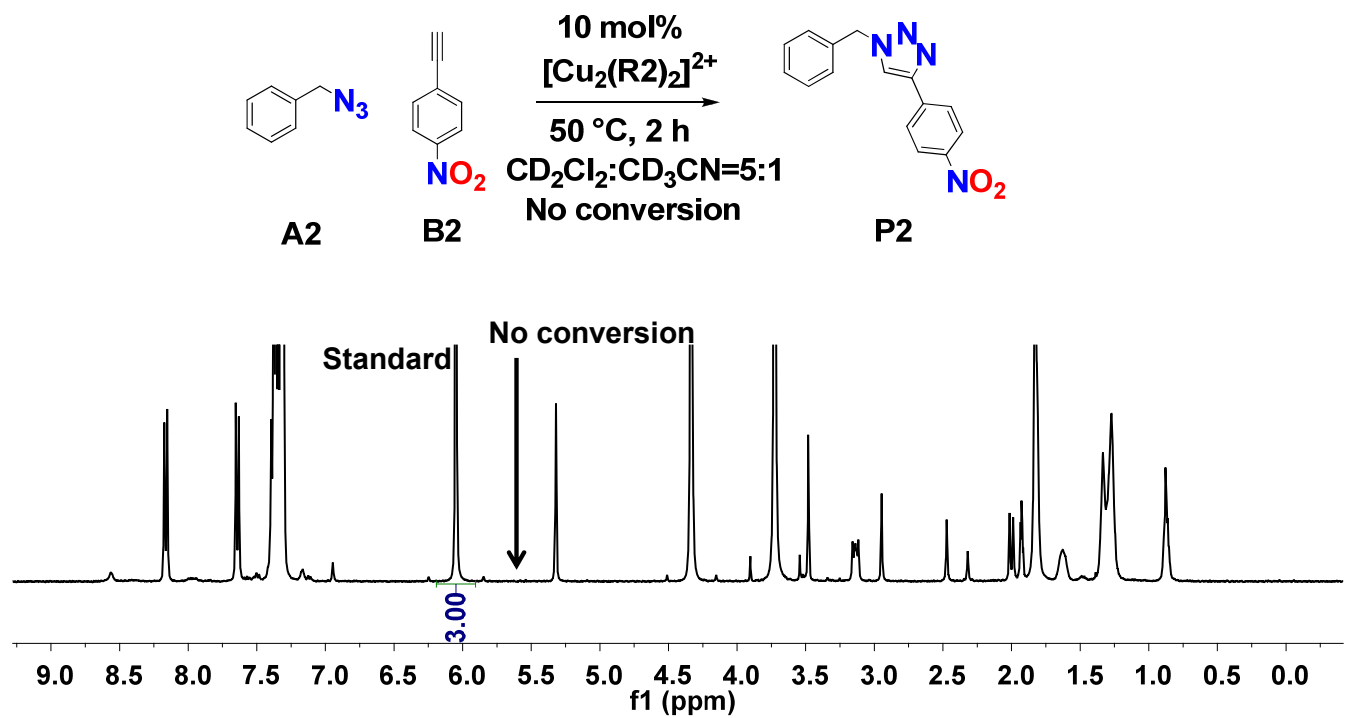

Figure S56. ${ }^{1} \mathrm{H}$ NMR (400 MHz, $\left.\mathrm{CD}_{2} \mathrm{Cl}_{2}: \mathrm{CD}_{3} \mathrm{CN}=5: 1,298 \mathrm{~K}\right)$ spectrum obtained after heating the reaction mixture of A2, B2, $\left[\mathrm{Cu}_{2}(\mathbf{R 2})_{2}\right]^{2+}(\approx 1.92 \mathrm{mM})$ and standard 14 in 10:10:1:10 ratio at $50{ }^{\circ} \mathrm{C}$ for $2 \mathrm{~h}$. No conversion was detected in ${ }^{1} \mathrm{H}$ NMR. 


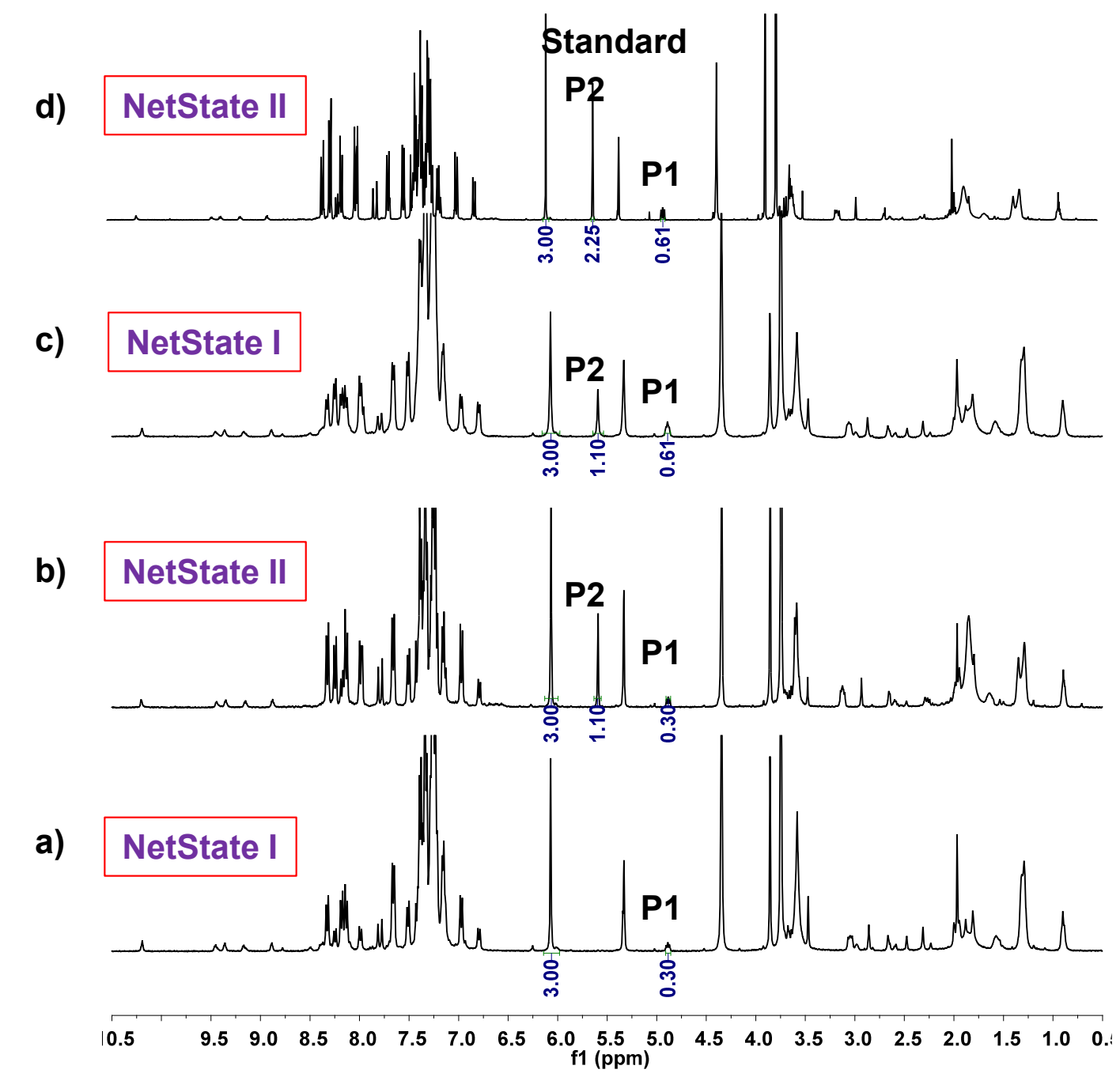

Figure S57. ${ }^{1} \mathrm{H}$ NMR ( $\left.400 \mathrm{MHz}, \mathrm{CD}_{2} \mathrm{Cl}_{2}: \mathrm{CD}_{3} \mathrm{CN}=5: 1,298 \mathrm{~K}\right)$ spectrum obtained after (a) heating the reaction mixture of A1, B1, A2, B2, $7(\approx 1.92 \mathrm{mM}),\left[\mathrm{Cu}_{2}(\mathbf{R} 1)_{2}\right]^{2+},[\mathrm{Cu}(\mathbf{S})(\mathbf{R 2})]^{+}$and standard 14 in 20:20:20:20:2:1:2:20 ratio at $50{ }^{\circ} \mathrm{C}$ for $2 \mathrm{~h}$ in an NMR tube revealed $30 \%$ of 1,4 addition product $\mathbf{P 1}$ was formed. (b) After addition of 1.0 equiv. of $\mathrm{Zn}(\mathrm{OTf})_{2}$ with respect to nanorotor $[\mathrm{Cu}(\mathbf{S})(\mathbf{R 2})]^{+}$and subsequent heating at $50{ }^{\circ} \mathrm{C}$ for $2 \mathrm{~h}$, the click product $\mathbf{P 2}$ was formed (yield $=55 \%$ calculated with respect to internal standard 14). In contrast, there is no increment in the yield of P1 observed (30\%). (c) After adding 2.0 equiv. of hexacyclen with respect to nanorotor and heating at $50{ }^{\circ} \mathrm{C}$ for $2 \mathrm{~h}, 31 \%$ increase in the amount of $\mathbf{P 1}$ was observed (yield $=61 \%$ ), whereas no further conversion of $\mathbf{P 2}$ was observed. (d) Addition of another 2.0 equiv. of $\mathrm{Zn}(\mathrm{OTf})_{2}$ with respect to nanorotor and heating at $50{ }^{\circ} \mathrm{C}$ for $2 \mathrm{~h}$ resulted in an increase of the click product $\mathbf{P 2}$ by 57\% (total yield $=112 \%$ ); no further conversion of $\mathbf{P 1}$ was detected. 


\section{ESI-MS Spectra}

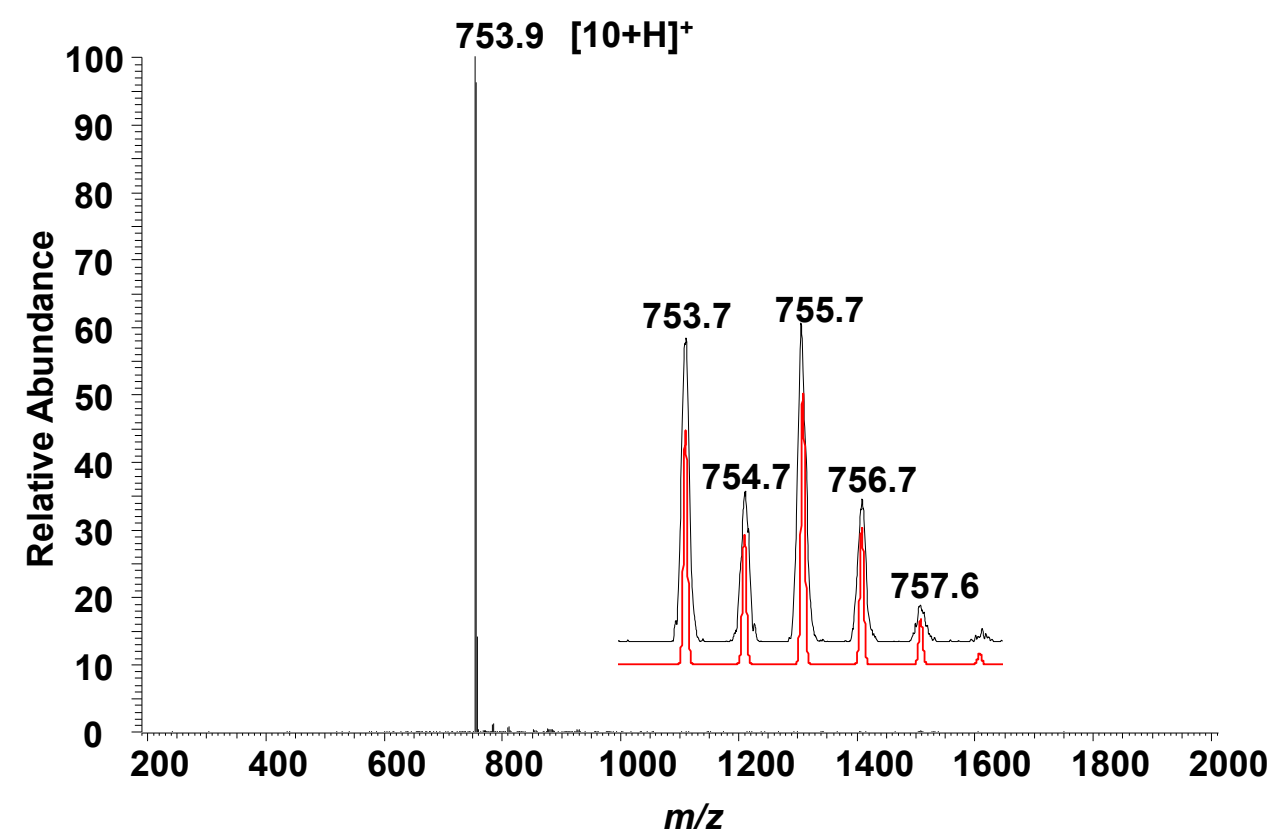

Figure S58. ESI-MS of compound $\mathbf{1 0}$ after protonation.

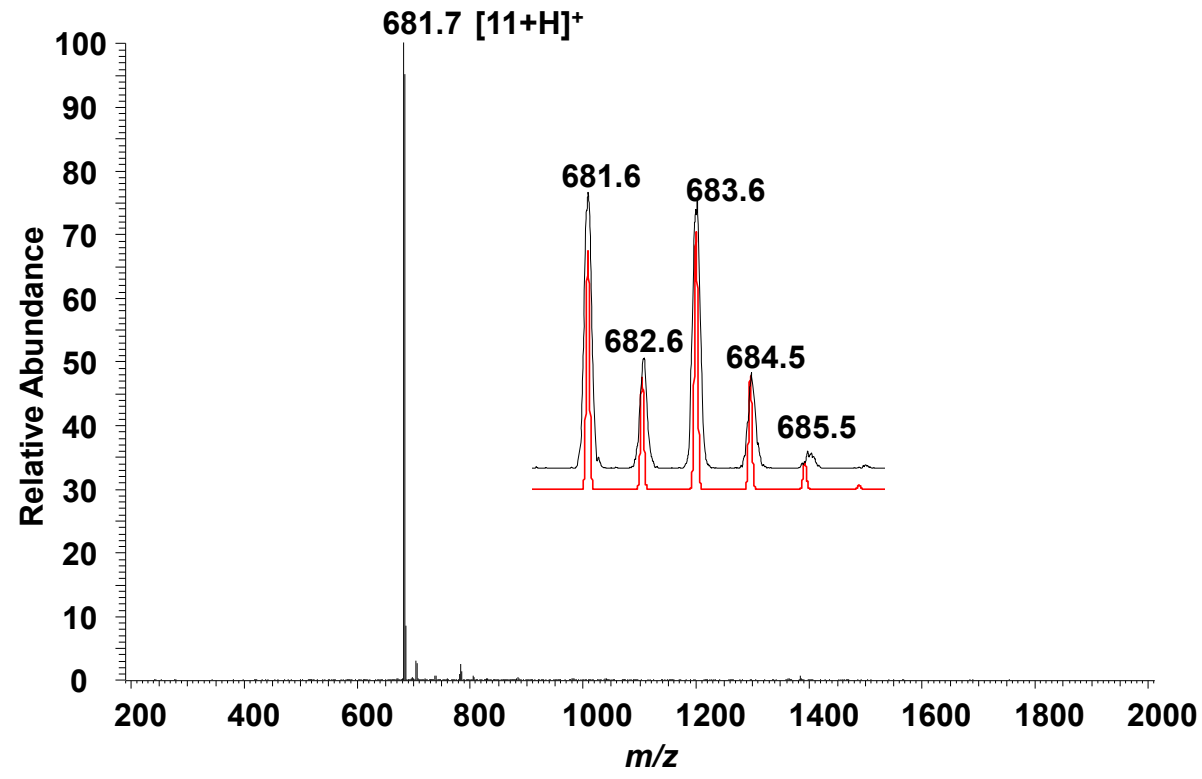

Figure S59. ESI-MS of 11 after protonation. 


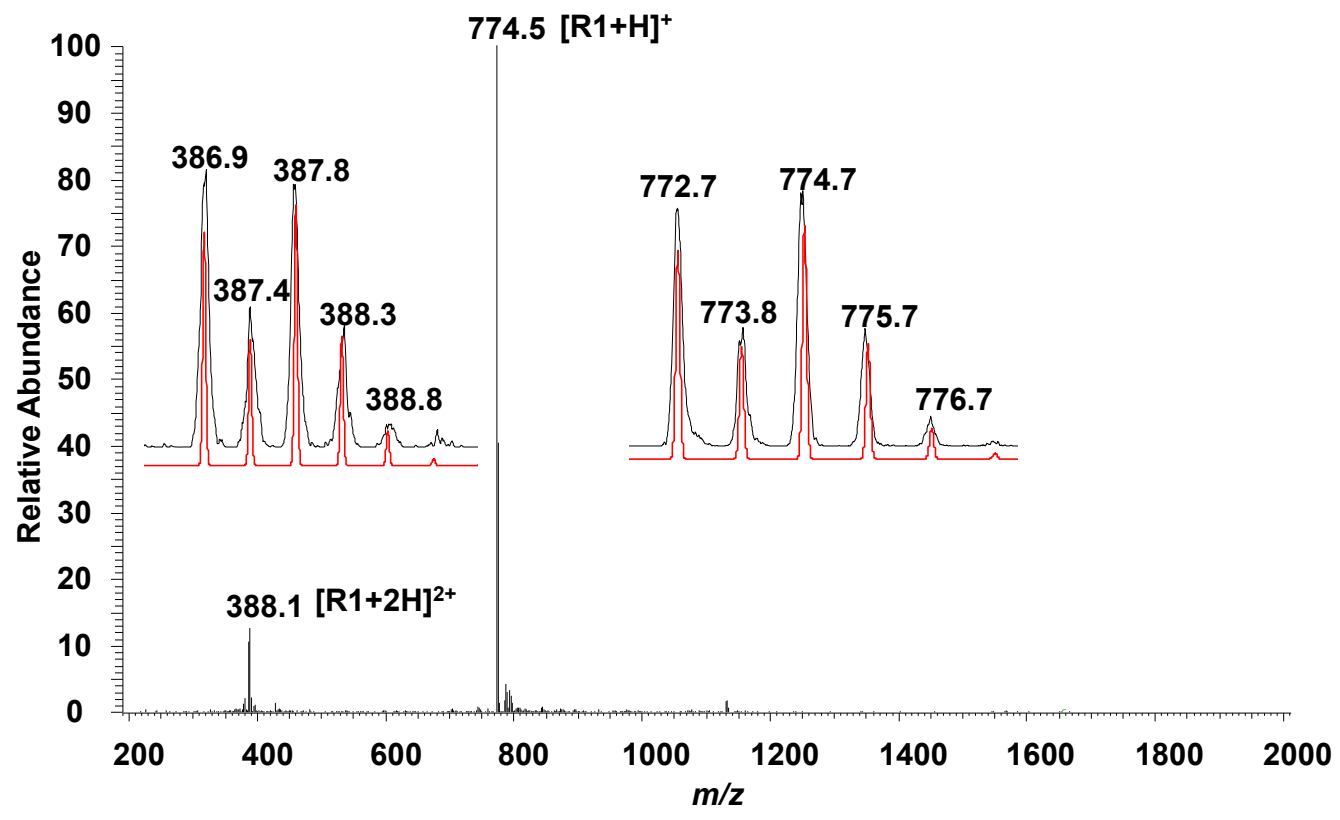

Figure S60. ESI-MS of R1 after protonation.

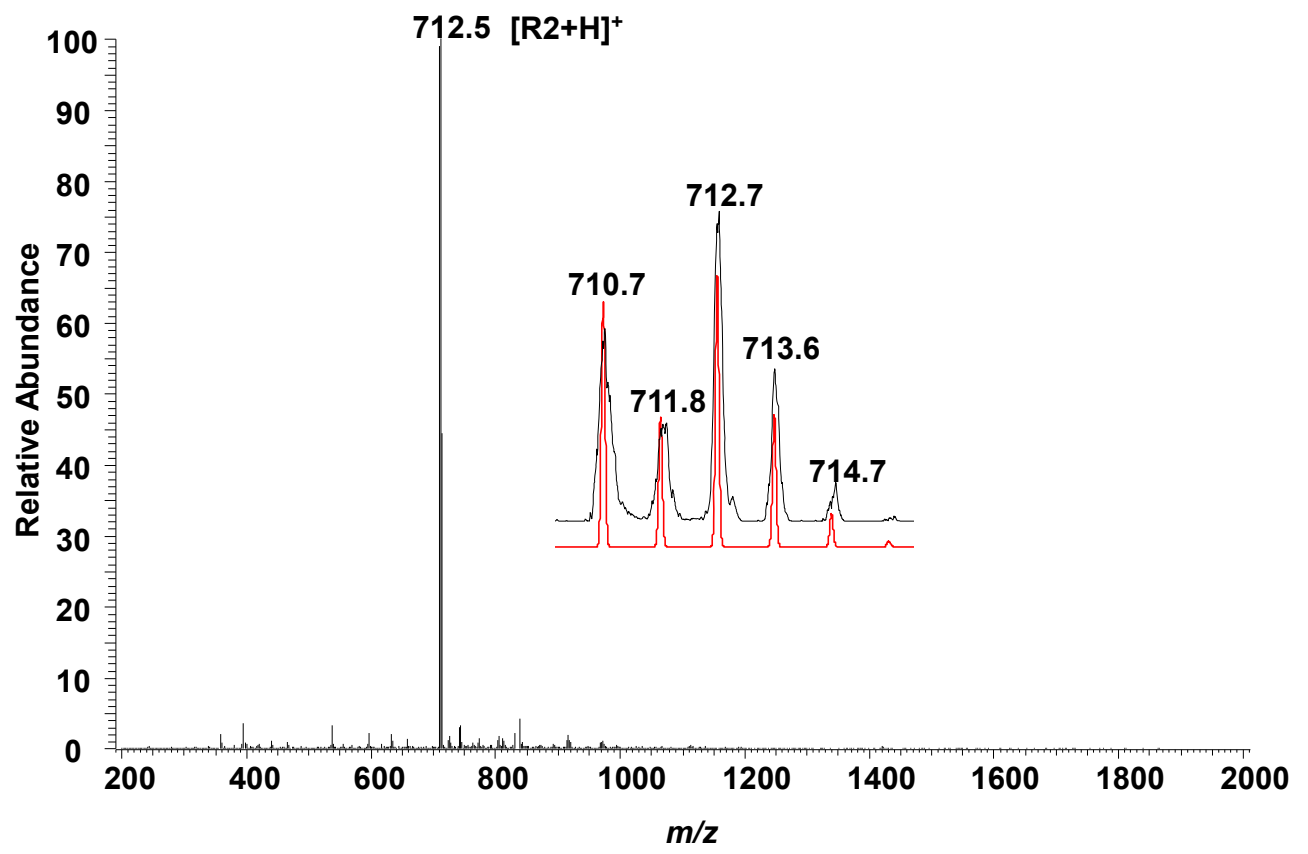

Figure S61. ESI-MS of $\mathbf{R 2}$ after protonation. 


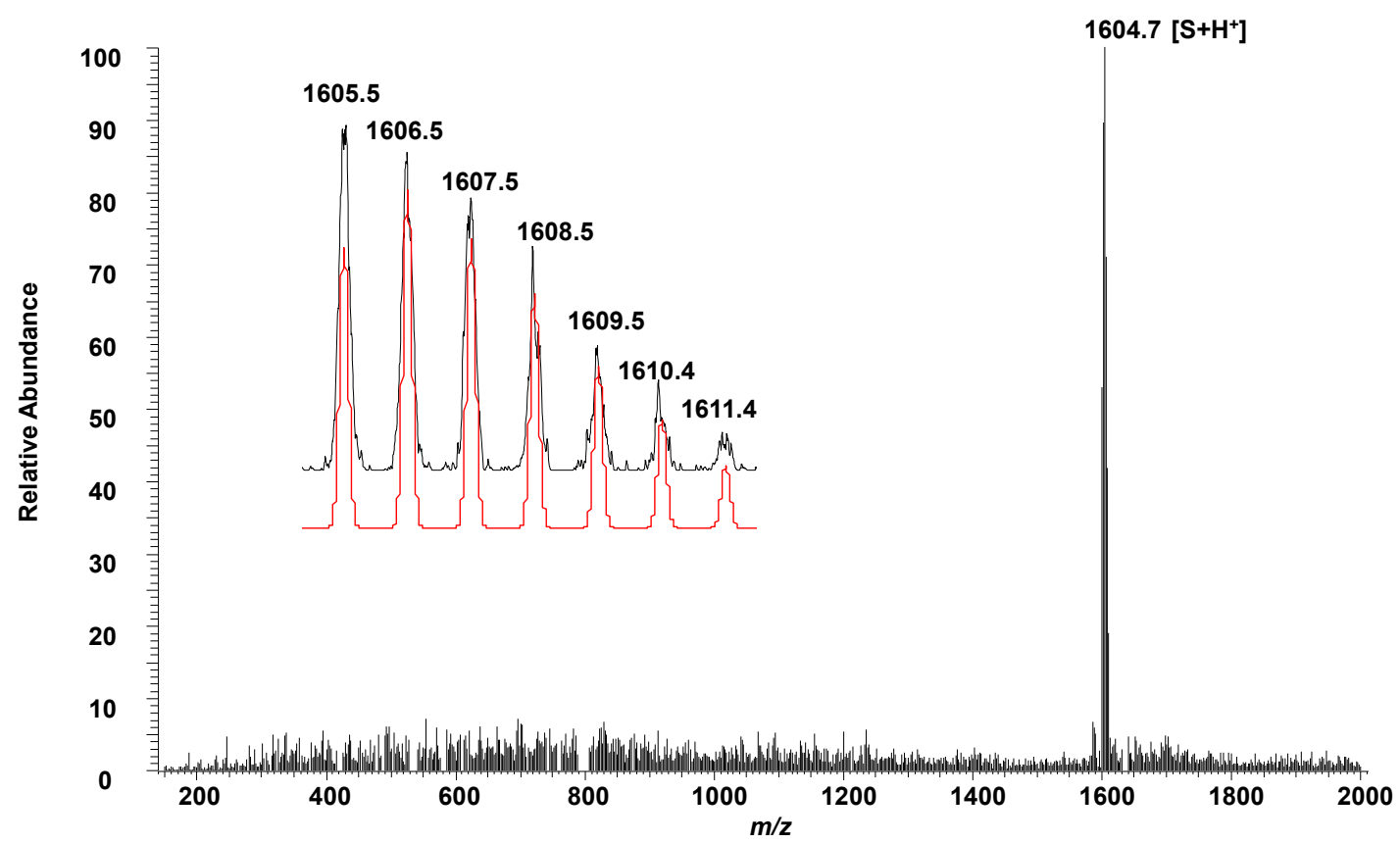

Figure S62. ESI-MS of compound $\mathbf{S}$ after protonation.

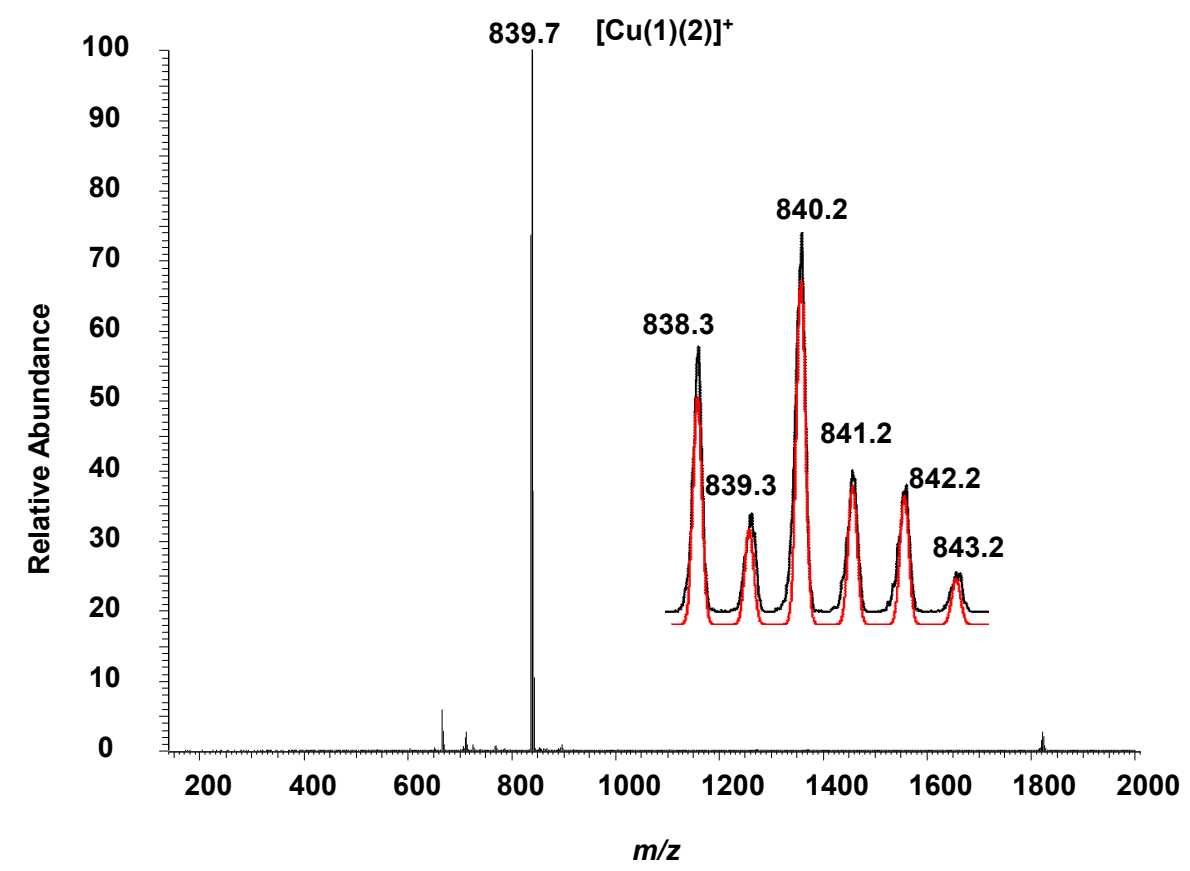

Figure S63. ESI-MS of $[\mathrm{Cu}(1)(2)]^{+}$. 


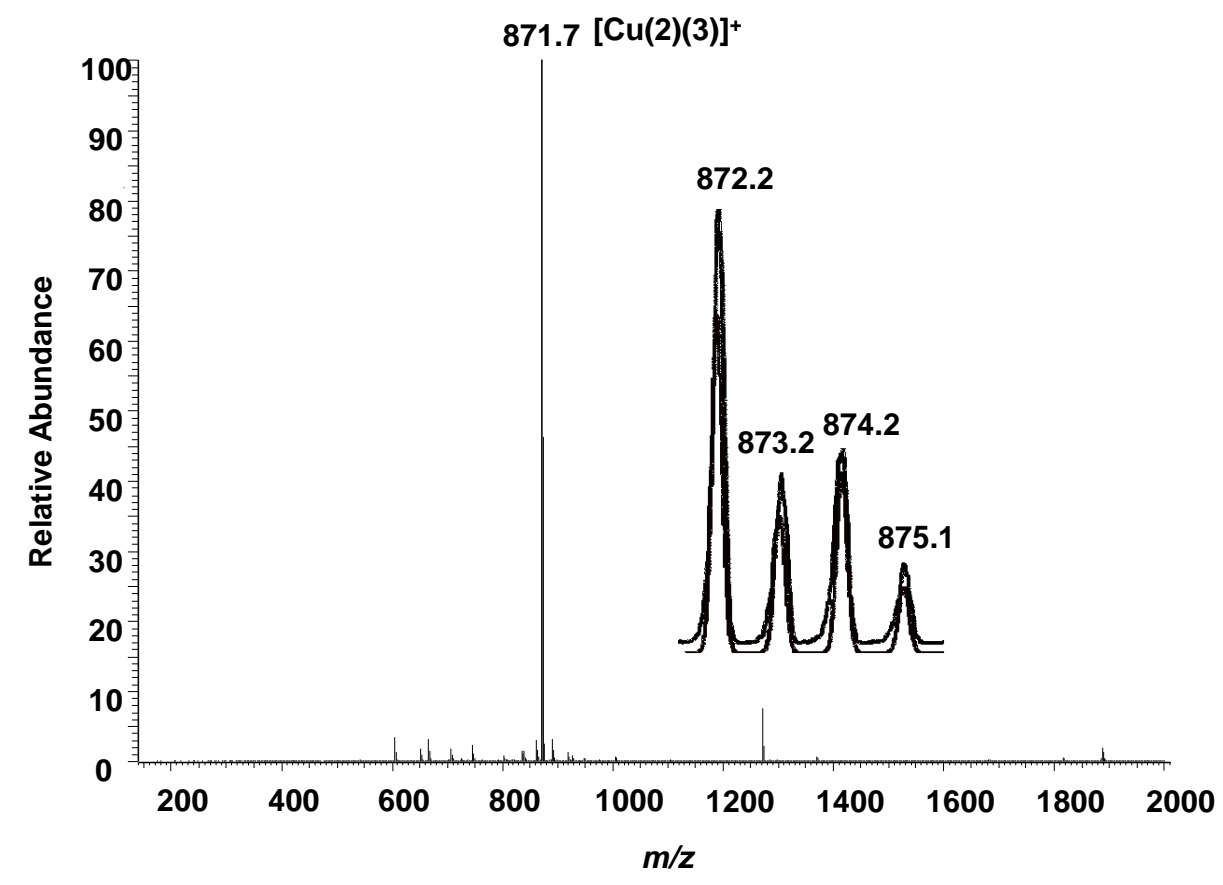

Figure S64. ESI-MS of $[\mathrm{Cu}(2)(3)]^{+}$.

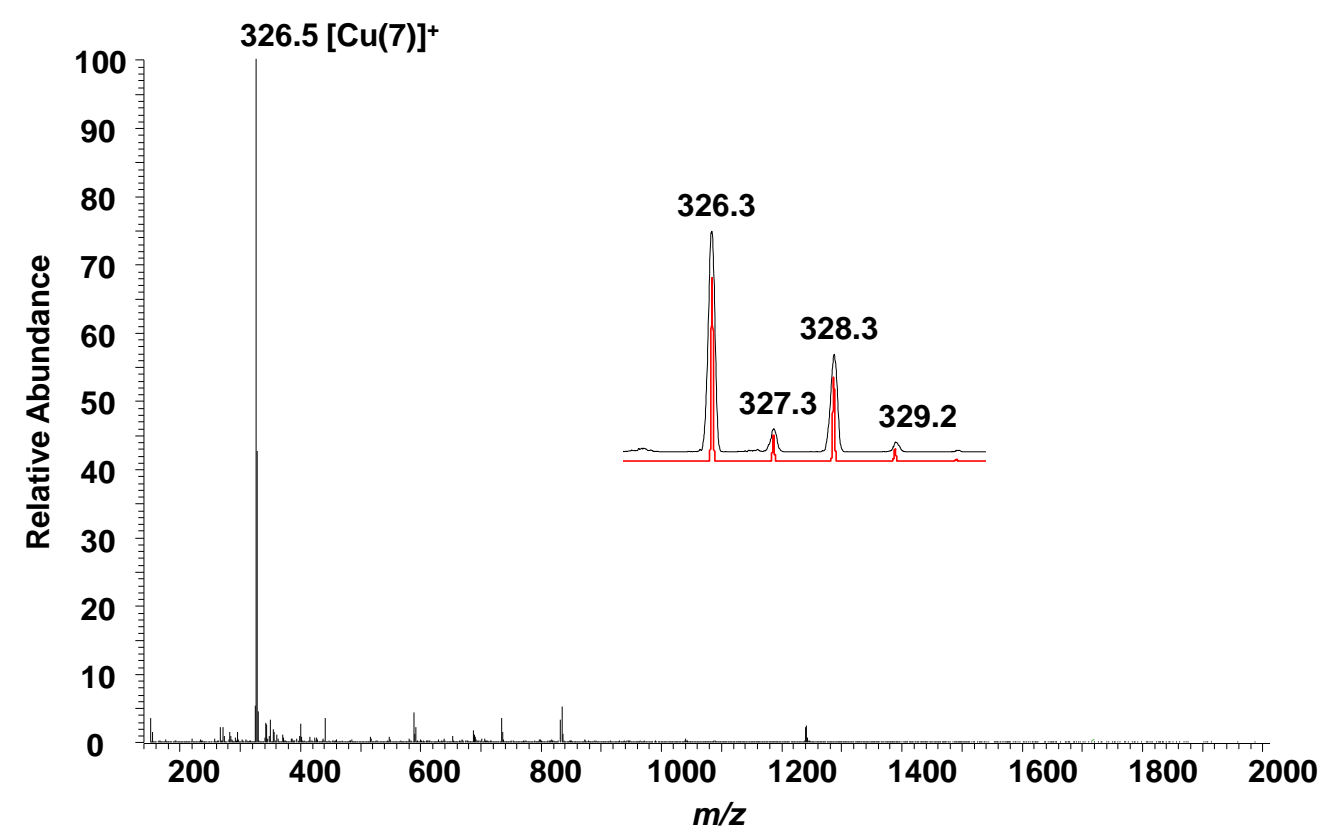

Figure S65. ESI-MS of $[\mathrm{Cu}(7)]^{+}$. 


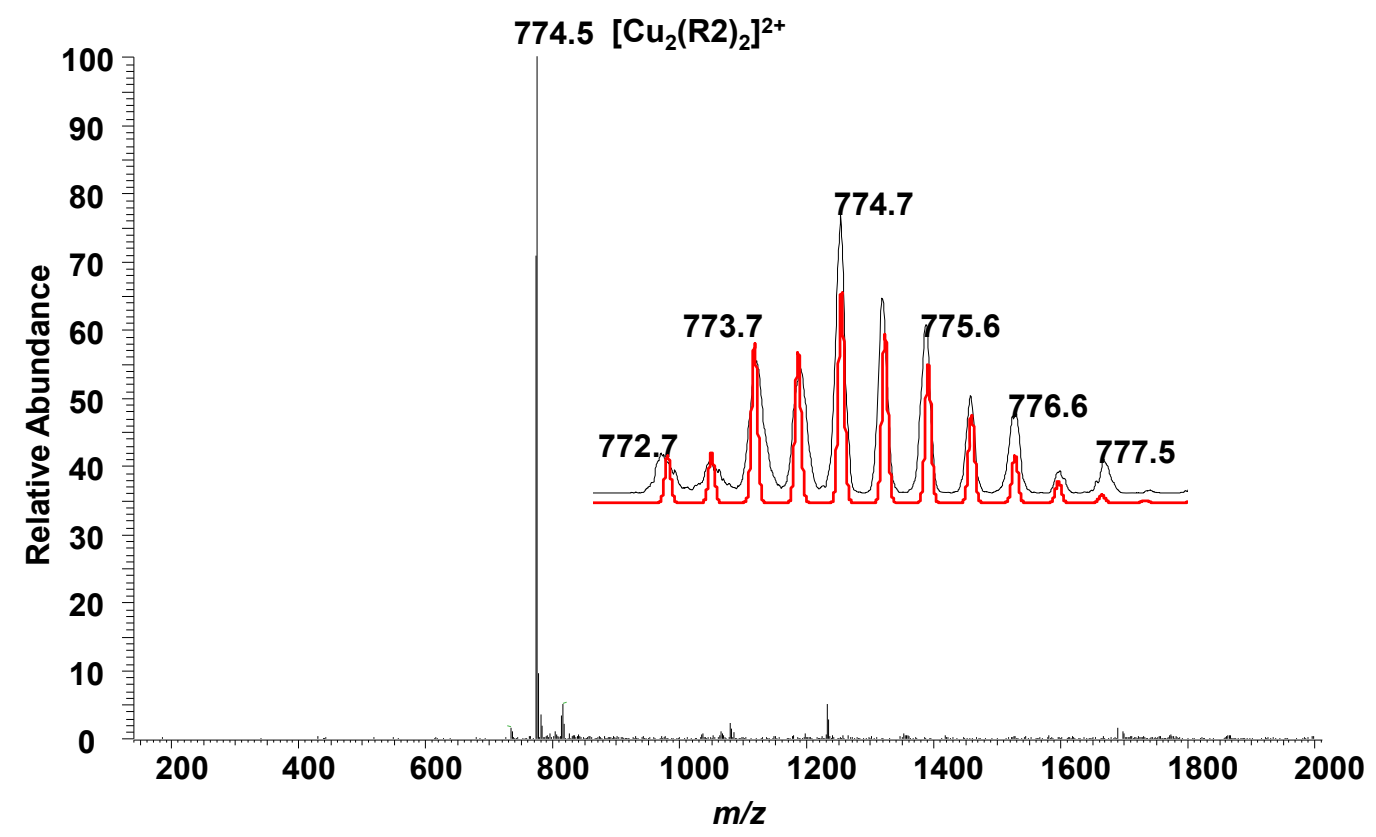

Figure S66. ESI-MS of $\left[\mathrm{Cu}_{2}(\mathbf{R 2})_{2}\right]^{2+}$.

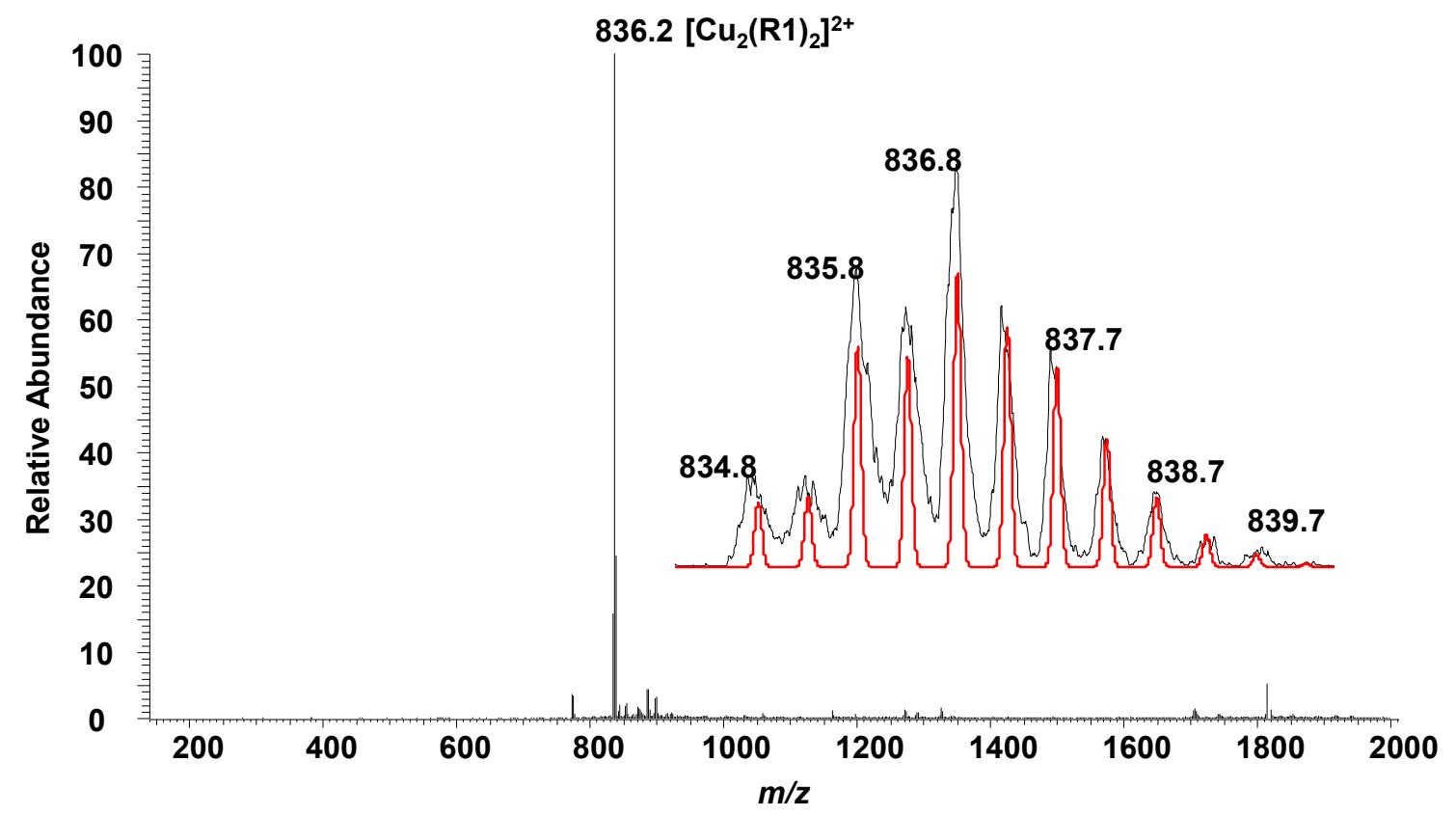

Figure S67. ESI-MS of $\left[\mathrm{Cu}_{2}(\mathbf{R 1})_{2}\right]^{2+}$. 


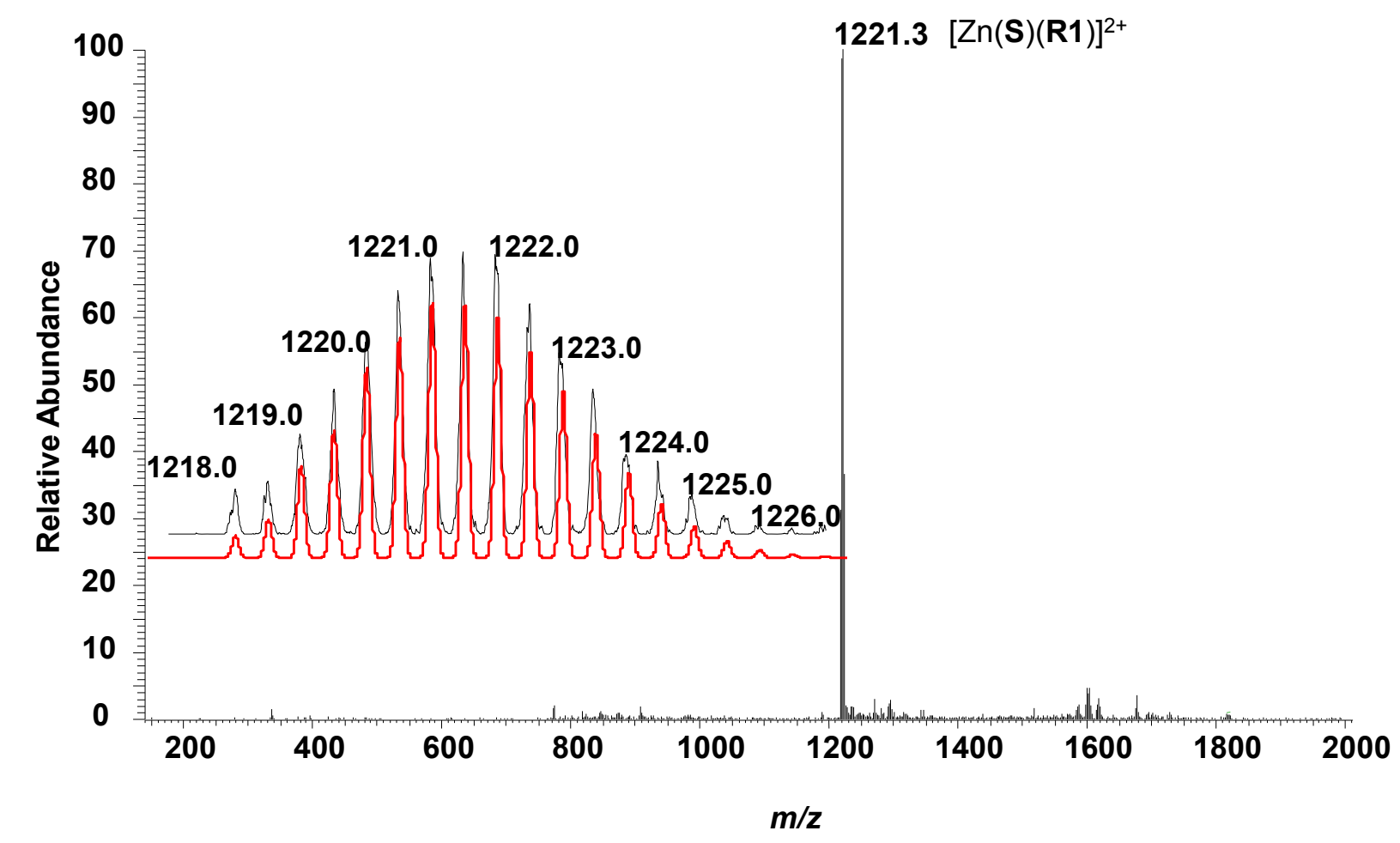

Figure S68. ESI-MS of nanorotor $[\mathrm{Zn}(\mathbf{S})(\mathbf{R 1})]^{2+}$.

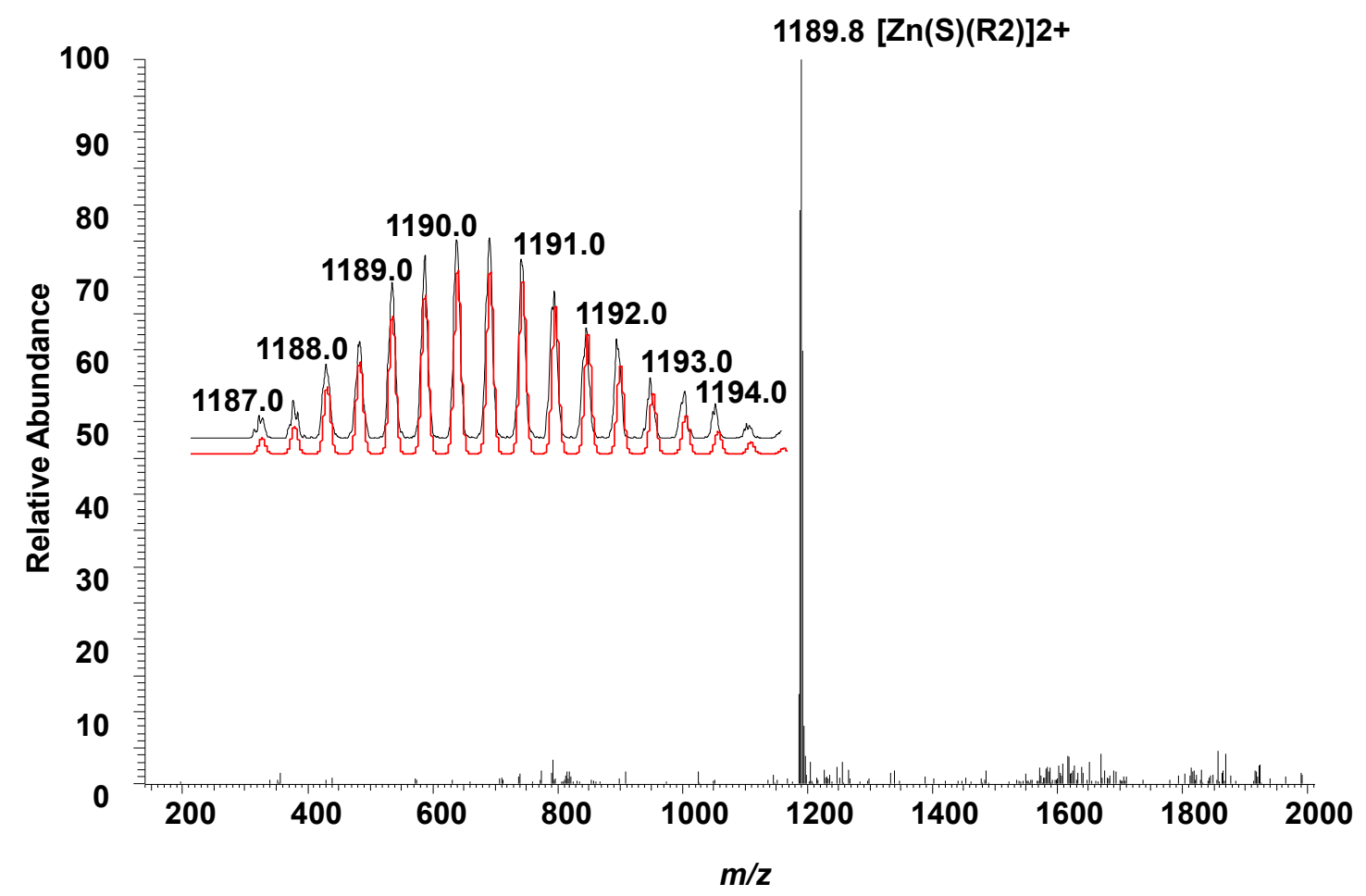

Figure S69. ESI-MS of nanorotor $[\mathrm{Zn}(\mathbf{S})(\mathbf{R 2})]^{+}$. 


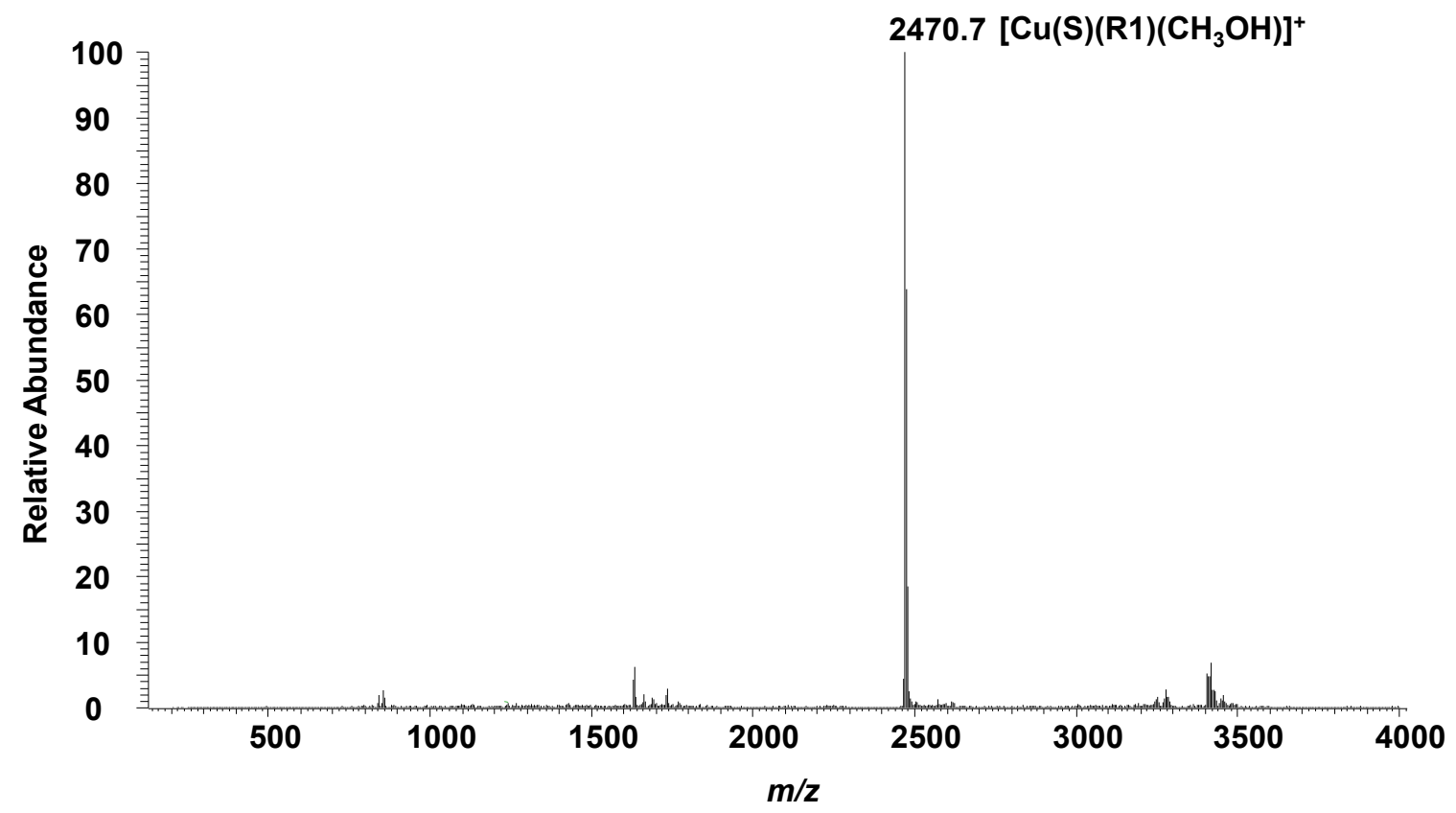

Figure S70. ESI-MS of nanorotor $\left[\mathrm{Cu}(\mathbf{S})(\mathbf{R 1})\left(\mathrm{CH}_{3} \mathrm{OH}\right)\right]^{+}$.

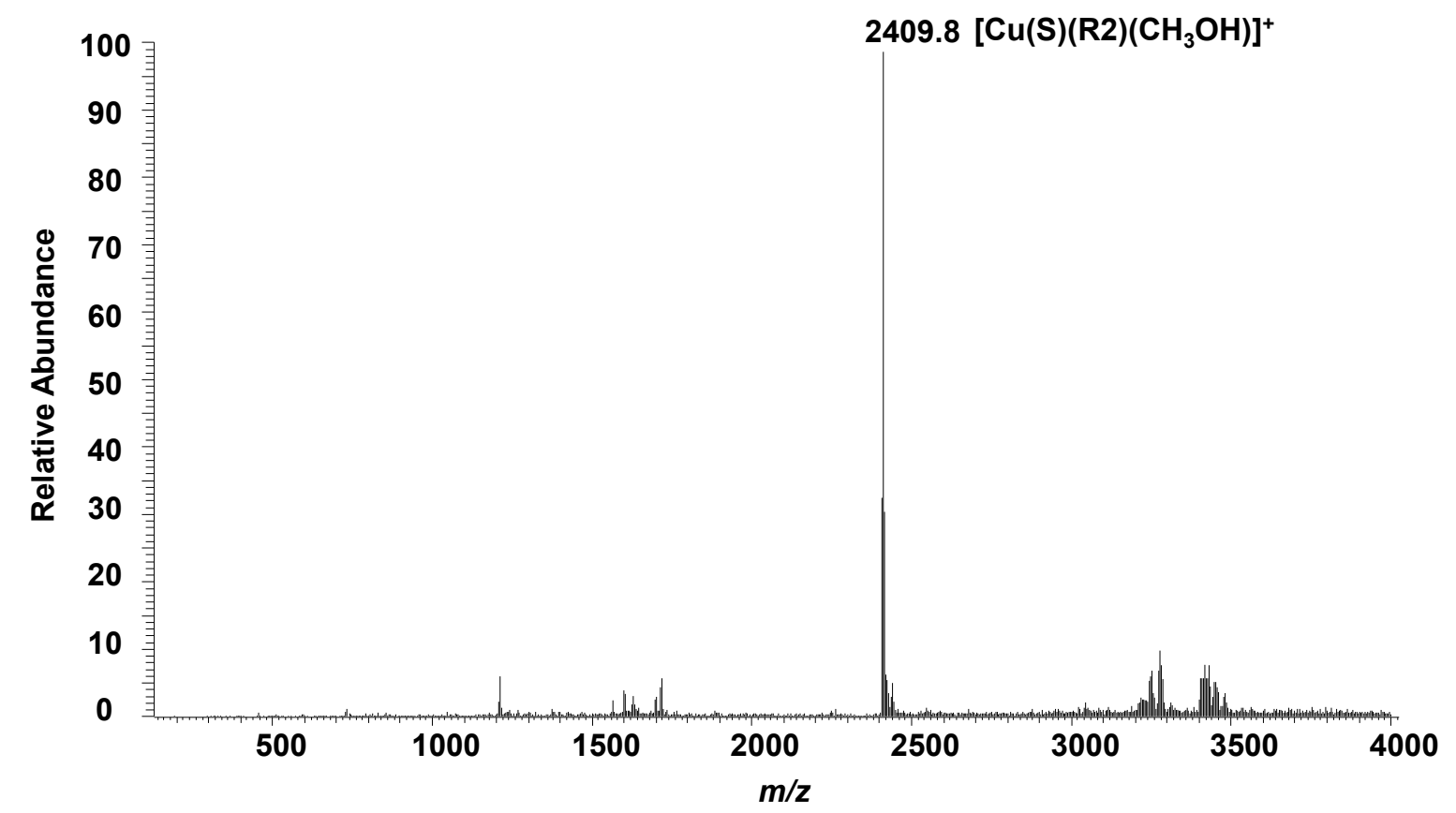

Figure S71. ESI-MS of nanorotor $\left[\mathrm{Cu}(\mathbf{S})(\mathbf{R 2})\left(\mathrm{CH}_{3} \mathrm{OH}\right)\right]^{+}$. 


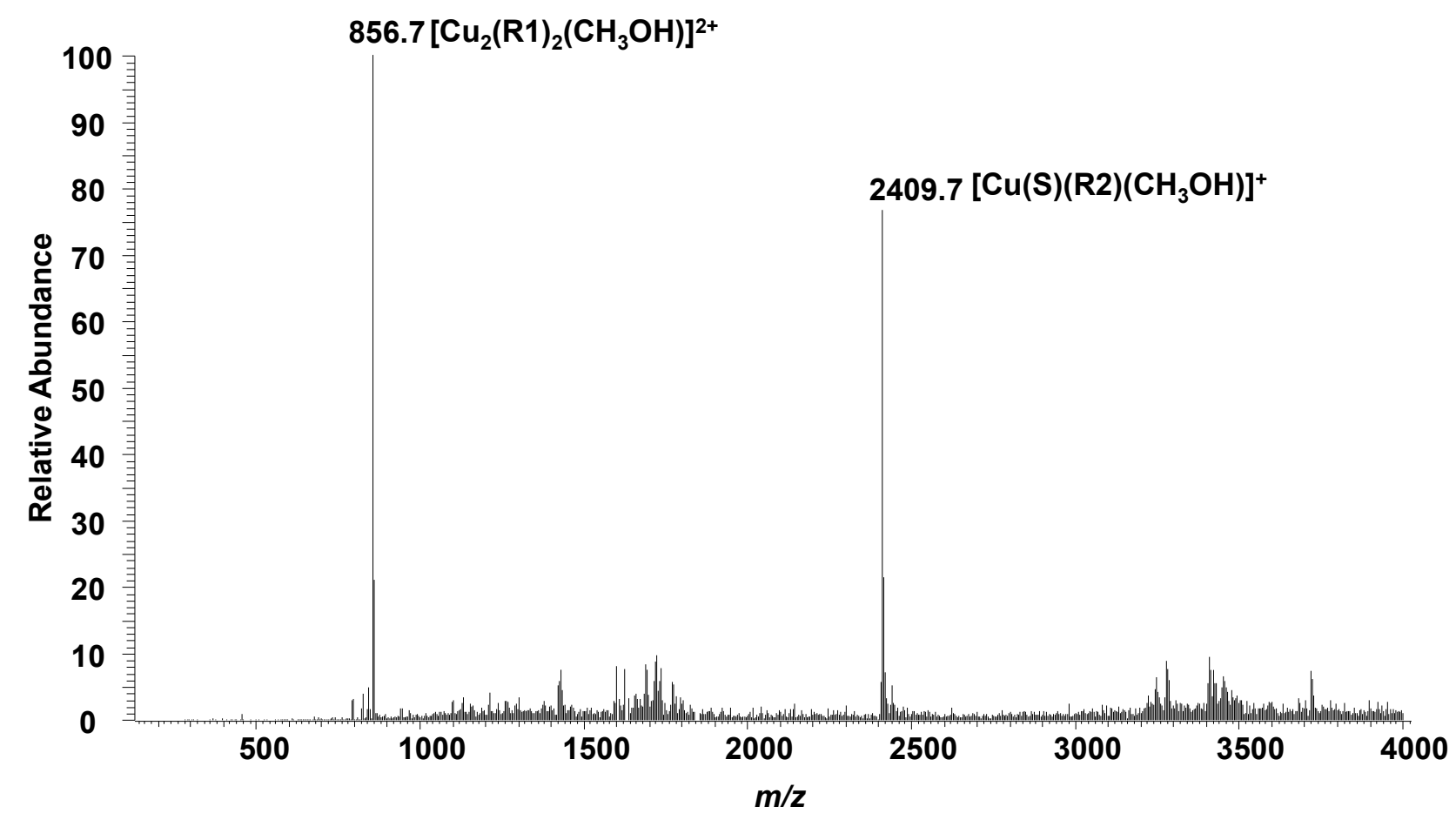

Figure S72. ESI-MS of NetState I, i.e. mixture of nanorotor $\left[\mathrm{Cu}(\mathbf{S})(\mathbf{R 2})\left(\mathrm{CH}_{3} \mathrm{OH}\right)\right]^{+}$and $\operatorname{dimer}\left[\mathrm{Cu}_{2}(\mathbf{R} \mathbf{1})_{2}\left(\mathrm{CH}_{3} \mathrm{OH}\right)\right]^{2+}$.

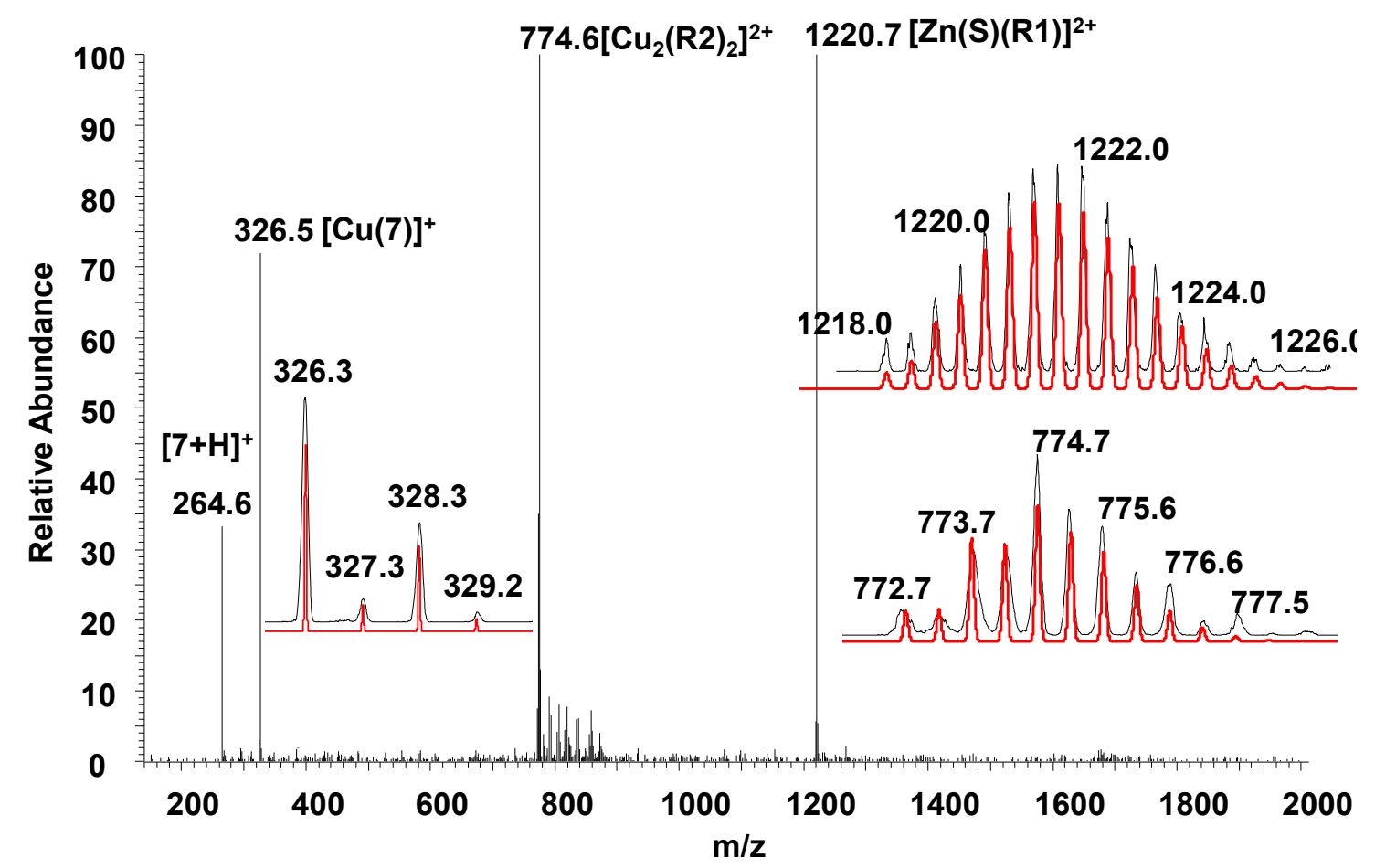

Figure S73. ESI-MS of NetState II, i.e. mixture of nanorotor $[\mathrm{Zn}(\mathbf{S})(\mathbf{R} 1)]^{2+}$ and dimer $\left[\mathrm{Cu}_{2}(\mathbf{R 2})_{2}\right]^{2+}$. 


\section{UV-vis data}

\section{Measurement of binding constants}

A UV-vis titration was used to measure binding constant of complex $\mathbf{7} \rightarrow \mathbf{4}$

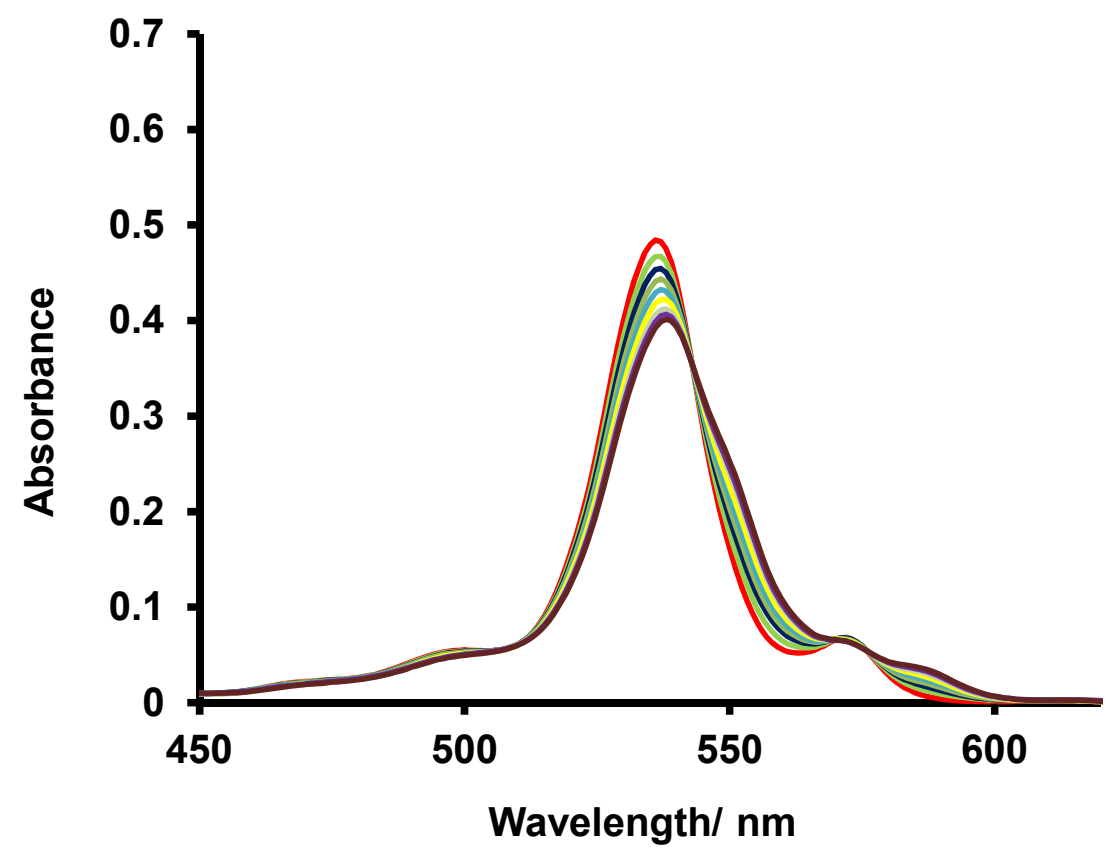

Figure S74. UV-vis titration of complex $4\left(1.2 \times 10^{-5} \mathrm{M}\right)$ vs. $7\left(1.1 \times 10^{-3} \mathrm{M}\right)$ in $\mathrm{CH}_{2} \mathrm{Cl}_{2}$ at $298 \mathrm{~K}$. Binding constant was determined to be $\log K=5.89 \pm 0.21$ using SPECFIT software

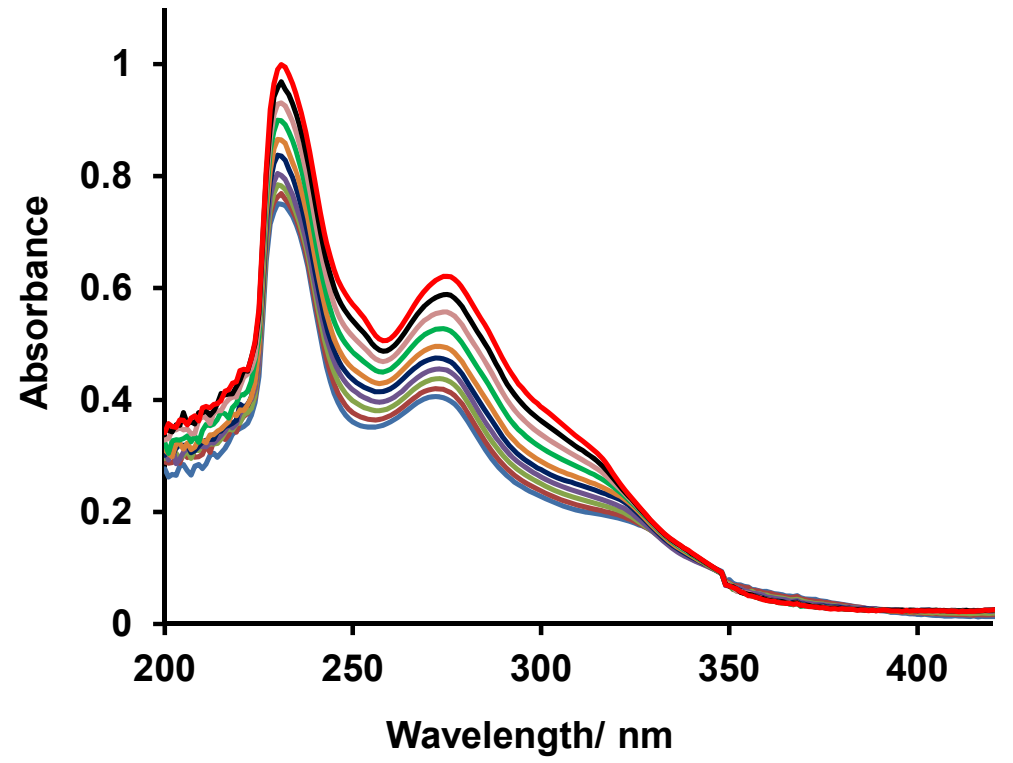

Figure S75. UV-vis titration of complex $[\mathrm{Cu}(2)]^{+}\left(4.2 \times 10^{-5} \mathrm{M}\right)$ vs. $6\left(2.2 \times 10^{-3} \mathrm{M}\right)$ in $\mathrm{CH}_{2} \mathrm{Cl}_{2}$ at $298 \mathrm{~K}$. Binding constant was determined to be $\log K=4.63 \pm 0.27$ using SPECFIT software. 


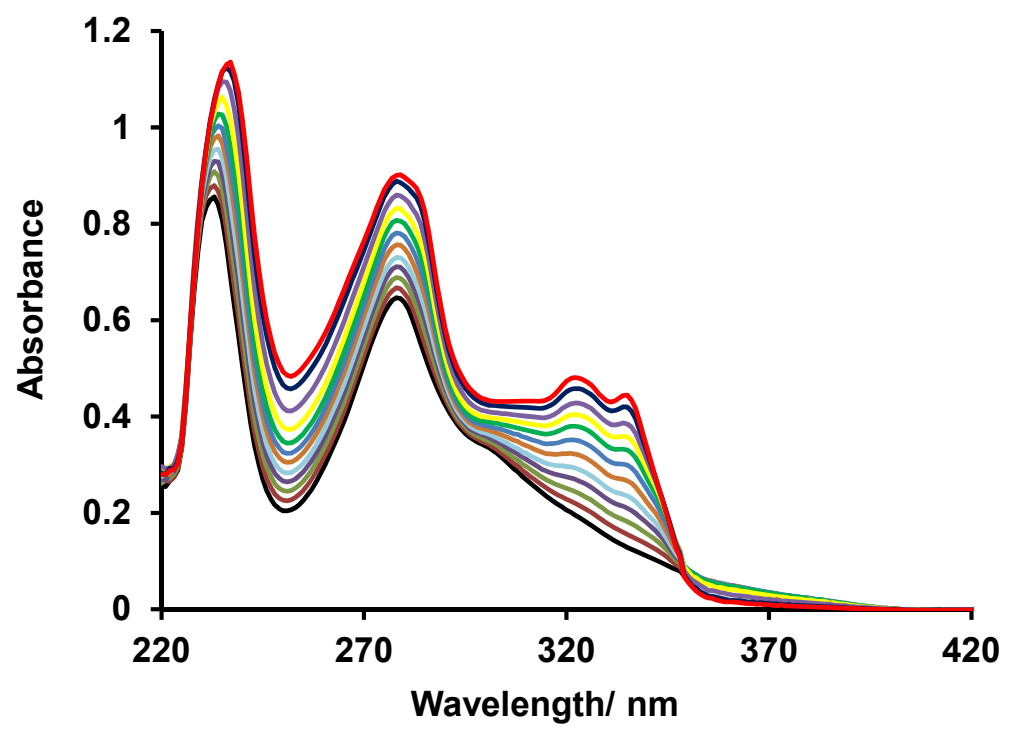

Figure S76. UV-vis titration of complex $[\mathrm{Zn}(\mathbf{5})]^{2+}\left(7.8 \times 10^{-5} \mathrm{M}\right)$ vs. $6\left(3.1 \times 10^{-3} \mathrm{M}\right)$ in $\mathrm{CH}_{2} \mathrm{Cl}_{2}: \mathrm{CH}_{3} \mathrm{CN}(98: 2)$ at 298 K. Binding constant was determined to be $\log K=5.26 \pm 0.18$ using SPECFIT software.

Table S1. Thermodynamic preference for NetState I

\begin{tabular}{|c|c|c|c|c|}
\hline \multicolumn{2}{|c|}{ NetState I } & \multicolumn{2}{|c|}{$\begin{array}{l}\text { Possible Alternate Combination I } \\
\text { (PAC I) }\end{array}$} & \multirow{2}{*}{$\begin{array}{c}\text { Overall thermodynamic } \\
\text { stabilization }(\Delta \log \beta) \\
\text { (NetState I-PAC I) }\end{array}$} \\
\hline $\begin{array}{c}\text { Isolated } \\
\text { complexation } \\
\text { units }\end{array}$ & $\begin{array}{c}\text { Thermodynamic } \\
\text { parameters } \\
(\log (\beta \text { or } K))\end{array}$ & $\begin{array}{c}\text { Isolated } \\
\text { complexation } \\
\text { units }\end{array}$ & $\begin{array}{c}\text { Thermodynamic } \\
\text { parameters } \\
(\log (\beta \text { or } K))\end{array}$ & \\
\hline$[\mathrm{Cu}(\mathbf{5})(\mathbf{6})]^{+}$ & $9.30^{6}$ & {$[\mathrm{Cu}(\mathbf{2})(\mathbf{6})]^{+}$} & $5.64^{8}+4.63^{\mathrm{a}}=10.27$ & $2^{b} \times(17.79-16.19)$ \\
\hline $3 \cdot 4$ & $4.31^{4}$ & $1 \cdot 4$ & $2.72^{4}$ & $=2 \times(1.6)=3.2$ \\
\hline$[\mathrm{Cu}(\mathbf{1})(\mathbf{2})]^{+}$ & $4.18^{7}$ & {$[\mathrm{Cu}(\mathbf{3})(\mathbf{5})]^{+}$} & $3.20^{9}$ & \\
\hline
\end{tabular}

a) see Figure S74. b) The factor 2 is dictated by the stoichiometric use of $\left[\mathrm{Cu}_{2}(\mathbf{R} 1, \mathbf{R 2})\right]^{2+}$.

Table S2. Thermodynamic preference for NetState II

\begin{tabular}{|c|c|c|c|c|}
\hline \multicolumn{2}{|c|}{ NetState II } & $\begin{array}{c}\text { Possible alternate combination II } \\
(\text { PAC II })\end{array}$ & \multirow{2}{*}{$\begin{array}{c}\text { Overall thermodynamic } \\
\text { stabilization }(\Delta \log \beta) \\
\text { (NetState II-PAC II) }\end{array}$} \\
$\begin{array}{c}\text { Isolated } \\
\text { complexation } \\
\text { units }\end{array}$ & $\begin{array}{c}\text { Thermodynamic } \\
\text { parameters } \\
(\log (\beta \text { or } K))\end{array}$ & $\begin{array}{c}\text { Isolated } \\
\text { complexation } \\
\text { units }\end{array}$ & $\begin{array}{c}\text { Thermodynamic } \\
\text { parameters } \\
(\log (\beta \text { or } K))\end{array}$ & \\
\cline { 1 - 3 }$[\mathrm{Zn}(\mathbf{2})(\mathbf{6})]^{2+}$ & $15.1^{10}$ & $\mathrm{Zn}(\mathbf{5})(\mathbf{6})]^{2+}$ & $4.58^{11}+5.26^{\mathrm{b}}=9.84$ & $2^{\mathrm{b}} \times(21.02-18.33)$ \\
$\mathbf{1 \cdot 4}$ & $2.72^{4}$ & $\mathbf{3 \cdot 4}$ & $4.31^{4}$ & $=2 \times(2.69)=5.38$ \\
{$[\mathrm{Cu}(\mathbf{3})(\mathbf{5})]^{+}$} & $3.20^{9}$ & {$[\mathrm{Cu}(\mathbf{1})(\mathbf{2})]^{+}$} & $4.18^{6}$ & \\
\hline
\end{tabular}

b) see Figure S75. b) The factor 2 is dictated by the stoichiometric use of $\left[\mathrm{Cu}_{2}(\mathbf{R} 1, \mathbf{R 2})\right]^{2+}$. 


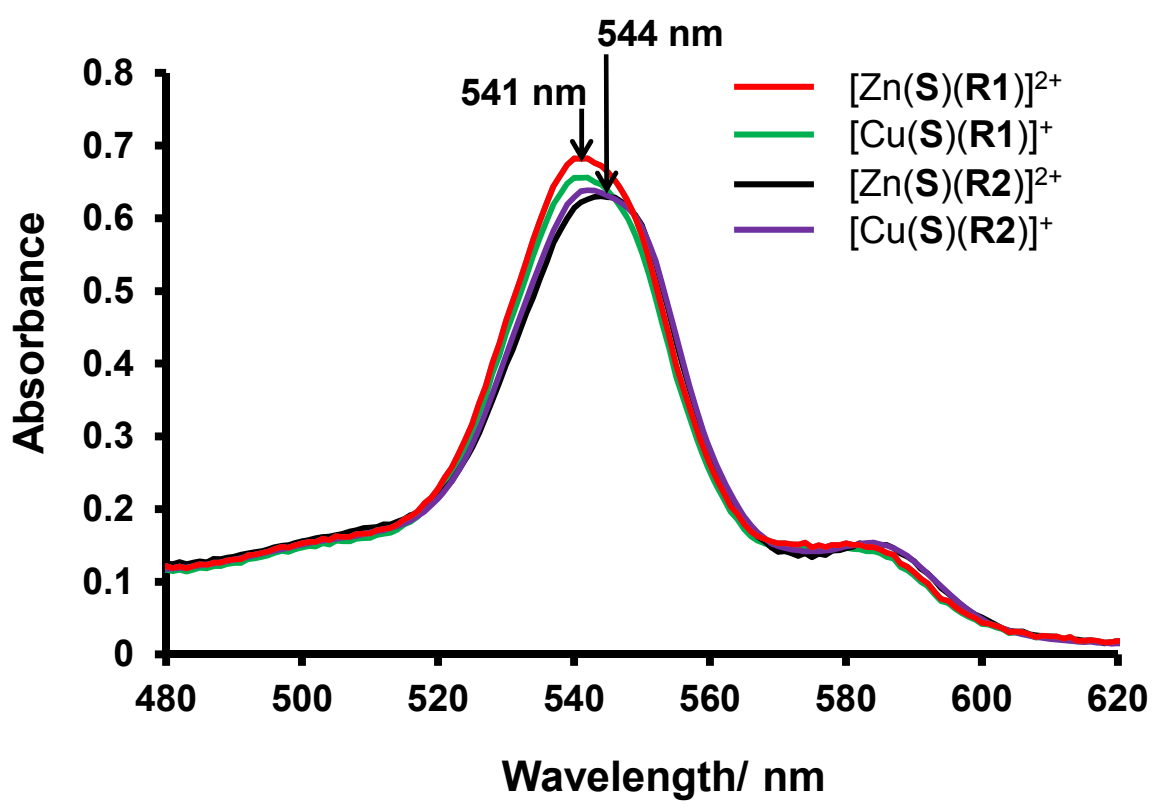

Figure S77. UV-vis spectra of nanorotor $[\mathrm{Zn}(\mathbf{S})(\mathbf{R} 1)]^{2+},[\mathrm{Cu}(\mathbf{S})(\mathbf{R} 1)]^{+},[\mathrm{Zn}(\mathbf{S})(\mathbf{R 2})]^{2+}$ and $[\mathrm{Cu}(\mathbf{S})(\mathbf{R 2})]^{+}\left(10^{-5} \mathrm{M}\right)$.

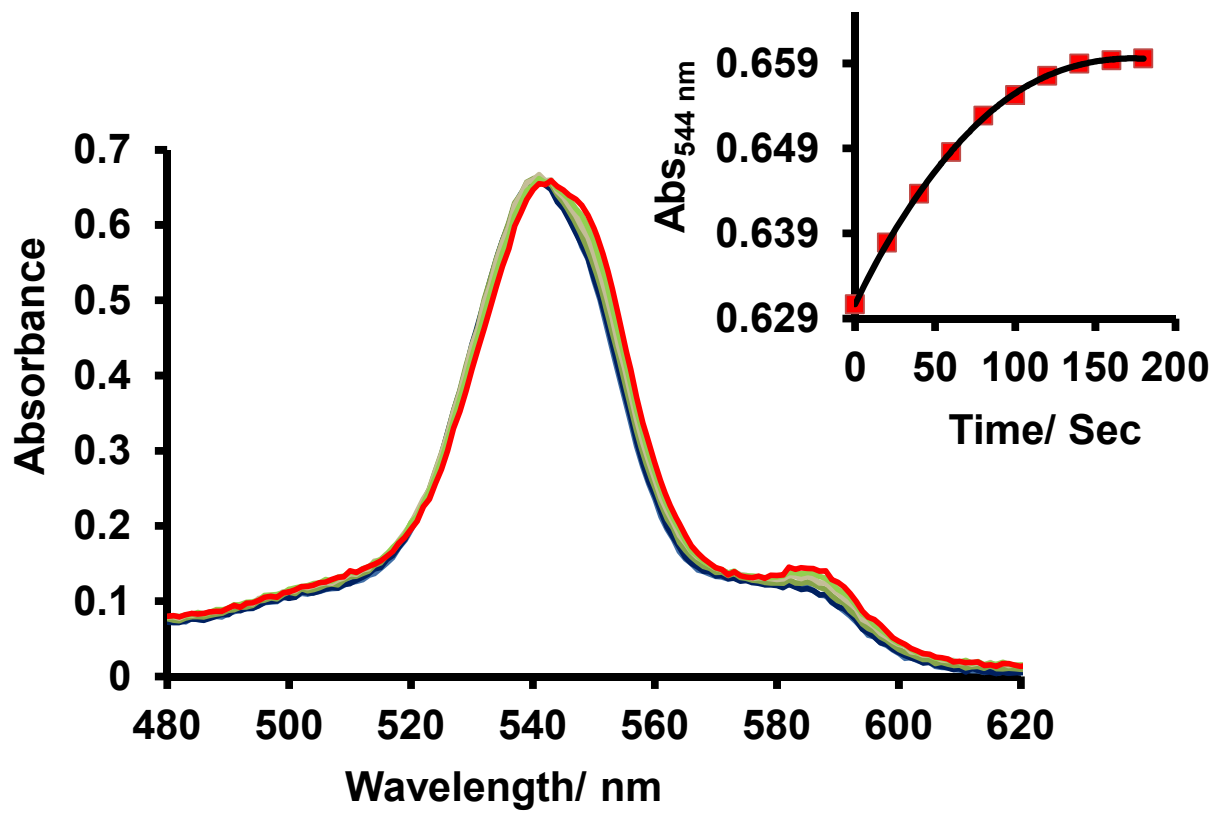

Figure S78. UV-vis spectra of the reaction between $[\mathrm{Cu}(\mathbf{S})(\mathbf{R 1})]^{+}$and $\left[\mathrm{Cu}_{2}(\mathbf{R 2})_{2}\right]^{2+}\left(1.2 \times 10^{-5} \mathrm{M}\right)$ at $298 \mathrm{~K} \mathrm{in} \mathrm{CH} 2 \mathrm{Cl} 2$ indicating formation of $[\mathrm{Cu}(\mathbf{S})(\mathbf{R 2})]^{+}$(and indirectly of $\left[\mathrm{Cu}_{2}(\mathbf{R} 1)_{2}\right]^{2+}$ ) with time. Inset: Change of absorbance at $\lambda=544$ nm with time. 


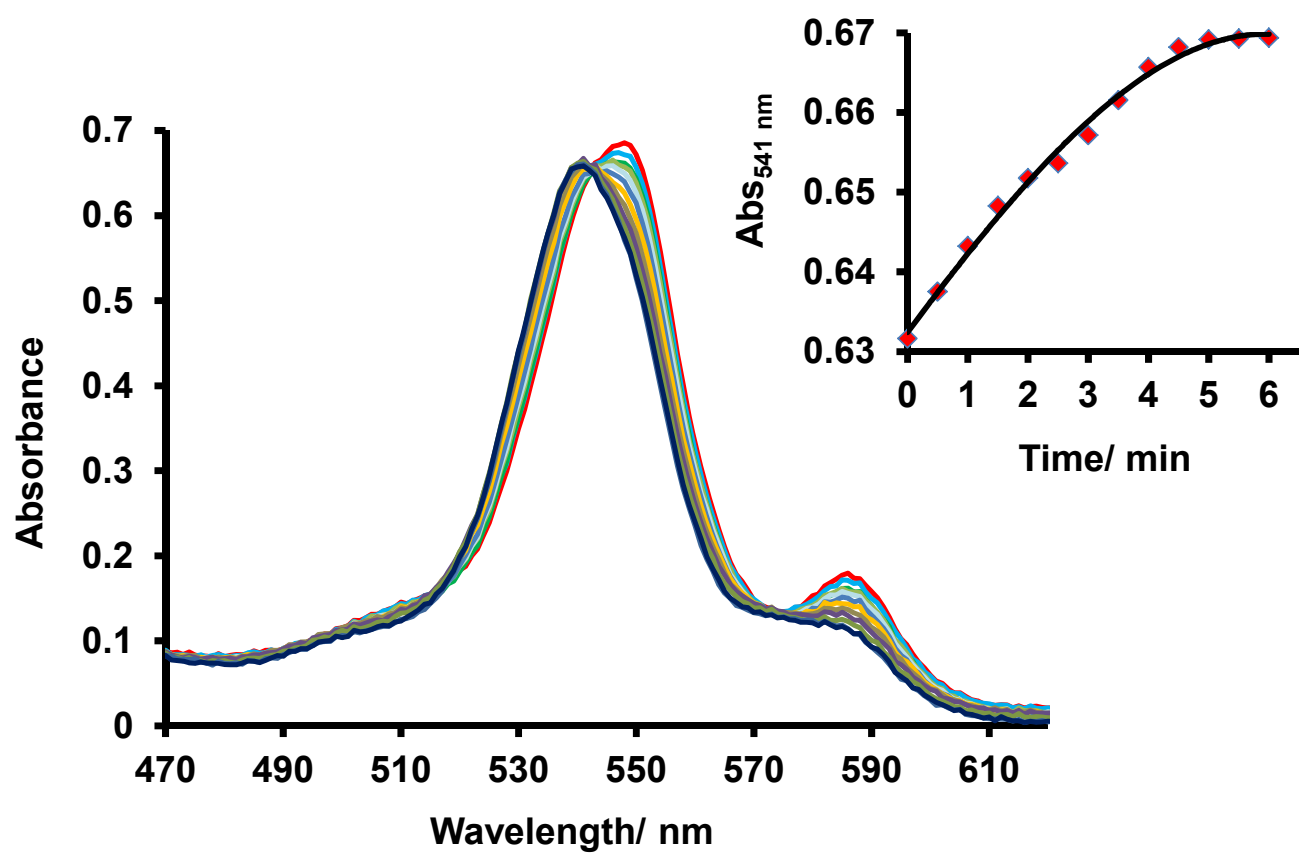

Figure S79. UV-vis spectra after addition of zinc(II) ions to the NetState I $\left([\mathrm{Cu}(\mathbf{S})(\mathbf{R 2})]^{+}\right.$and $\left.\left[\mathrm{Cu}_{2}(\mathbf{R} 1)_{2}\right]^{2+}\right)\left(1.2 \times 10^{-5}\right.$ M) at $298 \mathrm{~K}$ in $\mathrm{CH}_{2} \mathrm{Cl}_{2}$ indicating formation of $[\mathrm{Zn}(\mathbf{S})(\mathbf{R} 1)]^{2+}$ (and indirectly of NetState II) with time. Inset: Change of absorbance at $\lambda=541 \mathrm{~nm}$ with time.

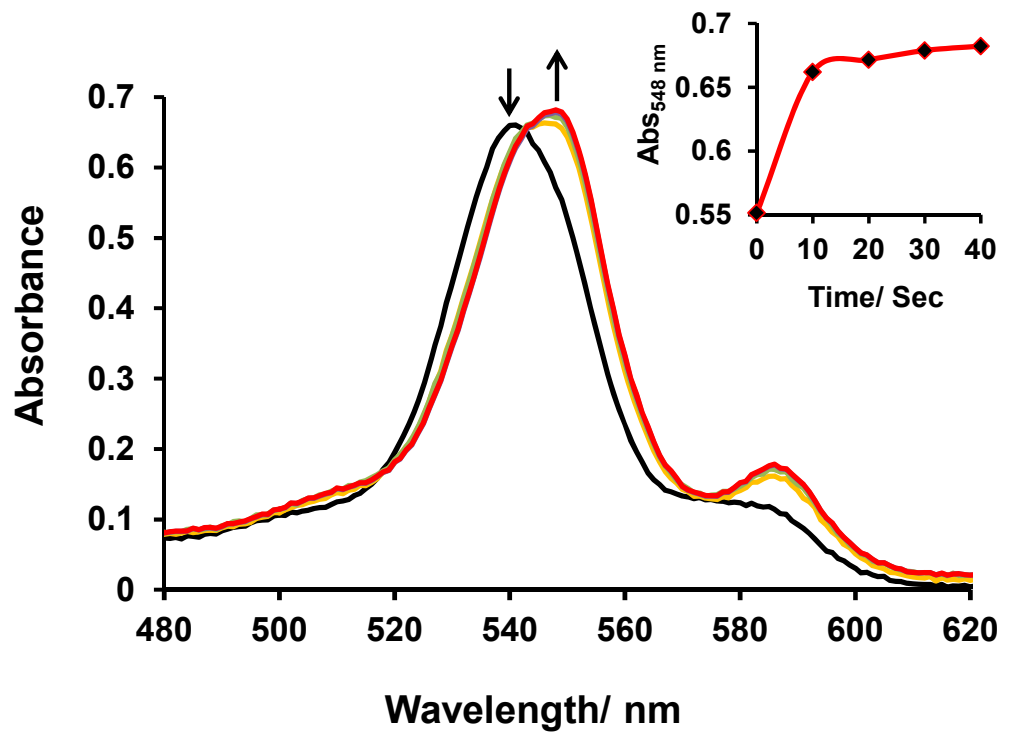

Figure S80. UV-vis spectra after addition of one equivalent of hexacyclen to NetState II $\left(=[\mathrm{Zn}(\mathbf{S})(\mathbf{R} 1)]^{2+}\right.$ and $\left.\left[\mathrm{Cu}_{2}(\mathbf{R 2})_{2}\right]^{2+}\right)\left(1.2 \times 10^{-5} \mathrm{M}\right)$ at $298 \mathrm{~K}$ in $\mathrm{CH}_{2} \mathrm{Cl}_{2}$ indicating formation of $[\mathrm{Cu}(\mathbf{S})(\mathbf{R 2})]^{+}$(and indirectly of NetState I) with time. Inset: Change of absorbance at $\lambda=548 \mathrm{~nm}$ with time. 


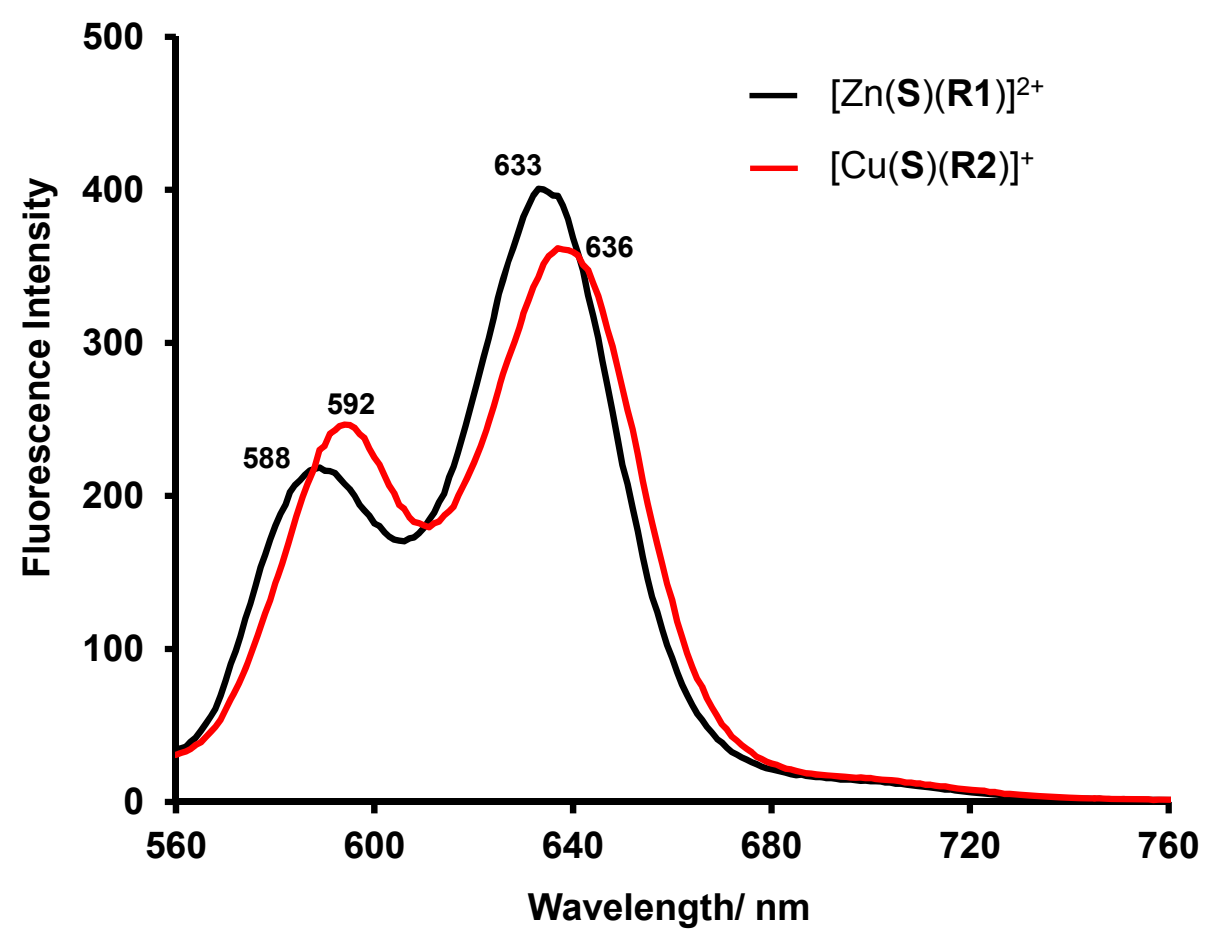

Figure S81. Fluorescence spectra of $[\mathrm{Zn}(\mathbf{S})(\mathbf{R} 1)]^{2+}$ and $[\mathrm{Cu}(\mathbf{S})(\mathbf{R 2})]^{+}\left(2.3 \times 10^{-5} \mathrm{M}\right.$ in $\mathrm{CD}_{2} \mathrm{Cl}_{2}: \mathrm{CD}_{3} \mathrm{CN}(5: 1)$, excitation wavelength: $540 \mathrm{~nm}$ ).

\section{References}

(1) Goswami, A.; Paul, I.; Schmittel, M. Chem. Commun. 2017, 53, 5186-5189.

(2) Gaikwad, S.; Goswami, A.; De, S.; Schmittel, M. Angew. Chem. Int. Ed. 2016, 55, 10512 10517.

(3) Walter, K. A.; Kim, Y. J.; Hupp, J. T. Inorg. Chem. 2002, 41, 2909.

(4) Paul, I.; Goswami, A.; Mittal, N.; Schmittel, M. Angew. Chem., Int. Ed. 2018, 57, 354-358.

(59 Schmittel, M.; Michel, C.; Wiegrefe, A.; Kalsani, V. Synthesis, 2001, 1561 - 1567.

(6) Schmittel, M.; Kalsani, V.; Kishore, R. S. K.; Cölfen, H.; Bats, J. W. J. Am. Chem. Soc. 2005, $127,11544-11545$.

(7) Ghosh, A.; Paul, I.; Saha, S.; Paululat, T.; Schmittel M. Org. Lett. 2018, 20, 7973-7976.

(8) Paul, I.; Samanta, D.; Gaikwad, S.; Schmittel, M. Beilstein J. Org. Chem. 2019, 15, 13711378.

(9) Samanta, S. K.; Schmittel, M. J. Am. Chem. Soc. 2013, 135, 18794-18797.

(10) Samanta, D.; Paul, I.; Schmittel, M. Chem. Commun. 2017, 53, 9709-9712.

(11) Özer, M. S.; Paul, I.; Goswami, A.; Schmittel, M. Dalton Trans., 2019,48, 9043-9047. 